SLAC-R-972

\title{
Search for Large Extra Dimensions Based on Observations of Neutron Stars with the Fermi-LAT
}

\author{
By Bijan Berenji
}


SEARCH FOR LARGE EXTRA DIMENSIONS BASED ON OBSERVATIONS OF NEUTRON STARS WITH THE FERMI-LAT

\title{
A DISSERTATION
}

SUBMITTED TO THE DEPARTMENT OF APPLIED PHYSICS AND THE COMMITTEE ON GRADUATE STUDIES OF STANFORD UNIVERSITY

IN PARTIAL FULFILLMENT OF THE REQUIREMENTS

FOR THE DEGREE OF DOCTOR OF PHILOSOPHY

\author{
Bijan Berenji \\ SLAC-R-972 \\ September 2011
}

PREPARED FOR THE DEPARTMENT OF ENERGY UNDER CONTRACT DE-AC03-765F00515 
() 2011 by Bijan Berenji. All Rights Reserved.

Re-distributed by Stanford University under license with the author.

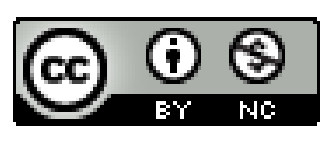

This work is licensed under a Creative Commons AttributionNoncommercial 3.0 United States License.

http://creativecommons.org/licenses/by-nc/3.0/us/

This dissertation is online at: http://purl.stanford.edu/sj534tb9150 
I certify that I have read this dissertation and that, in my opinion, it is fully adequate in scope and quality as a dissertation for the degree of Doctor of Philosophy.

Elliott Bloom, Primary Adviser

I certify that I have read this dissertation and that, in my opinion, it is fully adequate in scope and quality as a dissertation for the degree of Doctor of Philosophy.

\section{Aharon Kapitulnik, Co-Adviser}

I certify that I have read this dissertation and that, in my opinion, it is fully adequate in scope and quality as a dissertation for the degree of Doctor of Philosophy.

Peter Graham

Approved for the Stanford University Committee on Graduate Studies.

Patricia J. Gumport, Vice Provost Graduate Education

This signature page was generated electronically upon submission of this dissertation in electronic format. An original signed hard copy of the signature page is on file in University Archives. 
Published by

Stanford Linear Accelerator Center

Technical Publications MS 68

2575 Sand Hill Road

Menlo Park, CA 94025

This document and the material and data contained herein was developed under the sponsorship of the United States Government. Neither the United States nor the Department of Energy, nor the Leland Stanford Junior University, nor their employees, makes any warranty, express or implied, or assumes any liability or responsibility for accuracy, completeness or usefulness of any information, apparatus, product or process disclosed, or represents that its use will not infringe privately owned rights. Mention of any product, its manufacturer, or suppliers shall not, nor it is intended to, imply approval, disapproval, or fitness for any particular use. A royalty-free, non-exclusive right to use and disseminate same for any purpose whatsoever, is expressly reserved to the United States and the University. 


\section{Abstract}

According to the Large Extra Dimensions (LED) model of Arkani-Hamed, Dimopoulos, and Dvali (ADD), in addition to the $(3+1)$ observed space-time dimensions, there exist $\mathrm{n}$ gravity-only spatial dimensions. Due to the presence of the additional dimensions, the Planck scale of gravity should be brought down from $10^{16} \mathrm{TeV}$ to the $\mathrm{TeV}$ scale, near the electroweak scale, and thus solve the hierarchy problem. Based on the ADD theory, Kaluza-Klein (KK) gravitons, having masses of the order $100 \mathrm{MeV}$ and lifetimes of the order of billions of years, are expected to be produced within supernova cores by nucleon-nucleon gravi-bremsstrahlung in the LED model. Once produced, they are predicted to be trapped by the gravitational potential of subsequently formed neutron stars (NS), and their decay is predicted to contribute to a measurable gamma-ray flux from NS.

In this dissertation, refinements to past theoretical models are made, including modifications for the expected spectral energy distribution based on orbital motion of the KK gravitons, and magnetic fields and age. $n=2,3, \ldots, 7$ extra dimensions are considered. A sample of 6 gamma-ray faint NS sources not reported in the first Fermi gamma-ray source catalog that are good candidates are selected for this analysis, based on age, surface magnetic field, distance, and galactic latitude. Based on 11 months of data from Fermi-LAT, 95\% CL upper limits on the size of extra dimensions $R$ from each source are obtained, as well as 95\% CL lower limits on the $(n+4)$ dimensional Planck scale $M_{D}$. In addition, the limits from all of the analyzed NSs have been combined statistically using two likelihood-based methods. The results indicate more stringent limits on LED than quoted previously from individual neutron star sources in gamma-rays. In addition, the results are more stringent than current 
collider limits, from the LHC, for $n<4$, and comparable for $n=4$. If the Planck scale is around a $\mathrm{TeV}$, then for $n=2,3$, the compactification topology must be more complicated than a torus. For $n>3$, the toroidal topology is still allowed. 


\section{Preface}

This dissertation is dedicated to physicists, past and present, theoretical and experimental, who have tried to unify gravity with the the other fundamental forces. Hopefully, this work will help the experimental effort in this respect. 


\section{Acknowledgments}

I would like to thank my Mom and Dad for supporting me through the years of graduate school at Stanford. I would like to thank my sister Manijeh for being supportive. My brother Shahin, you always had entertaining stories and anecdotes, thanks for keeping me in positive spirits. I would like to thank my graduate student colleagues Yvonne Edmonds, Ping Wang, and Alex Drlica-Wagner for helpful discussions on Fermi-LAT analysis and camaraderie. I would like to thank the other Fermi-LAT graduate students for their friendship and help. I would like to thank the postdocs who gave me advice along the way. In particular, I would like to thank Dr. Simona Murgia, for being supportive of my research in general, and for promoting my presentation at Aspen Winter Conference in February 2011. I would like to recognize the dedication of all of the members of the Fermi-LAT collaboration, without whom this work couldn't proceed. I am in gratitude to my adviser, Elliott Bloom, who contributed immensely to my progress as a experimental particle astrophysicist, and encouraged me to be a critical thinker in physics. I would like to thank all the physics professors that I have encountered, who have nurtured my skills in physics

Regarding institutions, I would like to thank the Department of Energy for providing the source of funding for my work. I would like to thank the people of SLAC National Accelerator Laboratory for making it an inviting place to do research for Stanford University students. I would also like to express my appreciation for the faculty and staff of the Applied Physics Department at Stanford University. 


\section{Acronyms}

ACD Anti-Coincidence Detector

ADD Arkani-Hamed, Dimopoulos, Dvali

CL Confidence Level

CMS Compact Muon Solenoid

CT Classification Tree

IRF Instrument Response Function

KK Kaluza-Klein

NS Neutron Star

LAT Large Area Telescope

LED Large Extra Dimensions

LHC Large Hadron Collider

PSF Point Spread Function

PSR Pulsar

ROI Region of Interest

RS Randall-Sundrum

SED Spectral Energy Distribution 


\section{SN Supernova}

UL Upper Limit 


\section{Contents}

Abstract v v

Preface vii

Acknowledgments ix

Acronvms

1 Introduction 1

1.1 Classical and Relativistic Gravity . . . . . . . . . . . . . . 1

1.2 Bevond Classical and Relativistic Gravity . . . . . . . . . . . . 2

1.3 Shortcomings of the Standard Model $\ldots \ldots \ldots \ldots$

1.4 Model of Large Extra Dimensions . . . . . . . . . . . . . . . . 4

$1.5 \quad$ Graviton Emission $\ldots \ldots \ldots \ldots \ldots$

1.6 Previous Experimental Constraints . . . . . . . . . . . . . . 10

1.6.1 Tabletop Experiments of the Inverse Square Force Law . . . . 10

1.6 .2 Collider Constraints . . . . . . . . . . . . . . . 11

1.6.3 Astrophysical Constraints . . . . . . . . . . . . . . . . 13

1.7 Other Extra Dimension Scenarios . . . . . . . . . . . . . . . . 16

1.7 .1 Randall-Sundrum Model . . . . . . . . . . . . . . . . . 16

1.7 .2 LED Extra Dimensions with Warping . . . . . . . . . . 16

1.8 Universal Extra Dimensions . . . . . . . . . . . . . . . . . . . 17 
2 Experimental Considerations $\quad 19$

2.1 The Fermi-LAT Instrument . . . . . . . . . . . . . . . . . . . . . . 19

2.1 Tracker ............................. 19

2.1 .2 Calorimeter . . . . . . . . . . . . . . . . . . . . . 22

2.1.3 Anti-Coincidence Detecton ............... 24

2.1.4 LAT Data Acquisition and Electronics Modules . . . . . . . 25

2.1 .5 Event Reconstruction in the LAT . . . . . . . . . . . . . 25

2.2 LAT Performance . . . . . . . . . . . . . . . . . . . . . . . 28

2.2 .1 Instrument Response Functions . . . . . . . . . . . . . . 28

2.2.2 Point Spread Function of the LAT . . . . . . . . . . . . . . . . 29

2.2 .3 Energv Resolution . . . . . . . . . . . . . . . . . . 30

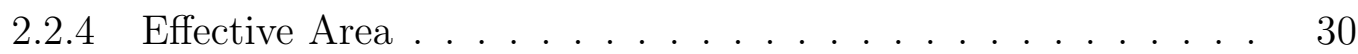

2.2.5 Comparison with EGRET . . . . . . . . . . . . . . . . 32

3 Obtaining the Differential Flux 33

3.1 Emission of KK Gravitons by $N N$ Bremsstrahlung . . . . . . . . . . 34

3.1.1 KK Gravitons in in the Gravitational Potential of a proto-NS 38

3.2 Theoretical Model . . . . . . . . . . . . . . . . . . . . . 39

3.2.1 Determining the differential flux by Monte Carlo Simulation . 41

3.3 Differential Fluxes for the Various Sources . . . . . . . . . . . . . . . 53

4 Neutron Star Properties $\quad 59$

4.1 Birth of Neutron Stars in Supernova Core Collapse . . . . . . . . . . 59

4.2 Properties of Neutron Stars . . . . . . . . . . . . . . . . . . . . . . . 62

4.3 Choosing Neutron Stars for Analysis . . . . . . . . . . . . . . . . . . 64

4.4 RXJ1856.35-3754 . . . . . . . . . . . . . . . . . . 66

$4.5 \quad \mathrm{PSR}$ J0108-1431 . . . . . . . . . . . . . . . . . . . . . . . . . 68

4.6 PSR J0630-2834 . . . . . . . . . . . . . . . . . . . . . . . . . . . 68

4.7 PSR J0953+0755 . . . . . . . . . . . . . . . . . . . . . . . 69

4.8 PSR J1136+1551 . . . . . . . . . . . . . . . . . . . . . . . . . . 69

$4.9 \mathrm{PSR} \mathrm{J0826+2637} \ldots \ldots \ldots$ 
5 Data Analysis Methods $\quad 71$

5.1 Fermi Science Tools . . . . . . . . . . . . . . . . . . . . . . . 71

5.1.1 Performing Selections on Data and Making Good Time Intervals 72

5.1 .2 Generating Livetime Cubes . . . . . . . . . . . . . . 72

5.1 .3 Generating Counts Maps . . . . . . . . . . . . . . . 73

5.1 .4 Generating Exposure Maps ... . . . . . . . . . . . 74

5.2 Model Fitting . . . . . . . . . . . . . . . . . . . . . . . 75

5.2 .1 The Log-Likelihood Function . . . . . . . . . . . . . . . . . . 75

5.2 .2 Test Statistid . . . . . . . . . . . . . . . . . . . 76

5.2 .3 Fitting with gtlike ................ 76

5.2.4 Likelihood based fitting with pvthon interface . . . . . . . . 77

5.2 .5 Using Fitted Model . . . . . . . . . . . . . . . . . . . . . 77

5.2 .6 Implementing a Custom Model for Fitting . . . . . . . . . . . 77

5.3 Computing Upper Limits with MINOS Errors . . . . . . . . . . . . . 78

5.4 Combining Limits . . . . . . . . . . . . . . . . . . . . . . . . . . . . . 79

5.4 .1 Using MINOS Errors . . . . . . . . . . . . . . . . . . . . . 79

5.5 Computing Limits with Profile Likelihood . . . . . . . . . . . . . . . 80

6 Modeling ROIs and Setting Limits on LED 81

6.1 Data Sample in Analysis . . . . . . . . . . . . . . . . . . . . 81

6.2 Modeling ROIs . . . . . . . . . . . . . . . . . . . . . . . . 81

6.3 Limits Results . . . . . . . . . . . . . . . . . . . . . . . 87

6.3 .1 Individual Limits . . . . . . . . . . . . . . . . . . . 87

6.4 Interpretation . . . . . . . . . . . . . . . . . . . . . . . . . 91

7 Validation Studies $\quad 93$

7.1 Verifving Upper Limits Using Different Technique . . . . . . . . . . . . 93

7.2 Svstematic Errors from 1FGL Parameters and Diffuse Parameters . . 94

7.3 Simulations of sources within the ROI for RXJ1856-3754 . . . . . . 98

7.4 Using an Updated IRF . . . . . . . . . . . . . . . . . . . . . . . . . . . . 99 
8 Conclusions 101

8.1 Interpretation . . . . . . . . . . . . . . . . . 101

8.2 Generalization of LED Bounds . . . . . . . . . . . . . . . . . . . . 101

8.2.1 Weakly-warped Extra Dimensions . . . . . . . . . . . . . . 101

8.2.2 Axion-Like Particles _. . . . . . . . . . . . . . . . . . 102

8.3 Possible Future Improvements . . . . . . . . . . . . . . . . . . . . . . 102

8.3.1 Theoretical Improvements _ . . . . . . . . . . . . . . . . . . . 102

8.3.2 Instrumental Improvements . . . . . . . . . . . . . . . . . . . 105

8.3.3 Data Analvsis Improvements . . . . . . . . . . . . . . 106

8.4 Considering a Population of NS in the Galactic Center . . . . . . . . 106

8.5 Constraining LED with Diffuse Gamma-Rav Emission . . . . . . . . . 107

$\begin{array}{ll}\text { Appendices } & 108\end{array}$

A Fitted Differential Flux Distributions for Each NS Source 111

B Sources in the Vicinity of Neutron Stars $\quad 119$

C Fitting Catalog Defined ROIs $\quad 125$

C.0.1 Introduction . . . . . . . . . . . . . . . . . . . . . . 125

C.0.2 Fitting ROIs . . . . . . . . . . . . . . . . . . 126

C.0.3 Fitting ROI Results . . . . . . . . . . . . . . . . . . . 126

D Studies with MC of Low Energy Gamma Rays 129

D.1 Introduction . . . . . . . . . . . . . . . . . . . . . 129

D.2 Comparison of Energy Variable with True Energy . . . . . . . . . . . 131

D.3 Energy Resolution Studies . . . . . . . . . . . . . . . . . . . 132

D.4 Calculation of Mean Energy and Energy Resolution . . . . . . . . . . 132

D.5 Conclusions . . . . . . . . . . . . . . . . . . . . . 135

$\begin{array}{lr}\text { References } & 137\end{array}$ 


\section{List of Tables}

2.1 Comparison of Fermi-LAT and EGRET performance. . . . . . . . . 32

$3.1 n$-dependent constants for trapped KK gravitons. . . . . . . . . . . 39

3.2 Mean radial distance from NS center. for different $n \ldots \ldots \ldots$

3.3 Table of values of the attenuation parameter n . . . . . . . . 54

4.1 Astrophvsical Properties of 6 NS sources Analvzed . . . . . . . . . 65

6.1 Table of diffuse parameters for ROIs corresponding to each source. . . 83

6.2 Table of flux upper limits . . . . . . . . . . . . . . . . . . 84

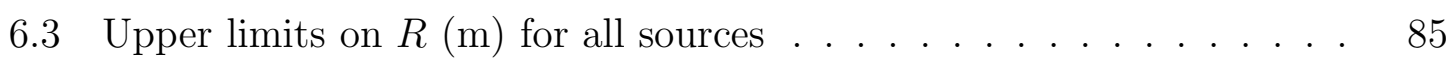

6.4 Lower limits on $M_{D}(\mathrm{TeV}) \ldots \ldots \ldots \ldots \ldots \ldots$

6.5 Comparison of upper limits on size of extra dimensions . . . . . . 87

6.6 Comparison of $95 \%$ CL lower limits on $M_{D}(\mathrm{TeV}) \ldots \ldots \ldots$

7.1 Upper limits on flux for RXJ1856-3754 from UpperLimits . . . . . . 93

7.2 Parameters of flux distributions for J1856-3754. from MC studv. . . . 94

7.3 97.5\% CL flux upper limit values from atobssim studv of J1856-3754. 98

7.4 Comparison of upper limits for RXJ1856-3754 in two Pass 6 IRFs . . 99

8.1 Table of values of $k_{n}$. evaluated at different temperatures. . . . . 103

8.2 95\% CL upper limits on $R(\mathrm{~m})$. J1856-3754, evaluated for different $T 104$

B.1 Table of 1FGL sources $14^{\circ}$ of RXJ1856-3754 . . . . . . . . . . 120

B.2 Table of 1FGL sources within $14^{\circ}$ of PSR J0108-1431. . . . . . . . 121

B.3 Table of 1FGL sources within $14^{\circ}$ of PSR J0953+0755. . . . . . . . 121 
B.4 Table of 1FGL sources within $14^{\circ}$ of PSR J0630-2834 . . . . . . . . . 122

B.5 Table of 1FGL sources within $14^{\circ}$ of PSR J1136+1551. . . . . . . 123

B.6 Table of 1FGL sources within $14^{\circ}$ of PSR J0826-2637. . . . . . . . . 124

C.1 Comparison of mv fit values and 1FGL fit values for a given ROI . . . 127 


\section{List of Figures}

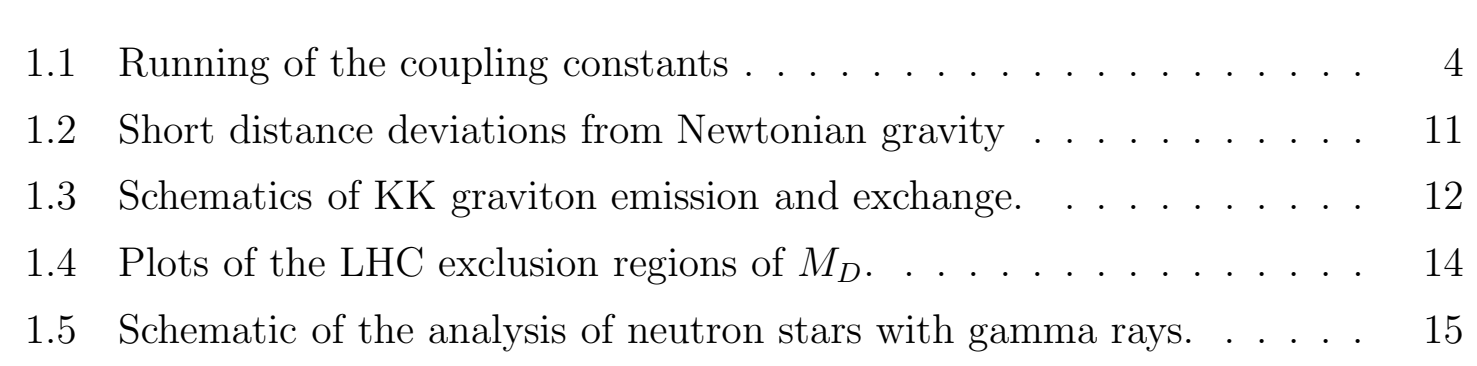

2.1 A schematic of the LAT . . . . . . . . . . . . . 20

2.2 A single tracker trav . . . . . . . . . . . . . . . . . 21

2.3 Above view of the $4 \times 4$ tracker arrav before integration with the ACD. 22

2.4 Schematic of tracker lavers . . . . . . . . . . . . . . 23

2.5 Schematic of calorimeter . . . . . . . . . . . . . . . . 24

2.6 Schematic of ACD . . . . . . . . . . . . . . . 25

2.7 Schematic view of electronics modules for the LAT. . . . . . . . . . 26

2.8 Schematic diagrams of a gamma-rav event in the LAT . . . . . . . . . 27

2.9 PSF plots for the LAT . . . . . . . . . . . . . . . . 30

2.10 Energy resolution curve for the P6_V3_DIFFUSE IRF . . . . . . . . . 31

2.11 Effective area plots . . . . . . . . . . . . . . . . . . . . . . . . . . 31

3.1 The Fevnman diagram for KK graviton emission . . . . . . . . . . . . 34

3.2 Distributions of KK graviton masses _ . . . . . . . . . . . 42

3.3 Radial probability density functions, $P(r: \mu)$. for 2 different values of $\mu$. 45

3.4 KK graviton trajectories . . . . . . . . . . . . . . . . . . 46

3.5 Radial distribution of decav vertices. $n=2$ case averaged over $\mu$. . 47

$3.6 \gamma$-rav energy distributions from graviton decav for $n=2,5.7 \ldots$. . 48 
3.7 Coordinate svstem for $G_{K K}$ decav, as described in step (6) . . . . . . 50

3.8 Plot of the reduced attenuation coefficient. $\alpha_{1}(\chi)$. . . . . . . . . . 52

3.9 Differential fluxes for $n=2 \quad \ldots \ldots \ldots \ldots$. . . . . . . . . . 55

3.10 Differential fluxes for $n=3$. . . . . . . . . . . . . 55

3.11 Differential fluxes for $n=4$. . . . . . . . . . . . . . 56

3.12 Differential fluxes for $n=5$. . . . . . . . . . . . . 56

3.13 Differential fluxes for $n=6$. . . . . . . . . . . . . 57

3.14 Differential fluxes for $n=7$. . . . . . . . . . . . . 57

4.1 Contours for $\rho$ vs. $T$ for different $Y_{D} \ldots \ldots$. . . . . . . . . 60

4.2 Schematic of the core-collapse event . . . . . . . . . . . . . . 61

4.31 vr Fermi skv intensity map $\left(\mathrm{cm}^{-2} \mathrm{~s}^{-1}\right)$. plotted in galactic coordinates. 65

4.4 A schematic of a pulsar . . . . . . . . . . . . . . . . . 67

6.1 Counts residuals plot and flux plot. RXJ1856-3754 . . . . . . . . . . 83

6.2 Counts map and model map. RXJ1856-3754 . . . . . . . . . . . . . . 84

6.3 Counts residual map for RXJ1856-3754 . . . . . . . . . . . . . . . . . 84

6.4 Counts map and model map. J0108-1431 . . . . . . . . . . . . . 85

6.5 Counts map and model map. J0953+0755 . . . . . . . . . . . . . . . 85

6.6 Counts map and model map. J0630-2834 . . . . . . . . . . . . . . 86

6.7 Counts map and model map. J1136+1551 . . . . . . . . . . . . . . 86

6.8 Counts map and model map. J0826+2637 . . . . . . . . . . . . . . 87

6.9 Log-likelihood curves for $n=2.3$. . . . . . . . . . . . . . . . 88

6.10 Log-likelihood curves for $n=4.5$. . . . . . . . . . . . . . . . 89

6.11 Log-likelihood curves for $n=6.7$. . . . . . . . . . . . . . . . . 90

7.1 Histograms of flux upper limits, for $n=2.3$, for RXJ1856-3754 . . . . 95

7.2 Histograms of flux upper limits, for $n=4.5$, for RXJ1856-3754 . . . . 96

7.3 Histograms of flux upper limits. for $n=6.7$. for RXJ1856-3754 . . . . 97

7.4 Performance comparisons of two Pass 6 IRFs considered. . . . . . . . 100

8.1 Spectra $d N_{n} / d E_{\gamma}$, at different temperatures, for $n=5 . \ldots . . . . .104$ 
A.1 Differential flux distributions for RXJ1856-3754 . . . . . . . . . . 112

A.2 Differential flux distributions for J0108-3754 . . . . . . . . . . 113

A.3 Differential flux distributions for J0953+0755 . . . . . . . . . . . 114

A.4 Differential flux distributions for source J0630-2834 . . . . . . . . 115

A.5 Differential flux distributions for J1136+1551 . . . . . . . . . 116

A.6 Differential flux distributions for J0826-2637 . . . . . . . . . . . 117

C.1 Counts map in the 1FGL catalog ROI 32 . . . . . . . . . . . . 127

D.1 Efficiencies after each successive cut is applied. . . . . . . . . . . . 131

D.2 Comparison of EvtEnergvCorr/McEnergy for two IRFs . . . . . . 133

D.3 Energv resolution for different energv variables in two different IRFs 134

D.4 Tov MC distributions of fit parameters for EvtEnergyCorr/McEnergy 135 


\section{Chapter 1}

\section{Introduction}

\subsection{Classical and Relativistic Gravity}

Physics as a science, in the modern sense, had its first well-characterized mathematical description of a natural force in gravity. During the $16^{\text {th }}$ and $17^{\text {th }}$ centuries, scholars such as Galileo, Kepler, and Newton used the language of mathematics to describe the results of empirical data on objects in space and earth. Kepler's empirical deductions from careful observations of planetary motion led to the formulation of Kepler's Laws of Motion. Newton applied the mathematical apparatus of calculus to explain Newtons' Laws, and applied them to gravity. Although gravity was described in Principia by Newton, the action at a distance idea was invoked, which he found hard to accept completely:

That gravity should be ...so that one body may act upon another at a distance through a vacuum without the mediation of anything else, by and through which their action and force may be conveyed is so to me so great an absurdity [1]...

Newton continues in his writing that was missing was a perhaps even more fundamental explanation, but which was elusive to the scientists of his day.

Centuries later, more precise astronomical observations would reveal deviations from Newtonian gravity. One such unexplained discrepancy was that the perihelion 
of Mercury precessed in excess of that predicted by Newton's theory 1 . Einstein proposed the theory of general relativity in the early $20^{\text {th }}$ century. His equations would account for the deviations in Mercury and other celestial objects. In 1919, the deflection of light was measured during a solar eclipse in the southern part of Africa by Sir Arthur Eddington, and confirmed Einstein's prediction spectacularly. The theory would also enjoy success where Newtonian physics would be inadequate, such as describing compact and dense objects such as neutron stars and black holes. General relativity (GR) was truly a revolution in the conception of gravity and physics in general. Unlike in Newton's physics, which assumed space and time could be measured in an absolute, or inertial frame of reference, space and time were relative. GR is a metric theory, in which space and time can be curved, due to matter and energy.

\subsection{Beyond Classical and Relativistic Gravity}

Despite the successes of GR, there are motivations for a theory which goes further. One such motivation lies in a unified explanation of the fundamental forces. Quantum theory has unified the electromagnetic and weak interactions at the atomic and subatomic scales, as probed by accelerator/collider experiments; on the other hand, general relativity describes gravity on astrophysical scales. One great challenge for particle physics is an explanation to reconcile the two, a grand unified theory (GUT) or a quantum theory of gravity. Theories of extra dimensions are found in the context of modern string theory to account for the unification of the fundamental forces. Theories of extra dimensions are collectively known as Kaluza-Klein (KK) theories, after Theodor Kaluza who generalized Einstein's theory in 5 dimensions to simultaneously explain electromagnetic and gravitational interactions (1919), and Oskar Klein, who described compactification of extra dimensions (1926).

It is evident from the relative strength of the forces that they are not unified according to the previous theoretical framework. The dimensionless coupling constant

\footnotetext{
${ }^{1}$ The observed perihelion is 574 arcmin/century, whereas the Newtonian calculated perihelion is $531 \mathrm{arcmin} /$ century [2].
} 
for the gravitational force is much weaker than the electromagnetic force for charged elementary particles. The gravitational coupling constant (defined in terms of electron mass) is given by $\alpha_{G}=\frac{G_{N} m_{e}^{2}}{\hbar c} \approx 1.75 \times 10^{-45}$, where $G_{N}$ is Newton's constant, $m_{e}$ is the electron mass, $\hbar$ is the reduced Planck's constant, $c$ is the speed of light; the electromagnetic fine structure constant is given by $\alpha=\frac{e^{2}}{\hbar c} \approx \frac{1}{137.03} \approx 7.297 \times 10^{-3}$. The quotient of the coupling constants shows that the repulsive electromagnetic force between two electrons is 43 orders of magnitude larger than the attractive force of gravity2. Particle physicists view the coupling constants in terms of parameters with dimensions of energy. For the case of gravity, this is the Planck mass, which is given by $M_{P}=\sqrt{\frac{\hbar c}{G_{N}}} \approx 1.22 \times 10^{19} \mathrm{GeV}$. For the electroweak interactions, the scale is $M_{\mathrm{EW}} \sim 1 \mathrm{TeV}$, which is related to the vacuum expectation value (VEV) of the Higgs boson (derived from accelerator experiments' bounds on Higgs boson mass). The large difference between these mass scales is known as the hierarchy problem.

Another problem which manifests is the apparently small value of the vacuum energy density of the universe, which is known as the cosmological constant problem [3]. According to the idea of spontaneous symmetry breaking in particle physics, we expect the cutoff scale to depend as $\Lambda \sim M_{P}^{4}$, to be of the order of $10^{76} \mathrm{GeV}[4]$. On the other hand, the observational bound on the cosmological constant is on the order of $10^{-48} \mathrm{GeV}$. There is an apparent paradox of $10^{124}$ orders of magnitude, which is even worse than the hierarchy problem.

\subsection{Shortcomings of the Standard Model}

The Standard Model (SM) of Physics has been successful at describing electromagnetic force, the weak force, and the strong nuclear forces in a unified $\mathrm{SU}(3) \times \mathrm{SU}(2) \times$ $\mathrm{U}(1)$ framework. The forces appear to be different, but due to the running of the coupling constants according to the renormalization group, the forces unify at some scale, as shown in Figure 1.3. However, the SM is not a theory of everything (TOE),

\footnotetext{
${ }^{2}$ The fact that planetary motion in the solar system can be explained by gravity without consideration of electrodynamics indicates that these bodies are electrostatically neutral, overall; on the other hand propagation of cosmic rays are hardly impacted by gravity.
} 


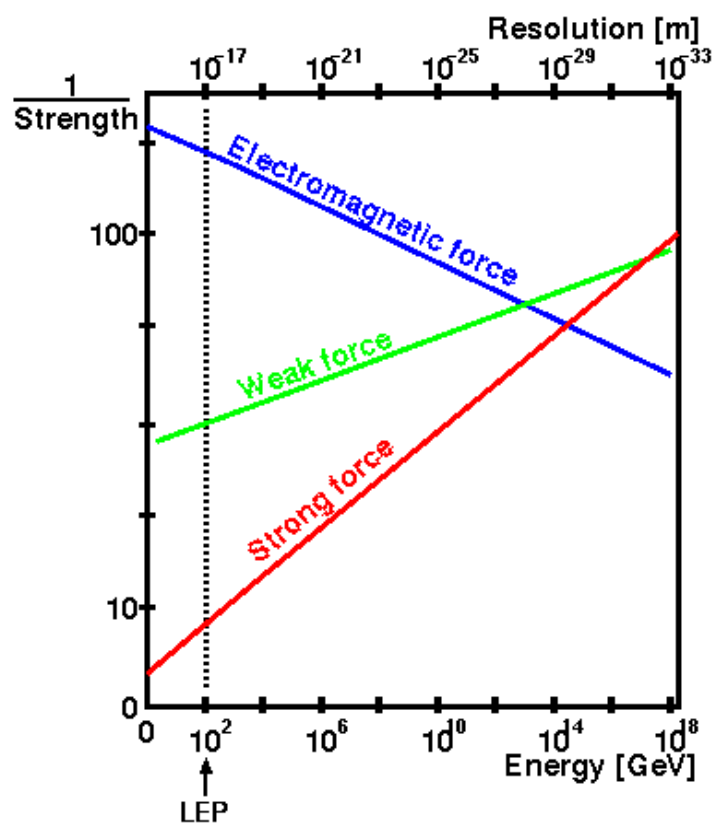

Figure 1.1: Running of the coupling constants as a function of energy []. $]$.

because it doesn't describe gravity in a fundamental way. Gravity is not unified with other forces, as the hierarchy problem makes obvious. One of the motivations for beyond the SM theories,such as supersymmetry and string theory, is to resolve this problem. In addition, the SM doesn't address the question of dark matter. Dark matter particles arise naturally in some scenarios of extra dimensions.

\subsection{Model of Large Extra Dimensions}

In 1998, Nima Arkani-Hamed and Savas Dimopoulos at Stanford University, as well as Gia Dvali (ADD), postulated a theory of large extra dimensions (LED) as an explanation for the hierarchy problem [6]. In this scenario, only the gravitational force would propagate in the $n$ extra dimensions; other forces would be restricted to the ordinary $(3+1)$-dimensional world, or 3-brane in string theory terminology 6]. For simplicity, they assumed that all the extra dimensions were compactified with the same radius $R$. If test masses are placed apart at distances less than $R$, the gravitational potential would obey a $1 / r^{n+1}$ potential. On the other hand, if 
test masses are placed apart at distances larger than $R$, the gravitational potential would return to the familiar $1 / r$ dependence. Thus, due to the presence of additional dimensions, the effective Planck scale in $(n+4)$ dimensions, $M_{D}$, would be brought closer to the $\mathrm{TeV}$ scale according to the following relation:

$$
\bar{M}_{P}^{2}=R^{n} M_{D}^{2}
$$

where the reduced Planck mass, $\bar{M}_{P}=M_{P} / \sqrt{8 \pi}=2.43 \times 10^{15} \mathrm{TeV}$. This behavior can be shown by a simple exercise of a higher dimensional version of Gauss' law, where the action is given by:

$$
S_{E}=\frac{\bar{M}_{D}^{2+n}}{2} \int d^{4} x d^{n} y \sqrt{-\operatorname{det} g} \mathcal{R}(g),
$$

where $x$ denotes the $4 \mathrm{D}$ coordinates, $y$ denotes the extra-dimensional coordinates, $g$ denotes the metric tensor, and $\mathcal{R}$ describes the curvature. Integrating over the extra dimensions, the integral over $d^{n} y$ becomes a volume in the extra dimensional space, which is proportional to $R^{n}$.

One of the most notable predictions of this theory was large dimensions at submillimeter scales. By setting $M_{P, n+4} \sim M_{\mathrm{EW}}$, they obtained:

$$
R \sim 10^{30 / n-17} \mathrm{~cm} \times\left(\frac{1 \mathrm{TeV}}{M_{\mathrm{EW}}}\right)^{1+2 / n}
$$

For the $n=1$ case, one obtains $R \sim 10^{13} \mathrm{~cm}$, so $n=1$ was quickly excluded empirically in this theory. For $n=2$, the size of the extra dimension is on the order of $R \sim 100 \mu \mathrm{m}-1 \mathrm{~mm}$, so this attracted interest at the time of publication. It is small enough to have not been detected at the time the paper was written, but large enough to be within the accessible range of experiments probing deviations from the inverse square law, as described in Section 1.6.1.

A prediction of the ADD model is that the gravitational force is mediated by a gauge boson called a Kaluza-Klein (KK) graviton. Unlike the graviton of GR, this graviton has a mass. The KK excitations of the graviton, denoted as $G_{K K}$, are expected to form a tower of states, with a mass observable in the 3-brane which arises 
from the momentum of the zero-mass KK graviton in the hidden extra dimensions.

In $4 \mathrm{D}$ space, the metric tensor is given by the familiar Minkowski metric:

$$
\eta_{\mu \nu}=\left(\begin{array}{cccc}
1 & 0 & 0 & 0 \\
0 & -1 & 0 & 0 \\
0 & 0 & -1 & 0 \\
0 & 0 & 0 & -1
\end{array}\right)
$$

which corresponds to the equation:

$$
d s^{2}=c^{2} d t^{2}-d x^{2}-d y^{2}-d z^{2} .
$$

In the presence of extra dimensions, the relevant tensor becomes

$$
G_{M N}=\eta_{M N}+\frac{h_{M N}}{M_{4+n}^{(n+2) / 2}}
$$

where $M, N$ are indexed into the $4 \mathrm{D}$ space and the extra dimensional space. The tensor for $h^{\mu \nu}$ can be expressed as:

$$
h^{\mu \nu}=\sum_{j} \frac{1}{\sqrt{R^{n}}} h^{\mu \nu, \vec{j}} \exp \left(\frac{i \vec{j} \cdot \vec{y}}{R}\right)
$$

where $\vec{j}$ is the $n$-dimensional momentum vector, $\vec{y}$ is the $n$-dimensional coordinate vector, and $R$ is the radii of the extra dimensions [7] [8]. It follows that the mass arising in the 3-brane can be written as:

$$
m_{j}^{2}=\frac{|\vec{j}|^{2}}{R^{2}}+m_{0}^{2}
$$

where $m_{0}$ is the mass of the zero mode. 


\section{Derivation of the Mass Tower}

It is instructive to show a pseudo-derivation of the KK mass tower. following the method of Rizzo $[9]^{3}$. Suppose there is only one additional space-like dimension, $y$.

$$
p^{2}=g_{A B} p^{A} p^{B}=p_{0}^{2}-\vec{p}^{2}-p_{5}^{2}
$$

where the metric tensor $g_{A B}=\operatorname{diag}(1,-1,-1,-1,-1)$. According to Lorentz invariance,

$$
p_{0}^{2}-\vec{p}^{2}=p_{\mu} p^{\mu}=m^{2}
$$

The 5D Klein-Gordon equation is given by,

$$
\partial_{A} \partial^{A} \Phi=\left(\partial_{\mu} \partial^{\mu}-\partial_{y}^{2}\right) \Phi(x, y)=0
$$

Separating variables, and summing over the modes, we obtain the following form for $\Phi$ :

$$
\Phi=\sum_{l} \chi_{l}(y) \Phi_{l}(x)
$$

Plugging this from for $\Phi$ back into the Klein-Gordon equation, we obtain the following form:

$$
\sum_{n}\left(\chi_{l} \partial_{\mu} \partial^{\mu} \phi_{l}-\phi_{l} \partial_{y}^{2} \chi_{l}\right)
$$

With the relation that

$$
\partial_{y}^{2} \chi_{l}=-m_{l}^{2} \chi_{l}
$$

the following can be obtained

$$
\sum_{l} \chi_{l}\left(\partial_{\mu} \partial^{\mu}+m_{l}^{2}\right) \phi_{l}=0
$$

The preceding equation expresses that the $4 \mathrm{D}$ scalar fields, $\phi_{l}$, have discrete

\footnotetext{
${ }^{3}$ I call this pseudo because the rigorous way is to use the action, the integral of the Lagrangian.
} 
masses. The fields $\chi_{l}$ are the wave functions of the various KK states in the $5^{\text {th }}$ dimension and may be expressed as simple harmonic functions:

$$
\chi_{l}=A_{l} e^{i m_{l} y}+B_{l} e^{-i m_{l} y}
$$

Since the extra dimension is compact, and not infinite, boundary conditions apply, such that the following constraint holds:

$$
0<y<\pi L
$$

The solutions for $\chi$ are given by

$$
\chi_{l} \propto \sin (l y / L)
$$

so the masses are quantized as:

$$
m_{l}^{2}=l^{2} / L^{2} .
$$

This argument can easily be extended to the case of $n$ extra dimensions, where

$$
0<y_{i}<\pi L,
$$

where $i=1 . . n$.

In this case, if

$$
\vec{j}=\sum_{i} l_{i} \hat{e}_{i}
$$

then

$$
m_{j}^{2}=|\vec{j}|^{2} / L^{2}
$$

which is similar to the result in equation (1.8), with $L \propto R$, but not including the zero-point mass term. 


\subsection{Graviton Emission}

Arkani-Hamed, Dimopoulos, and Dvali further proposed that the process of graviton emission occurs within supernova cores. This competes with the process of neutrino emission as a cooling mechanism in radiative energy transfer, while neutrino emission occurs by diffusion due to the exotic conditions of densities of order $10^{14} \mathrm{~g} \mathrm{~cm}^{-3}$, and temperatures of approximately $30 \mathrm{MeV}$. Specifically in the case of SN 1987A, a core-collapse supernova event whose neutrino flux was monitored terrestrially by the Kamiokande detector in Japan, they argued that the observed signal was consistent with the graviton emission scenario they proposed [10]. Long duration and the lowrate indicated it was a diffusion process of neutrinos, rather than free streaming. They also argued that the dominant process of KK graviton production within the core of the supernova, or proto-neutron star, was nucleon-nucleon bremsstrahlung, $N N \rightarrow N N h$, with a cross section on order 30 millibarns. First, they calculated the graviton luminosity, $\mathcal{L}_{\text {grav }}$, from $N N$ bremsstrahlung as:

$$
\mathcal{L}_{\text {grav }} \sim M_{\mathrm{SN}} \cdot \frac{n_{N}^{2}}{\rho} \cdot 30 \mathrm{mb} \cdot\left(\frac{T}{M_{n+4}}\right)^{n+2} T
$$

where $M_{\mathrm{SN}} \sim 1.6 M_{\odot} \sim 10^{57} \mathrm{GeV}$, is the supernova mass, $n_{N} \sim 10^{-3} \mathrm{GeV}^{3}$ is the nucleon number density within the supernova core, the matter density $\rho \sim 10^{-4} \mathrm{GeV}^{4}$, and $T \sim 30 \mathrm{MeV}$ is the temperature of the supernova core. They equated the $\mathcal{L}_{\text {grav }}$ with release of gravitational binding energy of $10^{53} \mathrm{ergs} / \mathrm{s}$, and then quoted a lower bound for 2 additional dimensions, with $M_{P, 6}$ of $30 \mathrm{TeV}$.

When making predictions about LED, ADD assumed that the nucleon-nucleon $(N N)$ bremsstrahlung was mediated by pion-exchange via the strong force 6, 10$]$. Cullen and Perelstein assumed this as well when placing limits from SN1987A 11]. On the other hand, Hanhart, Reddy and Savage used the simplifying assumptions of an effective theory. Assuming gravitons were emitted with energy lower than the energy of the incoming nucleons, i.e., soft-radiation approximation, and keeping leading order

\footnotetext{
${ }^{4}$ Another process for producing gravitons is the gravi-Primakoff process, in which a graviton is emitted from a photon in the EM field of a nucleus, but which was shown by ADD to yield less restrictive limits on LED and will not be considered in calculations.
} 
terms in nucleon energy, they were able relate the quantum amplitude for the process to the on-shell $N N$ scattering amplitude. This is done in analogy to Weinberg's calculation for massless graviton emission bremsstrahlung in 4 dimensions.

\subsection{Previous Experimental Constraints}

\subsubsection{Tabletop Experiments of the Inverse Square Force Law}

On small length scales, deviations from the inverse square force law (ISL) have been studied. Deviations from the ISL, for masses of mass $m_{1}$ and $m_{2}$ at a distance $r$, have been parametrized as a Newtonian interaction plus Yukawa-type term:

$$
V(r)=-G_{N} \frac{m_{1} m_{2}}{r}[1+\alpha \exp (-r / \lambda)]
$$

The Yukawa term is an appropriate choice, since it arises from exchange of a boson of mass $m=\hbar /(c \lambda)$.

Tabletop experiments at the sub-millimeter and at the sub-micron scales have placed useful constraints and are continually undergoing improvements in resolution. When comparing the gravitational force law with the $n$ toroidally-compactified extra dimensions, the parameter $\alpha=8 n / 3$ and $\lambda$, the Compton wavelength of the first KK mode, is equal to $R$. One of the most sensitive experiments to KK gravitons, the University of Washington experiments, utilized torsion pendulums and rotating attractors to probe deviations from ISL[12, 13]. For the $n=2$ case, from Figure 1.2, $R<37 \mu \mathrm{m}$ and $M_{D}>3.6 \mathrm{TeV}$. At $95 \%$ C.L., they reported no apparent deviations from ISL.

Another sensitive experiment, from a Stanford group of David Weld, Jing Xia, Blas Cabrera, and Aharon Kapitulnik, used cryogenic micro-cantilevers to measure forces of the order of $10^{-18} \mathrm{~N}[14$. The Stanford group reported no deviations from ISL at $5 \mu \mathrm{m}$. The bounds in $\alpha-\lambda$ space, shown in Figure 1.2, were determined from computing the value of $\alpha$ that would produce that force at each $\lambda$, given the experimental geometry. 


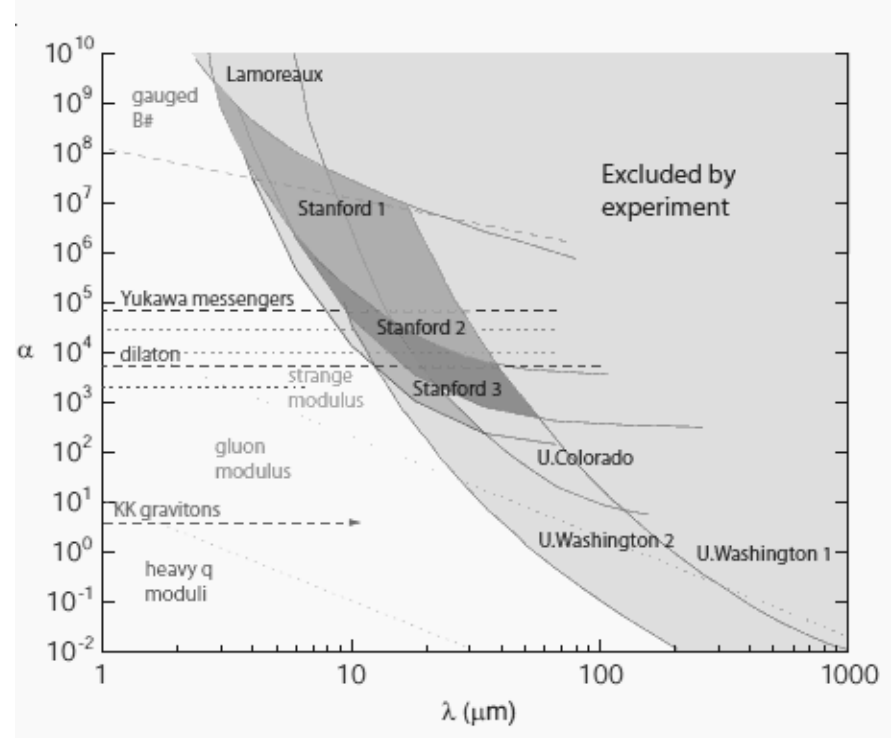

Figure 1.2: Deviations from Newtonian gravity parametrized with a Yukawa-type interaction according to equation (1.24). Boundaries from 95\% exclusion regions from various experiments (shaded areas) are shown from different regions as dark curves [14].

\subsubsection{Collider Constraints}

\section{TeVatron Constraints}

The collision of standard model particles can lead to missing energy signatures at colliders. At LEP (CERN), the following processes have been investigated:

$$
\begin{aligned}
& e^{+} e^{-} \rightarrow \gamma+\mathbb{E} \\
& e^{+} e^{-} \rightarrow Z+\mathbb{E}
\end{aligned}
$$

At TeVatron (Fermilab), diphoton and dilepton modes have been investigated with $\mathrm{CDF}$ and $\mathrm{D} \varnothing$ :

$$
\begin{gathered}
p \bar{p} \rightarrow \gamma \gamma \\
p \bar{p} \rightarrow e^{+} e^{-} .
\end{gathered}
$$

These processes are mediated by virtual graviton exchange. In addition, states with 


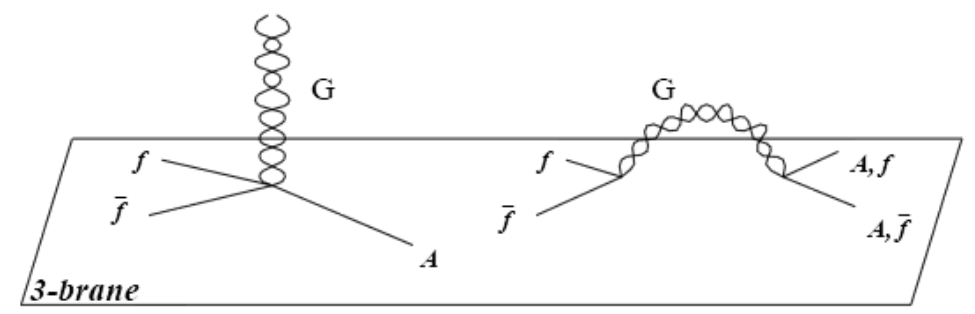

Figure 1.3: Left, schematic of KK graviton $(G)$ emission, from 15]. Right, schematic of graviton exchange. $f$ represents SM quarks and leptons, while $A$ are the gauge bosons $\gamma, W, Z$, or gluon. The vertical direction indicates the space in extra dimensions, out of $4 \mathrm{D}$ space.

missing energy were probed with $\mathrm{CDF}$ and $\mathrm{D} \varnothing$. In these modes, the final-state hadronic jet or photon recoils against the graviton, which is not detected and results in missing transverse energy, $\mathbb{F}_{T}$ :

$$
\begin{gathered}
p \bar{p} \rightarrow \text { jet }+\mathbb{F}_{T} \\
p \bar{p} \rightarrow \gamma+\mathbb{F}_{T}
\end{gathered}
$$

The processes for virtual graviton exchange and graviton emission are depicted in Figure 1.3. Graviton exchange processes introduce an ultraviolet cutoff $\Lambda$,

$$
\mathcal{L}=\frac{4 \lambda}{\Lambda^{4}} T_{\mu \nu}^{i} T_{f}^{\mu \nu}
$$

where $\lambda= \pm 1$ and $T_{\mu \nu}^{i} T_{f}^{\mu \nu}$ is a dimension- 8 operator introduced by tree-level graviton exchange, with $T^{\mu \nu}$ being the SM energy-momentum tensor 16 .

\section{Constraints from LHC}

Experiments at LHC, a proton-proton collider, including ATLAS and CMS, have been searching for extra dimensions. Investigators have been studying the processes:

$$
\begin{gathered}
p p \rightarrow \gamma \gamma \\
p p \rightarrow \text { jet }+ \text { jet }
\end{gathered}
$$


Figure 1.4 shows regions of parameter space for the mass scale $M_{D}$, where $\Lambda$ is an ultraviolet cutoff parameter, of the order unity, arising from graviton exchange processes. The ratio $\Lambda / M_{D}$ parametrizes how strongly or weakly coupled quantum gravity is, and therefore controls the unknown relative importance of tree-level versus loop graviton effects.

\subsubsection{Astrophysical Constraints}

In 2003, Hannestad and Raffelt (HR) subsequently extended the analysis of KK graviton emission from supernovae, and argued that the KK gravitons would be gravitationally trapped from the time of production during supernova core collapse, so that neutron stars would be surrounded in a "cloud" of gravitons. They postulated that the decay of gravitons surrounding neutron stars would lead to a flux of photons measurable by gamma-ray observatories, such as EGRET and Fermi (being developed at the time). Based on EGRET data, their analysis placed more restrictive limits than available from the SN1987A signal; that is, they increased the lower bounds for the mass scale, $M_{D}$, and decreased the upper bounds on the compactification radius $R$ 18]. Using similar assumptions in this analysis, it will be shown that more restrictive limits can be obtained with the Fermi Large Area Telescope (LAT). A schematic depicting a neutron star, KK graviton cloud, gamma rays, and the Fermi-LAT is shown in Figure 1.5.

Other ideas for constraining extra dimension with KK gravitons have been investigated. In 2004, Cassé, Paul, Sigl, and Bertone set limits from averaging over a population of neutron stars in the galactic center 19]. They inferred the number of neutron stars in the galactic center using the Salpeter mass function. Using the distance of the galactic center of $8 \mathrm{kpc}$, and the EGRET flux from the galactic center, they were able to obtain a more restrictive limit than Hannestad and Raffelt. Limits can also be placed from measurements of the diffuse gamma-ray background, based on the assumption that KK gravitons were formed in the early universe, as will be discussed in the Conclusions. 

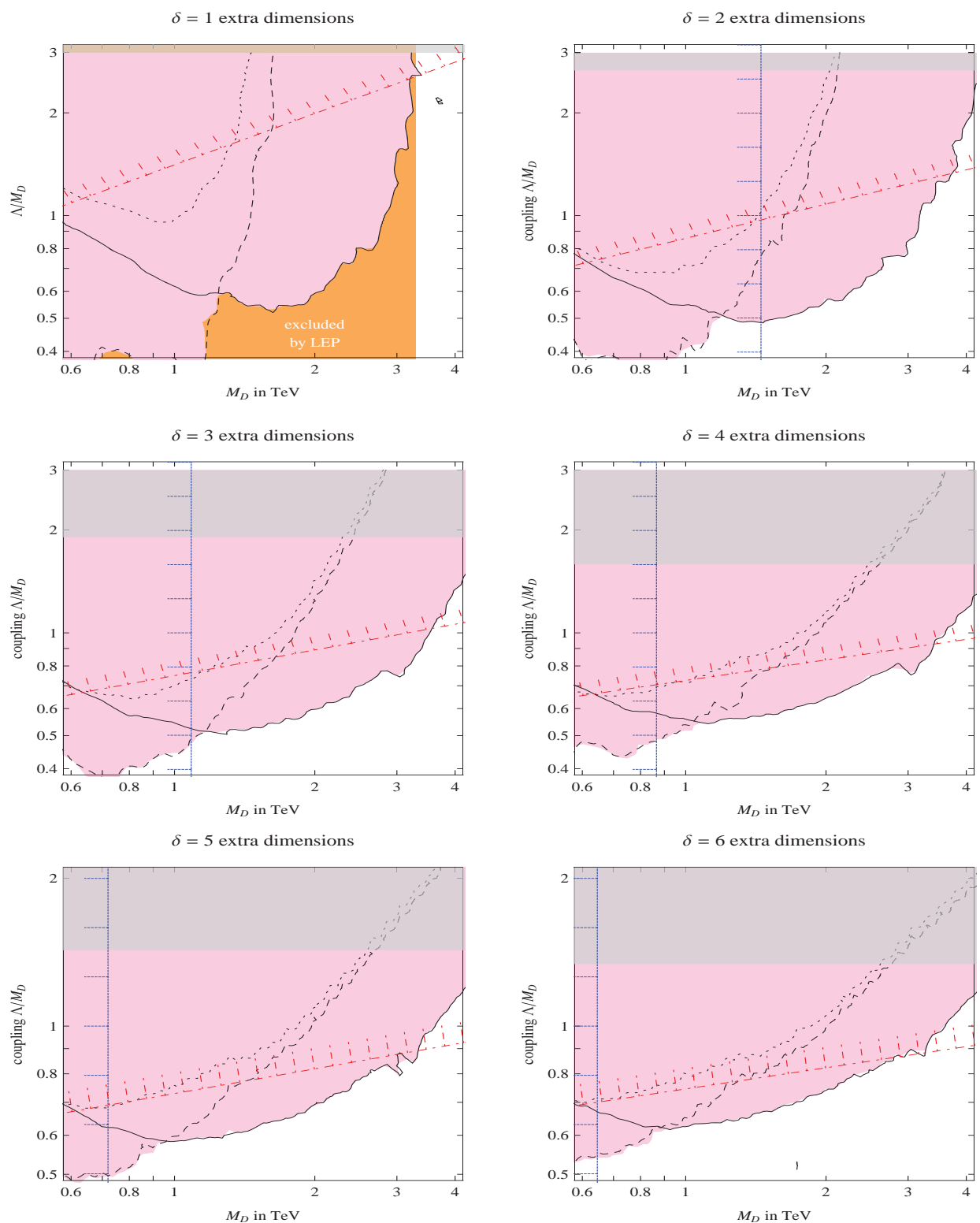

Figure 1.4: $95 \%$ confidence level limits on the variable $M_{D}$, presented as a regions of $\Lambda / M_{D}$ vs $M_{D}$ [17]. The shaded region shows the excluded region. The vertical blue line shows the combined TeVatron-LEP bound from graviton emission, ignoring the dependence on $\Lambda$. The red line is the LEP bound on loop graviton exchange, estimated according to naïve dimensional analysis. The black solid curve shown the bounds from CMS, while the black dashed curve shows the bounds from ATLAS. 


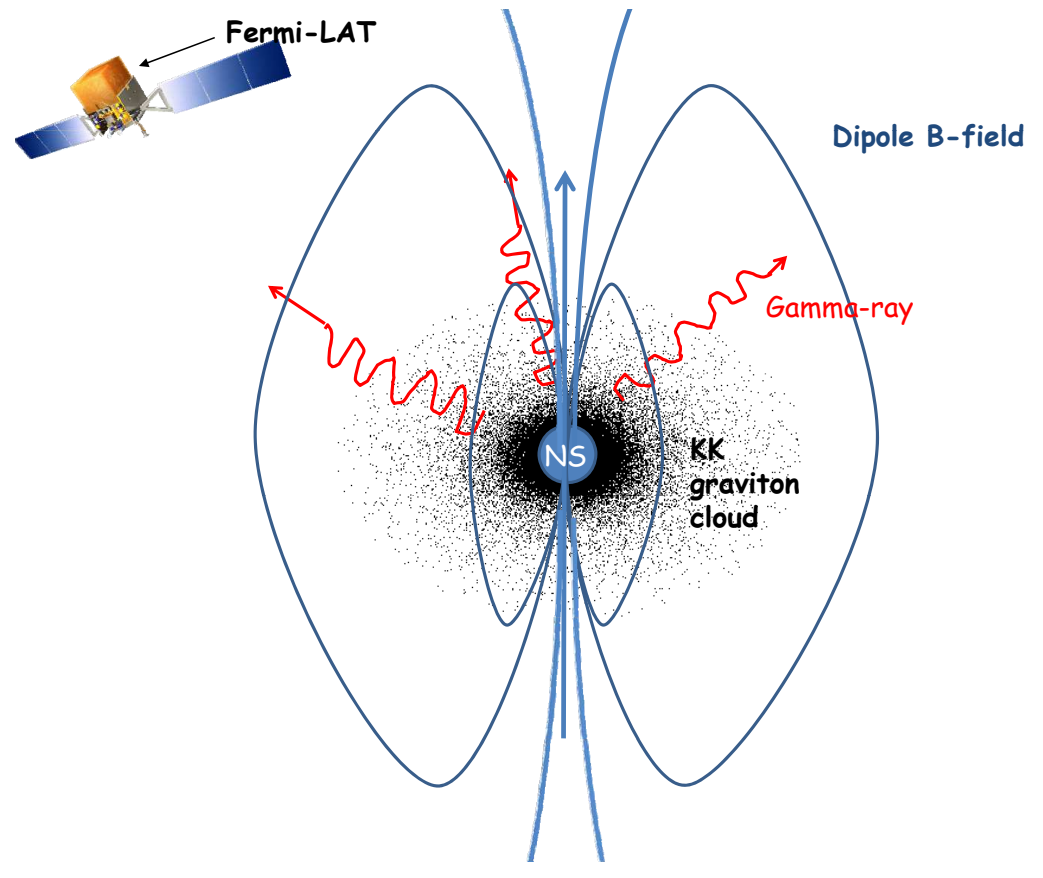

Figure 1.5: A schematic depicting a neutron star (NS), dipole magnetic field lines, a surrounding KK graviton cloud, gamma rays arising from KK graviton decays, and the Fermi-LAT role in observing gamma-rays. 


\subsection{Other Extra Dimension Scenarios}

There exist several proposed theories of extra dimensions under active consideration. The ADD model is only one such model, and one of the earliest proposed. It is worth mentioning that there are other models proposed in the late 1990s, including the Randall-Sundrum (RS) model

\subsubsection{Randall-Sundrum Model}

The RS model generates the mass hierarchy in a different mechanism. According to this scenario, in which there is only one additional "small" dimension along with the ordinary $3+1$ space-time dimensions, the metric of the usual 4 dimensional space-time is multiplied with an exponential "warp" factor [20]. The line element, with the 4D coordinates $x^{\mu}$ and 5D-coordinate $y$, is given by:

$$
d s^{2}=\exp (-2 k|y|) \eta_{\mu \nu} d x^{\mu} d x^{\nu}-d y^{2}
$$

where $1 / k$ is of the order of the $5 \mathrm{D}$ Planck mass.

The Planck mass in this model is given by:

$$
M_{D}^{2}=(1-\exp (-2 \pi k R)) M_{P}^{3} / k
$$

and the masses of the modes are given by:

$$
m_{N}=k e^{-k R} j_{n}
$$

where $j_{n}$ are the roots of the Bessel function, $J_{1}\left(j_{n}\right)=0$.

The previous equation implies that the mass splittings between graviton modes are of $\mathrm{TeV}$ scale.

\subsubsection{LED Extra Dimensions with Warping}

Warped extra dimensions can be viewed as a limiting case of ADD extra dimensions in the case of $n=1,2$, under certain assumptions, as proposed by Patrick Fox [21]. 
According to this idea, the correction to the gravitational coupling $\kappa=\sqrt{16 \pi G_{N}}$, is

$$
\kappa \rightarrow \kappa \sqrt{\left(1+\frac{5}{2} k_{W} R^{n}\right)} .
$$

where $k_{W}$ is a constant related to the degree of warping; $k_{W}=0$ recovers the nonwarped LED scenario.

\subsection{Universal Extra Dimensions}

Another model is the Universal Extra Dimensions (UED) theory, where the SM fields propagate in all dimensions. This model enjoys current interest due to predictions of a signal within the reach of TeVatron and LHC, and of a Lightest Kaluza-Klein Particle (LKP) which has properties suitable for a dark matter candidate [22] . 


\section{Chapter 2}

\section{Experimental Considerations}

\subsection{The Fermi-LAT Instrument}

The Large Area Telescope (LAT) on the Fermi mission consists of 4 main active units: the Anti-Coincidence Detector (ACD), the tracker (Tkr), calorimeter (Cal), and data acquisition system (DAC). The ACD is composed of plastic scintillator which is segmented into tiles on top and ribbons on the sides surrounding the $4 \times 4$ array of 16 towers. Each tower consists of Tkr module and a Cal module. The DAC has elements in each tower and more general data processing units th

The LAT is essentially a $\gamma$-ray pair-conversion telescope with a precision convertertracker to measure photon direction, and with a calorimeter to measure the energy.

Gamma rays may pair-produce an electron-positron pair at point known as the vertex. Electromagnetic showers may then be produced downwards from the vertex and energy and position of the shower profile may be measured in the layers of the tracker and calorimeter below as depicted in Fig. 2.1. The LAT has a total mass of $2789 \mathrm{~kg}$ and dimensions of $1.8 \mathrm{~m} \times 1.8 \mathrm{~m} \times 0.72 \mathrm{~m}$.

\subsubsection{Tracker}

The tracker consists of 18 layers per tower The 16 top layers contain embedded tungsten foil converter planes, sandwiched between $2 \mathrm{X}$-oriented planes or 2 Y-oriented 


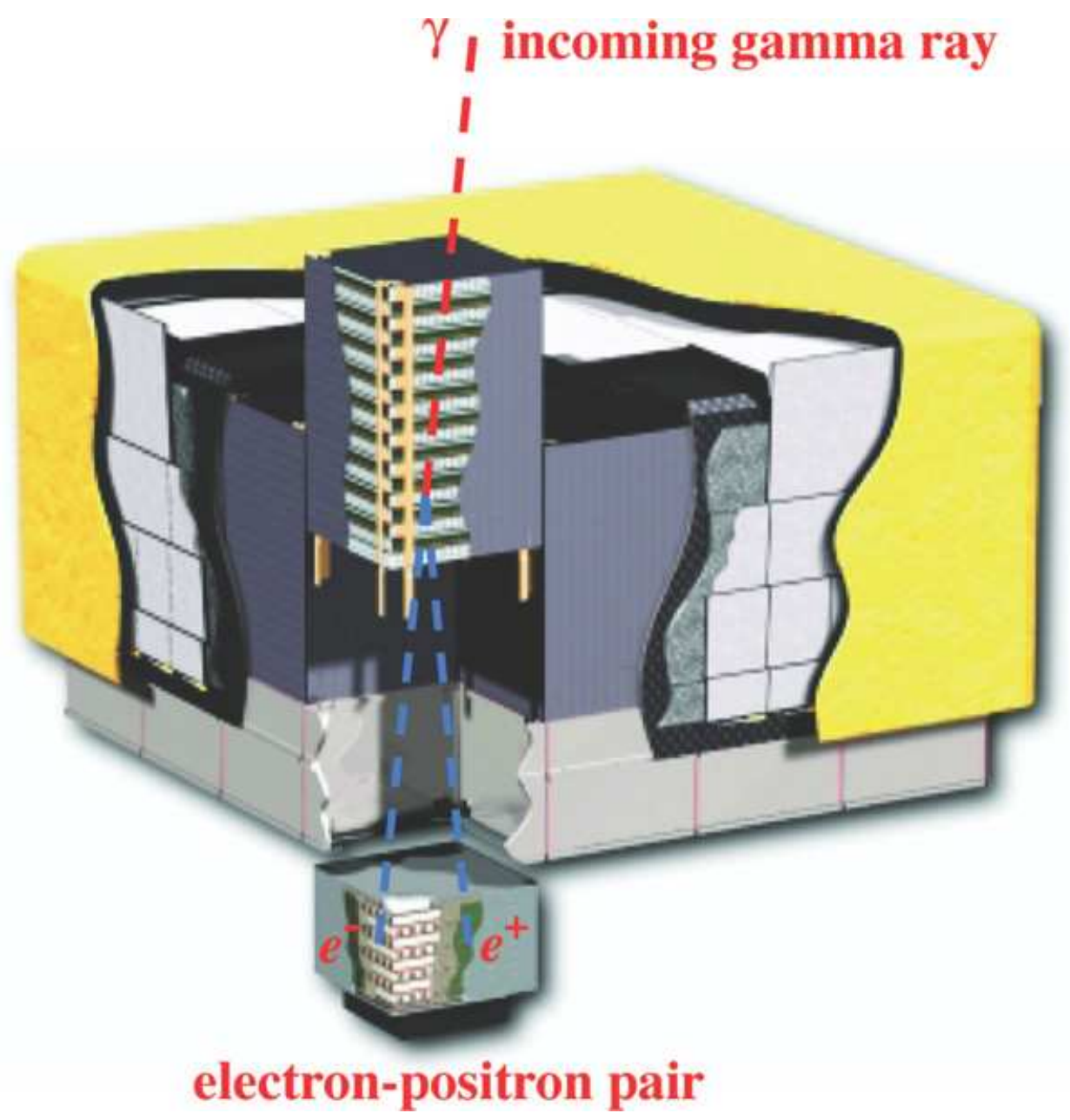

Figure 2.1: A schematic of the LAT and pair production. The yellow layer is the micrometeorite shield, the white layers are the ACD tiles. The raised module is a tower of a tracker module, and the lowered module is a calorimeter module. 


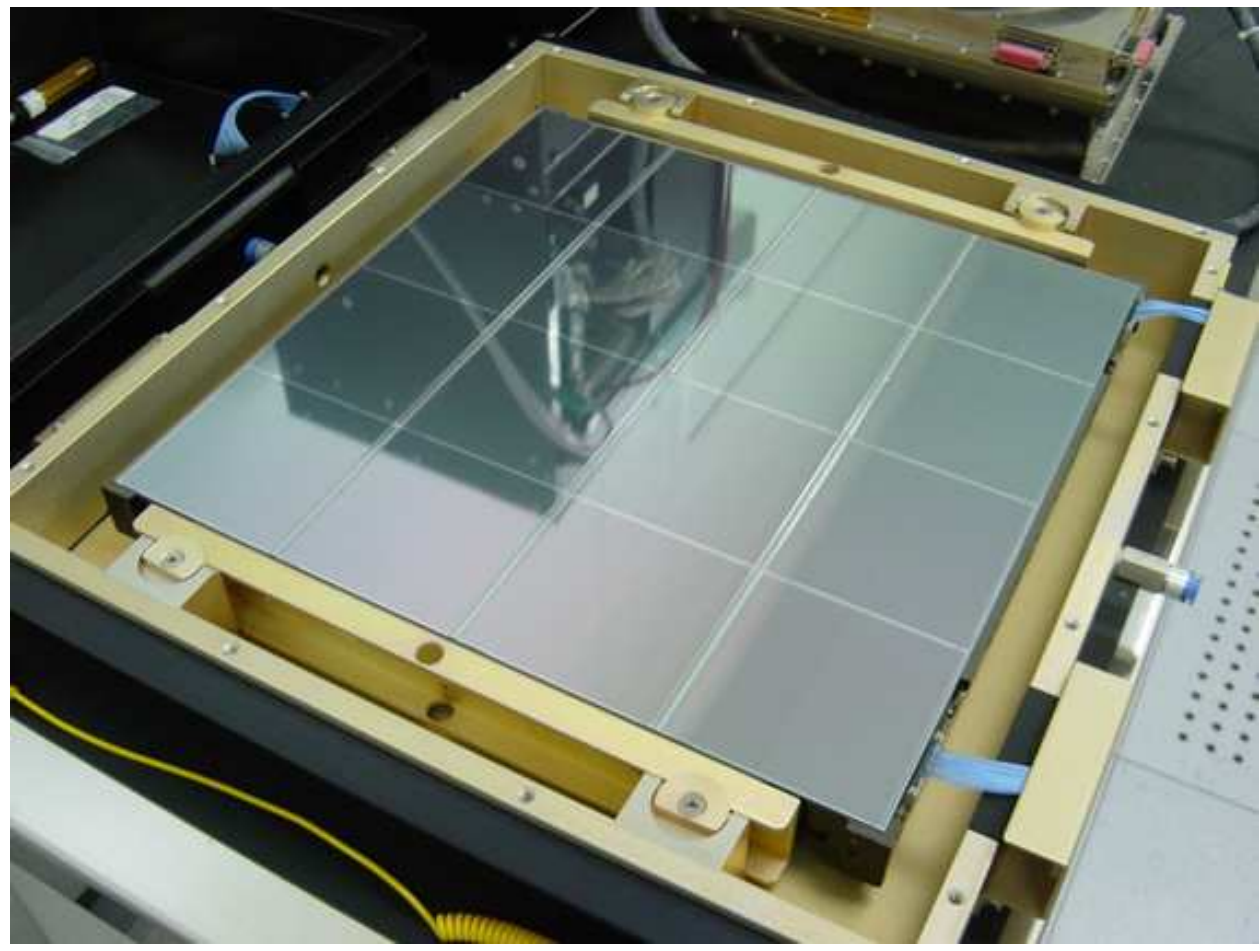

Figure 2.2: A single tracker tray

planes of silicon strip detectors (SSDs) . Tungsten was chosen as a high- $Z$ (atomic number) material to increase pair production efficiency. The top 12 layers, known as the front section, each contain a thin tungsten foil of 0.03 radiation lengths, i.e., $0.03 X_{0}$. The next 4 layers, known as the back section, each contain a thick tungsten foil of $0.18 X_{0}$.

The active region of each tracker plane consists of 16 SSDs. Each SSD has 384 individual strips. Four SSDs were bonded to make a "ladder," with the four SSDs in a given ladder joined mechanically and electrically into 384 long strips. Strips are spaced at $228 \mu \mathrm{m}$ pitch. Four ladders per plane laid side-by-side comprise a total of 1536 strips per plane. Each plane is about $360 \mathrm{~mm}$ by $360 \mathrm{~mm}$ in area. The tracker planes are supported by carbon-composite assemblies known as trays (Figure 2.2). A tray with detector strips physically parallel to the $Y$ axis is called an $X$ tray: it measures the $X$ coordinate. Readout electronics are located on the sides of the trays 


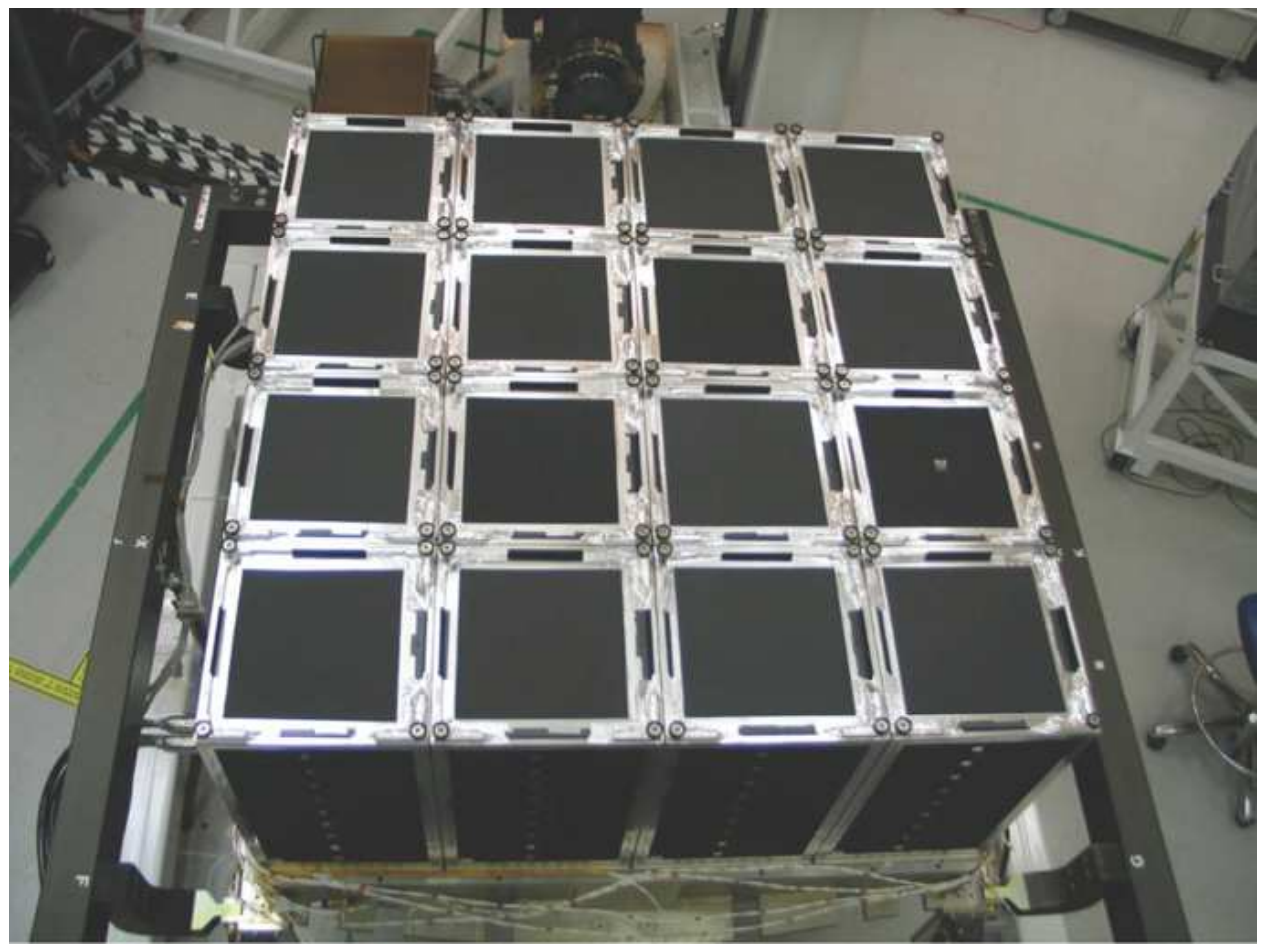

Figure 2.3: Above view of the $4 \times 4$ tracker array before integration with the ACD.

Position and energy measurements in the tracker are made with SSDs. In addition, the tracker is responsible for one of the major triggers of the LAT. A primary trigger is derived from a coincidence of successive layers of $3(X, Y)$ planes. The tracker has an aspect ratio, i.e., height/width, of 0.4: this provides a large field of view (FoV) of 2.4 steradians [23] and effective area.

\subsubsection{Calorimeter}

Each calorimeter module is segmented into 96 CsI crystals in a hodoscopic (alternating $x-y)$ configuration of 8 layers of 12 crystals, as shown in Figure 2.5. A calorimeter crystal makes coordinate measurements along its principal axis, unlike the tracker; each crystal has dimensions of $27 \mathrm{~mm} \times 20 \mathrm{~mm} \times 326 \mathrm{~mm}$;

The calorimeter measures the energy deposition due to the electromagnetic particle shower and spatially images the shower development. The transverse dimensions 


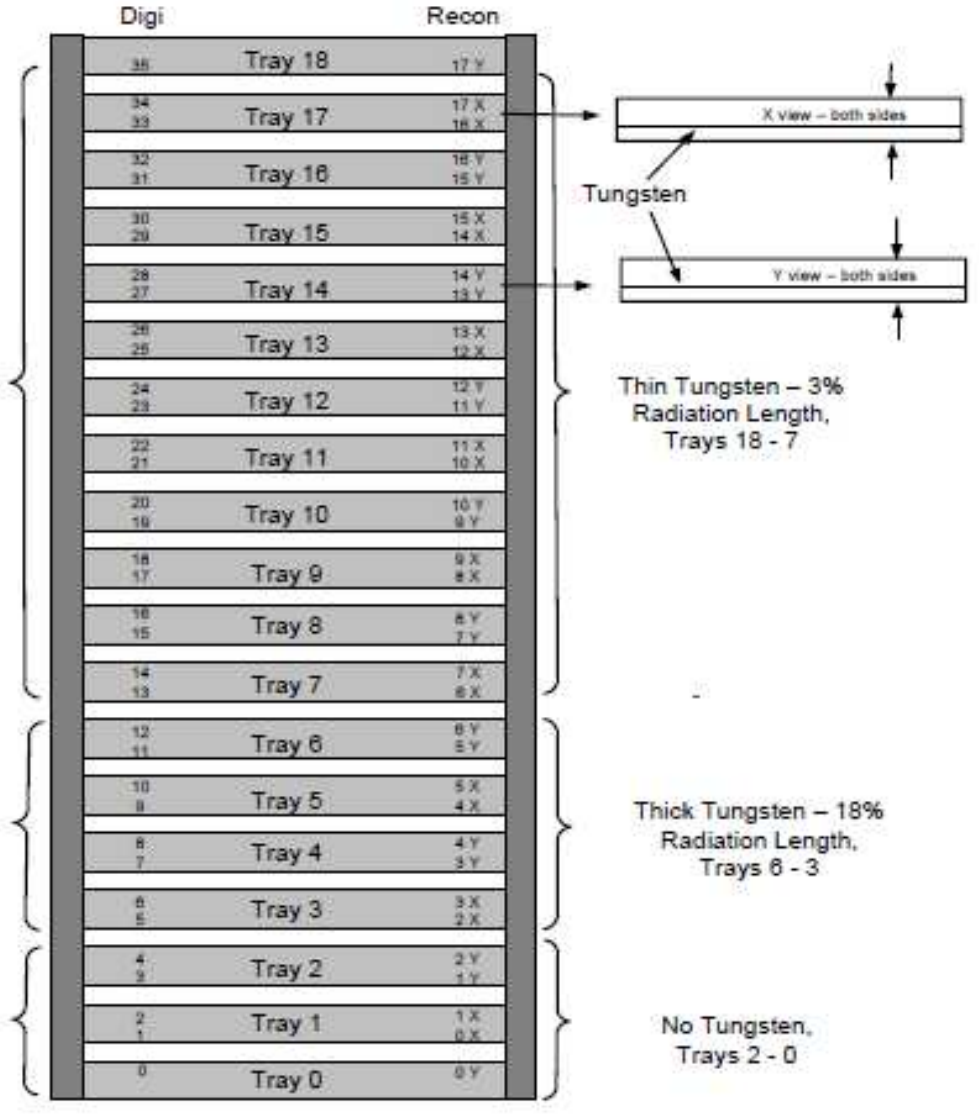

Figure 2.4: Schematic of tracker layers . The top layers of SSDs (front section) are interleaved with thin layers of tungsten, while the back layers of SSDs (back section) are interleaved with thick layers of tungsten. 


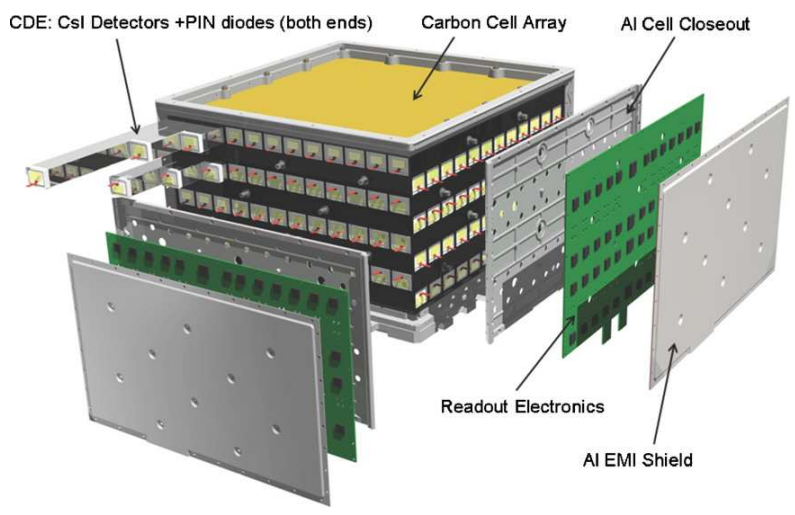

Figure 2.5: Schematic of calorimeter, cutaway view, with components labeled.

of each crystal are comparable to radiation length of CsI $\left(X_{0}=18.6 \mathrm{~mm}\right)$ and Molière radius for electromagnetic showers. The vertical depth of the calorimeter is $8.6 X_{0}$ of CsI. The level of segmentation is adequate for spatial imaging of the shower profile and reconstruction of its direction. Each crystal is optically isolated from the others. At the ends of each crystal are PIN photodiodes, of area $147 \mathrm{~mm}^{2}$ and $25 \mathrm{~mm}^{2}$. They measure transmitted scintillation light: the large photodiodes cover the energy range $2 \mathrm{MeV}$ - $1.6 \mathrm{GeV}$, while the small photodiodes cover the range $100 \mathrm{MeV}-70 \mathrm{GeV}$.

\subsubsection{Anti-Coincidence Detector}

The Anti-Coincidence detector provides charged-particle background rejection; its charged particle detection efficiency is $99.97 \%$ averaged over the area of the ACD for particles entering the LAT. The ACD, composed of plastic scintillator, is segmented into tiles on top and ribbons on the sides, as shown in Figure 2.6. Plastic scintillator was chosen for reliability, efficiency, and history of use in space missions. The ACD was designed as segmented, unlike EGRET, to prevent false veto. When a primary incident gamma-ray produces an electromagnetic shower, secondary X-ray photons from the shower may Compton scatter in the ACD and create false veto signals in the ACD, which is called backsplash. Segmentation suppresses backsplash by allowing only the ACD segment nearby the incident photon to be considered. Scintillation light from each tile is collected from wavelength shifting fibers coupled to 2 photomultiplier 


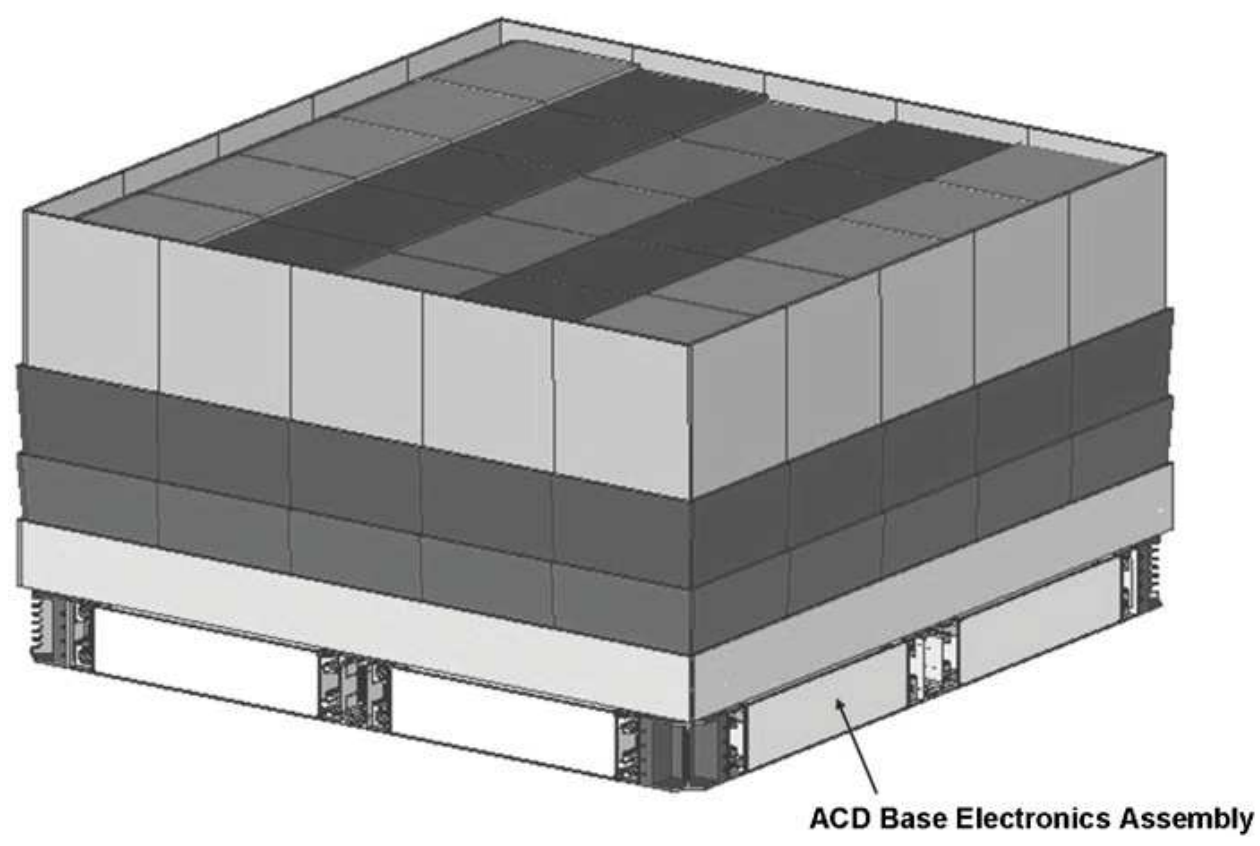

Figure 2.6: Schematic view of ACD wrapped around the tracker tower assembly and the cal assembly.

tubes (PMTs). A light micrometeoroid shield surrounds the entire ACD.

\subsubsection{LAT Data Acquisition and Electronics Modules}

The LAT Data Acquisition System (DAC) is comprised of various electronics modules as shown in Figure 2.7. There is a Tower Electronic Module (EM) for each tower calorimeter pair. There is a GASU, which interfaces to each TEM of the ACD Electronics Module.

\subsubsection{Event Reconstruction in the LAT}

Raw data from the ACD, tracker, and LAT are processed with event reconstruction algorithms. Event reconstruction of tracks is heavily dependent on MC simulations. The three main components involve the following major steps: Track-finding in the tracker based the coordinates of hit tracker strips; Extrapolating a track to the 


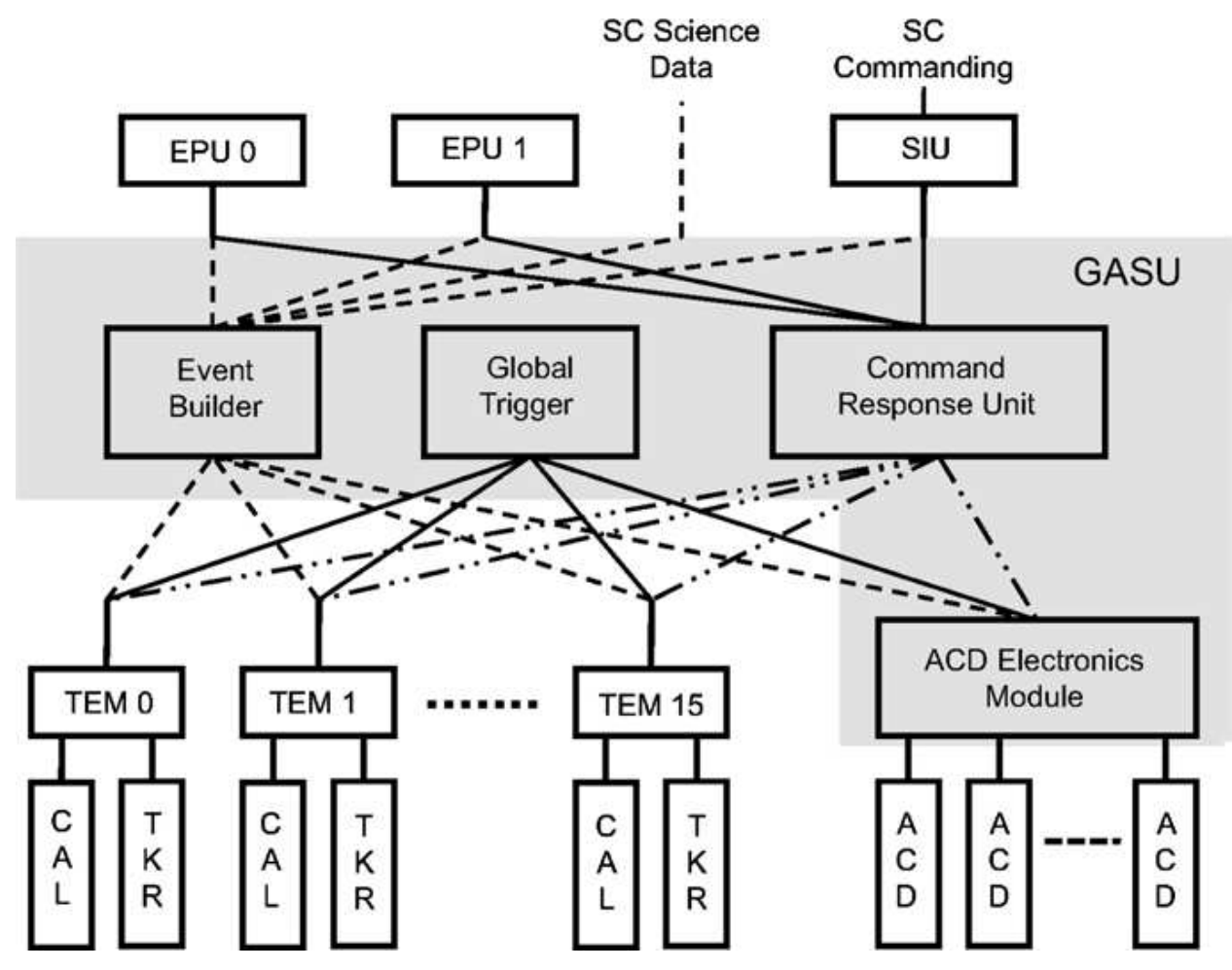

Figure 2.7: Schematic view of electronics modules for the LAT. 

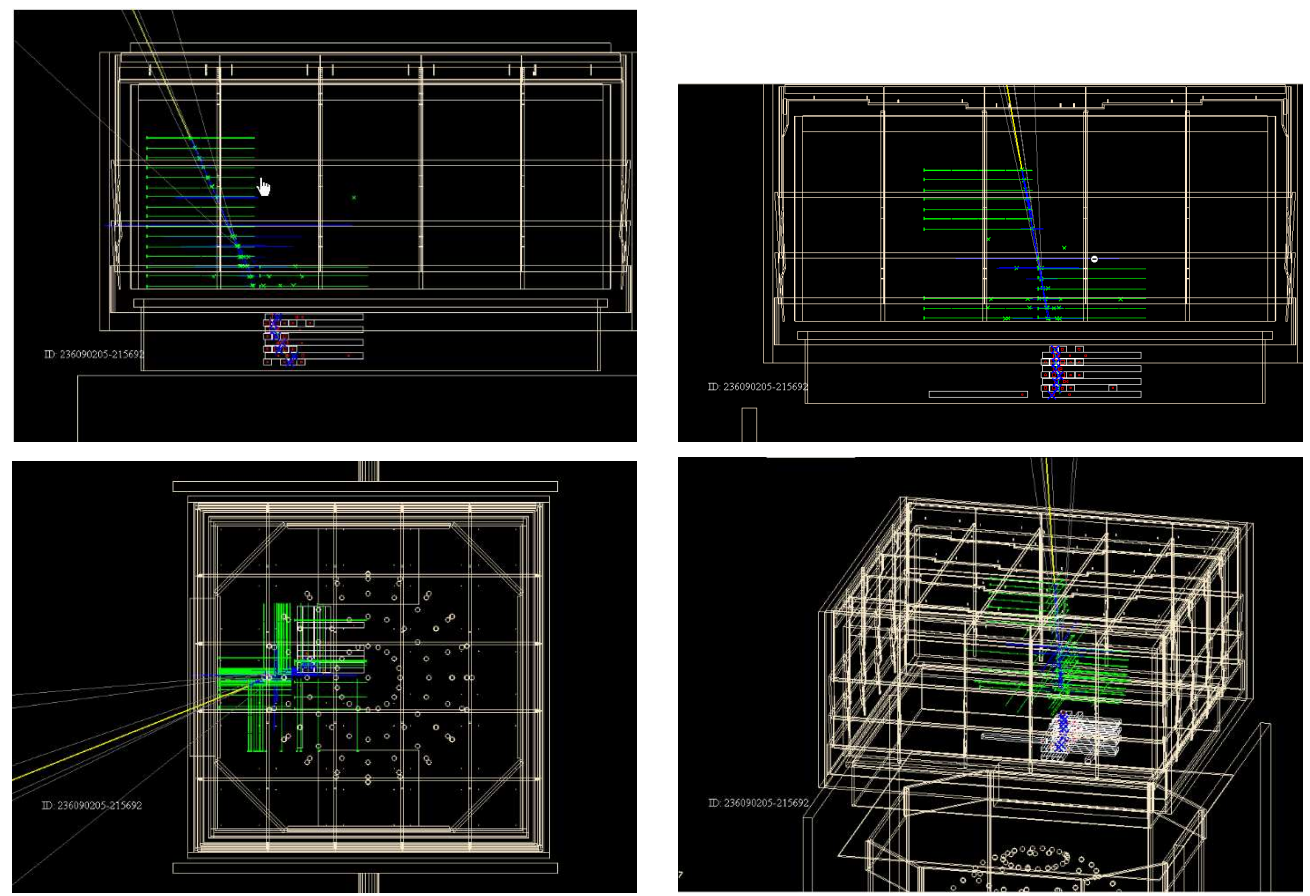

Figure 2.8: Schematic diagrams of a diffuse class event interacting in the LAT, as visualized by the WIRED event display, in different projections. Top left: $X Z$ projection. Top right: $Y Z$-projection. Bottom left: $X Y$-projection. Bottom right: rotated view. Highlighted regions of the tracker and calorimeter (in green and black, respectively) indicate energy deposition or localization in an active detector region.

calorimeter based on energy deposition pattern; Assigning a vertex location based on the 2 best tracks.

Reconstruction of the energy of each events begins with converting raw digitized voltage signals into energy measurements with the appropriate pedestals and gains. For each calorimeter crystal, energy deposition and coordinate of energy deposition are recorded. A first estimate of the energy is based on upon the calorimeter: an energy "centroid" is computed, as well as energy "moments". These variables are described next. 


\section{Energy Variables for the LAT}

The variable known as CTBBestEnergy is the default energy for analysis with the Fermi-LAT; its value is given by one of the following variables for reconstructed energy: EvtEnergyCorr, CalLkHdEnergy, or CalCfpEnergy. The reconstructed energy variable called EvtEnergyCorr is given as the sum of corrected energy in the tracker and corrected energy in the calorimeter. Studies show that it provides better performance in terms of resolution at low energies. CalCfpEnergy is the energy reported by the full profile fit, using the Tkr direction if provided, otherwise using the Cal direction.

\subsection{LAT Performance}

\subsubsection{Instrument Response Functions}

The instrument response functions (IRFs) for the LAT are parametrizations of effective area, point spread function, and energy resolution. The P6_V1 instrument response function, developed before launch, was the result of Monte Carlo simulations, and validations with beam-test runs and surface muons [23]. The first LAT catalog, and the analysis in this dissertation, were done assuming P6_V3_DIFFUSE instrument response functions. This IRF is a flight PSF, an improvement over the pre-launch performance estimates such as P6_V1, in that it takes into account pile-up events and accidental coincidences causing ghost signals[24]. The instrument performance depends, to a certain extent, on the IRFs being applied.

Cuts applied to select the P6_V3_DIFFUSE photon sample are the following:

ObfGamStatus $>0$ \&\& CTBCORE $>0.1$ \&\& CTBBestEnergyProb $>0.1$ \&\& CTBBestEnergy $>10$ \&\& CTBBestEnergyRatio<5 \&\& CTBClassLevel $>2$

A classification tree model, trained on beam-test and Monte Carlo simulation, is used by the Fermi-LAT collaboration to separate photons and furthermore, distinguish them into classes. CTBCORE is a classification tree (CT) based variable which assigns 
a probability that the direction solution is correct. CTBBestEnergyProb is a CTassigned probability of an event being photon, between 0 and 1 . CTBClassLevel $>2$ selects diffuse class events, with an integer value of this variable of 3. CTBBestEnergyRatio $=$ CTBBestEnergy $/ \max (1.0$, CalEnergyRaw $)$; the cut on this variable cuts out events with too little energy in the calorimeter. ObfGamStatus is a 32-bit status word output from Gamma, Heavy Ion, Minimum-Ionizing, Diagnostic Filters, as calculated by the Onboard Filter simulator.

\subsubsection{Point Spread Function of the LAT}

The point spread function (PSF) refers to the probability distribution of reconstructed direction of incident $\gamma$-rays from a point source. It is a measure of angular resolution for a point source. The PSF of the LAT is generally a function of the incident photon's energy and inclination angle, as well as event selection. By design considerations, particularly of the tracker, the Fermi-LAT PSF is 3 times better than EGRET's above $1 \mathrm{GeV}$.

It has been parametrized analytically as the following function, known as the King function:

$$
(1-1 / \gamma)\left[1+\frac{1}{2} \frac{u}{\gamma}\right]^{-\gamma}
$$

where $u=\frac{1}{2}\left(\frac{r}{\sigma}\right)^{2}$. The exponential shape parameter is given by $\gamma \simeq 2$, and the scale factor $\sigma$ corresponds to the rms for Gaussian distribution (in the case of small $u)$. The scale factor has been parametrized by the following function:

$$
\sigma(E)=\sqrt{\left[c_{0}\left(\frac{E}{100}\right)^{-0.8}\right]^{2}+c_{1}^{2}}
$$

with $\left(c_{0}, c_{1}\right)=(0.058,0.000377)$ for front converting events and $(0.096,0.0013)$ for back converting events [25].

Of interest here is the PSF that can be computed from the Monte Carlo (MC) generated sample of low energy gamma-rays. It can be computed from $68 \%$ containment of the difference between true direction (as given by the $\mathrm{MC}$ value) and 

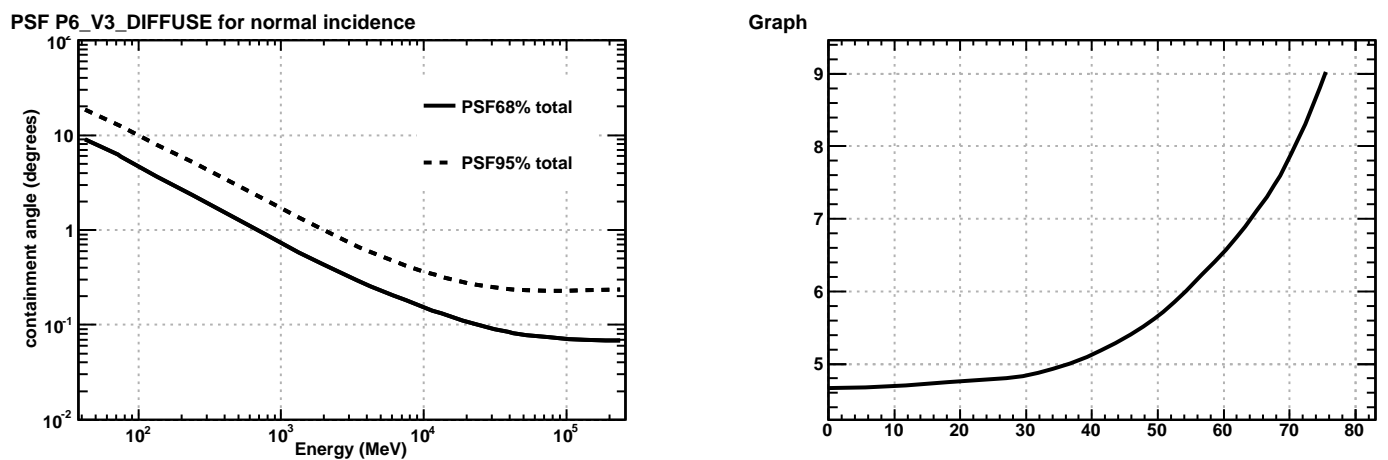

Figure 2.9: Left: PSF versus energy for normally incident photons. The PSF for front converting events is narrower than that of back-converting events at all energies. The 95\% containment PSF is larger than the $68 \%$ containment PSF, as expected. Right: at $100 \mathrm{MeV}$ gamma-ray energy, the dependence on the incidence angle is plotted; it is clear that that for larger off-axis angles, the PSF becomes larger.

reconstructed direction in the LAT. It decreases as a function of energy, from about $3.5^{\circ}$ at $100 \mathrm{MeV}$ to $0.8^{\circ}$ at $1 \mathrm{GeV}$, as shown in Fig. 2.9.

The PSF at low energy is dominated by multiple scattering of $e^{+}$and $e^{-}$. This predicts that the PSF should have a $1 / E$ dependence with energy $E$. The bremsstrahlung process also affects the PSF.

\subsubsection{Energy Resolution}

Energy resolution in the LAT, denoted $\frac{\Delta E}{E}$, for $68 \%$ containment has been studied by the Fermi-LAT collaboration. This function generally decreases from $50 \mathrm{MeV}$ to 1 GeV as shown in Fig. 2.10 26]. The energy measurement in the range below $200 \mathrm{MeV}$ is problematic. While front converting events have a smaller angular resolution than back converting events, they also have a larger energy resolution. In this dissertation, front and back converting events are considered.

\subsubsection{Effective Area}

The effective area of the LAT below $200 \mathrm{MeV}$ is relatively low, compared to the values at higher energy, as shown in Fig. 2.11. The peak of the effective area us achieved near 


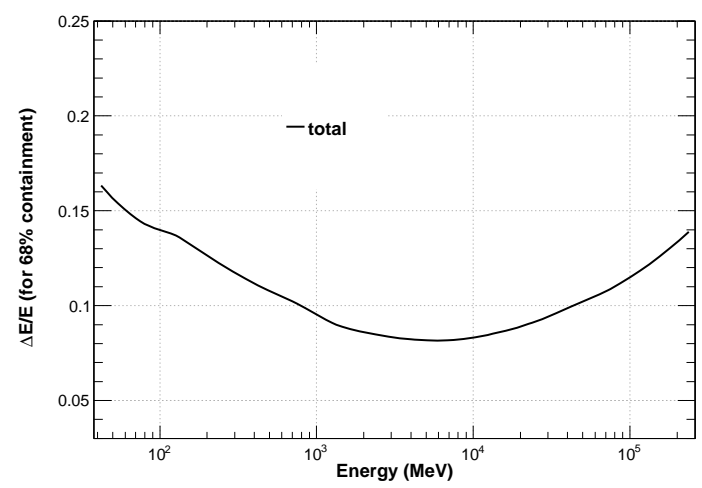

Figure 2.10: Energy resolution curve for the P6_V3 IRF, including front and back sections of the tracker. Below $1 \mathrm{GeV}$, however, the energy resolution increases as a function of energy.
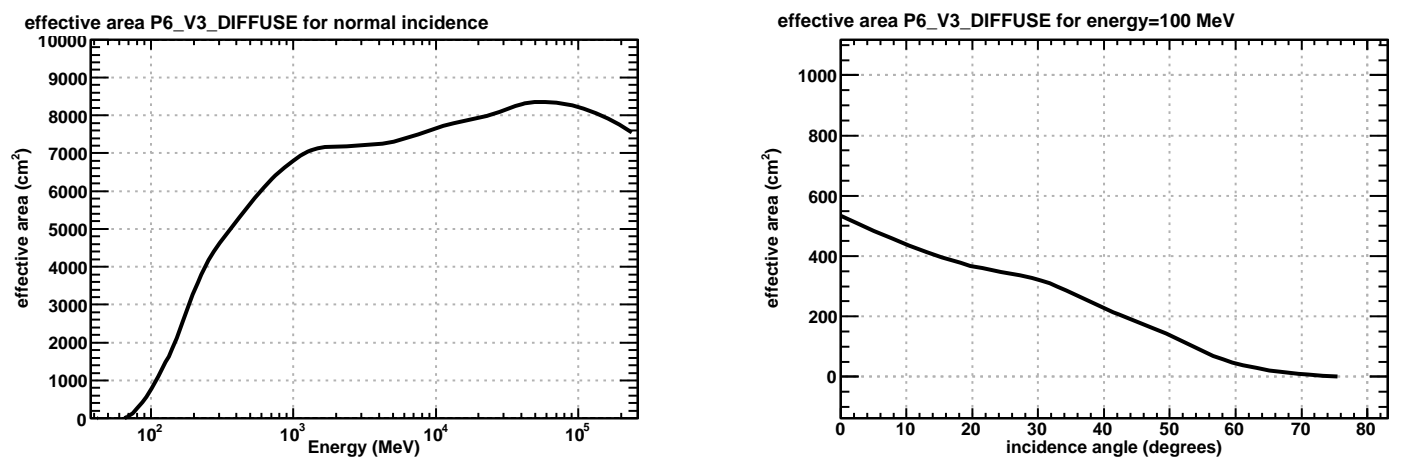

Figure 2.11: Left: Effective area $\left(\mathrm{cm}^{2}\right)$ versus gamma-ray energy $(\mathrm{MeV})$, for normally incident photons. Right: Effective area at $100 \mathrm{MeV}$, as a function of incidence angle. 


\begin{tabular}{lcc}
\hline specification & Fermi-LAT & EGRET \\
\hline PSF $\left(^{\circ}\right), 68 \%$ containment at $100 \mathrm{MeV}$ & 4.7 & 5.6 \\
Energy Resolution $(\%)$ at $100 \mathrm{MeV}$ & 14 & 11 \\
Effective Area $\left(\mathrm{cm}^{2}\right)$ at $100 \mathrm{MeV}$ & 550 & 1000 \\
Flux Sensitivity $\left(\mathrm{cm}^{-2} \mathrm{~s}^{-1}\right)$ & $6 \times 10^{-9}$ & $1.3 \times 10^{-7}$ \\
\hline
\end{tabular}

Table 2.1: Comparison of performance specifications of Fermi-LAT and EGRET, as relevant for the gamma-ray energies considered in this paper [23, 27]. The flux sensitivity is evaluated for a high-latitude point source with a $E^{-2}$ spectrum, with 1 year of data, for $E>100 \mathrm{MeV}$.

$3 \mathrm{GeV}$ [23]. This affects the systematic uncertainties in the energy range considered in this dissertation, approximately $10 \%$. The effective area of the diffuse class is lower than the effective area for the transient class; for P6_V3_DIFFUSE IRFs, the decrease is around $50 \%$. In using the diffuse class events, which is appropriate for faint sources, the effective area is reduced. In addition, the further off-axis the events, the less the effective area of the LAT.

\subsubsection{Comparison with EGRET}

One of the reasons this analysis sets better gamma-ray limits than HR did is due to the improvements in many specifications in comparison with the EGRET. Although EGRET had a better energy resolution at $100 \mathrm{MeV}$, PSF, effective area, and flux sensitivity were better in Fermi-LAT. A comparison of the specifications at $100 \mathrm{MeV}$ are shown in Table 2.1 . 


\section{Chapter 3}

\section{Obtaining the Differential Flux from KK Graviton Decay}

The theory of KK graviton emission in nucleon-nucleon gravi-bremsstrahlung occurring within supernova cores lies within the framework of the ADD model of Large Extra Dimensions. In supernova cores, with number densities of nucleons approaching $0.16 \mathrm{fm}^{-3}$, nucleon-nucleon interactions are dominant. The originators of the LED model, as well as other investigators, assumed virtual pion exchange as the mechanism for nucleon-nucleon gravi-bremsstrahlung. Nucleon-nucleon gravi-bremsstrahlung is a strong force interaction, and pion exchange was assumed since pions mediate the nuclear force over longer distances. However, this overlooks some of the nuclear physics of the problem. Subsequently, Hanhart, Savage and others developed a theory of nucleon-nucleon gravi-bremsstrahlung as a nucleon-nucleon scattering process 7]. Although a full treatment would require using the nuclear scattering $t$-matrix, they invoked low-energy theorems for nucleon-nucleon bremsstrahlung to simplify the calculation. They determined that the quantum amplitude for the process was related to nucleon-nucleon scattering data in the limit of soft radiation, i.e., graviton energy much lower than the energy of the scattering nucleons. Hannestad and Raffelt proposed the concept that such produced KK gravitons could be trapped in the gravitational potential of neutron stars from a progenitor supernova 28]. Essential points of the theory of Hannestad and Raffelt are described here, as relevant to the 


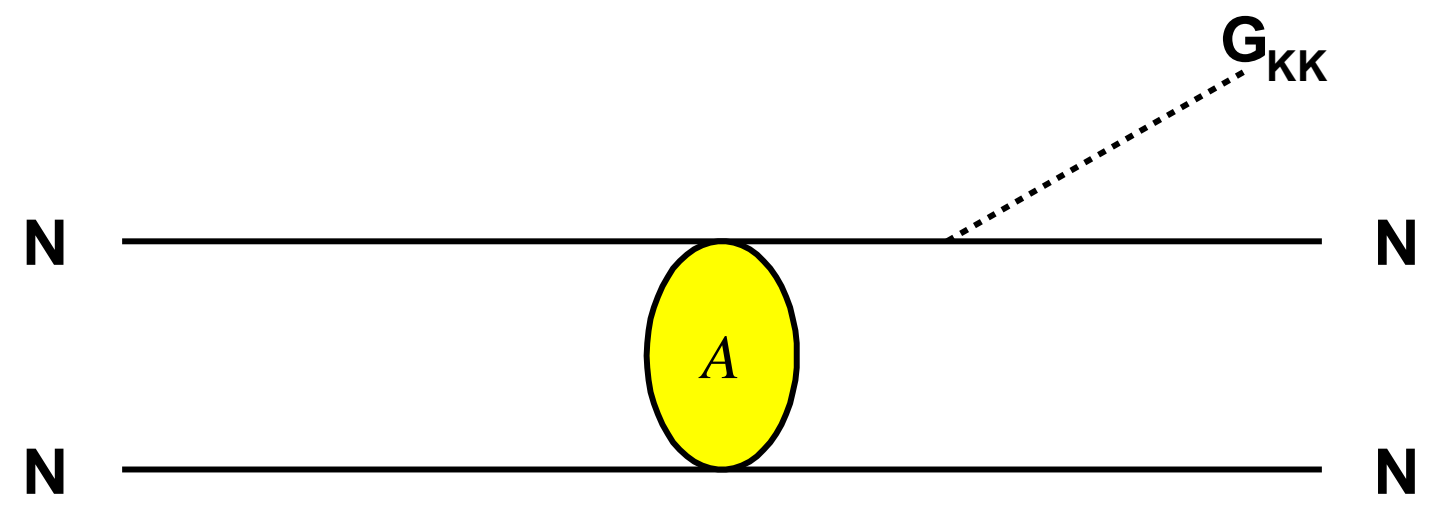

Figure 3.1: The Feynman diagram for KK graviton $\left(G_{K K}\right)$ emission, assuming a nucleon-nucleon scattering process. Nucleons are denoted by solid lines and the KKmodes $G_{K K}$ is denoted by dashed line. The solid oval containing an $A$ denotes an insertion of the full $N N$ scattering amplitude.

derivation of the spectral energy distribution (SED) of neutron stars.

\subsection{Emission of KK Gravitons by Nucleon-Nucleon Bremsstrahlung}

Nucleon-nucleon bremsstrahlung occurs during supernova core collapse, and KK gravitons are emitted. This involves a nucleon-nucleon scattering amplitude, as depicted in Figure 3.1. In the limit of soft radiation, where the energy of the emitted graviton is much less than the energy of the incoming nucleons, the actual details of the scattering process are not important.

According to theory, the energy loss rate into any type of radiation in the long wavelength limit, i.e., where the energy transfer $\omega$ is small (and so can be ignored), can be expressed as:

$$
Q=\int \frac{d^{3} \mathbf{k}}{2 \omega\left(2 \pi^{3}\right)} \omega S(-\omega)
$$


$S(-\omega)$ has been defined by Raffelt as the structure function for bremsstrahlung emission, while $S(\omega)$ denotes bremsstrahlung absorption, with the convention that negative sign refers to energy loss.

According to detailed balancing, the ratio of amplitudes between emission and absorption of a particle of energy $\omega$ is:

$$
\frac{S(-\omega)}{S(\omega)}=\exp (-\omega / T)
$$

where $T \simeq 30 \mathrm{MeV}$ is the presumed supernova core temperature [29], in units of energy $(\mathrm{MeV})$ 1. It thus follows that:

$$
S(-\omega)+S(\omega)=S(\omega)(1+\exp (-\omega / T))
$$

or

$$
S(-\omega)=\bar{S}(\omega) \frac{2}{1+e^{\omega / T}},
$$

with the definition

$$
\bar{S}(\omega)=\frac{1}{2}[S(\omega)+S(-\omega)]
$$

To leading order, according to equations (3.1) and (3.5), the differential energy-loss rate becomes:

$$
\frac{d Q}{d \omega}=\frac{\omega^{2}}{(2 \pi)^{2}} \bar{S}(\omega) \frac{2}{1+\exp (\omega / T)}
$$

with:

$$
\bar{S}(\omega)=\frac{S_{0}}{\omega^{2}}
$$

where:

$$
S_{0}=\frac{1024 \sqrt{\pi}}{5} \frac{G_{N} \sigma n_{B}^{2} T^{5 / 2}}{M^{1 / 2}} .
$$

In the previous equation, Newton's constant is $G_{N}=6.708 \times 10^{-33} \hbar c\left(\mathrm{MeV} / c^{2}\right)^{-2}, \sigma$ is the nucleon-nucleon scattering cross section of $25 \mathrm{mb}, n_{B}$ is the number density of baryons of $0.16 \mathrm{fm}^{-3}$, and $M$ is the isospin-averaged nucleon mass of $938 \mathrm{MeV} / c^{2}$,

\footnotetext{
${ }^{1}$ The conversion constant between temperature $(\mathrm{K})$ and energy corresponding to that temperature is Boltzmann's constant $k_{B}=8.62 \times 10^{-11} \mathrm{MeV} / \mathrm{K}$
} 
28]2. .

We are concerned with the square of the amplitude of the process, $|\mathcal{A}|^{2}$, and this is related in terms of the nucleon-nucleon differential scattering cross section:

$$
\frac{d \sigma}{d \Omega}=\frac{M^{2} \pi^{4}|\mathcal{A}|^{2}}{(2 \pi)^{6}} .
$$

Assuming that the emission of soft gravitons $(\omega \rightarrow 0)$ is a classical process, we obtain the usual flat bremsstrahlung process:

$$
\frac{d E_{g}}{d \omega}=\frac{32 G_{N}}{5 \pi} E_{i}^{2} \sin ^{2} \theta_{C M} .
$$

Then the differential energy-loss rate for a neutron medium in the long wavelength limit may be expressed as:

$$
\left.\frac{d Q}{d \omega}\right|_{\omega \rightarrow 0}=\int d \Gamma \mathcal{S}|\mathcal{A}|^{2} \frac{32 G_{N}}{5 \pi} E_{i}^{2} \sin ^{2} \theta_{C M},
$$

where $S=\frac{1}{4}$ represents a statistics factor for identical particles in the initial and final states, and $d \Gamma$ represents neutron phase-space integration. The following relation:

$$
\Gamma_{n n}=\int d \Gamma \mathcal{S}|\mathcal{A}|^{2}=\sigma n_{B}^{2}\left(\frac{4 T}{\pi M}\right)^{1 / 2},
$$

is used to integrate the differential energy-loss rate, which evaluates to:

$$
\left.\frac{d Q}{d \omega}\right|_{\omega \rightarrow 0}=\Gamma_{n n} \frac{128}{5 \pi} G_{N} T^{2}=\frac{256}{5 \pi^{3 / 2}} \frac{G_{N} \sigma n_{B}^{2} T^{5 / 2}}{M^{1 / 2}}=\frac{S_{0}}{(2 \pi)^{2}},
$$

representing the classical bremsstrahlung losses of the medium and relation to the previously defined constant $S_{0}$. The sum over KK-graviton modes, in the limit of continuously spaced KK modes, becomes:

$$
\Sigma_{m} \rightarrow \Omega_{n} R^{n} \int d m m^{n-1},
$$

\footnotetext{
${ }^{2}$ The integrated cross-section $\sigma$ is determined as $25 \mathrm{mb}$ as related to experimental proton-proton scattering data corrected for Coulomb effects [7], assuming non-degenerate nuclear matter.
} 
where $\Omega_{n}$ is the surface of the $n$-dimensional unit sphere, given in terms of the Gamma function as:

$$
\Omega_{n}=\frac{2 \pi^{n / 2}}{\Gamma(n / 2)}
$$

In addition, by relativistic kinematics, $k$ can be expressed in terms of $\omega$ and $m$ as $\sqrt{\omega^{2}-m^{2}}$. Thus the emission rate, per unit time per unit volume, as expressed in (3.1) should be written as a double integral over $m$ and $\omega$. Furthermore, the quantum field theory calculations by Hanhart, Reddy and Savage have shown that for a fixed KK graviton mode of mass $m$, the energy loss rate should be multiplied by the following factor [7]:

$$
\left(\frac{19}{18}+\frac{11}{9} \frac{m^{2}}{\omega^{2}}+\frac{2}{9} \frac{m^{4}}{\omega^{4}}\right)
$$

Combining the contributions to the graviton emission rate from equations (3.1), (3.16), and (3.14), we obtain as the energy loss rate into gravitons as

$$
\begin{aligned}
Q_{n} & =\frac{\Omega_{n} R^{n}}{(2 \pi)^{2}} \int_{0}^{\infty} d \omega S(-\omega) \omega^{n+1} \\
& \times \int_{0}^{\omega} d m\left(\frac{m}{\omega}\right)^{n-1} \sqrt{1-\left(\frac{m}{\omega}\right)^{2}}\left(\frac{19}{18}+\frac{11}{9}\left(\frac{m}{\omega}\right)^{2}+\frac{2}{9}\left(\frac{m}{\omega}\right)^{4}\right),
\end{aligned}
$$

which can be expressed in the notation:

$$
Q_{n}=\frac{\Omega_{n} R^{n}}{(2 \pi)^{2}} \int_{0}^{\infty} d \omega S(-\omega) \omega^{n+1} \int_{0}^{1} d \mu G_{n-1}(\mu),
$$

where $G_{p}(\mu)=\mu^{p} \sqrt{1-\mu^{2}}\left(\frac{19}{18}+\frac{11}{9} \mu^{2}+\frac{2}{9} \mu^{4}\right)$, and $\mu=m / \omega$. The previous equation may be written in the form:

$$
Q_{n}=g_{n} f_{n}(R T)^{n} Q_{0}
$$

with the following definitions: 


$$
\begin{gathered}
g_{n}=\Omega_{n} \int_{0}^{1} d \mu G_{n-1}(\mu), \\
f_{n}=\int_{0}^{\infty} d(\omega / T) \frac{(\omega / T)^{n+2}}{1+\exp (\omega / T)},
\end{gathered}
$$

$$
\begin{aligned}
Q_{0} & =\frac{512}{5 \pi^{3 / 2}} \frac{G_{N} \sigma n_{B}^{2} T^{7 / 2}}{M^{1 / 2}} \\
& =1.467 \times 10^{22} \mathrm{MeV} \mathrm{cm}{ }^{-3} \mathrm{~s}^{-1}(T / 30 \mathrm{MeV})^{7 / 2}\left(\rho / 3 \times 10^{14} \mathrm{~g} \mathrm{~cm}^{-3}\right)^{2}\left(f_{K K} / 0.01\right) .
\end{aligned}
$$

$f_{K K} \simeq 0.01$ is the estimated fraction of core-collapse energy radiated away as $G_{K K}[30,31]$.

\subsubsection{KK Gravitons in the Gravitational Potential of Proto- Neutron Star}

Assuming a Newtonian model for the gravitational potential of the neutron star, the potential per unit mass is:

$$
U(r)= \begin{cases}-\frac{G_{\mathrm{N}} M_{\mathrm{NS}}}{R_{\mathrm{NS}}}\left(\frac{3}{2}-\frac{1}{2} r^{2}\right), & r<1 \\ -\frac{G_{\mathrm{N}} M_{\mathrm{NS}}}{R_{N S}}, & r \geq 1 .\end{cases}
$$

where $r$ is the radial distance from the neutron star center, scaled to the neutron star radius ( $r=0$ at the NS center, $r=1$ at the surface), $U_{N S}=-G_{N} M_{N S} / R_{N S}=$ -0.159 , and $M_{N S}=1.4 M_{\odot}$ is the NS mass and $R_{N S}=13.0 \mathrm{~km}$ is the NS radius. The value of the neutron star radius is determined simplistically from assuming a constant density of $3 \times 10^{14} \mathrm{~g} / \mathrm{cm}^{3}$ for the nuclear matter in the neutron sta1 3 .

In the case of trapping of the gravitons produced in the supernova core, whose volume is defined by the proto-neutron star, $g_{n}$ may be replaced by $g_{n}^{(t r a p)}$.

\footnotetext{
${ }^{3}$ The constants such as density, mass, and radius are representative values chosen by Hannestad and Raffelt, which do vary between actual neutron stars. In this work we use the Hannestad and Raffelt values
} 


\begin{tabular}{|l|l|l|l|l|l|l|}
\hline$n$ & $f_{n}$ & $g_{n, H R}$ & $g_{n, H R}^{(t r a p)}$ & $f_{\text {trap }, H R}$ & $g_{n}^{(t r a p)}$ & $f_{\text {trap }}$ \\
\hline 2 & 1.80309 & 3.34106 & 0.606646 & 0.1816 & 0.919906 & 0.2753 \\
\hline 3 & 5.6822 & 4.28368 & 1.11663 & 0.2607 & 1.63925 & 0.3827 \\
\hline 4 & 23.3309 & 4.83907 & 1.61701 & 0.3342 & 2.30237 & 0.4758 \\
\hline 5 & 118.266 & 4.95239 & 1.99101 & 0.4020 & 2.75452 & 0.5562 \\
\hline 6 & 714.668 & 4.67306 & 2.16975 & 0.4643 & 2.92192 & 0.6253 \\
\hline 7 & 5021.01 & 4.11508 & 2.14449 & 0.5211 & 2.81594 & 0.6843 \\
\hline
\end{tabular}

Table 3.1: $n$-dependent constants, computed from integrals, related to the energy loss rate and number emissivity of KK gravitons.

$$
g_{n}^{(\text {trap })}=3 \int_{0}^{1} r^{2} d r \int_{1+U(r)}^{1} G_{n-1}(\mu) d \mu
$$

To calculate $g_{n}^{(t r a p)}$, one integrates over all accessible $\mu$, averaged over the volume of neutron star. Values for are tabulated in Table 3.1.1.

Here it is assumed, as was done by Hannestad and Raffelt, that the gravitons are on radial orbits. The lower bound on $\mu=1+U(r)$ arises from the relation that a $h$ remains trapped if the sum of kinetic and potential energy is less than zero, namely, $U(r)+\frac{1}{2} v^{2}<0$, where $v=\sqrt{1-\mu^{2}} 4$. Values of $g_{n}^{(\text {trap })}$ differ from those in the paper because they cited an incorrect numerical constant of $U_{N S}=-0.1139$.

\subsection{Theoretical Model}

Following HR, we start with the differential distribution of $G_{K K}$ created during the proto-neutron star core collapse with total energy $\omega$ and mass $m$ :

$$
\frac{\mathrm{d}^{2} N_{\mathrm{KK}, \mathrm{n}}}{\mathrm{d} \omega \mathrm{d} \mu}=\frac{\mathrm{d}^{2} Q_{n}}{\omega \mathrm{d} \omega \mathrm{d} \mu} \Delta t_{N S} V_{N S},
$$

where $\Delta t_{N S} \simeq 7.5 \mathrm{~s}$ is the time-scale for emission of $G_{K K}$ during the core collapse, and $V_{N S}=\frac{4}{3} \pi R_{N S}^{3}$ is the volume of the proto-neutron star (and current neutron star)

$$
{ }^{4} \mu^{2}>2[1+U(r)] \text { implies } \mu \geq 1+U(r)
$$


of radius $R_{N S} \simeq 13 \mathrm{~km}$. According to $\mathrm{HR}$, we have:

$$
\frac{\mathrm{d}^{2} Q_{n}}{\mathrm{~d} \omega \mathrm{d} \mu}=Q_{0}(R T)^{n} \Omega_{n} G_{n-1}(\mu) F_{n}\left(\frac{\omega}{T}\right),
$$

where $R$ is the extra dimension size, as in equation (1), $T \gtrsim 30 \mathrm{MeV}$ is the supernova core temperature, and $\Omega_{n}=2 \pi^{n / 2} / \Gamma(n / 2)$ is the surface of the $n$-dimensional unit hypersphere, with $\Gamma(\ldots)$ as the Gamma function. We also have,

In equation (3.29), the following functions are defined, where $q$ is an integer:

$$
\begin{aligned}
G_{q}(\mu) & =\mu^{q} \sqrt{1-\mu^{2}}\left(\frac{19}{18}+\frac{11}{9} \mu^{2}+\frac{2}{9} \mu^{4}\right) \\
F_{q}(\omega / T) & =\frac{(\omega / T)^{q}}{1+\exp (\omega / T)}
\end{aligned}
$$

Finally, the integral for the case of trapped KK gravitons, which make up the initial cloud bound to the NS, is given by:

$$
N_{K K, n}(t=0)=3 \int_{0}^{\infty} d \omega \int_{0}^{1} r^{2} d r \int_{1+U(r)}^{1} d \mu \frac{\mathrm{d}^{2} N_{K K, n}}{\mathrm{~d} \omega \mathrm{d} \mu} .
$$

In equation (3.32), HR assume that the graviton creation is isotropic at the dimensionless radial distance from the neutron star center, $r$, scaled to the neutron star radius, $R_{N S}$. The integration over $r$ is performed from the proto-neutron star's center to its surface, where $r=1$, and the condition $\mu>1+U(r)$ selects the $G_{K K}$ that are gravitationally trapped.

Since the $G_{K K}$ decay, at time $t$ from the core-collapse event, we have:

$$
N_{K K, n}(t)=N_{K K, n}(t=0) \exp \left(-\frac{\mu t}{\tau(m)}\right) .
$$

Then, the time derivative (absolute value), of equation (3.28), is given by: 


$$
\begin{aligned}
\left|\frac{\mathrm{d} \dot{N}_{K K, n}}{\mathrm{~d} \mu \mathrm{d} \omega}\right| & =\kappa \frac{m^{4}}{\omega} N_{K K, n}(t) \\
& =Q_{0}(R T)^{n} \Omega_{n} \Delta t_{N S} V_{N S} \kappa T^{2} G_{n+3}(\mu) F_{n+2}(\omega / T) \exp \left(-\frac{\mu t}{\tau(m)}\right) .
\end{aligned}
$$

\subsubsection{Determining the differential flux by Monte Carlo Sim- ulation}

We determine the differential flux according to a Monte Carlo simulation that uses equation (3.34). The 7 steps taken in the simulation are given below:

1. Sample $\omega$ from $F_{n+2}(\omega / T)$, as in equation (3.34), for $0<\omega / T<20$ (setting $T=30 \mathrm{MeV})$. For $\omega / T>20$, there is negligible contribution from the integral of $F_{n+2}(\omega / T)$, for the $n$ considered.

2. Sample $\mu$ from $G_{n+3}(\mu)$, as in equation (3.34), between $\mu_{\min }$ and $\mu_{\max }$. (Note that the sampling steps $1 \& 2$ are independent of each other, see HR.) To simplify the orbit calculation with only a small error, we assume that all of the created $G_{K K}$ start their orbit at the center of the NS $(r=0) 5$. Thus we have $\mu_{\min }=0.807$, corresponding to the $G_{K K}$ escape velocity, and $\mu_{\max }=0.926$, corresponding to the minimum velocity to reach the neutron star surface, from $r=0$. Having determined a value of $\mu$, we determine the $G_{K K}$ Lorentz factor $\gamma=1 / \mu$ and initial velocity $\beta=\sqrt{1-\mu^{2}}$. Given the geometry of the SN explosion, we assume, as do HR, that the $G_{K K}$ orbits are radial. Using $\omega$ and $\mu$, from steps 1 and 2, we determine a value for the mass, $m=\mu \omega$. Representative distributions of $m$, for different values of $n$, are shown in Figure 3.2 .

3. Determine whether the $G_{K K}$ decays into gamma-rays at the present time.

Since we know the mass at this point, and given the age of the neutron star, $t_{\text {age }}$, we calculate the exponential decay fraction, $F_{\text {decay }}=\exp \left(-\frac{\mu t_{\text {age }}}{\tau(m)}\right)$. We

\footnotetext{
${ }^{5}$ We have calculated that this approximation is in the direction of making our limits more conservative.
} 


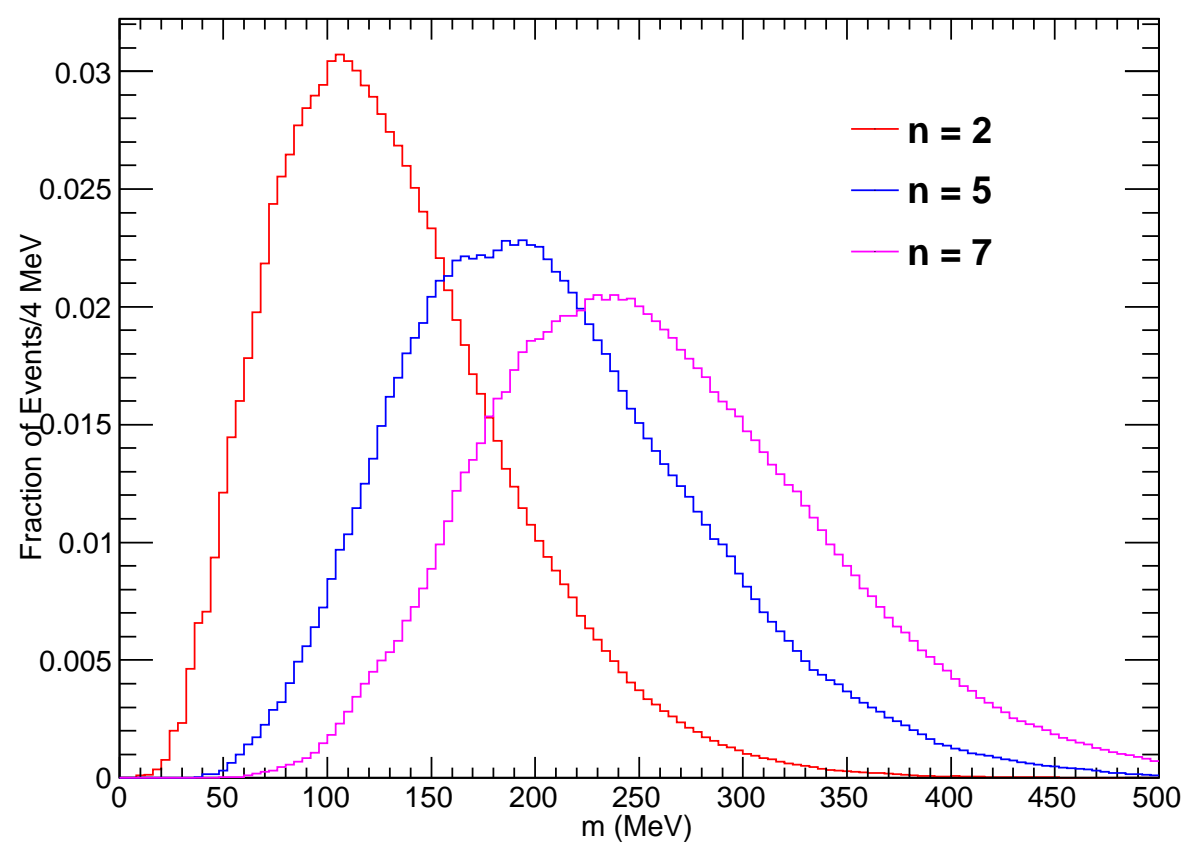

Figure 3.2: Unit-normalized distributions of KK graviton masses for $n=2,5,7$, as determined according to Monte Carlo simulation. 
sample a real number $u$ uniformly in the interval $[0,1]$ : if $u>F_{\text {decay }}$, then the event is rejected.

4. Sample the decay vertex $r_{0}$ for a given $\mu$. We sample $r_{0}$ for $0<r_{0}<r_{\max }$ ( $r_{\max }$ is defined in equation (3.36), but reject $r_{0}$ if it is less than 1 , due to the opacity of the neutron star matter to gamma-rays. This is according to $P(r ; \mu)$, a probability density function for $r$, given a value of $\mu$; examples are shown in Figure 3.3. In our model, due to the $G_{K K}$ emission radially outward during the SN core collapse, the $G_{K K}$ are not given any initial angular momentum; thus we assume that the $G_{K K}$ oscillate on radial paths (completely eccentric orbits) through the center of the neutron star. In spherical coordinates $(r, \theta, \phi)$, this is equivalent to the following constraints: $\dot{\theta}=\dot{\phi}=0$. The orbital radial distribution, $P(r ; \mu)$, is defined outside the neutron star by the radial Kepler equation, in which time is given as a function of the radial coordinate $r$ [32]

$$
t(r)=t_{k}\left(\arcsin (\sqrt{k r})-\sqrt{k r(1-k r)}+c_{1}\right)
$$

where

$$
k=r_{\max }^{-1}=\frac{1+1.5\left|U_{N S}\right|-\gamma}{\left|U_{N S}\right|}
$$

and

$$
t_{k}=\frac{R_{N S}}{\beta c k^{2}} \sqrt{\frac{k(1-k)}{1-\left|U_{N S}\right| / \beta^{2}}} .
$$

Inside the neutron star, the trajectory is given by the solution to

$$
\ddot{r}=-\left|U_{N S}\right| r
$$

Given the boundary conditions that $\left.\ddot{r}\right|_{r=0}=0$ and $\left.\dot{r}\right|_{r=0}=\beta c$, where $\beta$ is sampled as $\sqrt{1-\mu^{2}}$, the solution to the previous equation may be expressed as

\footnotetext{
${ }^{6}$ The radial Kepler equation is not manifestly periodic. However, the full orbit cycle includes an interval over $0<r<r_{\max }$, or a quarter of a cycle. Due to the symmetry of the orbit, our treatment of sampling the decay vertex from $t=0$ to $t\left(r_{\max }\right)$ is sufficient to obtain the full distribution of $r_{0}$.
} 


$$
r(t)=\frac{\beta}{\sqrt{\left|U_{N S}\right|}} \sin (\Omega t),
$$

where the parameter $\Omega=\sqrt{\left|U_{N S}\right|} c / R_{N S}=9.13 \times 10^{3} \mathrm{~s}^{-1}$.

Given $t=0$ is the time when the $G_{K K}$ starts from $r=0$, the radial distributions are determined by sampling time uniformly between the time when the $G_{K K}$ reaches the surface $t(r=1)$, and $t_{\max } \cdot t_{\max }$ is given by the time to achieve the maximum distance, $r_{\max }=1 / k . \quad c_{1}$ is determined from boundary conditions of position and velocity at the surface of the neutron star by solving the full equation of motion inside and outside the neutron star. The trajectories for a couple of values of $\beta$ are plotted in Figure 3.4, while the radial distributions for representative values of $\mu, P(r ; \mu)$, are shown in Figure 3.3 .

5. Sample the orbit direction isotropically $(-1<\cos \theta<1,-\pi<\phi<\pi)$. This selects an orbit direction:

$$
\hat{r}_{0}=\sin \theta \cos \phi \hat{x}+\sin \theta \sin \phi \hat{y}+\cos \theta \hat{z}
$$

where $\hat{x}, \hat{y}, \hat{z}$ are the unit coordinate directions in the NS frame. The $\hat{z}$ direction is chosen to align with the magnetic dipole axis of the NS. At the sampled decay vertex, $r_{0}$, we then obtain a velocity,

$$
\beta^{\prime}=R_{N S} \dot{r} / c
$$

where $\dot{r}=d r /\left.d t\right|_{r=r_{0}}$ is obtained numerically, and the Lorentz factor is:

$$
\gamma^{\prime}=1 / \sqrt{1-\beta^{\prime 2}}
$$

Using the determined values of $m$ and $\beta^{\prime}$ yields $p_{K K}^{\nu}$, the $G_{K K} 4$-momentum in the NS frame at the decay vertex. 


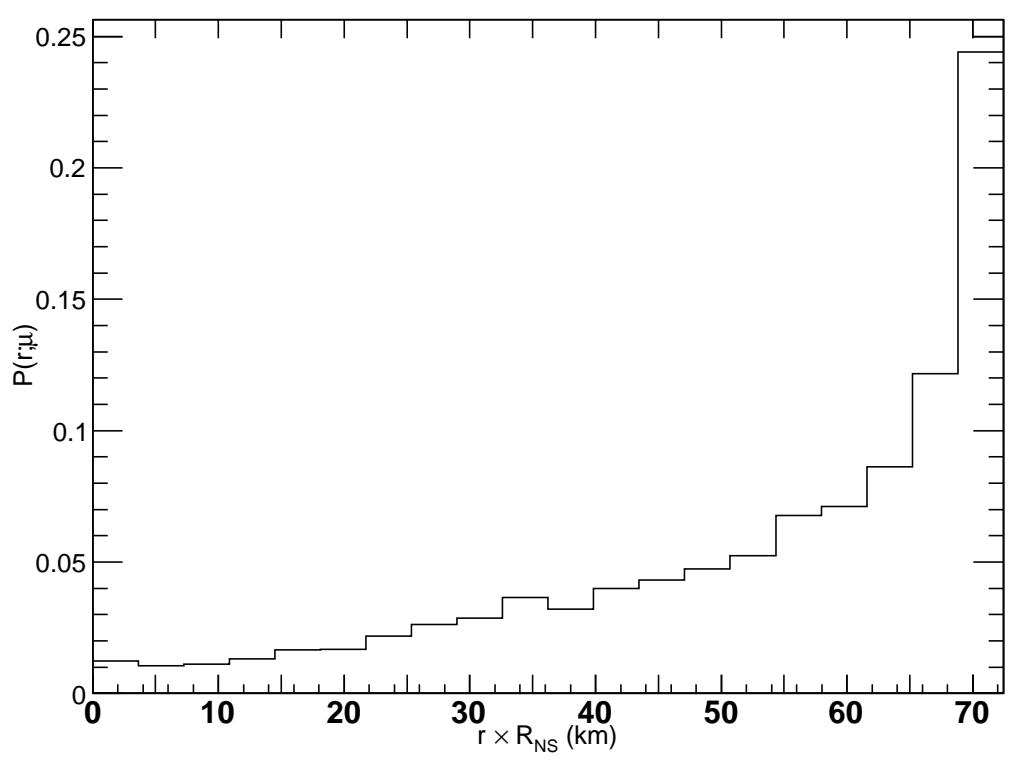

(a) $\mu=0.83$

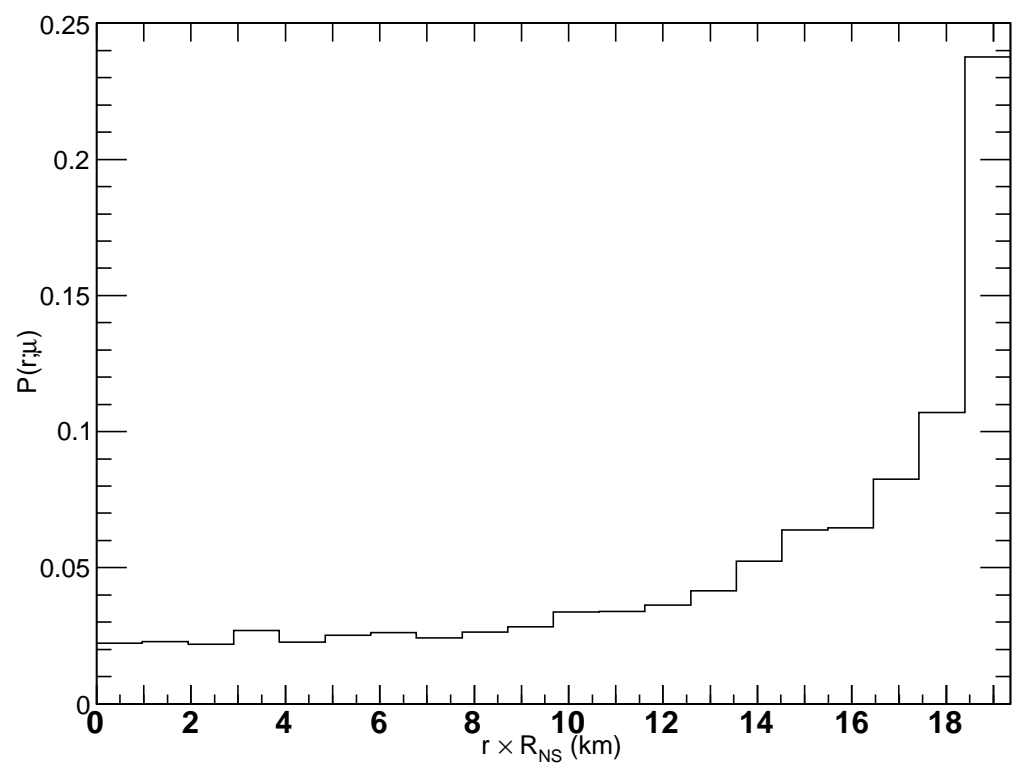

(b) $\mu=0.90$

Figure 3.3: Radial probability density functions, $P(r ; \mu)$, for 2 different values of $\mu$. There are 20 linearly-spaced bins over the interval $\left[0, r_{\max } \times R_{N S}\right]$, and the $y$-axis is the fraction of events per bin. 


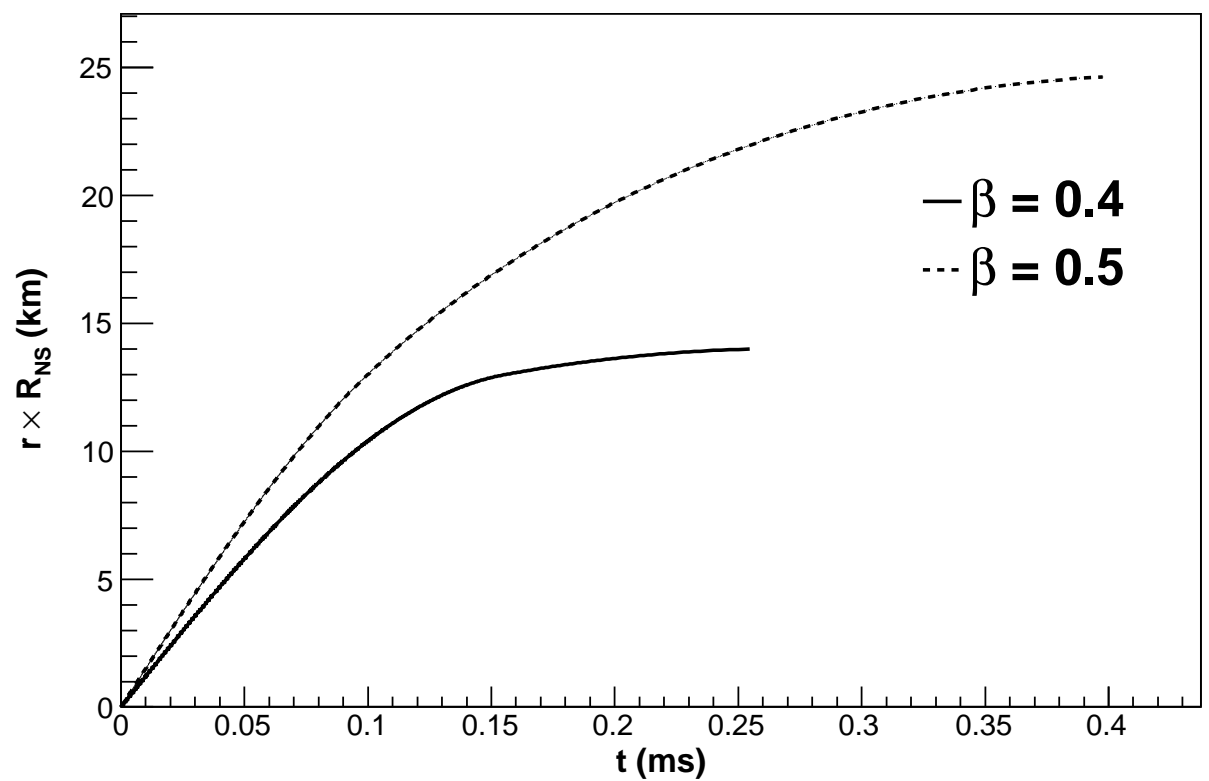

Figure 3.4: KK graviton trajectories for $\beta=0.4$ and $\beta=0.5$ (right). Notice that for larger $\beta$, the KK graviton may achieve a farther maximum distance, $r_{\max } \times R_{N S}$. This figure shows one quarter of the orbit cycle. 


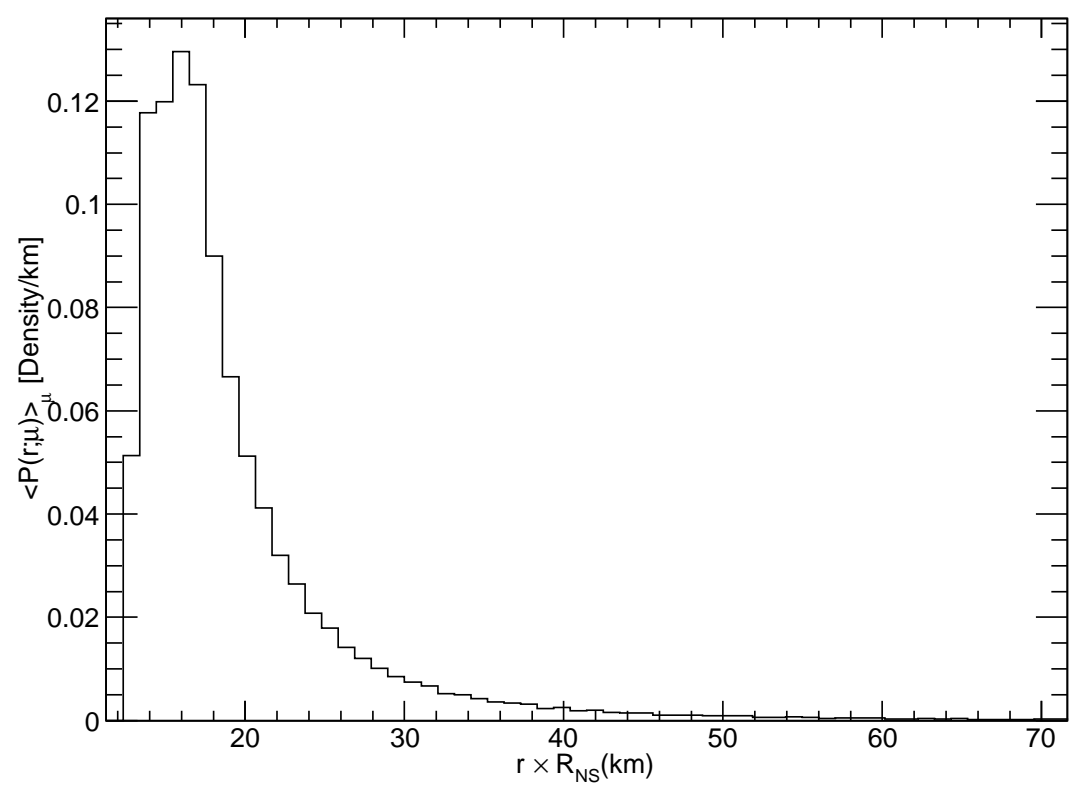

Figure 3.5: Unit-normalized radial distribution of decay vertices, as a function of radial coordinate, $r_{\mathrm{km}}$, for $n=2$. On the y-axis is plotted $<P(r ; \mu)>_{\mu}$, averaged over all $\mu$ between $\mu_{\min }$ and $\mu_{\max }$, for $1<r<5.5$. 


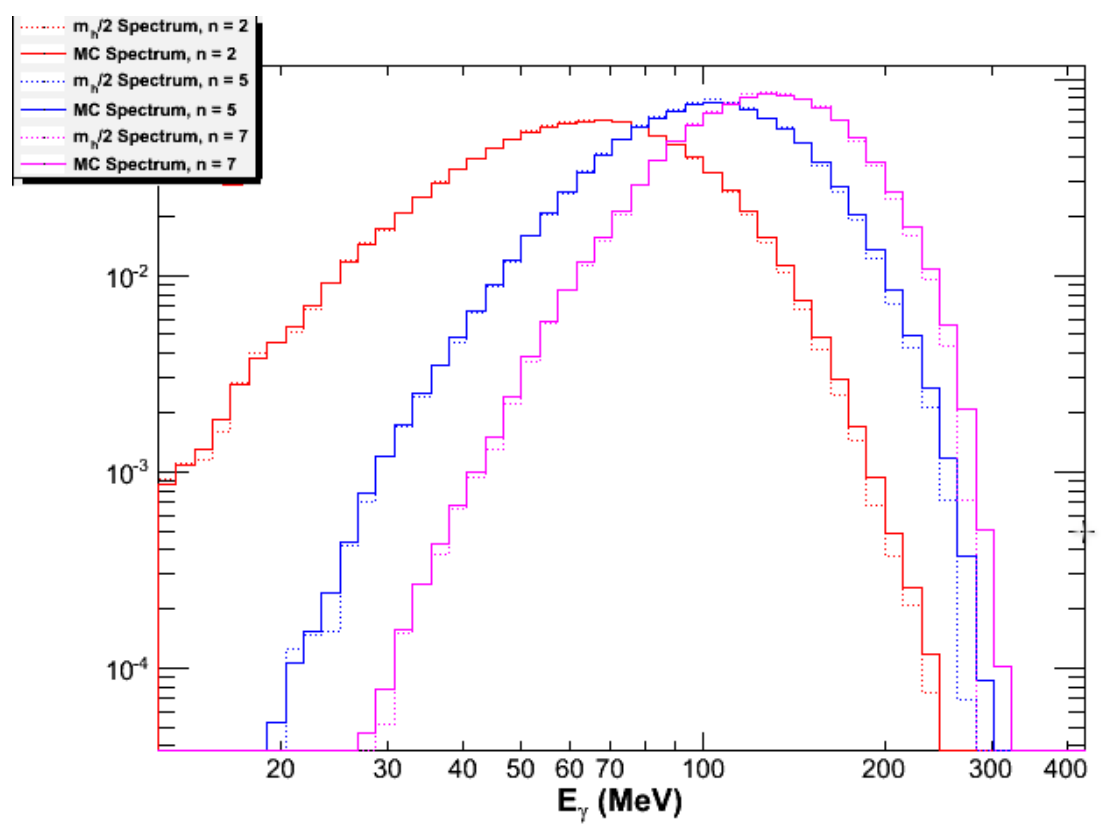

Figure 3.6: Unit-normalized gamma-ray energy distributions from graviton decay for $n=2,5,7$, in the case of no attenuation, assuming a relativistic distribution of gamma-ray energies, as computed according to Monte Carlo simulation. Nonrelativistic gamma-ray distributions, for which $E_{\gamma}=m / 2$, are shown for comparison as dashed histograms, as shown in the plot legend.

6. Determine the energy and momentum distribution of one of the two decay photons at decay point on the orbit with direction $\hat{r}_{0}$. We treat the other decay photon by multiplying the final flux by two. The energy is given by:

$$
E_{\gamma}=\frac{1}{2} \gamma^{\prime} m\left(1+\beta^{\prime} \cos \theta^{*}\right),
$$

with distributions for $n=2,5,7$ for the non-attenuated spectrum compared with the non-relativistic distributions of $m / 2$ plotted in Figure 3.6.

The components of the photon momentum vector are:

$$
\vec{p}_{\gamma}^{\prime}=p_{x^{\prime}, \gamma} \hat{x}^{\prime}+p_{y^{\prime}, \gamma} \hat{y}^{\prime}+p_{z^{\prime}, \gamma} \hat{z}^{\prime}
$$

in the neutron star frame relative to the direction of the $G_{K K}$, which are given 
by:

$$
\begin{aligned}
p_{x^{\prime}, \gamma} & =\frac{1}{2} m \sin \theta^{*} \cos \phi^{*} \\
p_{y^{\prime}, \gamma} & =\frac{1}{2} m \sin \theta^{*} \sin \phi^{*} \\
p_{z^{\prime}, \gamma} & =\frac{1}{2} \gamma^{\prime} m\left(\beta^{\prime}+\cos \theta^{*}\right) .
\end{aligned}
$$

The $z^{\prime}$ axis is defined by: $\hat{z}^{\prime}=\hat{r}_{0} . \theta^{*}$ is the polar angle between the direction of the $G_{K K}$ in the lab frame $\left(z^{\prime}\right)$ and the decay photon in the rest frame of the $G_{K K}$, and $\phi^{*}$ is the angle in the $\left(x^{\prime}-y^{\prime}\right)$ plane. In this frame, $\hat{x}^{\prime}$ is taken as perpendicular to $\hat{z}^{\prime}$ in the $z-z^{\prime}$ plane and in the direction of increasing $\theta$, and $\hat{y}^{\prime}=\hat{z}^{\prime} \times \hat{x}^{\prime}$.

We sample a $\cos \theta^{*}$ value uniformly over the interval [-1,1], and $\phi^{*}$ uniformly over the interval $[-\pi, \pi]$, given isotropic emission of photons in the rest frame of the gravitons. Subsequently, we obtain momentum components of the gammaray in the frame of the neutron star, $\vec{p}_{\gamma}$, by rotating back into the frame of the neutron star, using the direction of the momentum vector, $\vec{p}_{K K}$, as defined by step (5). This is needed for the next step. A schematic of the coordinate system is shown in Figure 3.7 .

7. Determine whether the photon pair-produces in the neutron star magnetosphere.

Approximations for the pair-production attenuation are according to the treatment in Refs. [33, 34]. The attenuation coefficient depends on the parameter:

$$
\chi\left(E_{\gamma}, \vec{p}_{\gamma}, \vec{r}\right)=\frac{E_{\gamma}}{m_{e} c^{2}} \frac{B_{\perp}\left(\vec{r}, \vec{p}_{\gamma}\right)}{B_{\mathrm{cr}}},
$$

where the critical field is given by

$$
B_{\mathrm{cr}}=\frac{m_{e}^{2} c^{3}}{e \hbar}=4.414 \times 10^{13} \mathrm{G}
$$

$B_{\perp}$ is the magnetic field component of the neutron star perpendicular to the 


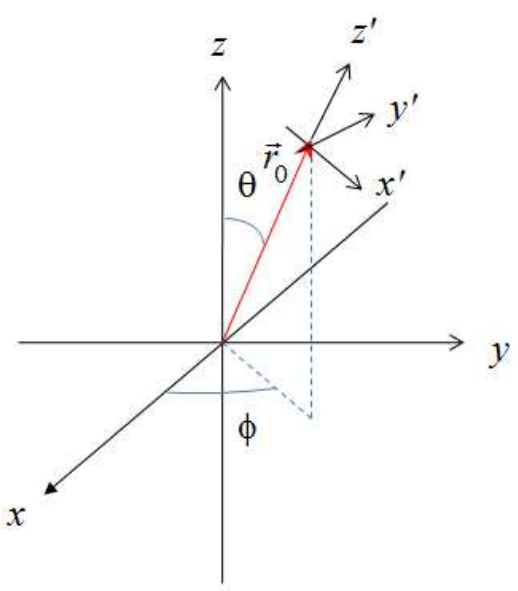

Figure 3.7: Coordinate system for $G_{K K}$ decay, as described in step (6). 
photon's momentum vector $\vec{p}_{\gamma}$, and $E_{\gamma}$ is the photon energy. For the magnetic field of the neutron star, we assume a static dipole field,

$$
\vec{B}(\vec{r})=\frac{3(\vec{m} \cdot \hat{r}) \hat{r}-\vec{m}}{r^{3}}
$$

with dipole moment $\vec{m}=\frac{1}{2} B_{\text {surf }} R_{N S}^{3} \hat{z}$. The attenuation coefficient, $\alpha$, is given by:

$$
\alpha\left(\chi\left(E_{\gamma}, \overrightarrow{p_{\gamma}}, \vec{r}\right)\right)=\frac{\alpha_{\mathrm{fs}}}{\lambda_{e}} \frac{B_{\perp}\left(\vec{r}, \vec{p}_{\gamma}\right)}{B_{\mathrm{cr}}} \alpha_{1}(\chi),
$$

where the reduced attenuation coefficient, $\alpha_{1}(\chi)$, is expressible as a function of $\chi$ in terms of a modified Bessel function of the second kind, with asymptotic limiting expressions for small and large values of $\chi$ (as plotted in Figure 3.8):

$$
\alpha_{1}(\chi)=0.16 \frac{1}{\chi} K_{1 / 3}^{2}\left(\frac{2}{3 \chi}\right)=\left\{\begin{array}{l}
0.377 e^{-\frac{4}{3 \chi}}, \chi \leq 0.1 \\
0.597 \chi^{-1 / 3}, \chi \geq 100
\end{array}\right.
$$

In equation (3.51), $\lambda_{e}=3.861 \times 10^{-11} \mathrm{~cm}$ is the reduced electron Compton wavelength and $\alpha_{\mathrm{fs}}$ is the fine structure constant. The asymptotic expressions are used in the Monte Carlo simulation, where appropriate, in order to save computer time.

The optical depth, $\tau_{p p}$, is calculated by path integrating the attenuation coefficient along the direction of the photon, from the point of decay, $\vec{r}_{0} \equiv<$ $x_{0}, y_{0}, z_{0}>$, out to where $r_{k m}=7 R_{N S}$ (where the field has attenuated to $0.3 \%$ of the surface field strength), according to

$$
\begin{aligned}
\tau_{p p} & =\int_{\text {path }} \alpha \mathrm{d} s \\
& =\int_{0}^{s_{\max }} \alpha\left(\chi\left(E_{\gamma}, \vec{p}_{\gamma}, \vec{r}_{0}+\hat{p}_{\gamma} s\right)\right) \mathrm{d} s
\end{aligned}
$$

In the preceding equation, $s_{\max }$, given by: 


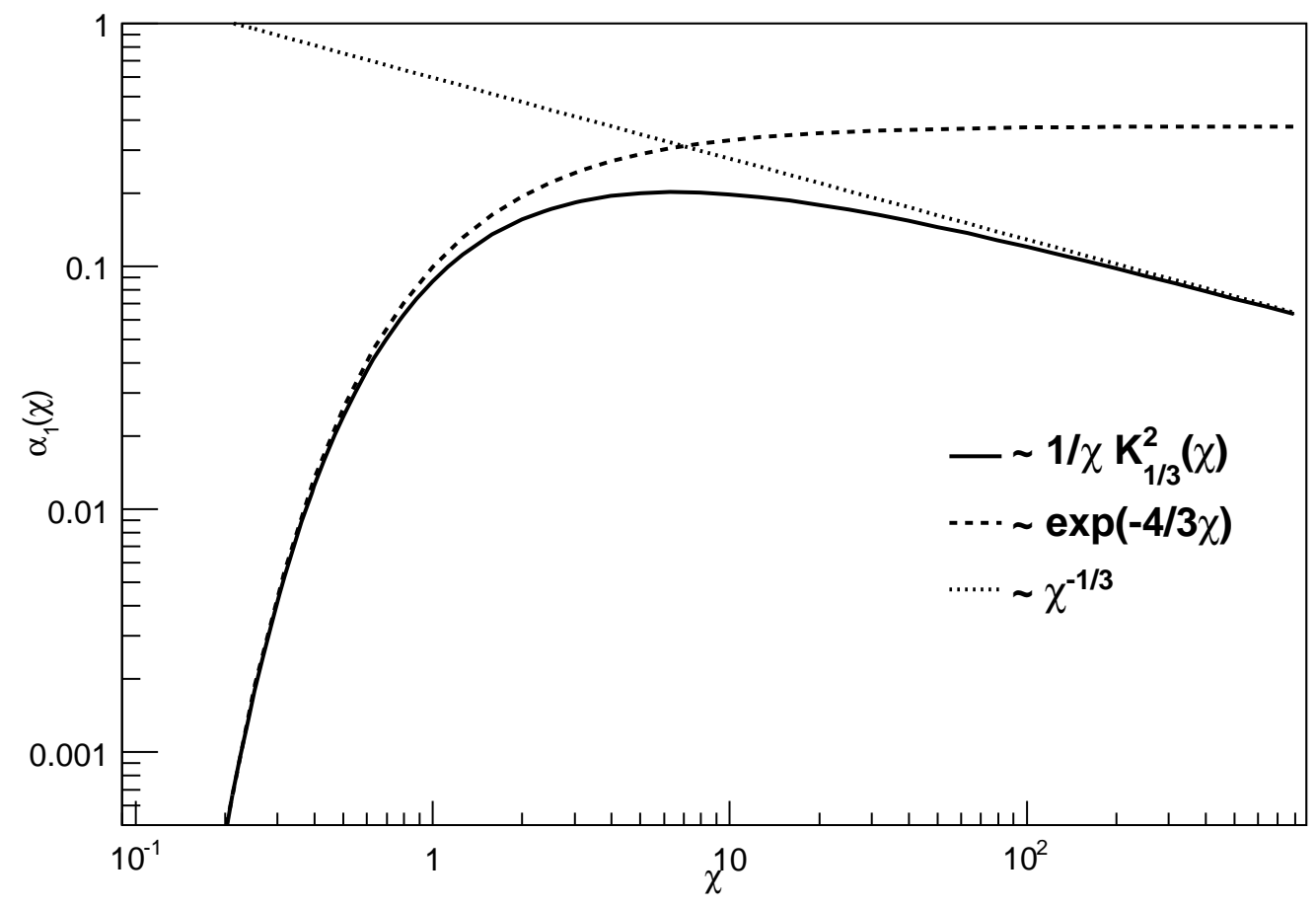

Figure 3.8: Plot of the reduced attenuation coefficient, $\alpha_{1}(\chi)$ and limiting asymptotic expressions corresponding to equation (3.52). 


\begin{tabular}{|l|l|l|}
\hline$n$ & $\bar{r}\left(R_{N S}\right)$ & $\bar{m}(\mathrm{MeV})$ \\
\hline 2 & 3.569 & 66.29 \\
\hline 3 & 3.451 & 78.72 \\
\hline 4 & 3.337 & 92.06 \\
\hline 5 & 3.227 & 105.2 \\
\hline 6 & 3.121 & 118.1 \\
\hline 7 & 3.019 & 130.7 \\
\hline
\end{tabular}

Table 3.2: Mean radial distances from neutron star centers for each $n$ in units of neutron star radii $\left(R_{N S}\right)$. Second column, mean graviton masses for $T=30 \mathrm{MeV}$.

$$
s_{\max }=-x_{0} p_{1}-y_{0} p_{2}-z_{0} p_{3}+\sqrt{\left(x_{0} p_{1}+y_{0} p_{2}+z_{0} p_{3}\right)^{2}+\left(7 R_{N S}\right)^{2}-x_{0}^{2}-y_{0}^{2}-z_{0}^{2}},
$$

refers to the path length where the photon with direction unit vector

$$
\hat{p}_{\gamma}=\frac{\vec{p}_{\gamma}}{\left|\vec{p}_{\gamma}\right|}=p_{1} \hat{x}+p_{2} \hat{y}+p_{3} \hat{z}
$$

is considered to have escaped the magnetosphere. The probability for photon survival from pair production in the Monte Carlo simulation is then taken as $P_{\mathrm{pp}}\left(E_{\gamma}, \vec{r}, \vec{p}_{\gamma}\right)=\exp \left(-\tau_{p p}\right)$. We sample a real number $v$ uniformly on the interval $[0,1]$ : if $v>P_{\mathrm{pp}}$, then the event is rejected.

\subsection{Differential Fluxes for the Various Sources}

We define a parameter, $\eta$, which quantifies the attenuation of the $G_{K K}$ decay signal, due to decay and pair-production:

$$
\eta \equiv \int_{100 \mathrm{MeV}}^{400 \mathrm{MeV}} \frac{d N_{n}}{d E_{\gamma}} \mathrm{d} E_{\gamma} /\left.\int_{100 \mathrm{MeV}}^{400 \mathrm{MeV}} \frac{d N_{n}}{d E_{\gamma}}\right|_{\text {non-atten }} \mathrm{d} E_{\gamma} .
$$

The attenuation parameters, $\eta$, for the various sources and different $n$, are shown in Table 3.3. The actual differential fluxes, with correction for pair production and 


\begin{tabular}{|l|l|l|l|l|l|l|}
\hline $\mathrm{n}$ & RXJ1856-3754 & J0108-1431 & J0953+0755 & J0630-2834 & J1136+1551 & J0826+2637 \\
\hline 2 & 0.335 & 0.031 & 0.221 & 0.359 & 0.309 & 0.332 \\
\hline 3 & 0.350 & 0.037 & 0.249 & 0.382 & 0.332 & 0.360 \\
\hline 4 & 0.361 & 0.041 & 0.276 & 0.402 & 0.351 & 0.385 \\
\hline 5 & 0.368 & 0.043 & 0.302 & 0.416 & 0.365 & 0.406 \\
\hline 6 & 0.370 & 0.042 & 0.325 & 0.424 & 0.374 & 0.419 \\
\hline 7 & 0.365 & 0.037 & 0.334 & 0.424 & 0.373 & 0.423 \\
\hline
\end{tabular}

Table 3.3: Table of values of the attenuation parameter $\eta$, defined by equation (3.56), for the different sources analyzed. These attenuation effects can be quite large. HR used only source RXJ1856-3754 and J0953+0755. These values are calculated for 100 $\mathrm{MeV} \leq E_{\gamma} \leq 400 \mathrm{MeV}$.

attenuation, are shown in Figures 3.9, 3.14. It may be observed that the expected SED from PSR J0108-1431 experiences greater degree of attenuation than the other sources considered, due to its greater age. Refer to Table 4.1 for parameters of the 6 sources considered in the analysis. 


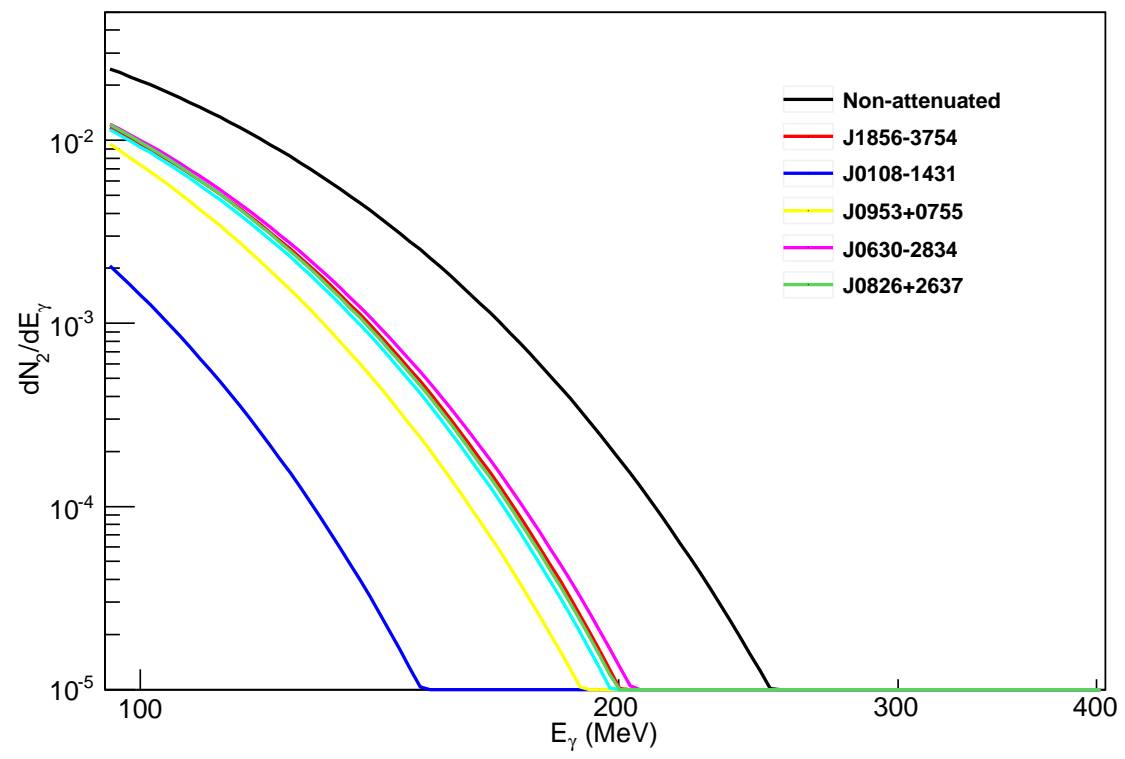

Figure 3.9: Differential fluxes for $n=2$ for the 6 sources and the non-attenuated spectrum.

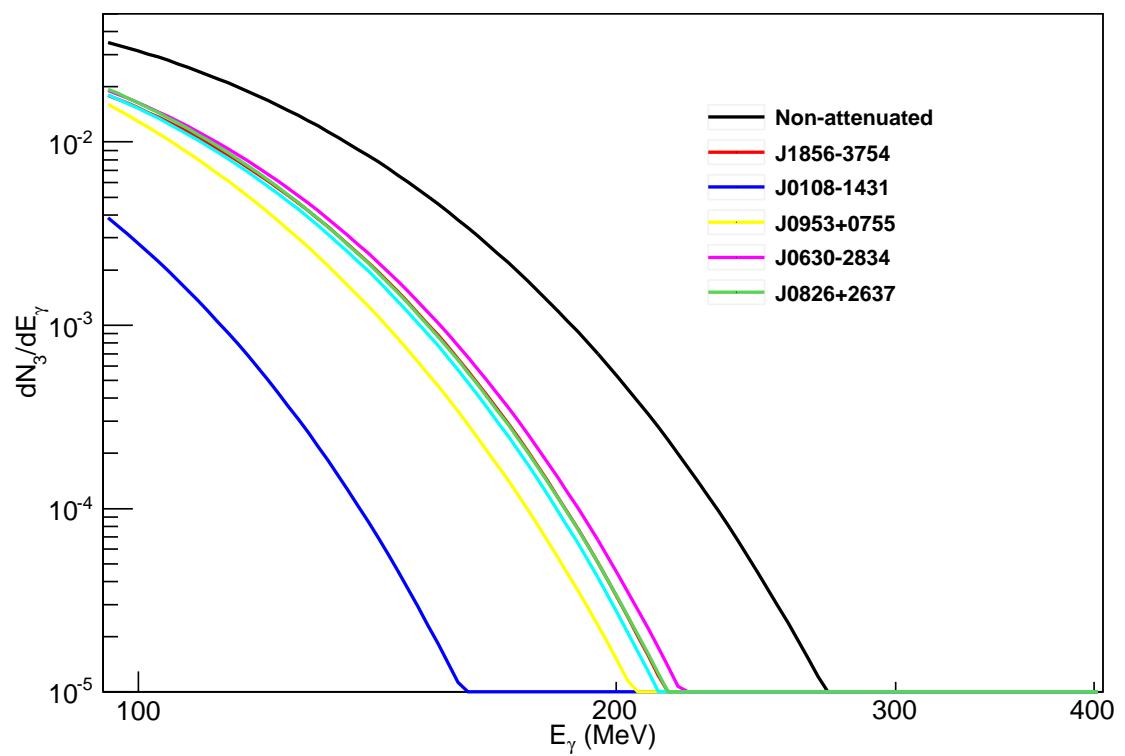

Figure 3.10: Differential fluxes for $n=3$ for the 6 sources and the non-attenuated spectrum. 


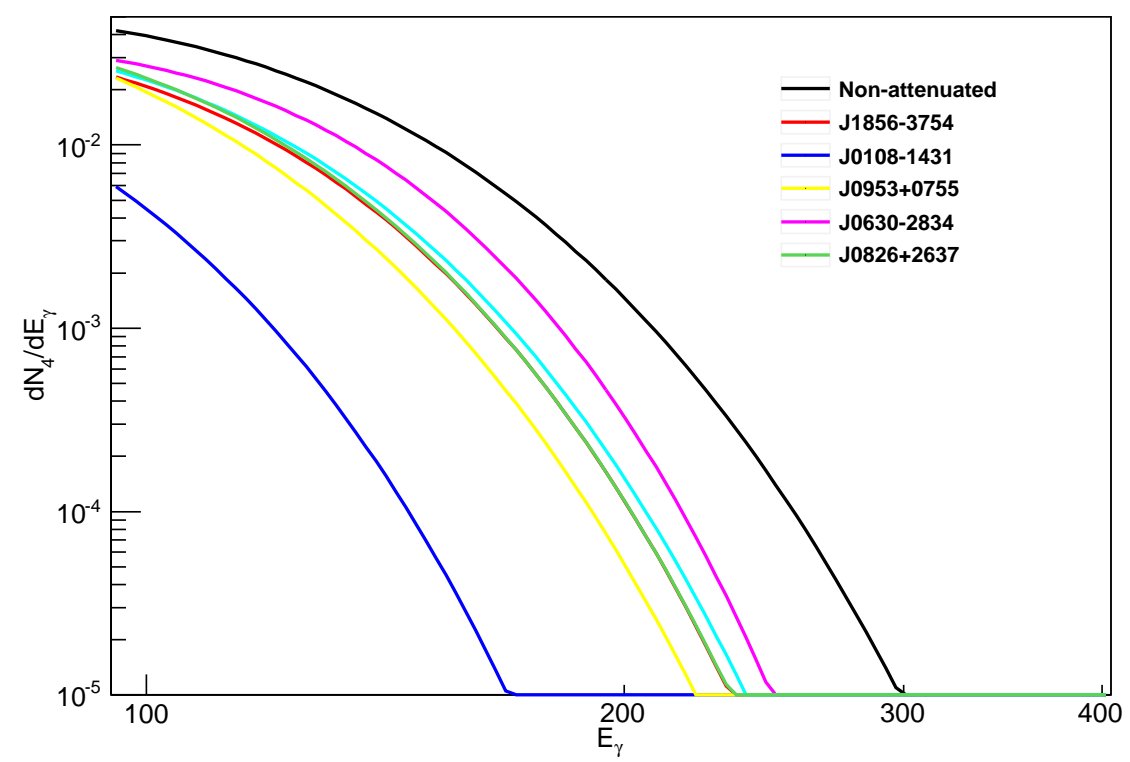

Figure 3.11: Differential fluxes for $n=4$ for the 6 sources and the non-attenuated spectrum.

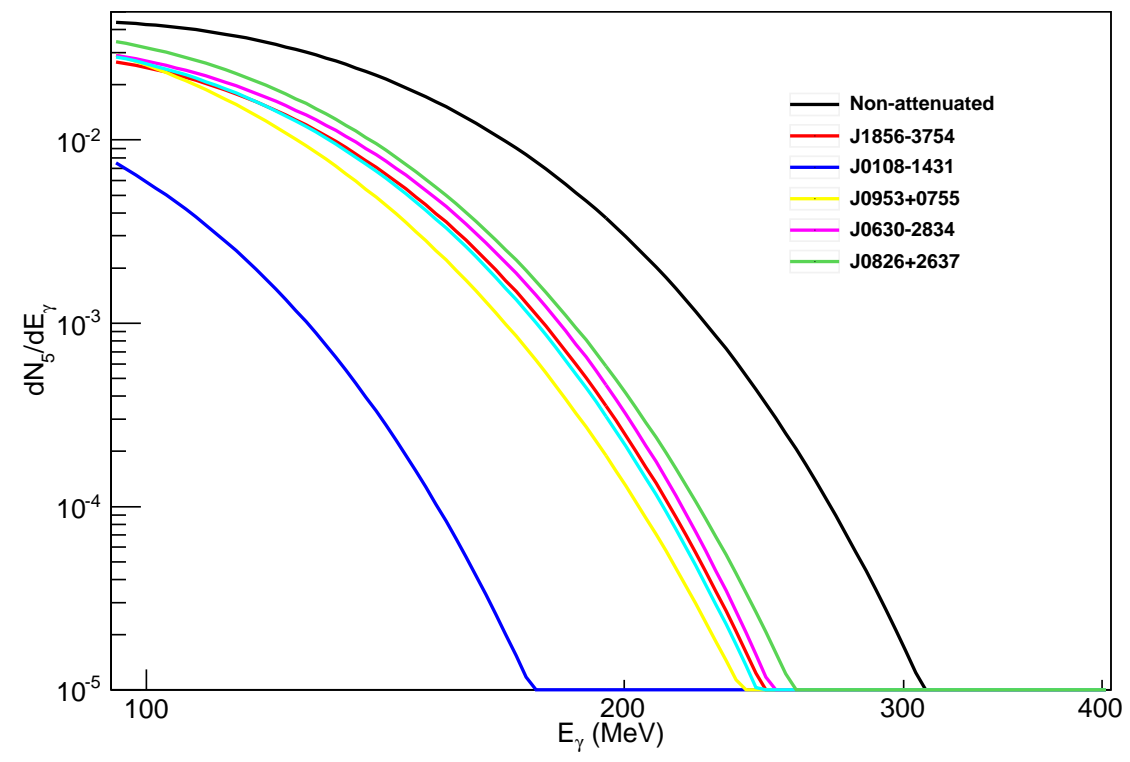

Figure 3.12: Differential fluxes for $n=5$ for the 6 sources and the non-attenuated spectrum. 


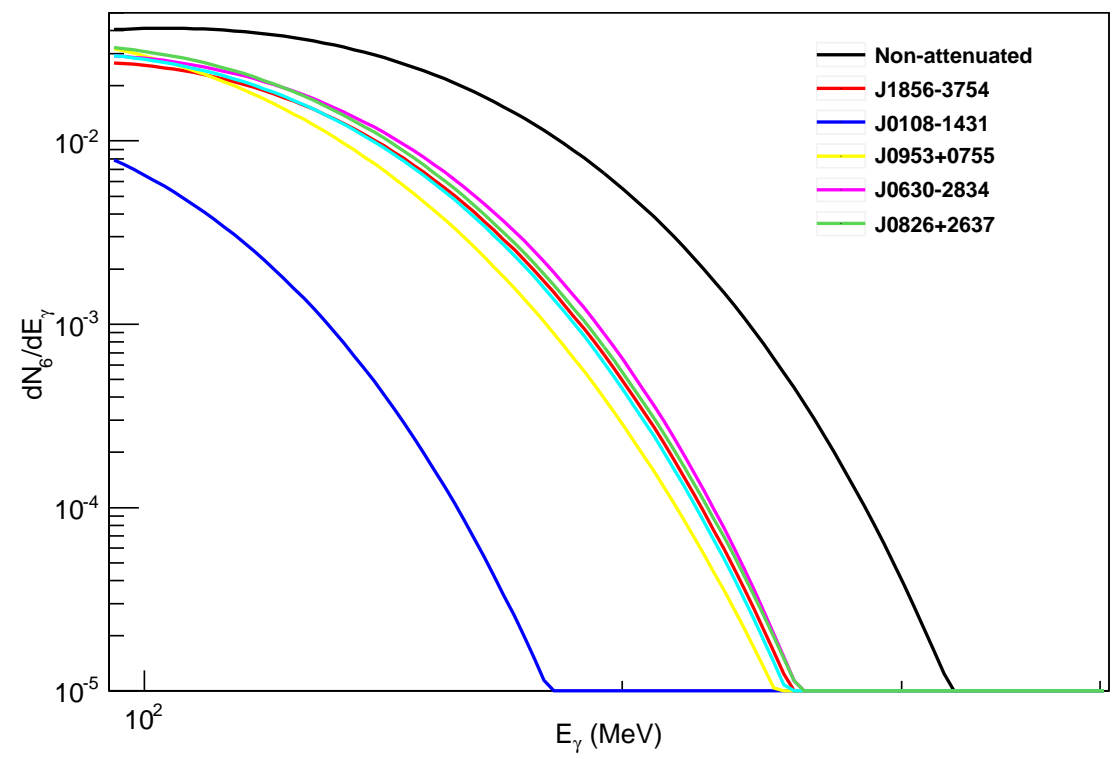

Figure 3.13: Differential fluxes for $n=6$ for the 6 sources and the non-attenuated spectrum.

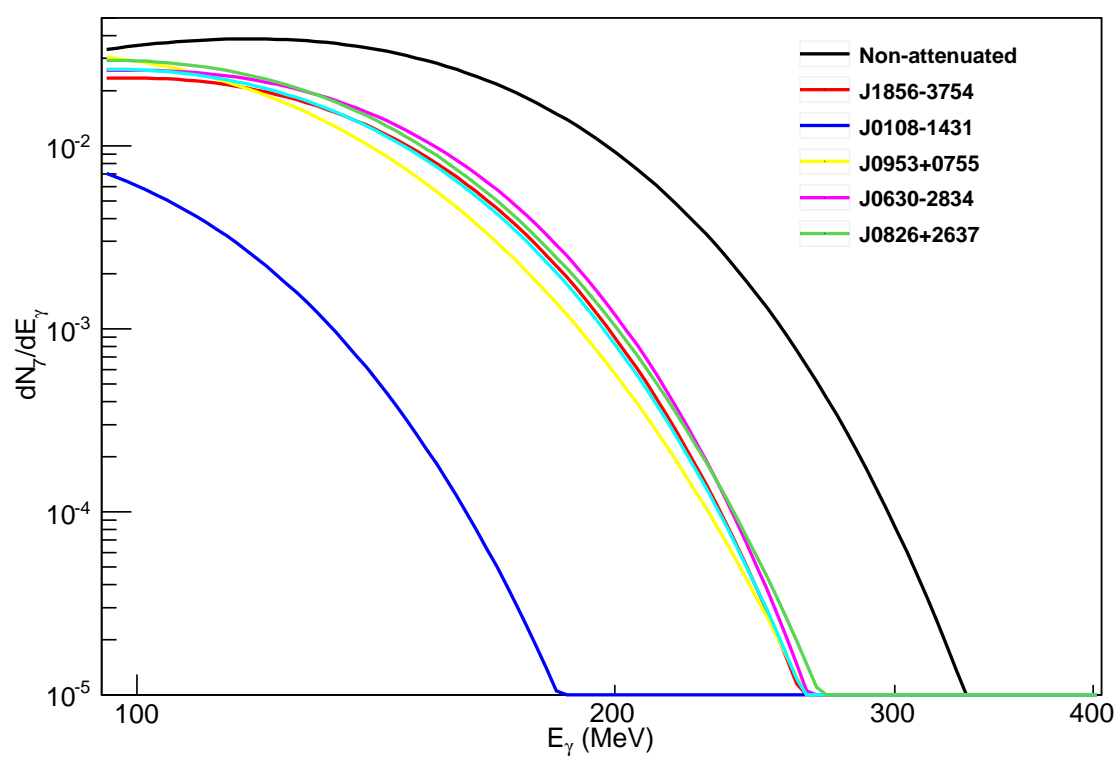

Figure 3.14: Differential fluxes for $n=7$ for the 6 sources and the non-attenuated spectrum. 


\section{Chapter 4}

\section{General Properties of Neutron Stars, and Properties of Sources Analyzed}

\subsection{Birth of Neutron Stars in Supernova Core Col- lapse}

Neutron stars are formed in the aftermath of a type II supernova core collapse. If the mass of the parent star is in the range $8 M_{\odot}<M<25 M_{\odot}$, a neutron star will be formed in the aftermath of the supernova core-collapse, with steps shown in Figure 4.2. Type II supernovae are usually found in the star-forming regions in the galactic spiral arms. The energy from released from gravitational contraction, i.e., the gravitational binding energy, is estimated as $3 \times 10^{53} \mathrm{erg}$, according to various sources of which the majority goes into neutrino decay channel during core collapse. It is estimated that the timescale of core collapse is given roughly by the diffusion time, $\tau_{\text {diff }}$, of neutrinos for performing a random walk, given by:

$$
\tau_{\text {diff }}=\frac{3 R^{2}}{l_{\nu} c},
$$




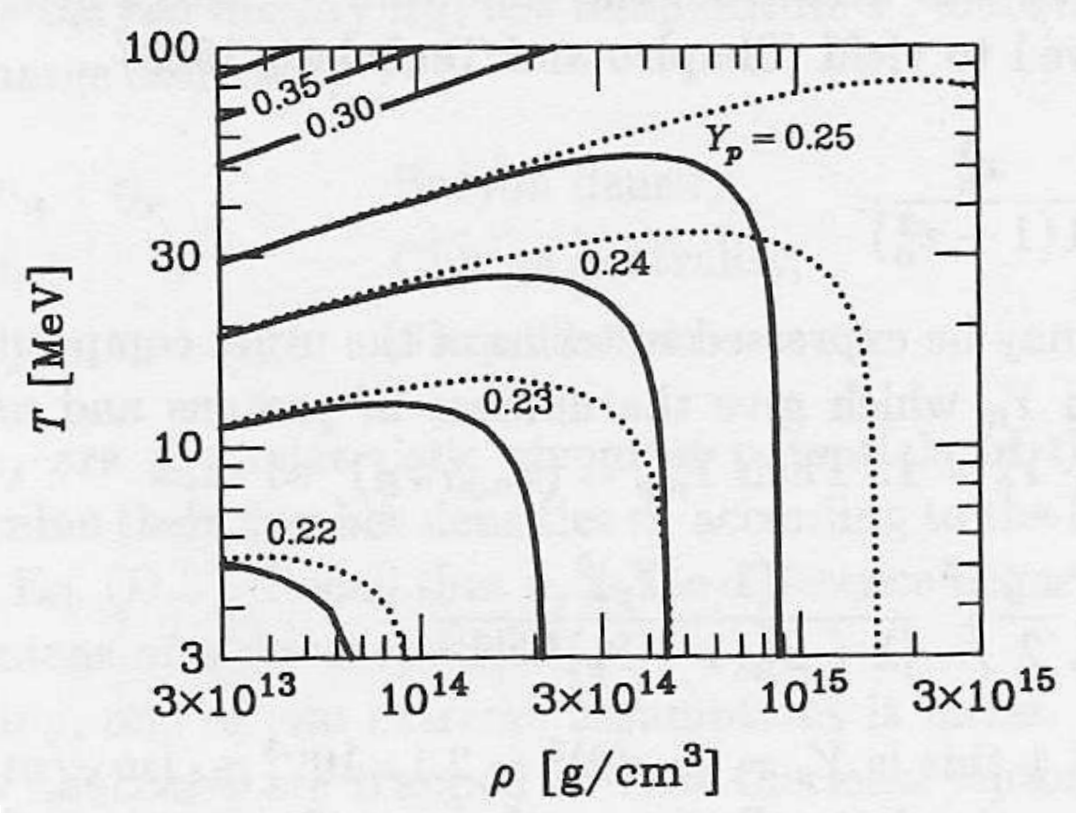

Figure 4.1: Allowed values of $\rho\left(\mathrm{g} \mathrm{cm}^{-3}\right)$ and $T(\mathrm{MeV})$. Contours represent different values of the proton fraction, $Y_{P}[29]$.

where $R$ is the stellar radius, and $l_{\nu} \simeq 0.5 \rho /\left(10^{12} \mathrm{~g} \mathrm{~cm}^{-3}\right)$ is the neutrino mean free path [35]. Estimates of the value of the timescale varies from $5 \mathrm{sec}$ to $7.5 \mathrm{sec}$, depending on the source; in this work, we use the value quoted by Hannestad and Raffelt, of $7.5 \mathrm{sec}$. The proto-neutron star generally has densities in excess of $10^{14} \mathrm{~g}$ $\mathrm{cm}^{-3}$. At these densities, nuclear repulsion due to the strong force is important. The temperature of the SN core is estimated to be $30 \mathrm{MeV}$ 29]. The temperature is related to the density of the medium, as well as the proton fraction, $Y_{P}$ of the medium, as shown in Figure 4.1] we take a reasonable value of $Y_{P}=0.24[29]$. From their inception, it is no surprise that neutron stars tend to be high velocity objects: the mean proper motion for pulsars younger than $3 \mathrm{Myr}$ is found by Hobbs to be $400 \pm 40 \mathrm{~km} \mathrm{~s}^{-1}$. Slight asymmetries in the explosion process are believed to be responsible for imparting the kick velocities [35]. 

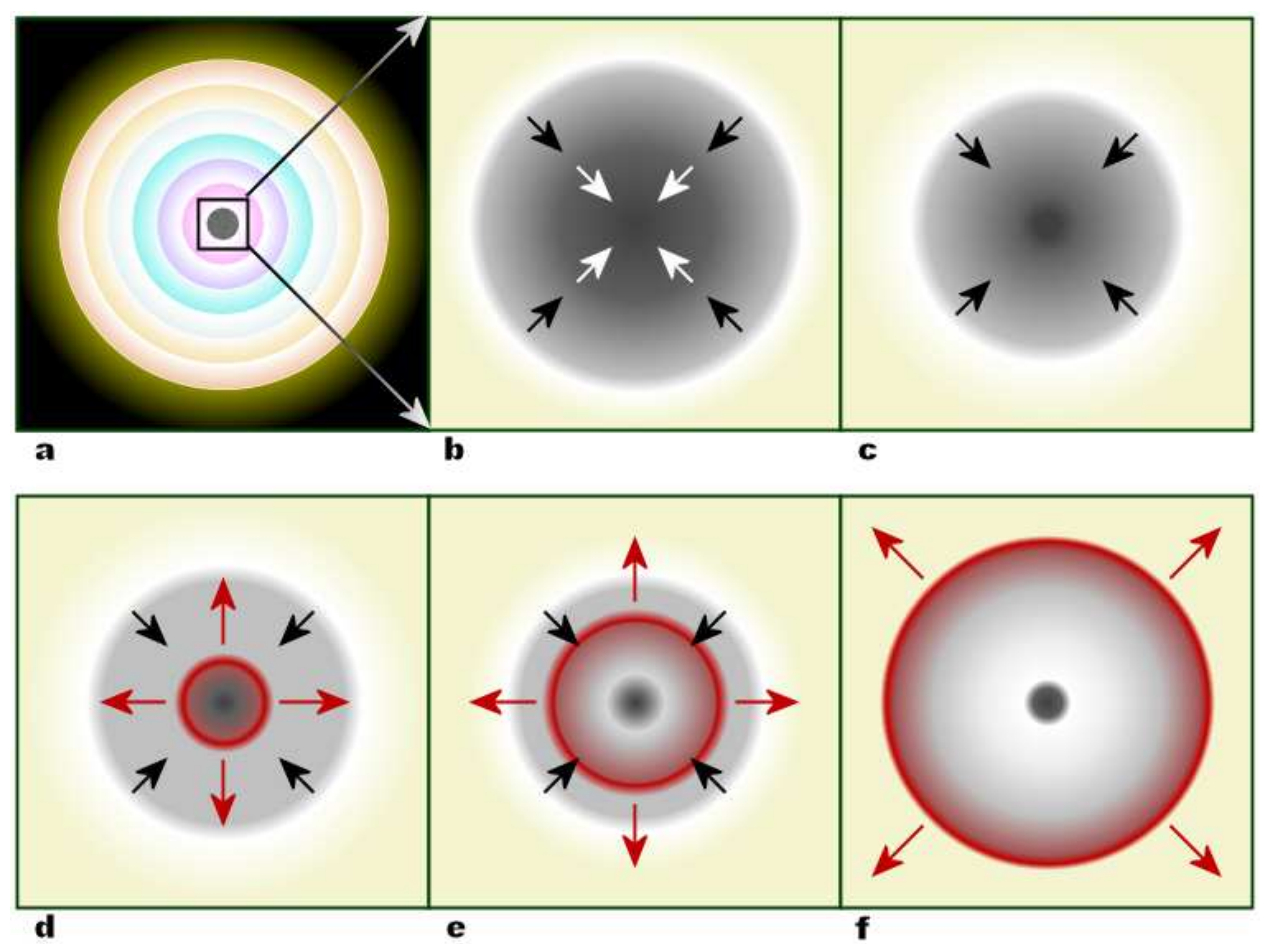

Figure 4.2: Schematic of the core-collapse event 36]. (a) At the end of the thermonuclear life of star, the core is composed of iron, and concentric shells outward contain successively lighter elements, such as silicon, oxygen, carbon, helium, and hydrogen. (b) Once the central iron core attains the Chandrasekhar mass of $M_{C h}=1.44 M_{\odot}$, electron degeneracy pressure no longer supports the iron core and it begins to contract.(c) The material in the core is compressed into neutrons. (d) A shock front proceeds away from the center (e) The shock stalls (f) but is re-energized by neutrinos from the newborn proto-neutron star. (e) Material is blasted away, and neutrinos carry away most of the energy. 


\subsection{Properties of Neutron Stars}

After neutrons were discovered by Sir James Chadwick in 1932, Walter Baade and Fritz Zwicky proposed the existence of the neutron star, in 1934. Neutron stars are compact stellar objects, created in the aftermath of a supernova explosion. They have radii on the order of $13 \mathrm{~km}$. They have strong magnetic fields, and they generally rotate with periods on the order of milliseconds to seconds. At the densities exhibited by neutron stars, general relativistic effects are important, and a space-time metric (such as the Swarzchild metric) is necessary for an accurate description of their properties such as mass, radius and gravitational field. The typical mass for a neutron star is given by the Chandrasekhar mass, which is $1.44 M_{\odot}$. Neutron stars are composed mostly of neutrons, and they are supported by neutron degeneracy pressure, since the fermions within the star obey the Pauli exclusion principle.

In general, the axis of rotation is different than the axis of magnetic dipole, and thus there is a beam of radiation, as shown in Figure 4.4. The first discovery of a radio pulsar occurred in 1967 by Bell and Antony Hewish, for which Hewish was awarded the 1974 Nobel Prize in Physics. Joseph Taylor and Russell Hulse were awarded the 1993 Nobel prize for discovering the first binary pulsar, PSR B1913+16, and verifying that their orbits decayed in accordance with the principles of general relativity and the theory of gravitational waves.

From a simple model of neutron stars, properties such as surface magnetic field and age can be inferred. The magnitude of the magnetic dipole moment for a neutron star is:

$$
|\vec{m}|=\frac{1}{2} B_{\text {surf }} R_{N S}^{3}
$$

The emission rate of energy, $\dot{E}$, due to non-alignment of the magnetic dipole axis with the rotation axis, at an angle $\alpha$, is given according Larmor's relation:

$$
\dot{E}=-\frac{2}{3 c^{3}}|\ddot{\vec{m}}|^{2}=-\frac{B^{2} R_{N S}^{6} \Omega^{4}}{6 c^{3}} \sin ^{2} \alpha .
$$

For a NS with moment of inertia $I$ and angular frequency $\Omega$, the rotational energy is 
given by:

$$
E=\frac{1}{2} I \Omega^{2}
$$

and hence the rate of loss of rotational energy can be expressed as:

$$
\dot{E}=I \Omega \dot{\Omega}
$$

If $P=2 \pi / \Omega$ is the period, the present age of the pulsar can be expressed as:

$$
\tau_{\text {age }} \simeq \frac{P}{2 \dot{P}} .
$$

The surface field for a magnetic dipole field pulsar is given by:

$$
B_{\text {surf }}=3.2 \times 10^{19} \mathrm{G} \sqrt{P \dot{P} / \mathrm{s}},
$$

where $\mathrm{G}$ is an abbreviation for gauss, the unit of magnetic induction ( 1 gauss $=10^{-4}$ Tesla).

The property of neutron stars which most affects our limits on LED is the distance from the Earth. In general, there are two methods for determining the distance to neutron stars which are also pulsars. One method is based on the parallax at Earth. The distance, $d$, in kpc, based on parallax, $\pi$, in mas, is given by the formula

$$
d=1 / \pi
$$

Pulsar parallaxes may be obtained from interferometry based on radio measurements with a long baseline, such as with the Long Baseline Array in Australia. They may also be obtained from optical measurements.

The other method for determining the distance to a pulsar is by dispersion measure. The dispersion measure, $D M$, is the integrated column density of free electrons between observer and the pulsar. In order to obtain the distance $d$ to a pulsar from the dispersion measure, the mean electron density, $n_{e}$, between the Earth and the pulsar should be known. These parameters are related according to the formula: 


$$
D M=\int_{0}^{d} n_{e} d l \approx n_{e} d
$$

where $d l$ is integration along the column. The dispersion measure is obtained from pulsar timing as the time delay, $\Delta \tau$, between two frequencies $\nu_{1}$ and $\nu_{2}$, with $\nu_{1}<\nu_{2}$, as related by the equation 37 :

$$
\Delta \tau=\frac{e^{2}}{8 \pi^{2} \epsilon_{0} m_{e} c}\left(\nu_{1}^{-2}-\nu_{2}^{-2}\right) .
$$

The number of detected pulsars is limited due to selection biases. Due to the inverse square law, only those objects bright or close enough can be detected. Also, the sky has a radio background from synchrotron radiation electrons in the Galactic magnetic field. The finite angular size of the emission beam, i.e., less than $4 \pi \mathrm{sr}$, also limits the number of pulsars detected. Furthermore, the pulsars may exhibit non-periodic behavior from time to time, a phenomenon known as nulling [38].

\subsection{Choosing Neutron Stars for Analysis}

6 neutron stars were chosen from the Australia Telescope National Facility (ATNF) catalog [39], based on several criteria. The distance should be less than 400 parsecs, since distance degrades the limits by $1 / d^{2}$. Sources were chosen to be greater than $15^{\circ}$ in galactic latitude away from the galactic plane, to avoid background contamination from the diffuse emission and point sources. Fields of less than $2 \times 10^{13}$ gauss were chosen to minimize the attenuation of the potential gamma-ray signal due to gammaray pair production effects, as discussed in Chapter 3. Neutron stars with ages of less than $2 \times 10^{8}$ yr were chosen, since the gravitons decay exponentially with the age of the source, as discussed in the previous chapter. The field was determined as according to equation (4.7). The distribution of sources in the Fermi-LAT 1 year sky is shown in Figure 4.3 


\begin{tabular}{|l|l|l|l|l|l|l|l|l|}
\hline $\begin{array}{l}\text { source } \\
\text { name }\end{array}$ & $\begin{array}{l}\text { RA } \\
\left(^{\circ}\right)\end{array}$ & $\begin{array}{l}\text { Dec. } \\
\left(^{\circ}\right)\end{array}$ & $\begin{array}{l}\ell \\
\left(^{\circ}\right)\end{array}$ & $\begin{array}{l}b \\
\left(^{\circ}\right)\end{array}$ & $\begin{array}{l}\text { Period } \\
(\mathrm{s})\end{array}$ & $\begin{array}{l}d \\
(\mathrm{kpc})\end{array}$ & $\begin{array}{l}\text { Age } \\
(\mathrm{yr})\end{array}$ & $\begin{array}{l}B_{\text {surf }} \\
(\mathrm{G})\end{array}$ \\
\hline RXJ1856-3754 & 284.148 & -37.902 & 358.61 & -17.21 & 7.05 & 0.16 & $3.76 \mathrm{E} 6$ & $1.47 \mathrm{E} 13$ \\
\hline J0108-1431 & 17.035 & -14.351 & 140.93 & -76.82 & 0.808 & 0.24 & $1.66 \mathrm{E} 8$ & $2.52 \mathrm{E} 11$ \\
\hline J0953+0755 & 148.289 & 7.927 & 228.91 & 43.7 & 0.25 & 0.26 & $1.75 \mathrm{E} 7$ & $2.44 \mathrm{E} 11$ \\
\hline J0630-2834 & 97.706 & -28.579 & 236.95 & -16.76 & 1.24 & 0.33 & $2.77 \mathrm{E} 6$ & $3.01 \mathrm{E} 12$ \\
\hline J1136+1551 & 174.014 & 15.851 & 241.90 & 69.20 & 1.19 & 0.36 & $5.04 \mathrm{E} 6$ & $2.13 \mathrm{E} 12$ \\
\hline J0826+2637 & 126.714 & 26.623 & 196.96 & 31.74 & 0.53 & 0.36 & $4.92 \mathrm{E} 6$ & $9.64 \mathrm{E} 11$ \\
\hline
\end{tabular}

Table 4.1: Table of Astrophysical Properties of Neutron Star Sources Analyzed 39].

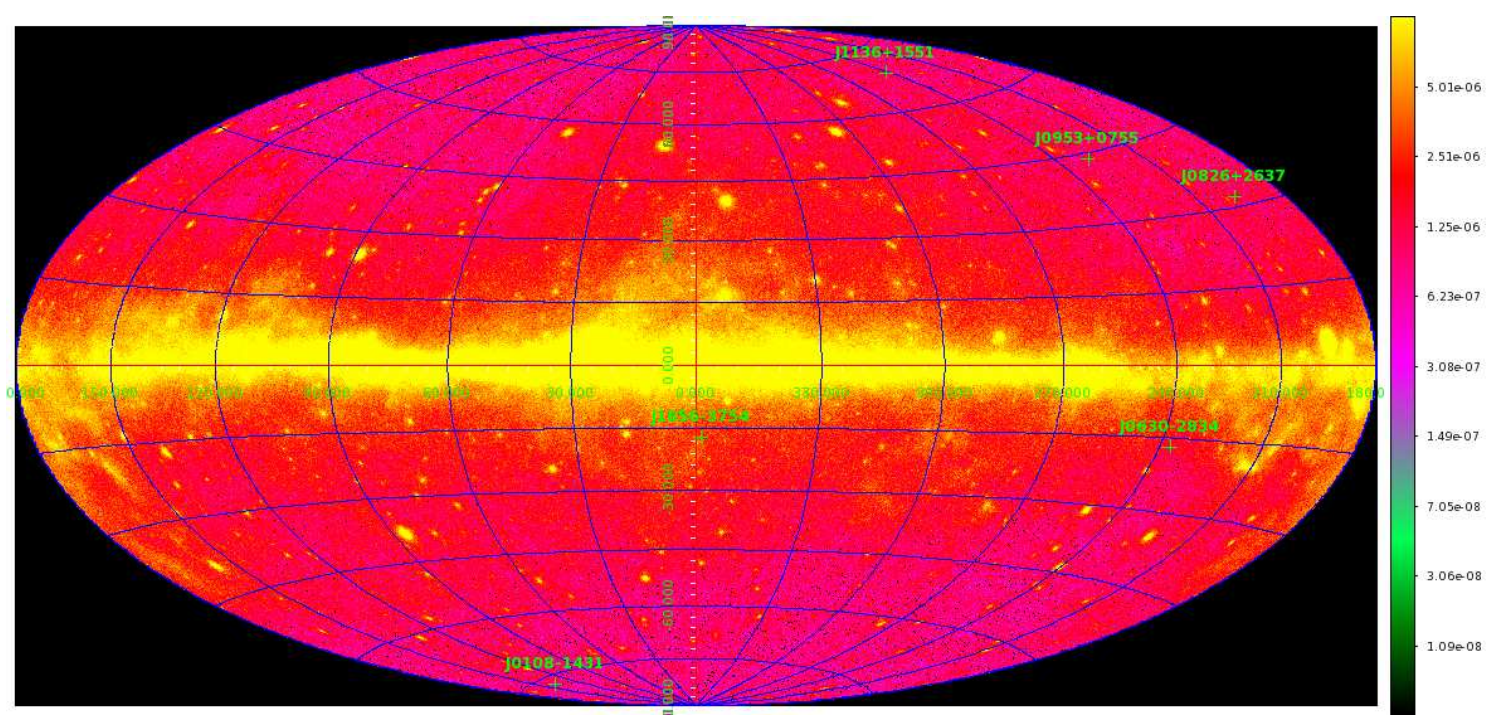

Figure 4.3: 1 yr Fermi sky intensity map $\left(\mathrm{cm}^{-2} \mathrm{~s}^{-1}\right)$, plotted in galactic coordinates, as viewed with ds9 40]. The scale is logarithmic, and the neutron stars selected for LED analysis depicted as green crosses. 


\section{$4.4 \quad$ RXJ1856.35-3754}

This isolated neutron star is the nearest pulsar in the ATNF catalog, at a distance of approximately $0.16 \mathrm{kpc}$. Out of the pulsars studied here, it has the longest period (7.05s) and largest period-dot, which accounts for the larger surface magnetic field. It is relatively young, with an age of $3.76 \mathrm{Myr}$. It was discovered by FM Walter, et al., with ROSAT X-ray data, as an X-ray pulsatol1 41]. It is a radio-quiet source. In addition to observation in X-ray wavelengths, it has also been observed optically with Hubble Space Telescope (HST) observations, which is the basis for various parallax and inferred distance measurements. An initial distance measurement of $60 \mathrm{pc}$ was made by Walter in 2001 based on parallax measurement from the Planetary Camera (PC) aboard HST [42], and Hannestad and Raffelt's 2003 article cited Walter's 2001 measurement. In the erratum to their 2003 paper, Hannestad and Raffelt cited the revised distance measurement of 117 pc determined by Walter and Lattimer, in 2002 43]. The most recent measurement of the distance of $161_{-14}^{+18} \mathrm{pc}$, as cited in the ATNF pulsar catalog, was determined by van Kerkwijk, et al., from optical observations from HST's High Resolution Camera (HRC) of the Advanced Cameras for Surveys (ACS), based on the measured parallax $\pi=6.2 \pm 0.6 \mathrm{mas}[44]$. As compared with the $\mathrm{PC}$, the ACS boasts greater sensitivity and a smaller pixel scale, which renders this measurement more reliable.

The age and magnetic field of this neutron star were determined from timing weak X-ray pulsations observed by XMM-Newton and Chandra 45]. ROSAT measurements didn't reveal pulsations. From timing, it was determined that the period $P=7.05 \mathrm{~s}$ and that the period derivative $\dot{P}=2.97 \times 10^{-14} \mathrm{~s} \mathrm{~s}^{-1}$. The surface magnetic field of $1.5 \times 10^{13} \mathrm{G}$ was derived from equation (4.7). This source is believed to be an aligned rotator, i.e., the spin axis is believed to be parallel to the magnetic dipole axis [46]. In addition, the radius is believed to be $R_{N S} \simeq 14 \mathrm{~km}[47]$, which is close to the nominal value of $R_{N S} \simeq 13 \mathrm{~km}$ that we use.

\footnotetext{
${ }^{1}$ The prefix RX denotes discovery by ROSAT X-ray mission
} 


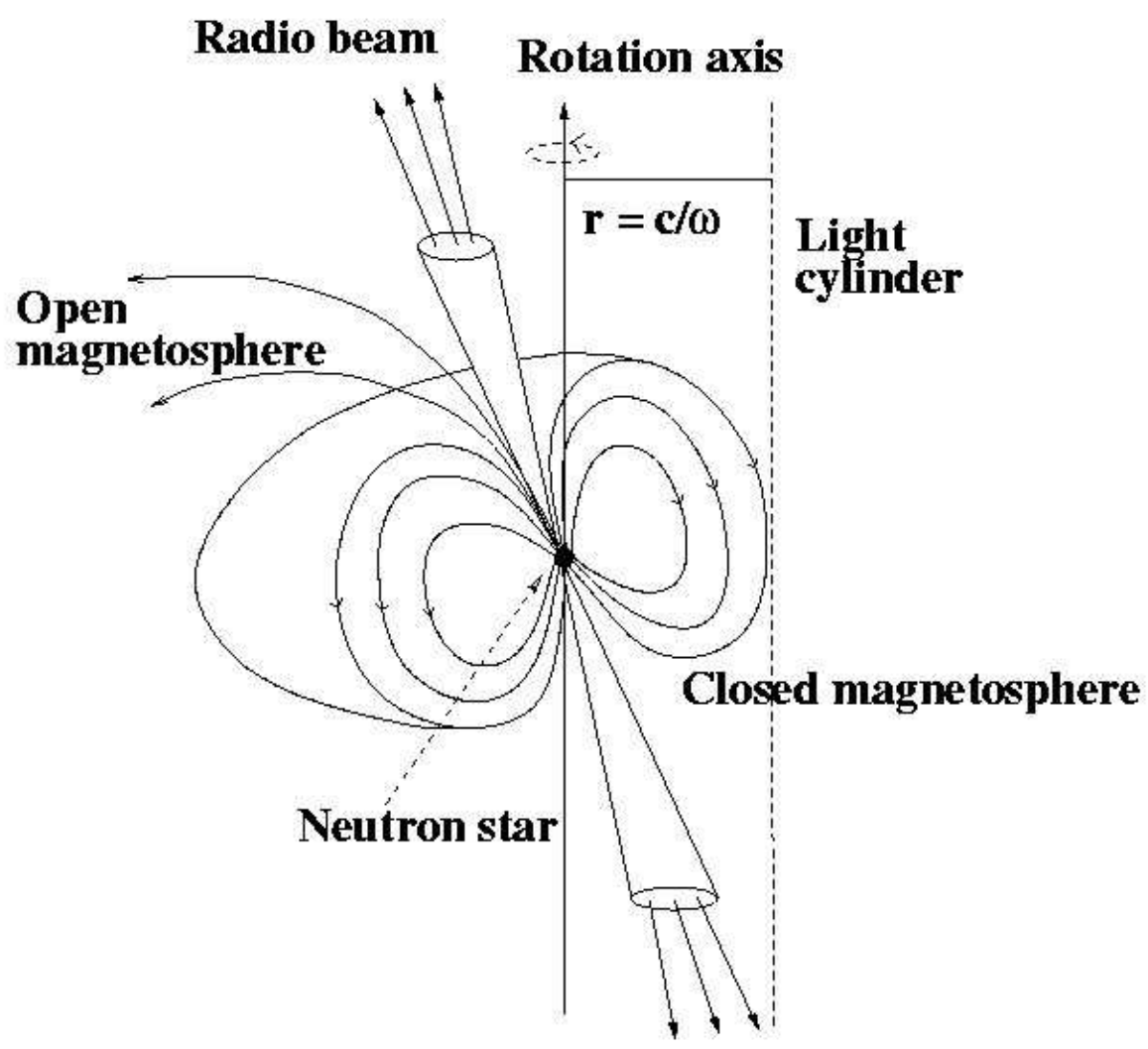

Figure 4.4: A schematic of a pulsar. A magnetosphere surrounds the neutron star, and the magnetic dipole axis makes an angle with the spin axis of rotation, for this oblique rotator [48]. 


\subsection{PSR J0108-1431}

This isolated neutron star was discovered by Tauris, et al., in 1994, in radio observations 49]. It was more recently observed in optical and in X-rays wavelengths 50] [51]. Based on very long baseline interferometry (VLBI) with the Australian Long Baseline Array (LBA), the parallax of this source is $\pi=4.170 \pm 1.421 \mathrm{mas}$, and the corresponding parallax distance of this source is $240_{-61}^{+124} \mathrm{pc} 522^{2}$. LBA observations determining position and parallax for this source were made at a radio frequency of $1650 \mathrm{MHz}$. The dispersion measure (DM) based distance measurements tend to be lower for this source: the latest DM-based distance estimate was $184 \mathrm{pc}$ [53]. The ATNF catalog distances which are cited here are based on parallax data. This source has also been observed in X-ray and optical wavelengths. The observed $\dot{P}$ is $7.704 \times 10^{-17}$, which is the basis for the age estimate of $1.66 \times 10^{8} \mathrm{yr}$. Due to an additional correction to the period-dot due to the proper motion, known as the Shklovsky correction, the age may need to be revised upwards to $2.00 \times 10^{8} \mathrm{yr}[54]$.

\subsection{PSR J0630-2834}

This neutron star, also known as PSR B0628-28, is observed in X-rays, radio, and optical. It was discovered in 1969. The first Molongolo radio survey detected it in 1973. According to VLBI with the LBA, the parallax distance was determined to be $332_{-40}^{+52} \mathrm{pc}[52]$. The dispersion measure distance estimate of $1.45 \mathrm{kpc}$ is much higher than the parallax distance estimate [53]. The ROSAT X-ray pulsating source RXJ0630.8-2834 was believed to be the X-ray counterpart to this source by virtue of proximity, only 1.5 arcseconds apart. Proper motion may account for the slight positional difference [55]. A definite association was not made until later on in 2005, when XMM-Newton observations detected the same pulse period in X-rays as in radio. $P=1.2442$, and $\dot{P}=-4.616 \times 10^{-15}$, from which $B_{\text {surf }}$ of $3 \times 10^{12} \mathrm{G}$ was inferred. It has a very high X-ray luminosity compared with its total luminosity [55]. It is believed to be an oblique rotator, with an inclination between the rotation axis

\footnotetext{
${ }^{2}$ Uncertainties are $1 \sigma$ errors
} 
and magnetic dipole axis of $70^{\circ}[55]$.

\subsection{PSR J0953+0755}

This neutron star, also known as B0950+08, seems to be associated with the remnant of a supernova that occurred in the southern constellation Antlia Pneumatica approximately 1.8 Myr ago, which had a luminosity at Earth of the brightness of the full moon [56]. The distance was believed to be $120 \mathrm{pc}$, based on an estimated parallax of $7.9 \pm 0.8$ mas [57]. Newer VLBI measurements have revised the parallax to $3.82 \pm 0.07$ mas, from which a distance estimate of $262 \pm 5$ pc can be obtained [58]. The age of the supernova remnant Antlia is $1.8 \mathrm{Myr}$, and could very well be the age of PSR J0953+0755, if this neutron star was produced by the original supernova, However, we take the age as the spin-down age for this pulsar, which is estimated as 17.5 Myr, for consistency with the ATNF catalog value.

This source was analyzed by Hannestad and Raffelt in their 2003 paper, in addition to the source PSR J1856-3754[28].

\subsection{PSR J1136+1551}

The pulsar PSR J1136+1551, also known as B1133+16, is a radio pulsar, discovered in 1968. It is also an X-ray-detected rotation-powered field pulsar [46], with a power law X-ray spectrum. A parallax measurement was made with VLBA in 2002, which has remained accurate to the current time [58], revealed the distance to be $350_{-2}^{+2} \mathrm{kpc}$, while $D M$-based distance measures places is at $260 \mathrm{pc}$. This neutron star as been shown to exhibit periodic nulling, as determined by sensitive Arecibo observations [59].

\subsection{PSR J0826+2637}

The pulsar PSR J0826+2637, also referred to as PSR B0823+26, was discovered in 1968. Observed in radio wavelengths, it is also classified as an X-ray pulsator. It has a proper motion of $109 \pm 3$ mas/yr, and a transverse velocity of $184 \mathrm{~km} / \mathrm{s}[39]$. 
The parallax was determined by VLBI measurements as $2.8 \pm 0.6$ mas, from which a distance estimate of $360 \mathrm{pc}$ was obtained [57]. The orbit of this pulsar intersects that of the Per OB3 association; if Per OB3 is in fact the parent of this pulsar, then the age is $\sim 1$ Myr, which is almost a factor of 5 less than the characteristic spin-down age of $4.9 \mathrm{Myr}$ 60]. This difference hardly affects the presumed current number of trapped graviton due to lifetime, according to the simulations in this dissertation. This pulsar is classified as a runaway pulsar, due to its relatively high radial velocity of $100 \mathrm{~km} / \mathrm{s}$. 


\section{Chapter 5}

\section{Data Analysis Methods}

\subsection{Fermi Science Tools}

The Fermi-LAT Science Tools provide the basic software necessary for the analysis of data from the Fermi-LAT. These tools have been well documented on-line, but they will be briefly explained here, with emphasis on those necessary for a unbinned maximum-likelihood analysis. In the analysis done here, the standard publicly released FITS-formatted files have been used for analysis; these are referred to as FT1 and FT2 files. The FITS (Flexible Image Transport System) data format is intended as a means for convenient exchange of astronomical data. A FITS data file consists of a sequence of Header Data Units (HDUs). The first HDU is likely to contain instrument status or the history of the data; subsequent HDUs consist of binary formatted tables/matrices of data or images [61]. Spectral models for point sources and diffuse sources are generally specified by Extensible Markup Language (XML) formatted files.

From the Fermi web server, one may obtain processed data files corresponding to the data acquired by the LAT over a given time range, energy range, coordinate ranges. An FT1 file, also known as an "Event" file, contains the data for each gamma ray event, such as Right Ascension, Declination, energy, zenith angle, time, event class, etc. An FT2 file, also known as a "Spacecraft" file, contains observation start and stop times, and the following variables for 30 second intervals: positions, McIlwain 
parameters, SAA flag, LAT mode, and livetime. After the data in a certain region of the sky has been selected for a certain time interval, the data are processed in a certain way to facilitate analysis. In the analysis done for this dissertation, generally we have used the following scripts: gtselect, gtmktime, gtltcube, gtsrcmaps, and gtexpmap.

\subsubsection{Performing Selections on Data and Making Good Time Intervals}

gtselect can be used to perform sub-selections of a given ft1 event file. select an energy range and a time sub-interval from the FT1 file. Events may be chosen to lie within a radius of a given size of a specified center, within a given time range, and within a given energy range. Next, Good Time Intervals (GTIs) are calculated using the gtmktime utility. A GTI is a time range over which the data may be considered valid, and this extension is made part of the processed event file. Generally, it is desirable for the GTI to filter out events where the spacecraft is located physically in the South Atlantic Anomaly (SAA). The SAA is a region located over South America, the South Atlantic, and Africa where the LAT is exposed to high flux of charged particles, due to the geomagnetic fields of the earth, and is not taking data. In orbit, the LAT crosses over the SAA $15 \%$ of the time; the SAA region is approximated in this mission by a polygonal shaped region defined by vertex points. Events are also excluded for which the rocking angle is greater than $43^{\circ}$ because of albedo contamination from Earth's limb. In addition, a zenith angle cut should also be performed which depends on the size of the ROI and the maximum angle between the local zenith angle and the LAT boresight $\left(105^{\circ}\right)$. This cut also minimizes contamination of the gamma-ray sample arising from interactions of cosmic rays with Earth's limb.

\subsubsection{Generating Livetime Cubes}

Livetime, the inverse of deadtime, is an important consideration for analysis. Every time a gamma ray interacts in the LAT, causing a trigger, further data taking is 
disabled until data from the event are read out. The dead time per event is small, on the order of $20 \mu \mathrm{s}$, but the LAT has a kHz trigger rate on cosmic rays, so the fraction of time LAT will be dead is on the order of $10 \%$. Knowing the livetime, along with the effective area, allows the rate of events from a source to be converted into flux units.

The script gtltcube is used to make a livetime cube, also known as an exposure cube, taking as arguments the FT1 and FT2 files, the desired bin size in degrees per pixel, and the cosine of inclination angle. The exposure cube is a precomputed file which speeds up the likelihood based fitting computations. It is calculated on a spatial HEALPix 1 grid; this pixelization scheme produces a subdivision of a spherical surface in which each pixel covers the same surface area as every other one [62]. Furthermore, the center of each pixel lies on a discrete number of constant-latitude rings, with the number of constant-latitude rings being dependent on the HEALPix grid resolution. Livetime cubes are additive over different time ranges: e.g., the livetime cube for 1 year could be the sum of monthly livetime cubes, as computed with the ScienceTools script gtltsum.

\subsubsection{Generating Counts Maps}

The script gtbin bins the data into counts maps; a counts map is an image depicting the number of counts in spatial bins. In the simple 2D counts map (CMAP) mode, all events are binned by spatial bins according to the number of pixels and the location of the image center. Common astronomical coordinate systems used include equatorial (RA,Dec) and galactic $(\ell, b)$. The projection may also be specified: common projections include Hammer-Aitoff or Cartesian. In the 3D counts map mode, as specified by CCUBE, the data is binned as for the $2 \mathrm{D}$ counts map, but differs in that a different 2D counts map is generated for energy ranges as specified by an energy binning scheme which may be linear, logarithmic, or user-defined. When visualized by a viewer such Smithsonian Astrophysical Observatory's (SAO) ds9 program [40], a color scale may often be selected for a false color image, with each color representing

\footnotetext{
${ }^{1}$ Hierarchical Equal Area isoLatitude Pixelization
} 
a different number of counts per bin. Linear scale is the simplest, but square-root and log-scale are often more useful.

The script gtsrcmaps convolves source model components with the instrument response function, a prerequisite for creating a model map. Given a specified source model in Extensible Markup Language (XML) format, a spacecraft file, an exposure cube, a counts map, and an IRF, one can obtain an output of a binned exposure map and a source map in FITS format. Point sources as well as diffuse components should be specified in the XML file.

\subsubsection{Generating Exposure Maps}

The FITS file produced by the script gtexpmap is a prerequisite for unbinned analysis. The exposure map gives the total exposure - LAT effective area area multiplied by corrected time $\left(\mathrm{cm}^{2} \mathrm{~s}\right)$ - over positions in the sky, producing counts. The gtexpmap script takes as arguments the size of the source region (generally larger than the ROI size), the number of pixels, the number of energies, and the FT1 file(s), FT2 file(s), the livetime cube, and the set of IRFs used. Formally, gtexpmap is calculating the exposure at each energy $\epsilon$ and position $\hat{p}$ according to the integral of the total response $R$ over the ROI data-space:

$$
\epsilon(E, \hat{p})=\int_{R O I} d E^{\prime} d \hat{p}^{\prime} d t R\left(E^{\prime}, \hat{p^{\prime}} ; E, \hat{p}, t\right)
$$

The exposure function, $\epsilon(E, \hat{p})$, may then be used along with the photon intensity of the source $i, S_{i}(E, \hat{p})$, to calculate the predicted number of events from a given source, $N_{\text {pred }}$, as:

$$
N_{\text {pred }}=\int d E d \hat{p} S_{i}(E, \hat{p}) \epsilon(E, \hat{p})
$$

In contrast to the livetime cube, which is calculated on a HEALPix grid, the exposure map is calculated on a galactic longitude-latitude grid. 


\section{$5.2 \quad$ Model Fitting}

\subsubsection{The Log-Likelihood Function}

The binned likelihood function can be expressed as:

$$
\mathcal{L}=\prod_{i} \frac{M_{i}^{n_{i}} e^{-M_{i}}}{n_{i} !}
$$

where $M_{i}$ are the predicted counts in each bin, and $n_{i}$ are the detected counts in each bin. This is essentially of product of probabilities, based upon the assumption that the observed number of counts in each bin may be characterized by a Poisson distribution. This holds in the case of a small number of counts. In the case of unbinned likelihood, the bin sizes get infinitesimally small, such that $n_{i}$ is either 0 or 1. Thus, the unbinned likelihood function can be expressed as:

$$
\mathcal{L}=\prod_{i} M_{i} e^{-M_{i}},
$$

and the corresponding log-likelihood function is given by

$$
\log \mathcal{L}=\sum_{i} \log \left(M_{i}\right)-N_{\text {pred }},
$$

where the model counts corresponding to source $i$ are given by in general as:

$$
M_{i}=\int d E d p R\left(E^{\prime}, \hat{p}^{\prime} ; E, \hat{p}, t\right) S_{i}\left(E, \hat{p} ; \alpha_{i}\right) .
$$

$S_{i}\left(E, \hat{p} ; \alpha_{i}\right)$ represents the source intensity of source $i$ at energy $E$ and position $\hat{p}$, $R\left(E^{\prime}, \hat{p}^{\prime} ; E, \hat{p}, t\right)$ is the instrument response function, and $\alpha_{i}$ is a parameter describing the source intensity. The predicted counts, $N_{\text {pred }}$, over the ROI is related to the model counts and is given by:

$$
N_{\text {pred }}=\int_{R O I} d E^{\prime} d p^{\prime} M_{i}\left(E^{\prime}, p^{\prime}\right)
$$




\subsubsection{Test Statistic}

The test statistic (TS), a measure of the significance of a given source detected at a given position, is proportional to the ratio of likelihoods. It is formally defined as:

$$
T S=-2 \ln \left(L_{\max , 0} / L_{\max , 1}\right)
$$

where $L_{\max , 0}$ is the maximum likelihood without a given source (the null hypothesis) and $L_{\max , 1}$ is the maximum likelihood with the given source. For the Gaussian approximation, the TS is expected to vary roughly as the square of the statistical significance in number of $\sigma$. Likelihood-based analysis of data in photon-counting experiments was explained by Cash [63]; in gamma-ray astrophysics, likelihood based analysis has precedent in parameter estimation in analysis of EGRET data 64].

\subsubsection{Fitting with gtlike}

The script gtlike performs unbinned and binned likelihood analysis. Unbinned analysis may be used when it is preferable that each event contribute to the likelihood function independent of bin: this is generally a more memory-intensive task when the number of photon events becomes large. A source model file, binned exposure map, source map, a 3D-counts map, and a livetime cube should be provided when running in binned likelihood mode. In unbinned likelihood mode, event files, spacecraft files, an exposure map (as produced by gtexpmap), a livetime cube, and IRFs need to be specified. The fitting algorithm should also be specified as an argument: available routines include MINUIT, NEWMINUIT, DRMNGB. The MINUIT algorithm was used by the catalog contributors and in this analysis. It is a general high-energy physics fitting interface originally written in FORTRAN. Maximizing the likelihood is based on evaluation of the function in the multidimensional free parameter space using numerical derivatives. As output to the likelihood fitting, counts over the entire ROI for each component, and the sum over all components as compared with model, may be shown; in addition, a counts residual plot may be created for comparing data counts versus model counts over the entire ROI. An output XML file corresponding to the fitted model, with the same components as the input XML file, may also be 
generated. Integral fluxes and spectral indices for each point source may be visible in this model, for power law sources.

\subsubsection{Likelihood based fitting with python interface}

The pyLikelihood script is similar to gtlike in its fitting algorithm, but has a object-oriented python interface and has additional features to enhance flexibility in fitting. For example, parameters may be free initially for an initial fit, and then fixed for a second fit by an available option.

\subsubsection{Using Fitted Model}

After generating a fitted model, two ScienceTools scripts are useful. The script gtmodel takes a fitted model specification, and binned exposure map, and generates a counts map for the same region as the data counts map: this is useful for assessing the agreement of data with the fit. One may also be curious to assess the significance of the sources visually: the script gttsmap is useful for generating a map of the test statistic at each bin.

\subsubsection{Implementing a Custom Model for Fitting}

For this dissertation, a custom model was implemented in a special build of the Fermi Science Tools. I noticed that the Monte Carlo model was fitted fairly well with a power law super-exponential cutoff model. Fits are shown in the Appendix.

Two additional parameters were added to the power law super-exponential cutoff model, Const1 and Dist parameters. The size of the extra dimensions is related to terms of Const 1 and $k_{n}$ by this relation:

$$
R^{n}=\text { Const } 1 / k_{n},
$$

where $k_{n}$ is defined in Chapter 3. The differential flux distribution function was 
parametrized as:

$$
\frac{d \Phi}{d E}=\operatorname{Const1}(\text { Dist })^{-2} N_{0}\left(\frac{E}{E_{0}}\right)^{\gamma_{1}} \exp \left(-\left(\frac{E}{E_{c}}\right)^{\gamma_{2}}\right)
$$

Const1 was defined as an intermediate parameter in order to avoid convergence issues: $R^{7}$ may achieve values of $10^{-98}$, which is problematic for the MINUIT optimizer, considering that the floating point values may achieve a minimum of $10^{-100}$. This was the only parameter allowed free in the fitting of the faint neutron star ROI when computing upper limits via MINOS errors. According to this spectral model, the model counts are given by:

$$
M_{\mathrm{NS}_{j}}\left(E^{\prime}, \hat{p}^{\prime}, \operatorname{Dist}_{j}, \operatorname{Const}_{j}, n\right)=\int_{i} d E d \hat{p} d t R\left(E^{\prime}, \hat{p}^{\prime} ; E, \hat{p}, t\right) \frac{d \Phi\left(\operatorname{Dist}_{j}, \operatorname{Const}_{j}, n\right)}{d E}
$$

and the total predicted model counts over the ROI are given by the integral of the model counts from each source.

In order to use the MINUIT optimizer, it is necessary for the derivative of the differential flux distribution function with respect to fitted parameters to be specified. Since the differential flux function varies linearly with Const1, the derivative of the function with respect to Const1 is given by:

$$
\frac{d}{d \text { Const1 }} \frac{d \Phi}{d E}=\frac{1}{\text { Const1 }} \frac{d \Phi}{d E}
$$

\subsection{Computing Upper Limits with MINOS Errors}

MINOS errors were used to calculate upper limits at the desired confidence level 65$]$. The resulting MINOS errors are used to calculate errors of the single free parameter from the fitted model as determined by MINUIT. They take into account nonlinearities and, in the case of multiple free parameters, parameter correlations. The MINOS level is defined as that value of the function $-2 \Delta \log \mathcal{L}(p)$, such that when reached for a parameter value $\mathrm{p}$, MINUIT return as the MINOS error the value 
$p-p_{\min }$, where $p_{\min }$ is the fitted value of the parameter. In other words, suppose that the $\log$-likelihood function minimum has the value $\mathcal{L}_{\text {min }}$; as the parameter is varied by $p-p_{\text {min }}$, the minimum of the log-likelihood takes on the fitted value $\mathcal{L}_{\text {min }}+$ level $/ 2$. The specification of the level argument indicates the confidence level desired. Based on the expectation of Gaussian errors, the level argument is supposed to vary quadratically with the MINOS error. A one standard deviation error corresponds to a level of 1 , and a two standard deviation error corresponds to a level of 4, i.e., an $n$-standard deviation error corresponds to a level of $n^{2}$. The one-sided 95\% confidence level (CL) upper limit, which we use in this work, corresponds to level $=2.71[\underline{8}]$.

\subsection{Combining Limits}

\subsubsection{Using MINOS Errors}

MINOS errors were also used to combine limits from multiple targets as well. For each $n$, curves of level vs $R^{n}$ for each of the sources were summed, to yield a combined limit curve. This is analogous to summing over the log-likelihoods of the ROIs, since the MINOS curve contains information about the log-likelihood. The intersection of the summed curve with a level of 2.71 gives the combined upper limit for a one-sided 95\% CL, which is better than the limit from any individual source.

\section{CompositeLikelihood}

Combining results from multiple ROIs by stacking is possible within the framework of pyLikelihood. Using a module called CompositeLikelihood, common parameter from multiple ROIs are tied together [66]. In our case, the common parameter of interest is related to $R^{n}$. The sum of the log-likelihoods over the ROIs is maximized. Formally, this may be expressed as:

$$
\log \mathcal{L}=\sum_{k} \log \mathcal{L}_{k},
$$

where $k$ is the integer index over the ROIs, and 


$$
\log \mathcal{L}_{k}=\sum_{j_{k}} \log \left(\sum_{i_{k}} M_{i_{k} j_{k}}\left(\alpha_{k}\right)\right)-N_{\text {pred,k}}
$$

The $i_{k}$ s label the sources, while the $j_{k} \mathrm{~s}$ label the counts in region $\mathrm{k}$. MINOS errors may also be computed with the tied parameter $\alpha_{k}$ across the ROIs. By virtue of stacking, the MINOS-error based upper limit may be more stringent than the individual limits for each ROI. This tool has been extensively tested and validated by the Fermi-LAT collaboration.

Overall, the results from combining limits are more stringent than any individual source. The results from the two methods of combining limits are comparable.

\subsection{Computing Limits with Profile Likelihood}

As an additional upper limit finding tool, the UpperLimits module accessible to $p y$ Likelihood computes limits based on the profile likelihood method. In this approach, the profile likelihood algorithm finds the value of the likelihood function at each value of the parameter of interest $\alpha$, maximized over all the other "nuisance" parameters. Nuisance parameters are parameters whose true value are unknown, due to statistical or systematic uncertainties.

In the UpperLimits implementation, the profile likelihood is assumed to obey a chi-squared distribution, and the upper limit is obtained when the profile likelihood decreases by a certain amount from its maximum likelihood estimate. The one-sided confidence region at the $95 \%$ confidence level is given by a decrease of $2.71 / 2$. 


\section{Chapter 6}

\section{Modeling ROIs and Setting Limits on LED}

\subsection{Data Sample in Analysis}

Gamma ray data, i.e., photon events, were extracted from a $12^{\circ}$ circular region around the coordinates of each neutron star from the 1 year catalog dataset. The time interval corresponds to August 4, 2008 to July 4, 2009. This dataset excluded events for which the rocking angle was larger than $43^{\circ}$, due to albedo contamination from Earth's limb. In addition, events for which the zenith angle was larger than $105^{\circ}$ were excluded from the dataset, in order to minimize gamma-ray contamination arising from interactions of cosmic rays with Earth's atmosphere. The cumulative livetime of the all-sky dataset was 245.6 days.

\subsection{Modeling ROIs}

Background point sources from the $1 F G L$ catalog within $14^{\circ}$ radius of each neutron star of interest were modeled as power law function, of the form:

$$
\frac{d N}{d E}=\mathcal{I} \frac{E^{-\Gamma}}{E_{\max }^{-\Gamma+1}-E_{\min }^{-\Gamma+1}} \mathrm{~cm}^{-2} \mathrm{~s}^{-1} \mathrm{MeV}^{-1}
$$


where $\mathcal{I}$ is the integral flux $\left(\mathrm{cm}^{-2} \mathrm{~s}^{-1}\right)$ between $E_{\min }$ and $E_{\max }$, and $\Gamma$ is the spectral index of the source.

The extragalactic isotropic and galactic diffuse components were modeled as well. The extragalactic isotropic component was modeled from the file isotropic_iem_v02.txt, with a normalization determined by fitting in each ROI. The galactic diffuse component was modeled as the collaboration-developed MapCubeFunction gll_iem_v02.fit. It was corrected by a power law, with a prefactor, and a spectral index allowed to vary between -0.1 and 0.1 , analogous to the procedure in determining the 1 year catalog, The galactic diffuse model was corrected in each ROI by a power law as described in the first year catalog paper, making the spectrum effectively harder or softer. The diffuse parameters are summarized in Table 6.1.

The diffuse models were carefully developed by members of the collaboration. The galactic diffuse emission model, gll_iem_v02.fit, was based on spectral line surveys of HI and CO (as a HII tracer) as well as infrared tracers of dust column density. The $\gamma$-ray emissivities of galactocentric rings in several energy bands were fit to LAT observations, given an model of inverse Compton emission that was calculated using GALPROP [67] 68]. The isotropic model, isotropic_iem_v02.txt, was determined as the residual of a fit of the Galactic diffuse emission model to the LAT data at Galactic latitudes for $|b|>30^{\circ}$; it includes contribution from residual cosmic rays at high energies.

The table of parameters for catalog sources near the sources RXJ1856-3754, PSR J0108-1431, PSR J0953+0755,PSR J0630-2834, PSR J1136+1551, and PSR J0826+2637 are listed in Tables B.1, B.2 B.3 B.4, B.5, and B.6. The counts maps compared with the model maps for the 1FGL catalog for the sources RXJ1856-3754, PSR J0108-1431, PSR J0953+0755, PSR J0630-2834, PSR J1136+1551, PSR J0826+2637 are displayed in Figures 6.2, 6.4, 6.5, 6.6 6.7, and 6.8. In all cases, the counts map has been convolved with a Gaussian approximation to the LAT PSF, at 3 different linear bins from 100 to $400 \mathrm{MeV}$, with front and back converting events treated separately 1

\footnotetext{
${ }^{1}$ This was performed using the fgauss utility from NASA's FITS file utilities package FTOOLS.
} 


\begin{tabular}{|l|l|l|l|}
\hline $\begin{array}{l}\text { source } \\
\text { name }\end{array}$ & $\begin{array}{l}\text { Galactic } \\
\text { Prefactor }\end{array}$ & $\begin{array}{l}\text { Galactic } \\
\text { Index }\end{array}$ & $\begin{array}{l}\text { Extragalactic Isotropic } \\
\text { Normalization }\end{array}$ \\
\hline RXJ1856-3754 & 0.9008 & 0.0594 & 0.6651 \\
\hline J0108-1431 & 1.0309 & 0.1000 & 0.6266 \\
\hline J0953+0755 & 1.3417 & 0.1000 & 0.6689 \\
\hline J0630-2834 & 0.9138 & 0.1000 & 0.7416 \\
\hline J1136+1551 & 1.2036 & 0.1000 & 0.7937 \\
\hline J0826+2637 & 1.516 & -0.0085 & 0.5959 \\
\hline
\end{tabular}

Table 6.1: Table of diffuse parameters for ROIs corresponding to each source.
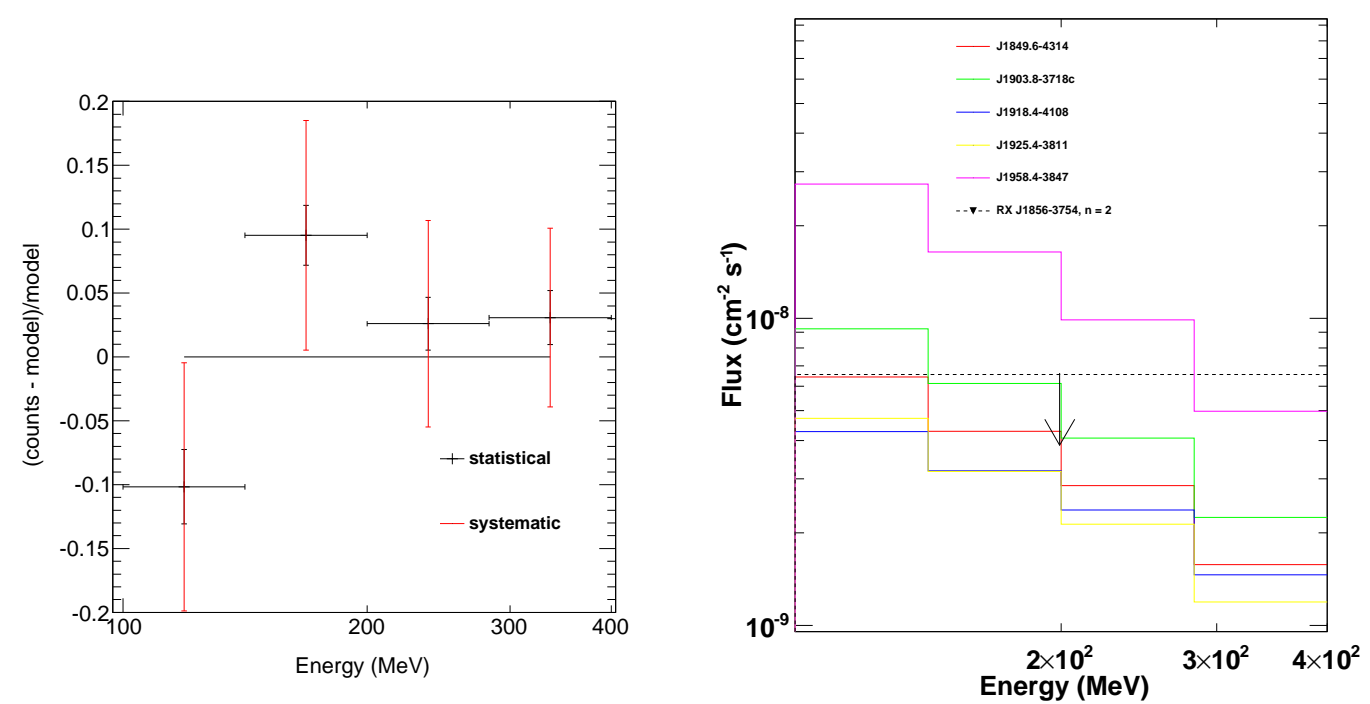

(a) Residual of counts. Black error bars are sta- (b) Fluxes in 4 log-spaced energy bands for tistical, and red error bars are systematic un- background sources.

certainties in this energy range, approximately $10 \%$.

Figure 6.1: Counts residuals plot and fluxes for 1FGL sources within $7^{\circ}$ of RXJ18567354. 

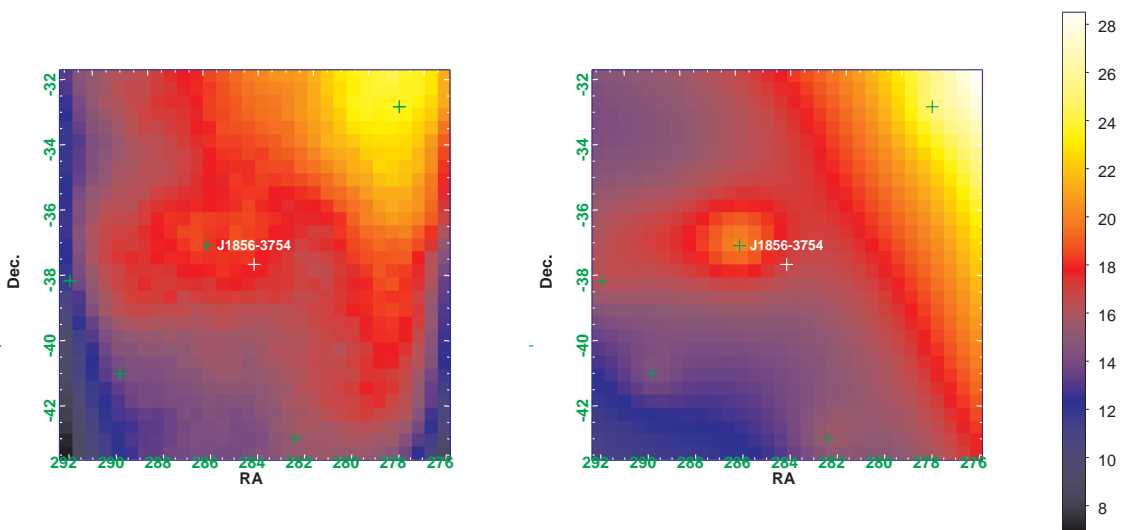

Figure 6.2: Counts map (left) compared with model map (right) for source RXJ1856.35-3754.

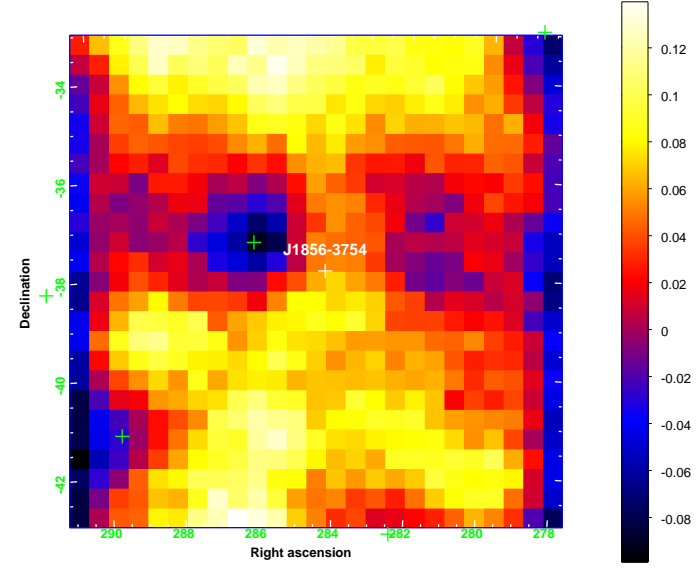

Figure 6.3: Counts residual map for source RX J1856.35-3754. This map was produced by applying the operation (Counts-Model)/Model per pixel.

\begin{tabular}{|c|c|c|c|c|c|c|}
\hline $\mathrm{n}$ & $\mathrm{J} 1856-3754$ & J0108-1431 & J0953+0755 & J1136+1551 & J0630-2834 & J0826+2637 \\
\hline 2 & $3.8 \mathrm{E}-9$ & $4.3 \mathrm{E}-9$ & $5.3 \mathrm{E}-9$ & $4.1 \mathrm{E}-9$ & $5.8 \mathrm{E}-9$ & $6.6 \mathrm{E}-9$ \\
\hline 3 & $4.0 \mathrm{E}-9$ & $4.4 \mathrm{E}-9$ & $5.4 \mathrm{E}-9$ & $4.2 \mathrm{E}-9$ & $6.8 \mathrm{E}-9$ & $8.4 \mathrm{E}-9$ \\
\hline 4 & $3.7 \mathrm{E}-9$ & $4.2 \mathrm{E}-9$ & $6.2 \mathrm{E}-9$ & $4.4 \mathrm{E}-9$ & $9.4 \mathrm{E}-9$ & $9.9 \mathrm{E}-9$ \\
\hline 5 & $4.0 \mathrm{E}-9$ & $4.1 \mathrm{E}-9$ & $6.3 \mathrm{E}-9$ & $4.4 \mathrm{E}-9$ & $1.1 \mathrm{E}-8$ & $1.3 \mathrm{E}-8$ \\
\hline 6 & $4.1 \mathrm{E}-9$ & $4.0 \mathrm{E}-9$ & $6.7 \mathrm{E}-9$ & $4.2 \mathrm{E}-9$ & $1.4 \mathrm{E}-8$ & $1.5 \mathrm{E}-8$ \\
\hline 7 & $4.2 \mathrm{E}-9$ & $4.0 \mathrm{E}-9$ & $7.8 \mathrm{E}-8$ & $3.5 \mathrm{E}-9$ & $1.9 \mathrm{E}-8$ & $1.7 \mathrm{E}-8$ \\
\hline
\end{tabular}

Table 6.2: Table of 95\% CL flux upper limits $\left(\mathrm{cm}^{-2} \mathrm{~s}^{-1}\right)$ for the sources analyzed. 

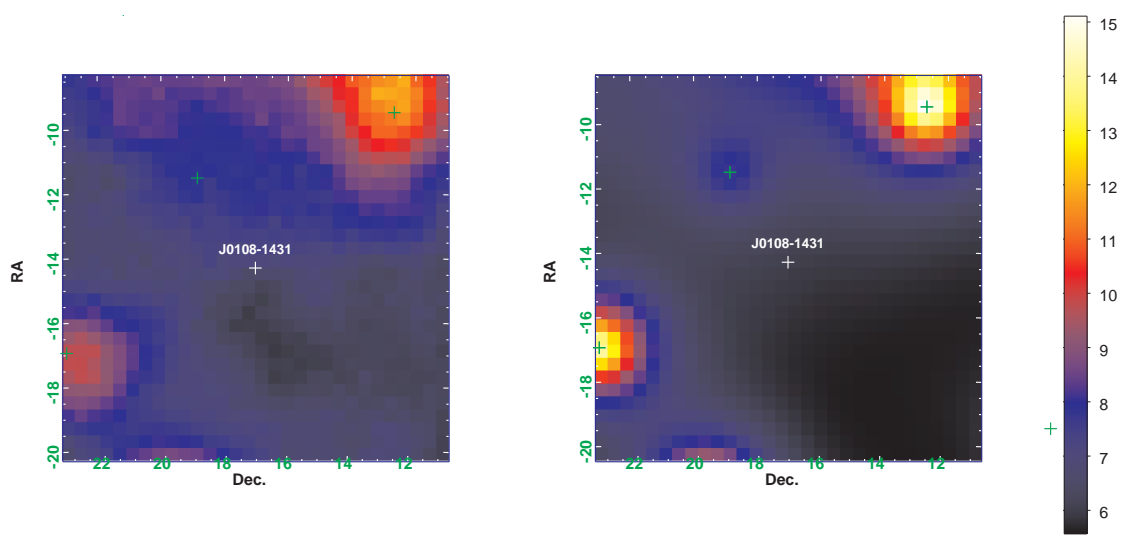

Figure 6.4: Counts map (left) compared with model map (right) for source PSR J0108-1431
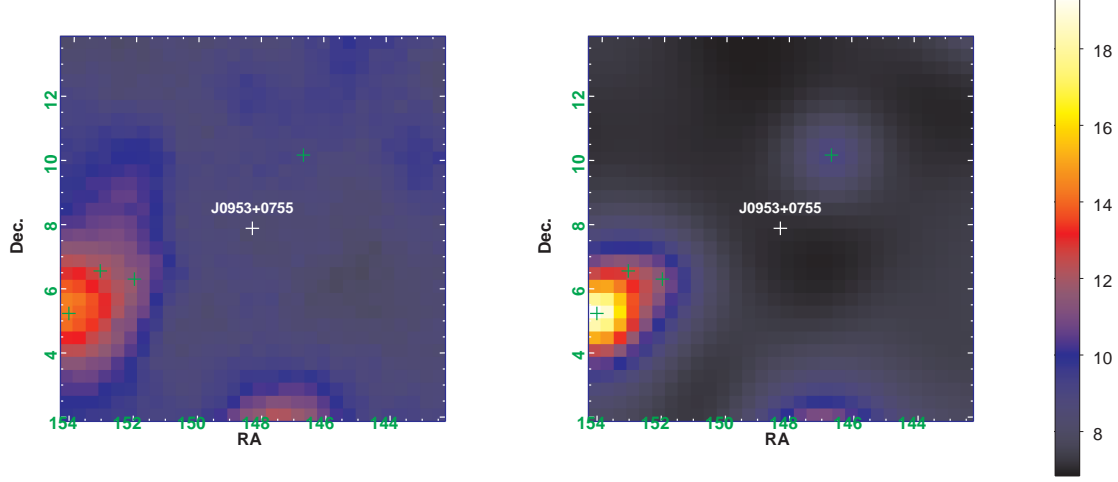

Figure 6.5: Counts map compared with model map for source PSR J0953+0755.

\begin{tabular}{|l|l|l|l|l|l|l|}
\hline $\mathrm{n}$ & J1856-3754 & J0108-14 & J0953+0755 & J1136+1551 & J0630-2834 & J0826+2637 \\
\hline 2 & $9.5 \mathrm{E}-09$ & $4.9 \mathrm{E}-08$ & $2.2 \mathrm{E}-08$ & $2.3 \mathrm{E}-08$ & $2.4 \mathrm{E}-08$ & $2.9 \mathrm{E}-08$ \\
\hline 3 & $3.9 \mathrm{E}-11$ & $1.1 \mathrm{E}-10$ & $6.7 \mathrm{E}-11$ & $6.9 \mathrm{E}-11$ & $7.4 \mathrm{E}-11$ & $8.4 \mathrm{E}-11$ \\
\hline 4 & $2.5 \mathrm{E}-12$ & $5.4 \mathrm{E}-12$ & $3.8 \mathrm{E}-12$ & $3.9 \mathrm{E}-12$ & $4.3 \mathrm{E}-12$ & $4.8 \mathrm{E}-12$ \\
\hline 5 & $5.0 \mathrm{E}-13$ & $9.1 \mathrm{E}-13$ & $7.0 \mathrm{E}-13$ & $7.1 \mathrm{E}-13$ & $8.1 \mathrm{E}-13$ & $8.6 \mathrm{E}-13$ \\
\hline 6 & $1.7 \mathrm{E}-13$ & $2.8 \mathrm{E}-13$ & $2.3 \mathrm{E}-13$ & $2.3 \mathrm{E}-13$ & $2.7 \mathrm{E}-13$ & $2.8 \mathrm{E}-13$ \\
\hline 7 & $8.2 \mathrm{E}-14$ & $1.3 \mathrm{E}-13$ & $1.0 \mathrm{E}-13$ & $1.0 \mathrm{E}-13$ & $1.2 \mathrm{E}-13$ & $1.3 \mathrm{E}-13$ \\
\hline
\end{tabular}

Table 6.3: Table of upper limits on extra dimensions size $R(\mathrm{~m})$ for the sources analyzed. 

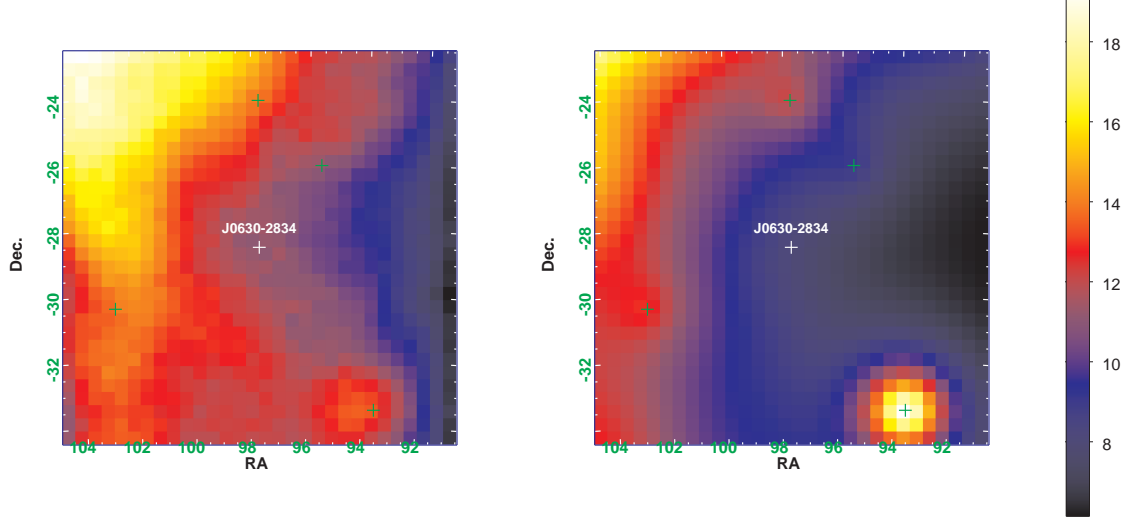

Figure 6.6: Counts map (left) compared with model map (right) for source PSR J0630-2834.
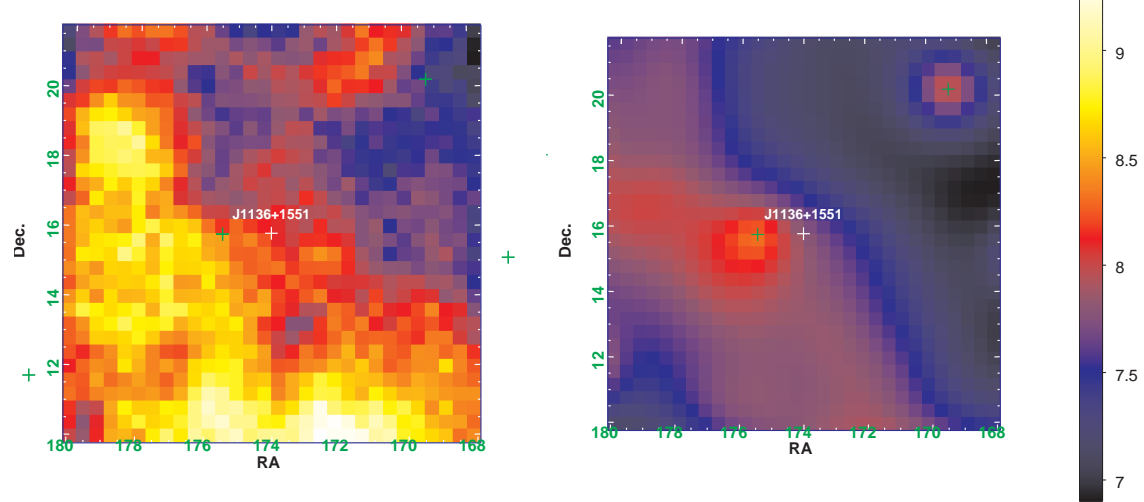

Figure 6.7: Counts map (left) compared with model map (right) for source PSR $\mathrm{J} 1136+1551$.

\begin{tabular}{|l|l|l|l|l|l|l|}
\hline $\mathrm{n}$ & $\mathrm{J} 1856-3754$ & $\mathrm{~J} 0108-14$ & $\mathrm{~J} 0953+0755$ & $\mathrm{~J} 1136+1551$ & $\mathrm{~J} 0630-2834$ & $\mathrm{~J} 0826+2637$ \\
\hline 2 & 210 & 91 & 140 & 130 & 130 & 120 \\
\hline 3 & 14 & 8 & 10 & 10 & 10 & 9.0 \\
\hline 4 & 2.3 & 1.4 & 1.8 & 1.7 & 1.6 & 1.5 \\
\hline 5 & 0.63 & 0.41 & 0.50 & 0.49 & 0.46 & 0.44 \\
\hline 6 & 0.23 & 0.16 & 0.19 & 0.19 & 0.17 & 0.17 \\
\hline 7 & 0.11 & 0.078 & 0.091 & 0.091 & 0.081 & 0.080 \\
\hline
\end{tabular}

Table 6.4: Table of 95\% CL lower limits on $M_{D}(\mathrm{TeV})$ for the individual NS sources. 

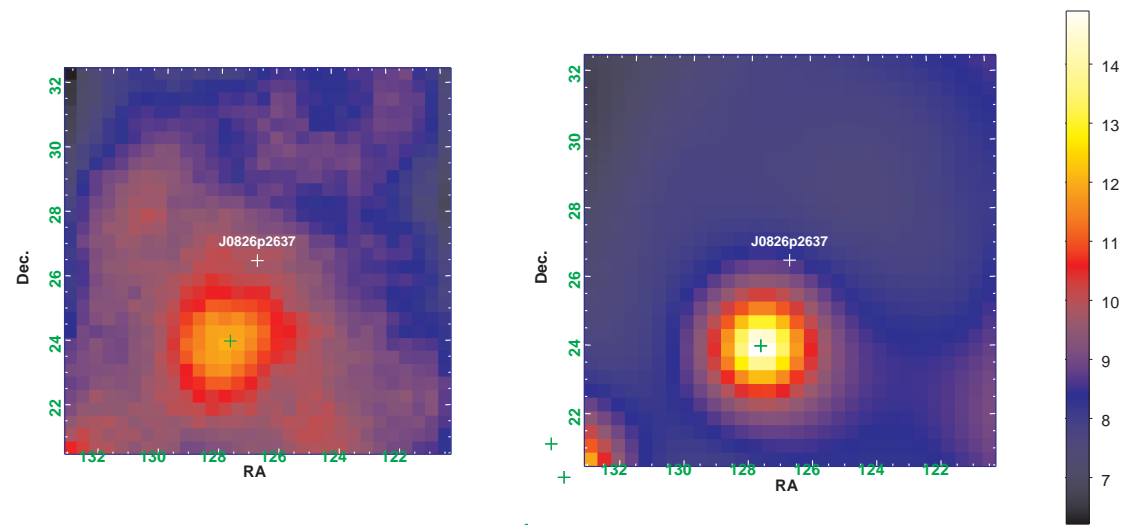

Figure 6.8: Counts map (left) compared with model map (right) for source PSR $\mathrm{J} 0826+2637$.

\begin{tabular}{|l|l|l|l|l|}
\hline $\mathrm{n}$ & $\begin{array}{l}\text { HR2003 } \\
(\text { SN1987A) }\end{array}$ & $\begin{array}{l}\text { HR2003 } \\
\text { (NS) }\end{array}$ & $\begin{array}{l}\text { Combined } \\
\text { (MINOS) }\end{array}$ & $\begin{array}{l}\text { Combined } \\
\text { (Composite) }\end{array}$ \\
\hline 2 & $9.6 \mathrm{E}-7$ & $5.1 \mathrm{E}-8 \mathrm{E}$ & $7.5 \mathrm{E}-9$ & $7.6 \mathrm{E}-9$ \\
\hline 3 & $1.1 \mathrm{E}-9$ & $1.1 \mathrm{E}-10$ & $3.3 \mathrm{E}-11$ & $3.3 \mathrm{E}-11$ \\
\hline 4 & $3.8 \mathrm{E}-11$ & $5.5 \mathrm{E}-12$ & $2.3 \mathrm{E}-12$ & $2.3 \mathrm{E}-12$ \\
\hline 5 & $4.9 \mathrm{E}-12$ & $9.1 \mathrm{E}-13$ & $4.7 \mathrm{E}-13$ & $4.7 \mathrm{E}-13$ \\
\hline 6 & $1.2 \mathrm{E}-12$ & $2.8 \mathrm{E}-13$ & $1.6 \mathrm{E}-13$ & $1.7 \mathrm{E}-13$ \\
\hline 7 & $4.4 \mathrm{E}-13$ & $1.2 \mathrm{E}-13$ & $7.8 \mathrm{E}-14$ & $7.9 \mathrm{E}-14$ \\
\hline
\end{tabular}

Table 6.5: Comparison of 95\% CL upper limits on the size of extra dimensions, $R$. Previous limits obtained in the article by Hannestad and Raffelt in 2003 are compared with combined limits from the sources.

\subsection{Limits Results}

\subsubsection{Individual Limits}

Individual flux upper limits and upper limits on $R(\mathrm{~m})$ are obtained from the loglikelihood function, as described in Chapter 5. Limits were combined with posterior PDFs, derived from the MINOS error curves from each source, as well as CompositeLikelihood. MINOS error curves are shown in Figure 6.9. Results of 95\% CL upper limits from posterior PDFs and stacking as compared with previous results are presented in Table 6.5. 95\% CL lower limits on $M_{D}$ are shown in Table 6.6. 


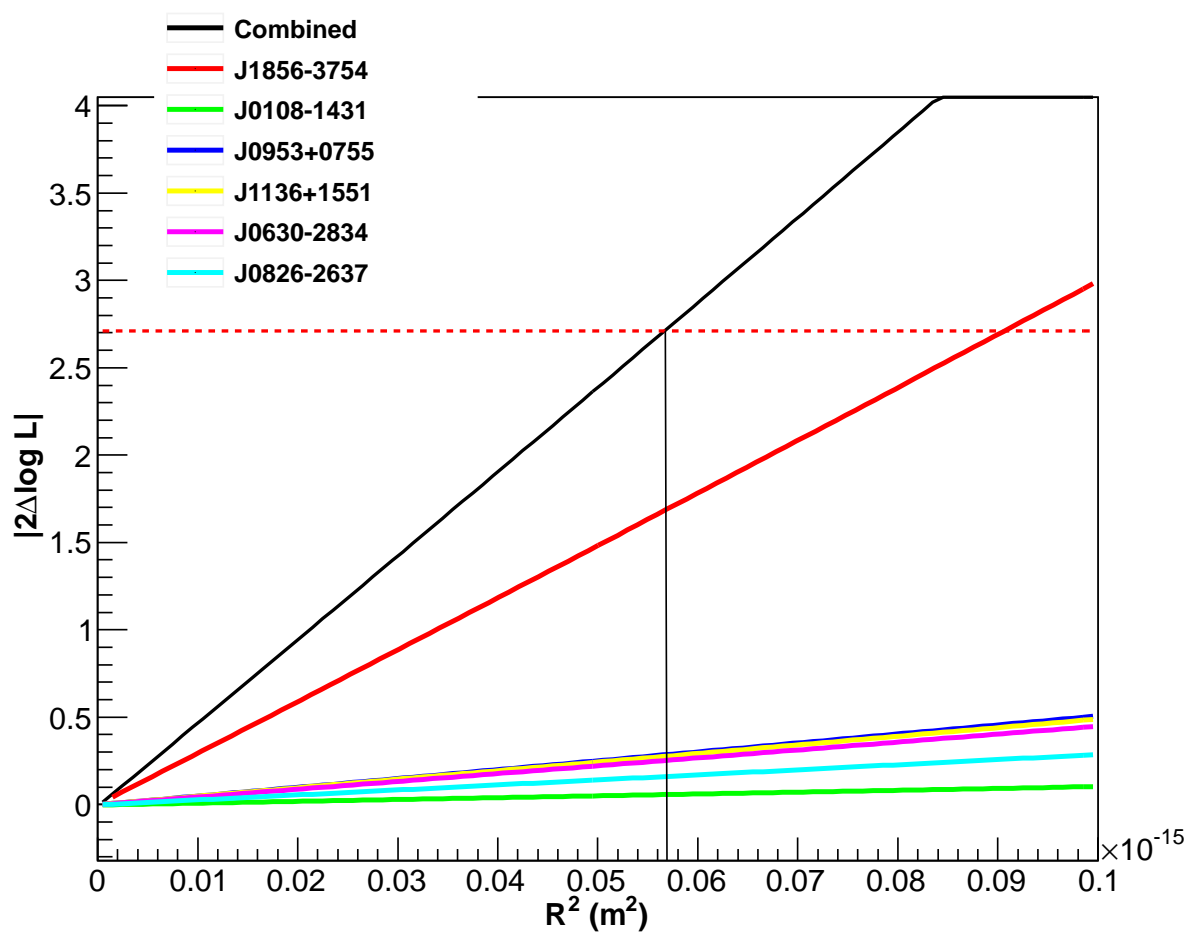

(a) $n=2$

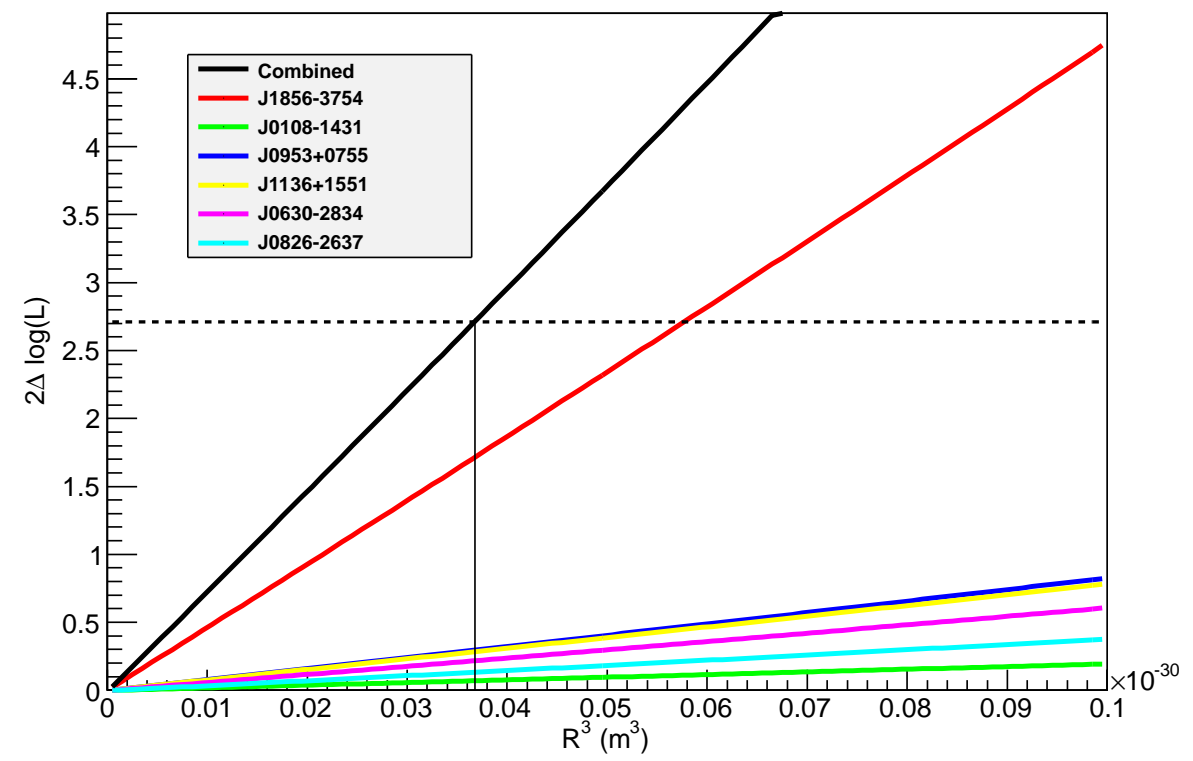

(b) $n=3$

Figure 6.9: Log-likelihood curves, for each source, as well as the summed log-likelihood error curves, for $n=2,3$. The $x$-axis corresponds to $R^{n}$, while the $y$-axis corresponds to $|2 \Delta \log \mathcal{L}|$. 


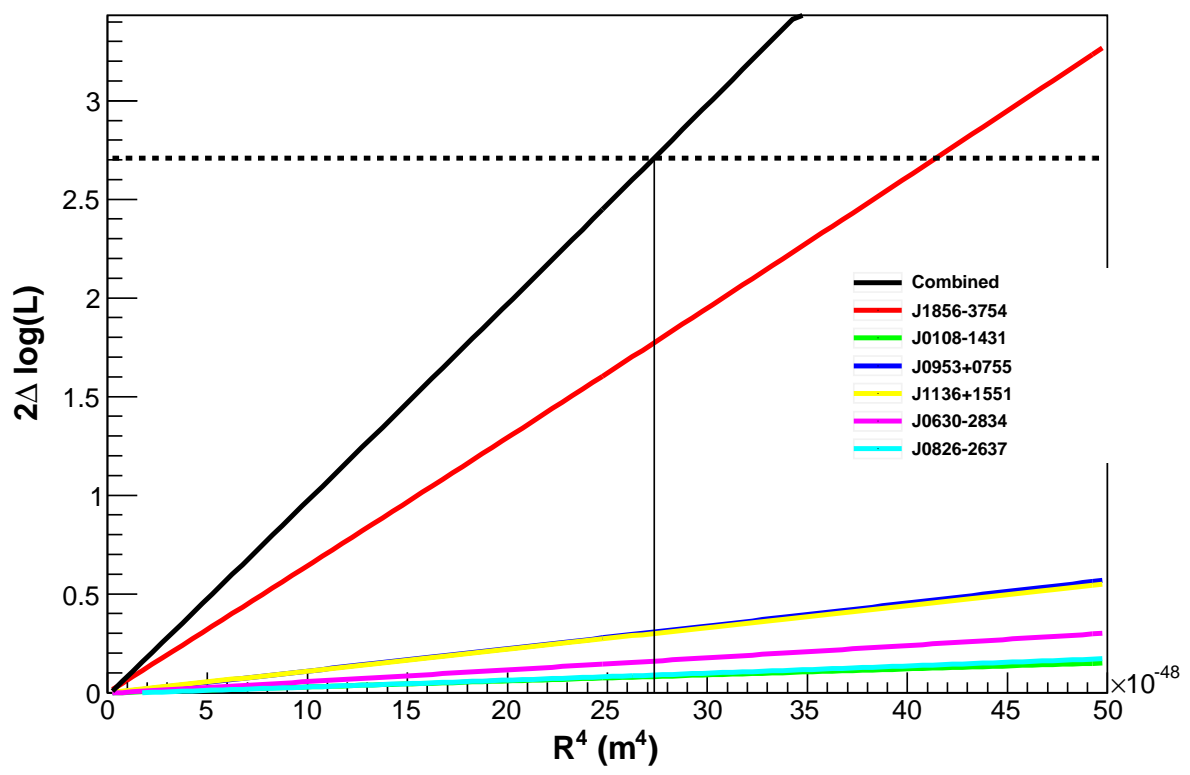

(a) $n=4$

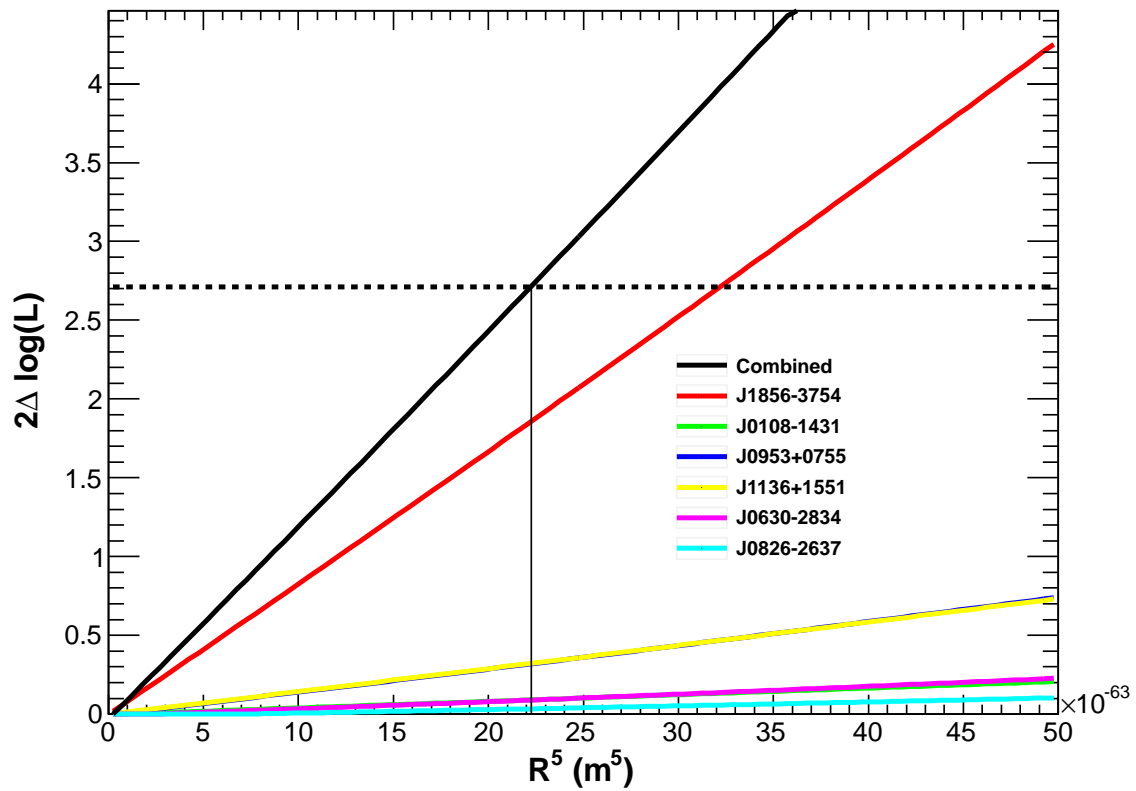

(b) $n=5$

Figure 6.10: Log-likelihood curves, for each source, as well as the summed loglikelihood error curves, for $n=4,5$. The $x$-axis corresponds to $R^{n}$, while the $y$-axis corresponds to $|2 \Delta \log \mathcal{L}|$. 


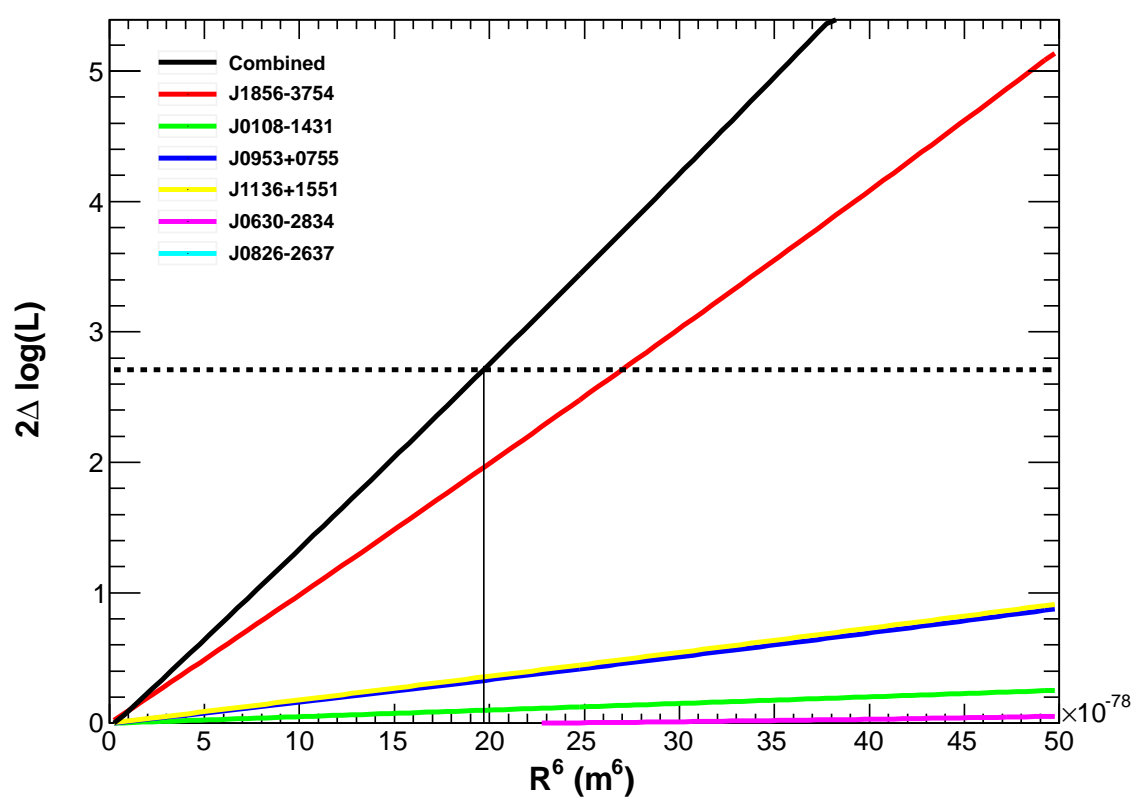

(a) $n=6$

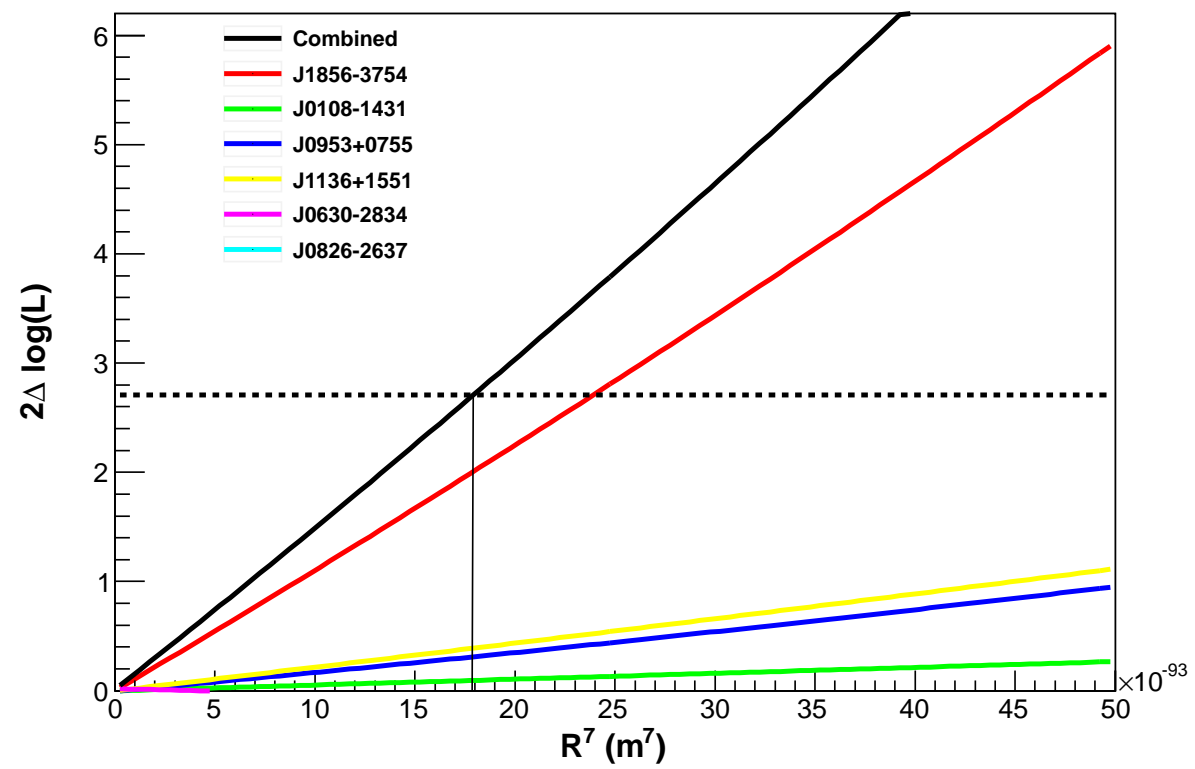

(b) $n=7$

Figure 6.11: Log-likelihood curves, for each source, as well as the summed loglikelihood error curves, for $n=6,7$. The $x$-axis corresponds to $R^{n}$, while the $y$-axis corresponds to $|2 \Delta \log \mathcal{L}|$. 


\begin{tabular}{|l|l|l|l|l|l|l|l|}
\hline $\mathrm{n}$ & Combined & HR2003 & CDF & D & LEP & ATLAS & CMS \\
\hline 2 & 250 & 97 & 2.09 & 1.40 & 1.60 & 1.5 & 3.2 \\
3 & 17 & 8.0 & 1.94 & 1.15 & 1.20 & 1.1 & 3.3 \\
4 & 2.6 & 1.5 & 1.62 & 1.04 & 0.94 & 1.8 & 3.4 \\
5 & 0.70 & 0.43 & 1.46 & 0.98 & 0.77 & 2.0 & 3.4 \\
6 & 0.25 & 0.17 & 1.36 & 0.94 & 0.66 & 2.0 & 3.4 \\
7 & 0.12 & 0.083 & 1.29 & - & - & - & - \\
\hline
\end{tabular}

Table 6.6: Comparison of 95\% CL lower limits on $M_{D}(\mathrm{TeV})$ with previous astrophysical limits and collider limits. HR2003 refers to the 2003 article by Hannestad and Raffelt [28]. Combined limits are obtained in this dissertation. Collider limits are taken from references [17, 69, 70, 71]. ATLAS and CMS results are quoted where $\Lambda / M_{D}=1$, as discussed in the Introduction chapter. ATLAS results are quoted with $3.1 \mathrm{pb}^{-1}$ of data; CMS results are quoted with $36 \mathrm{pb}^{-1}$ of data.

\subsection{Interpretation}

In this work, no evidence of KK graviton signal from neutron star spectral analysis of Fermi-LAT data was detected: the TS for each source was consistent with zero, as well as the TS from sum of the composite likelihood of the sources. However, stringent limits were placed on the compactification radius $R$ of the extra dimensions and the LED-Planck scale $M_{D}$. The limits on the $R$ are better than previously reported astrophysical limits shown in Table 6.5. Values of $M_{D}$ are shown in Table 6.6. For $n=2,3$, the values of $M_{D}$ are at least one order of magnitude larger than $1 \mathrm{TeV}$. A simple interpretation of the results rules out $n=2$ and $n=3$. In the case of flat LED, based on lower limits on $M_{D}$, we find that $n \geq 4$ extra dimensions are favored. Refer to the Conclusions chapter for further discussion. For $n=2$, these limits on LED are better than the most sensitive short distance ISL experiments. For $n=2,3$, the limits on LED are better than any past or current collider constraints. For $n=4$, the results are comparable to those of CMS's recent limits. 


\section{Chapter 7}

\section{Validation Studies}

\subsection{Verifying Upper Limits Using Different Tech- nique}

For source RXJ1856-3754, upper limits were determined from a different technique, profile likelihood, as discussed in Section [5.5. The results, shown in Table 17.1] generally agrees with the limits obtained from MINOS errors, discussed in the previous chapter, within $5 \%$.

\begin{tabular}{|l|l|l|}
\hline $\mathrm{n}$ & MINOS & UpperLimits \\
\hline 2 & $3.83 \mathrm{E}-9$ & $3.73 \mathrm{E}-9$ \\
\hline 3 & $4.00 \mathrm{E}-9$ & $3.78 \mathrm{E}-9$ \\
\hline 4 & $3.72 \mathrm{E}-9$ & $3.90 \mathrm{E}-9$ \\
\hline 5 & $4.00 \mathrm{E}-9$ & $4.01 \mathrm{E}-9$ \\
\hline 6 & $4.14 \mathrm{E}-9$ & $4.16 \mathrm{E}-9$ \\
\hline 7 & $4.19 \mathrm{E}-9$ & $4.38 \mathrm{E}-9$ \\
\hline
\end{tabular}

Table 7.1: 95\% CL upper limits $\left(\mathrm{cm}^{-2} \mathrm{~s}^{-1}\right)$ on flux for RXJ1856-3754, obtained by UpperLimits implementation of the profile likelihood method, compared with MINOS. 


\begin{tabular}{|l|l|l|}
\hline $\mathrm{n}$ & flux UL & $\begin{array}{l}\text { mean of flux UL distribution } \\
\text { with systematic uncertainties } \\
\left(1 \mathrm{E}-9 \mathrm{~cm}^{-2} \mathrm{~s}^{-1}\right)\end{array}$ \\
\hline 2 & 3.83 & $3.73_{-0.28}^{+0.44}$ \\
\hline 3 & 4.00 & $3.75_{-0.33}^{+0.47}$ \\
\hline 4 & 3.72 & $3.83_{-0.35}^{+0.62}$ \\
\hline 5 & 4.00 & $3.93_{-0.44}^{+0.73}$ \\
\hline 6 & 4.14 & $4.06_{-0.52}^{+0.92}$ \\
\hline 7 & 4.19 & $4.26_{-0.62}^{+1.2}$ \\
\hline
\end{tabular}

Table 7.2: Nominal 95\% CL flux upper limit values, compared with the mean and systematic errors of upper limit values determined from a study of varying the parameters of the background point sources and diffuse parameters. The mean and systematic errors have been obtained from Figures [7.1, 17.2, 7.3,

\subsection{Systematic Errors from 1FGL Parameters and Diffuse Parameters}

The 1FGL catalog sources have uncertainties in their normalizations and spectral indices. A study is performed in which these parameters are allowed to vary, with a Gaussian distribution: the means correspond to the 1FGL fit values, and the sigmas correspond to the errors. In addition, the fitted parameters related to the normalizations of the diffuse have associated errors; the errors in these parameters were allowed to vary as well. When including the uncertainty in the diffuse parameter in addition to the background point sources, the rms of the distribution tends to increase. The histograms of the 95\% CL flux upper limit values from the distributions are shown in Figures 7.1, 7.2, 7.3, and the mean and systematic uncertainties corresponding to the distributions are shown in Table 17.2. The histograms have been fit to a sum of three Gaussian functions, on account of the long tails on the flux upper limits . 


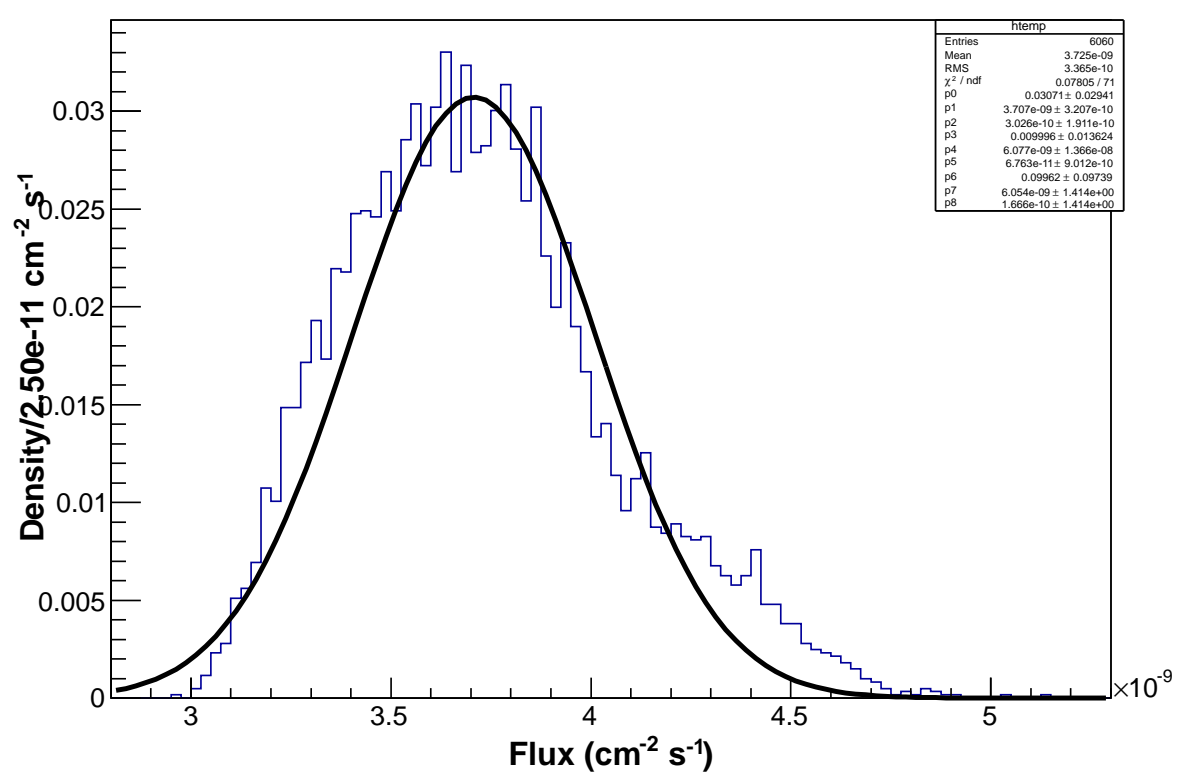

(a) $n=2$

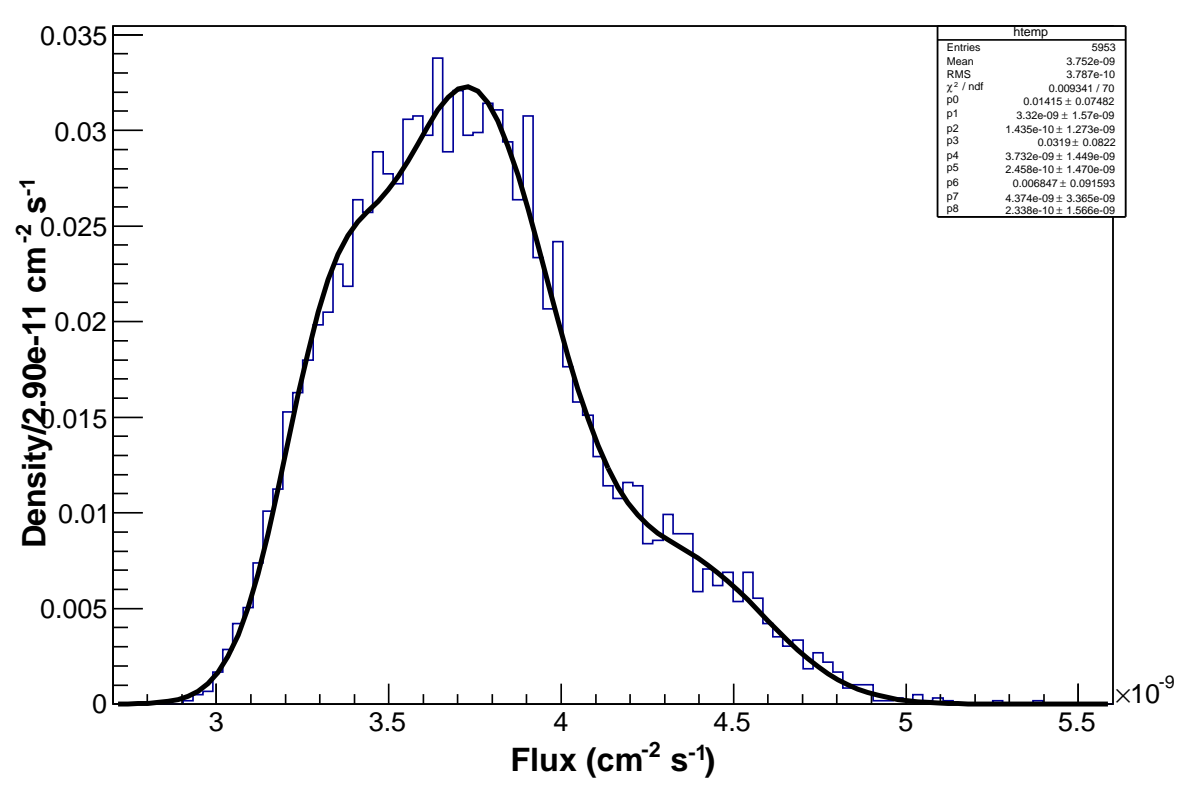

(b) $n=3$

Figure 7.1: Histograms of the 95\% CL flux upper limit distributions of RX J18563754 , for $n=2,3$. Each of the spectral parameters of the background point sources, as well as the diffuse parameters, have been varied according to a normal distribution, in a MC simulation. The histograms have been fit to a triple Gaussian function, due to the long tails of the distributions. 


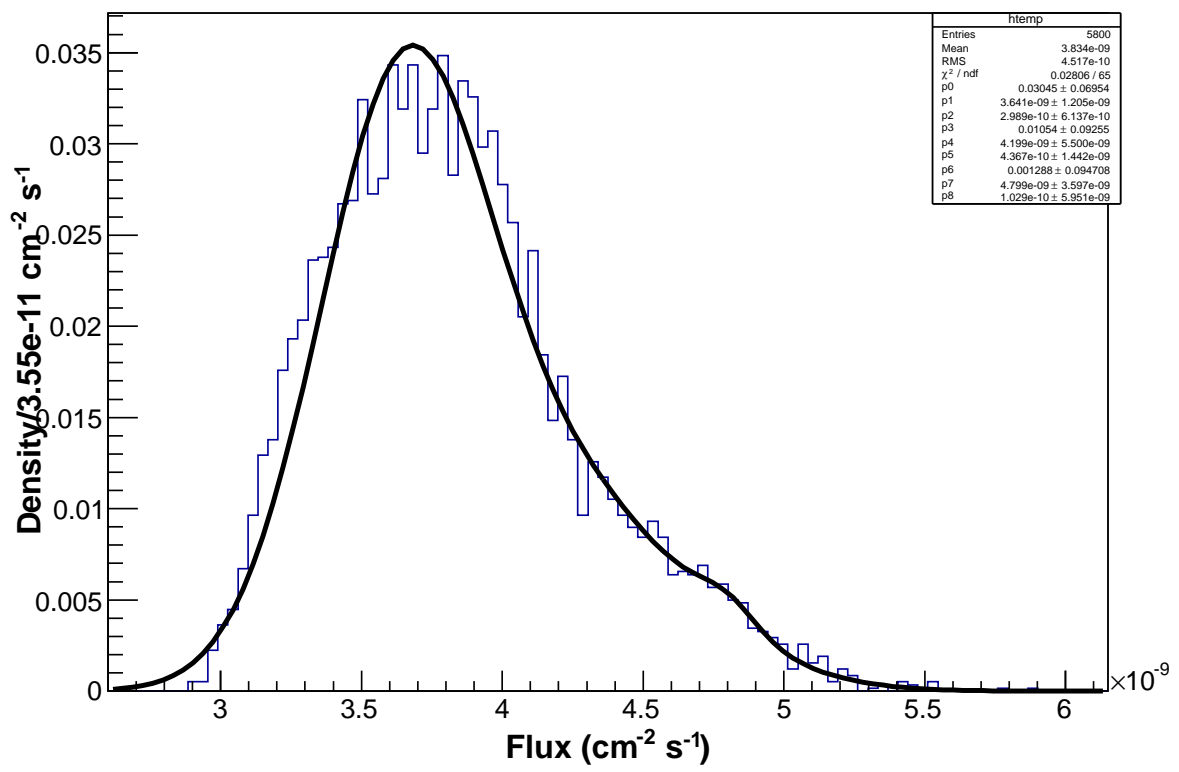

(a) $n=4$

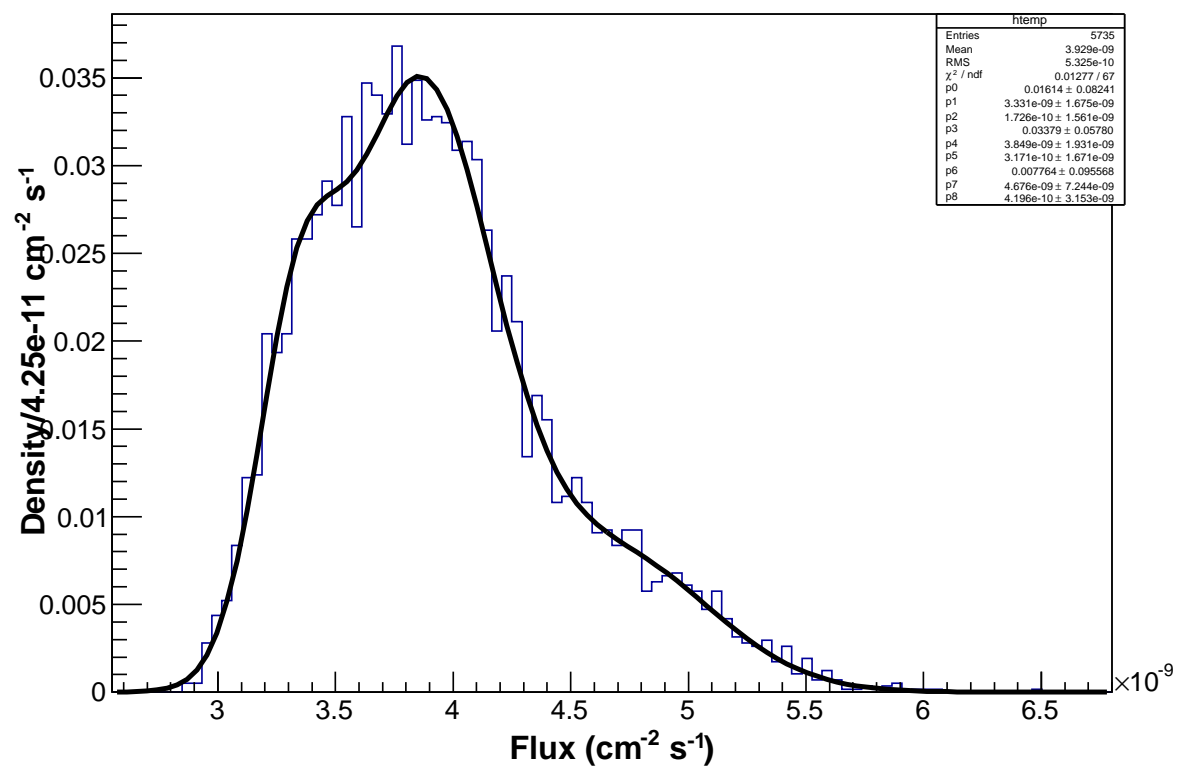

(b) $n=5$

Figure 7.2: Histograms of the 95\% CL flux upper limit distributions of RX J18563754 , for $n=4,5$. Each of the spectral parameters of the background point sources, as well as the diffuse parameters, have been varied according to a normal distribution, in a MC simulation. The histograms have been fit to a triple Gaussian function, due to the long tails of the distributions. 


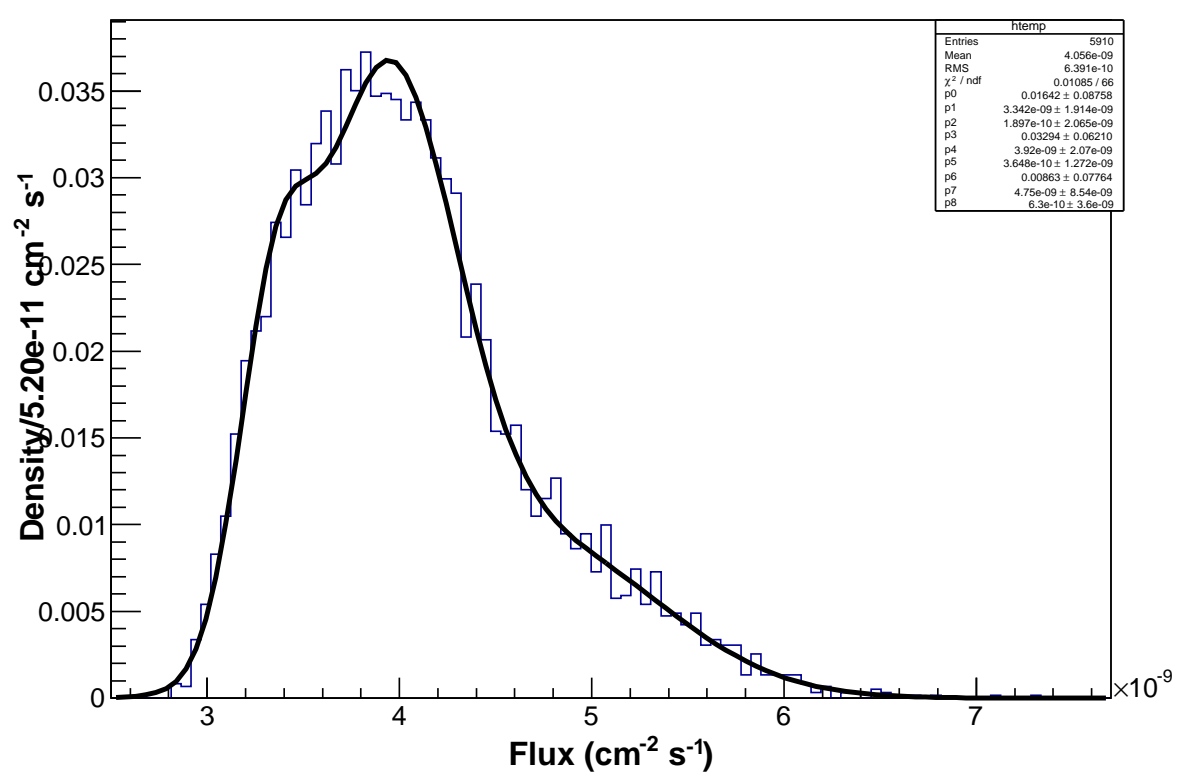

(a) $n=6$

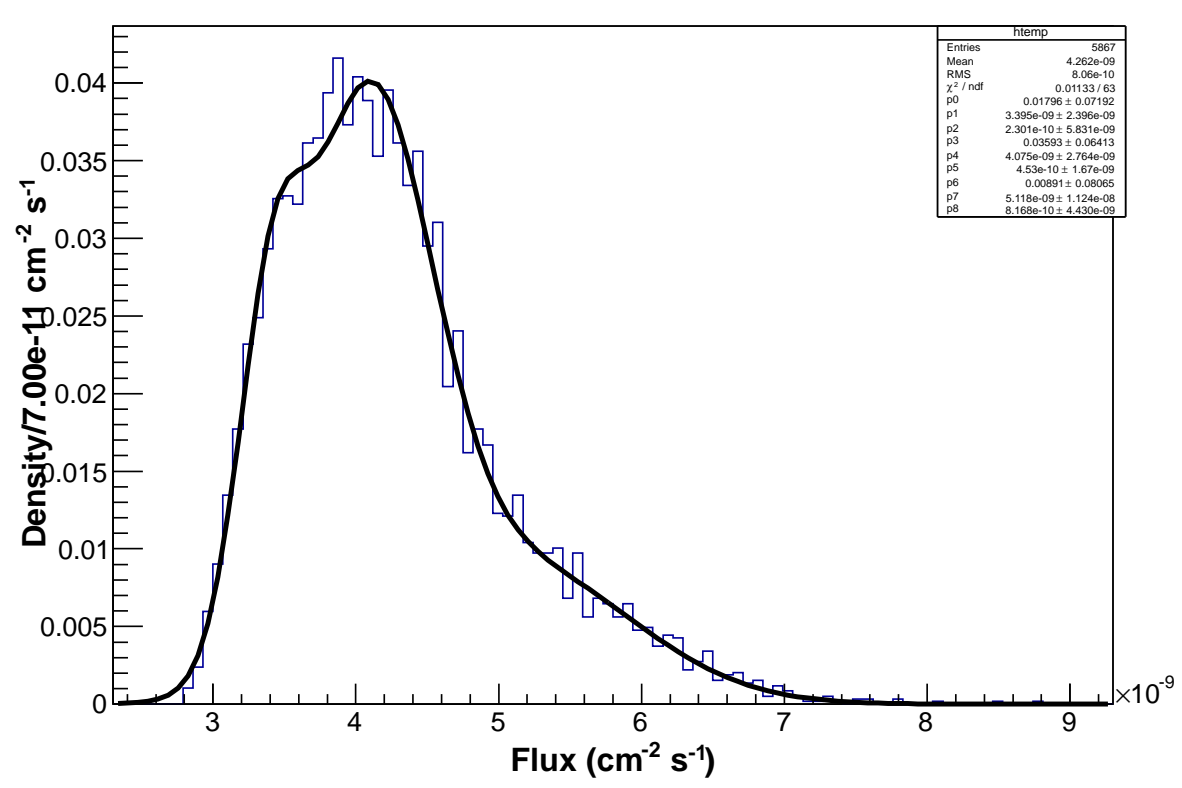

(b) $n=7$

Figure 7.3: Histograms of the 95\% CL flux upper limit distributions of RX J18563754 , for $n=6,7$. Each of the spectral parameters of the background point sources, as well as the diffuse parameters, have been varied according to a normal distribution, in a MC simulation. The histograms have been fit to a triple Gaussian function, due to the long tails of the distributions. 


\begin{tabular}{|l|l|}
\hline $\mathrm{n}$ & Flux UL $\left(\mathrm{cm}^{-2} \mathrm{~s}^{-1}\right)$ \\
\hline 2 & $3.9 \mathrm{E}-9$ \\
\hline 3 & $3.4 \mathrm{E}-9$ \\
\hline 4 & $2.9 \mathrm{E}-9$ \\
\hline 5 & $2.6 \mathrm{E}-9$ \\
\hline 6 & $2.2 \mathrm{E}-9$ \\
\hline 7 & $1.9 \mathrm{E}-9$ \\
\hline
\end{tabular}

Table 7.3: Flux upper limit values, at 97.5\% CL, computed according to MINOS errors, for source RX J1856-3754.

\subsection{Simulations of sources within the ROI for RXJ1856- 3754}

To check the validity of the upper limits for the source yielding the best LED limits, a study was done to simulate sources within the ROI corresponding to RXJ1856-3754. No putative neutron star source with a KK graviton decay spectrum was placed at the center. However, within the ROI, background point sources within a $7.2^{\circ}$ radius of RXJ1856-3754 were modeled as power law spectra; this choice of radius was determined by approximately twice the PSF radius of $4.7^{\circ}$ at $100 \mathrm{MeV}$. The sources included were standard diffuse models as used in the analysis. A one-year duration simulated FT2 file was used. The program used to generate the photon events FT1 file was gtobssim, a ScienceTools utility. A flux of $3.9 \times 10^{-9} \mathrm{~cm}^{-2} \mathrm{~s}^{-1}$ was determined as the $97.5 \%$ CL upper limit from MINOS errors for $n=2$, and generally decreases with increasing $n$, as shown in Table 7.3. This simulation demonstrates that the flux upper limit derived from the analysis is a conservative one. It is likely that modeling all point sources within $14^{\circ}$ source radius would increase the upper limit. The simulation time when adding new sources increases, however, and becomes a prohibitive factor when running the simulations. 


\begin{tabular}{|l|l|l|l|l|}
\hline $\mathrm{n}$ & $R(\mathrm{~m})\left(\mathrm{P} 6 \_V 3\right)$ & flux UL (P6_V3) & $R(\mathrm{~m})\left(\mathrm{P} 6 \_V 11\right)$ & flux UL (P6_V11) \\
\hline 2 & $9.52 \mathrm{E}-9$ & $3.83 \mathrm{E}-09$ & $9.88 \mathrm{E}-09$ & $4.11 \mathrm{E}-09$ \\
3 & $3.89 \mathrm{E}-11$ & $4.00 \mathrm{E}-09$ & $3.98 \mathrm{E}-11$ & $4.21 \mathrm{E}-09$ \\
4 & $2.54 \mathrm{E}-12$ & $3.72 \mathrm{E}-09$ & $2.61 \mathrm{E}-12$ & $4.95 \mathrm{E}-09$ \\
5 & $5.03 \mathrm{E}-13$ & $4.00 \mathrm{E}-09$ & $5.17 \mathrm{E}-13$ & $4.62 \mathrm{E}-09$ \\
6 & $1.73 \mathrm{E}-13$ & $4.14 \mathrm{E}-09$ & $1.78 \mathrm{E}-13$ & $4.92 \mathrm{E}-09$ \\
7 & $8.15 \mathrm{E}-14$ & $4.19 \mathrm{E}-09$ & $8.38 \mathrm{E}-14$ & $5.32 \mathrm{E}-09$ \\
\hline
\end{tabular}

Table 7.4: 95\% CL upper limits on $R(\mathrm{~m})$ and fluxes for RXJ1856-3754, compared in 2 different P6 IRFs.

\subsection{Using an Updated IRF}

An updated IRF, P6_V11_DIFFUSE, was developed by the collaboration to take into account in-flight calibrations with known sources: Vela from $100 \mathrm{MeV}$ to $10 \mathrm{GeV}$, and AGNs from $10 \mathrm{GeV}$ to $100 \mathrm{GeV}[72]$. New templates for the diffuse models were developed corresponding to this IRF, gll_iem_v02_P6V11.fit and isotropic_iem_v02.txt to be compatible with this new IRF. Performance comparisons of this IRF with P6_V3_DIFFUSE are shown in Figure 7.4. It may be observed that the PSF is larger for this IRF, as shown. Limits on LED for source RXJ1856-3754 were determined as shown in Table 17.4. The limits are generally worse, likely due to the larger PSF. Although it could be an advantage to use a more accurate IRF, the problem lies with consistency with 1FGL point sources, whose spectral behavior has been modeled using P6_V3_DIFFUSE. 


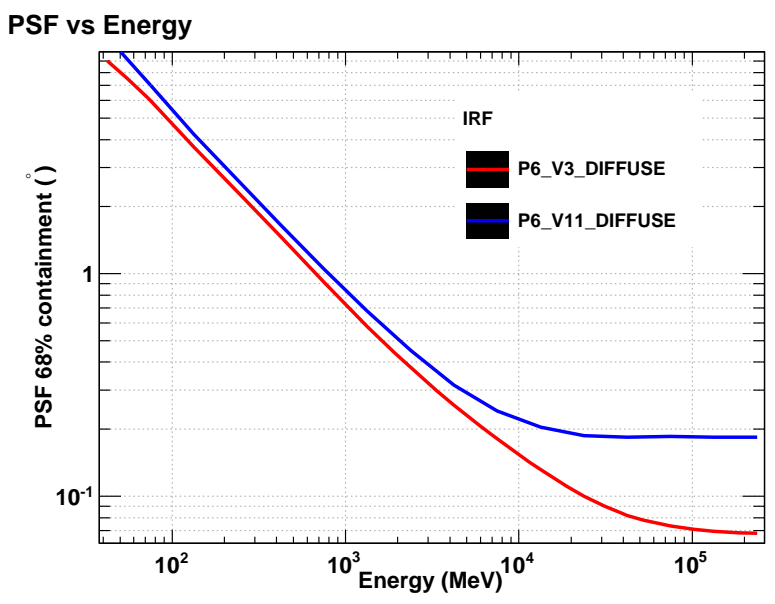

(a) PSF Comparison

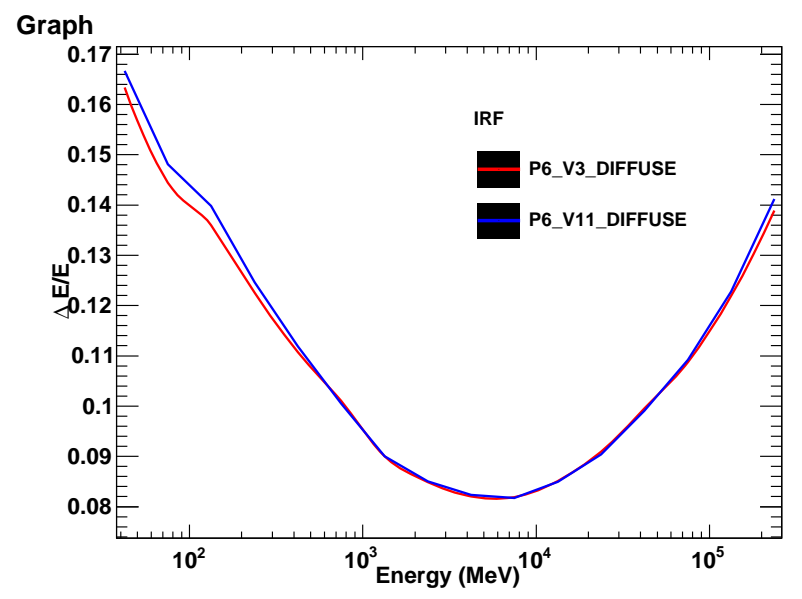

(b) Energy Resolution Comparison

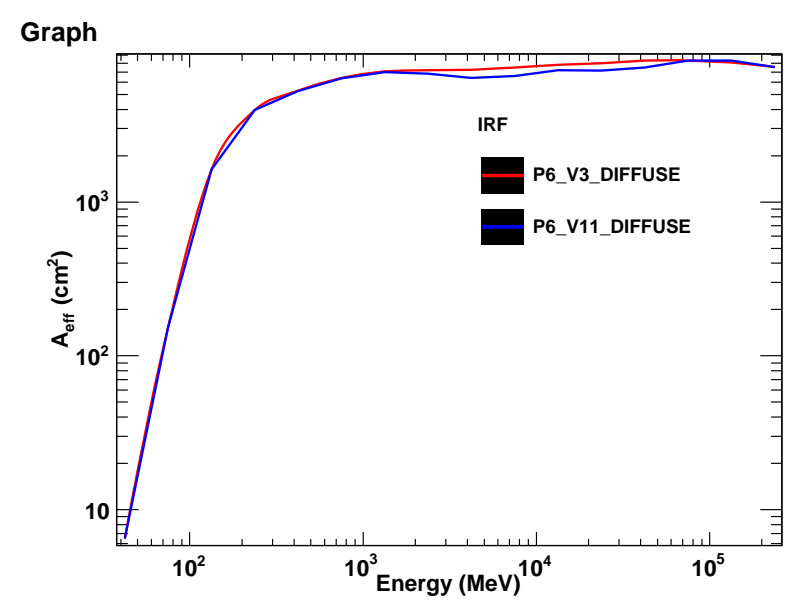

(c) Effective Area Comparison

Figure 7.4: Performance comparisons of 2 Pass 6 IRFs considered in this chapter, P6_V3_DIFFUSE and P6_V11_DIFFUSE. 


\section{Chapter 8}

\section{Conclusions}

\subsection{Interpretation}

\subsection{Generalization of LED Bounds}

In this dissertation, stringent limits were placed on the compactification radius $R$ of the extra dimensions and the LED-Planck scale $M_{D}$. In the case of flat LED, based on lower limits on $M_{D}$, we find that $n \geq 4$ extra dimensions are favored. This favors certain models of supersymmetry and supergravity. For $n=2,3$, the interpretation of the results suggests that if unification is to occur at the $\mathrm{TeV}$ scale, there must be a more complicated topology of the extra dimensions than toroidal compactification. For instance, this may occur when some of the extra dimensions have different sizes [9].

\subsubsection{Weakly-warped Extra Dimensions}

Weakly-warped extra dimensions scenarios, with only one or two additional space dimensions, as discussed in the introduction, is a theoretical possibility [21]. Predictions for $n=2$ and different values for $k$ can be calculated. 


\subsubsection{Axion-Like Particles}

These bounds on LED may be able to be translated in bounds on emission of axion like particles (ALPs). Instead of the coupling constant related to the Planck mass, the relevant coupling constant would be the axion coupling constant $\left(f_{a}\right)$, which would be related in the LED theoretical paradigm as 10]:

$$
\frac{1}{f_{a}^{2}} \rightarrow \frac{T^{n}}{M_{D}^{n+2}}
$$

A detailed study on a way to set bounds based on gamma-ray data with FermiLAT has been published: the authors excluded the ALP mass between $50 \mathrm{keV}$ and 1 $\mathrm{GeV}$ based on point-source sensitivity of the LAT[73].

\subsection{Possible Future Improvements}

More accurate modeling of the physical processes leading to the spectral energy distribution and expected fluxes is an area that can be improved, as discussed in Section 8.3.1. Improvements in measurements with the Fermi-LAT is another area where the analysis could be improved, as will be discussed in Section 8.3.2.

\subsubsection{Theoretical Improvements}

There are several theoretical parameters whose uncertainty might affect the spectra for the pulsars: supernova core temperature, timescale of core collapse, fraction of gravitational collapse energy emitted into KK gravitons. Neutron star parameters include mass and radius of the neutron star, which may differ from those values assumed in this dissertation.

\section{Temperature Dependence}

The limits on LED are a strong function of temperature. The dependence enters through two effects: changing the constant $k_{n}$ and changing the distribution of gamma-ray energies. The limits are affected, since $k_{n} \sim T^{-n-5.5}$; in other words, 


\begin{tabular}{|l|l|l|l|}
\hline$n$ & $T=15 \mathrm{MeV}$ & $T=30 \mathrm{MeV}$ & $T=45 \mathrm{MeV}$ \\
\hline 2 & $3.409 \mathrm{E} 4$ & $6.171 \mathrm{E} 6$ & $1.291 \mathrm{E} 8$ \\
\hline 3 & $1.455 \mathrm{E} 19$ & $5.269 \mathrm{E} 21$ & $1.654 \mathrm{E} 23$ \\
\hline 4 & $6.380 \mathrm{E} 33$ & $4.619 \mathrm{E} 36$ & $2.175 \mathrm{E} 38$ \\
\hline 5 & $2.941 \mathrm{E} 48$ & $4.259 \mathrm{E} 51$ & $3.008 \mathrm{E} 53$ \\
\hline 6 & $1.433 \mathrm{E} 63$ & $4.151 \mathrm{E} 66$ & $4.397 \mathrm{E} 68$ \\
\hline 7 & $7.376 \mathrm{E} 77$ & $4.273 \mathrm{E} 81$ & $6.790 \mathrm{E} 83$ \\
\hline
\end{tabular}

Table 8.1: Table of values of $k_{n}$, evaluated at different temperatures.

by modifying $k_{n}$, the bounds on LED size improve as $R \sim\left(\frac{T}{30 \mathrm{MeV}}\right)^{-1-5.5 / n}$. Values of $k_{n}$, evaluated at $T=15,30,45 \mathrm{MeV}$, are shown in Table 8.1] In addition, for higher temperatures, the distribution of energies is shifted to higher gamma-ray energies. Quantitatively, this increases the integral of the distribution function above $100 \mathrm{MeV}, \int_{100 \mathrm{MeV}}^{\infty} d N / d E d E$, which tends to improve the bounds. Sample curves are shown in Figure 8.1. Limits placed on $R$ from source RX J1856-3754 may vary by a few orders of magnitude, as shown in Table 8.2. It may be noted, however, that the our limits are rather conservative, since $T=30 \mathrm{MeV}$ is a reasonable lower bound on the SN core temperature [29].

\section{Other Model Parameters}

By varying the timescale of core collapse, $\Delta t_{N S}$, the limits on $R$ vary as $\left(\Delta t_{N S}\right)^{-1 / n}$. Estimates for this parameter vary from $5 \mathrm{~s}$ to $20 \mathrm{~s}[35]$. Varying the fraction of energy lost into $f_{K K}$, the limits on $R$ vary as $f_{K K}^{-1 / n}$. Thus, we see that the limits depend weakly on variations of these two parameters.

Although the mass of neutron stars is approximately given by the Chandrasekhar mass, $M_{N S}=1.4 M_{\odot}$, it is not an accurately known parameter, when the neutron star is not part of a binary system.

\section{Other Assumptions}

A more accurate knowledge of the process of graviton emission in nucleon-nucleon bremsstrahlung could also be considered, leading to a more accurate spectrum of 


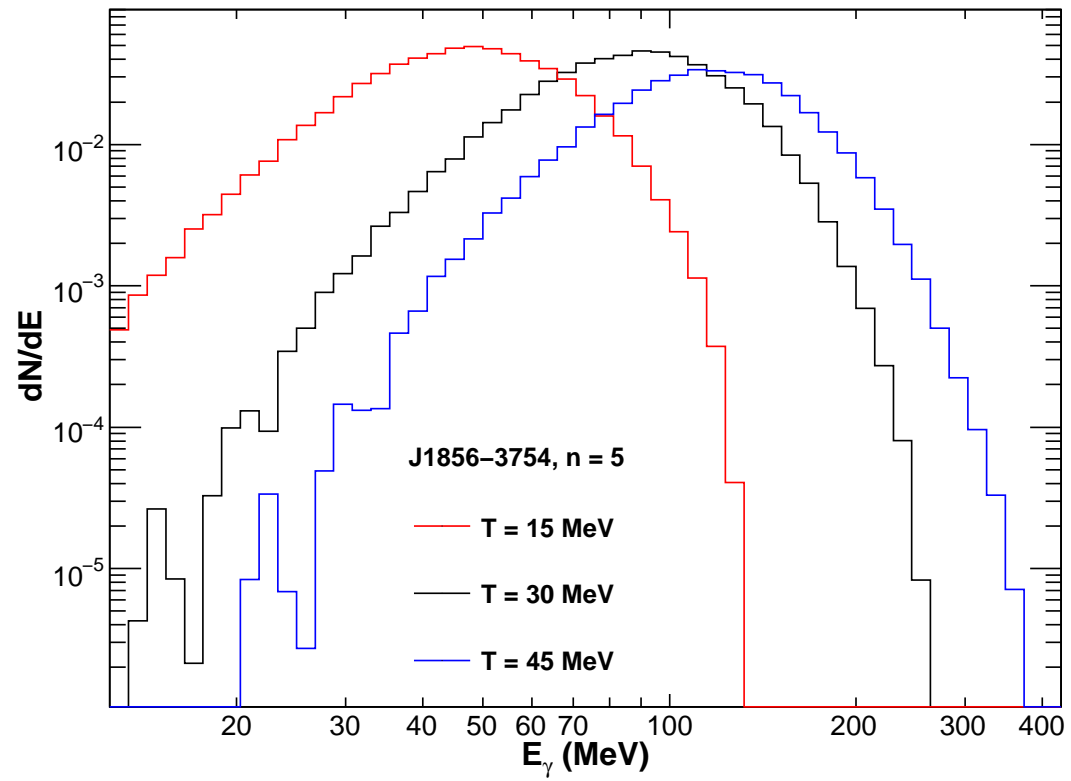

Figure 8.1: Spectra $d N_{n} / d E_{\gamma}$, at different temperatures, for $n=5$.

\begin{tabular}{|l|l|l|l|}
\hline $\mathrm{n}$ & $T=15 \mathrm{MeV}$ & $T=30 \mathrm{MeV}$ & $T=45 \mathrm{MeV}$ \\
\hline 2 & $8.4 \mathrm{E}-6$ & $9.5 \mathrm{E}-9$ & $1.2 \mathrm{E}-9$ \\
\hline 3 & $1.7 \mathrm{E}-9$ & $3.9 \mathrm{E}-11$ & $9.3 \mathrm{E}-12$ \\
\hline 4 & $4.0 \mathrm{E}-11$ & $2.5 \mathrm{E}-12$ & $8.5 \mathrm{E}-13$ \\
\hline 5 & $4.8 \mathrm{E}-12$ & $5.0 \mathrm{E}-13$ & $2.1 \mathrm{E}-13$ \\
\hline 6 & $3.6 \mathrm{E}-12$ & $1.7 \mathrm{E}-13$ & $8.1 \mathrm{E}-14$ \\
\hline 7 & $4.2 \mathrm{E}-13$ & $8.2 \mathrm{E}-14$ & $4.1 \mathrm{E}-14$ \\
\hline
\end{tabular}

Table 8.2: Upper limits on $R(\mathrm{~m})$, evaluated for different SN core temperatures, for source RX J1856-3754. 
graviton masses.

It is known that an accurate modeling of the neutron star gravitational potential involves invoking general relativity, and using a correct space-time metric, such as the Schwarzchild metric. Thus radial orbits dependence on $\mu$ would be different. Also. the photons would be red-shifted.

Improvements to modeling the graviton decay signal could also be implemented. Erber's expressions are only approximately correct near the neutron star surface. A more accurate treatment might include a consideration of the Landau levels, as implemented by Alice Harding and others: this model has been shown to be more more accurate near the neutron star surface, as well as being validated by experimental observations of pulsars [4]. The contribution to gamma ray signal from pair cascades of graviton decay electrons and positrons was not investigated in this analysis.

\section{KK Graviton Emission from SN Cores Based on Diffuse Emission}

Hannestad and Raffelt constrained $f_{K K}$ based on the EGRET measurement of the spectrum of the extragalactic diffuse background. The Fermi-LAT measurement of the diffuse, based on 1 year of survey data, is different from that determined from EGRET; it has a softer spectrum, of index -2.41, as compared with EGRET's of approximately $-2.1[75]$ [30]. This implies that below $100 \mathrm{MeV}$, the Fermi diffuse spectrum predicts a higher $f_{K K}$, based on comparison with the $n=2$ and $n=3$ contribution from past SNe to the spectrum of diffuse. This would lead to more stringent limits on extra dimensions; for $n=2$. For the $n=2$ case, the improvement to the limit on $R$ could be a factor of at least $20 \%$.

\subsubsection{Instrumental Improvements}

Improvements from the instrument could arise from consideration of events below 100 $\mathrm{MeV}$ in energy. This is especially important for the case of a low number of extra dimensions $(n \leq 4)$, which are the more interesting cases as compared with colliders.

\footnotetext{
${ }^{1}$ The integral flux for diffuse emission above $100 \mathrm{MeV}$ is $1.03 \times 10^{-5} \mathrm{~cm}^{-2} \mathrm{~s}^{-1} \mathrm{sr}^{-1}$, less than the value for EGRET's value of $\left.1.5^{-5} \mathrm{~cm}^{-2} \mathrm{~s}^{-1} \mathrm{sr}^{-1}[75], 30\right]$.
} 
It may be observed that the peak of the spectral energy distributions for $n=2$ lies between 50 and $60 \mathrm{MeV}$.

The reliable measurement of the energy his was hampered by systematic problems in Pass 6 IRFs in this range, as explored in the appendix. Pass 7, which has achieved a final use stage, does significantly better, and the next generation IRF pass 8, under development by the collaboration, will do even better. The design specification, as described in the Science Requirements document, was to measure gamma-rays above $20 \mathrm{MeV}$.

Despite possible improvements in the reconstruction of the energy, the effective area below $100 \mathrm{MeV}$ is improved, but remains low compared to the LAT optimum at $1 \mathrm{GeV}$.

\subsubsection{Data Analysis Improvements}

Various parameters that are inputs into the model, but are not of primary interest, include distance to each pulsar, have associated uncertainties. A proper treatment in a likelihood-based analysis would involve marginalization over this nuisance parameter. An implementation of this statistical method has recently been added to the capabilities of the Fermi ScienceTools. Other parameters that could be treated in such a fashion include parameters of the background point sources. Even parameters such as $\Delta t_{N S}$ and $f_{K K}$ could be treated in such a fashion.

\subsection{Considering a Population of NS in the Galactic Center}

An approach considered by the 2004 paper of Cassé, Sigl, Paul and Bertone was to average over a population of neutron stars in the galactic bulge [19]. They assumed a stellar mass function, with a power law index of -2.3 , to infer the number of neutron stars in the galactic center, $N_{N S}=4.2 \times 10^{8}$. They also took as an upper limit for the flux above $100 \mathrm{MeV}$ of $8 \times 10^{-7} \mathrm{~cm}^{-2} \mathrm{~s}^{-1}$, and distance to the stars in the galactic center at $8 \mathrm{kpc}$. 
There are a few drawbacks to this method. The number of NSs could be different than their estimate, with a different mass function. The ages and magnetic fields of the vast majority of the NS are not known. If most of the KK graviton decay gamma-rays don't get out from the NSs, their derived limits could well be gross underestimation. The distance of $8 \mathrm{kpc}$ to all the sources is an approximation, due to geometry, since the galactic center region is defined as a volume, not as a point.

\subsection{Constraining LED with Diffuse Gamma-Ray Emission}

Using gamma-ray observations of the diffuse emission, limits on LED may be set. Assuming that gravitons are produced in the early universe during with reheating, a relic graviton decay spectrum lasting to the present time may exist. This has been analyzed by Fairbairn [76]. This problem was subsequently examined by Steen Hannestad, and limits on LED were obtained[77]. He considered KK gravitons that were produced during the radiation-dominated epoch. Based on EGRET measurements of the diffuse emission, upper limits of $R<2.2 \times 10^{-8} \mathrm{~m}$ for $n=2$ and $R<2.5 \times 10^{-11} \mathrm{~m}$ for $n=3$ were obtained, which are more stringent than the limits from the Hannestad and Raffelt 2003 paper, but less stringent than the limits obtained in this dissertation for $n=2$. Reanalysis of this method is a possible area where the Fermi-LAT data can lead to more stringent constraints on LED. 
Appendices 

Appendix A

Fitted Differential Flux

Distributions for Each Neutron

Star Source 


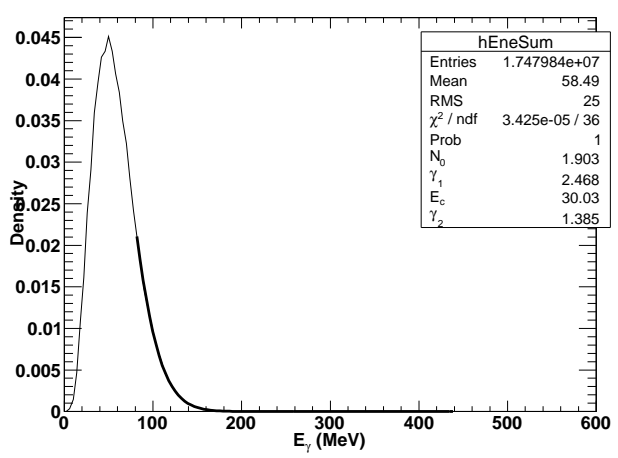

(a) $n=2$

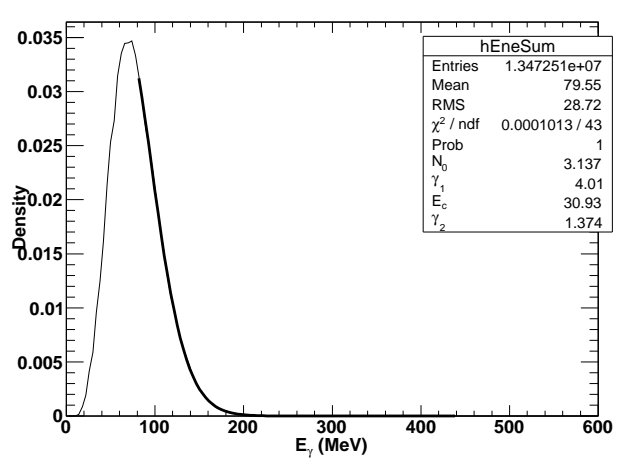

(c) $n=4$

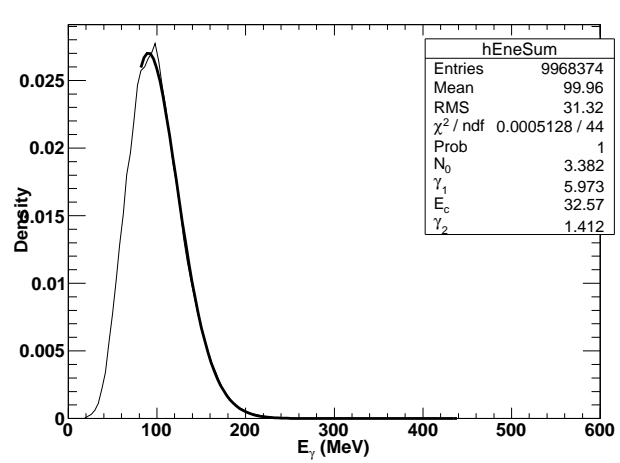

(e) $n=6$

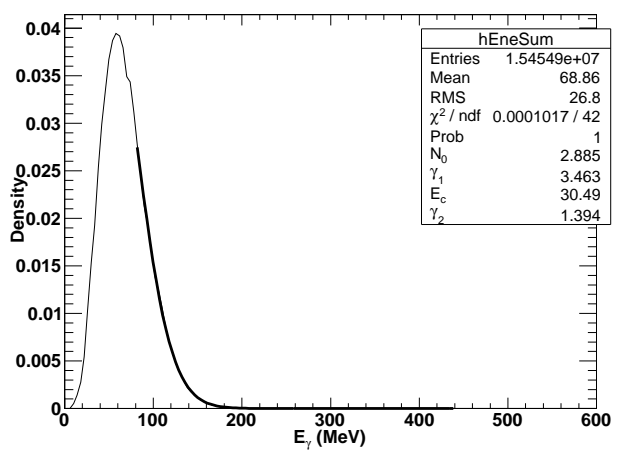

(b) $n=3$

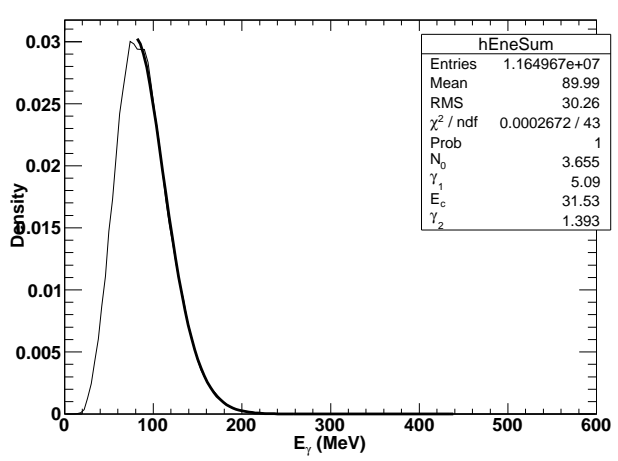

(d) $n=5$

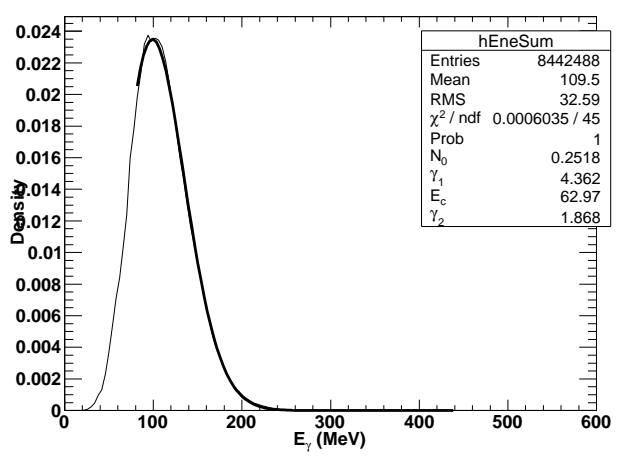

(f) $n=7$

Figure A.1: Histograms of the differential flux distributions for RXJ1856-3754, determined from Monte Carlo, for different $n$. Each histogram has been fit to a PowerLawSuperExpCutoff model, using MINUIT optimizer. 


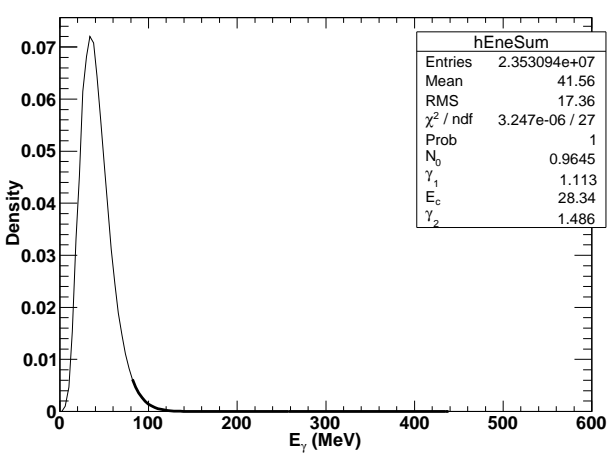

(a) $n=2$

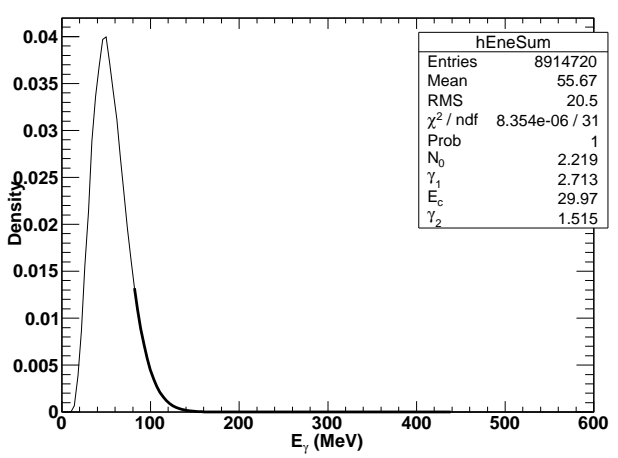

(c) $n=4$

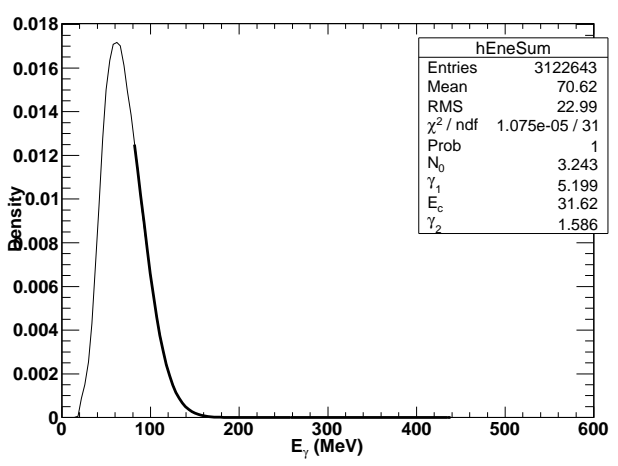

(e) $n=6$

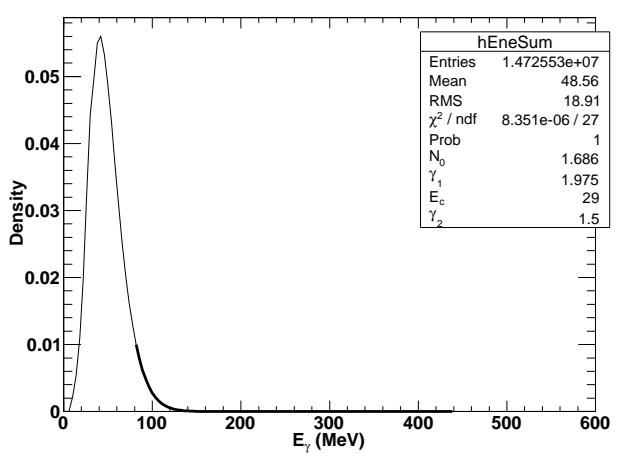

(b) $n=3$

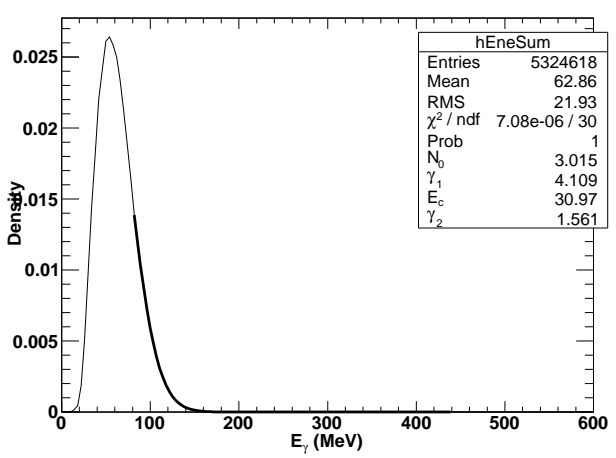

(d) $n=5$

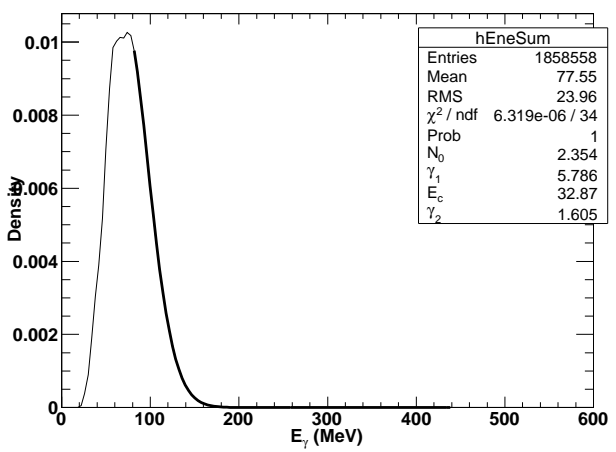

(f) $n=7$

Figure A.2: Histograms of the differential flux distributions for PSR J0108-3754, determined from Monte Carlo, for different $n$. Each histogram has been fit to a PowerLawSuperExpCutoff model, using MINUIT optimizer. 


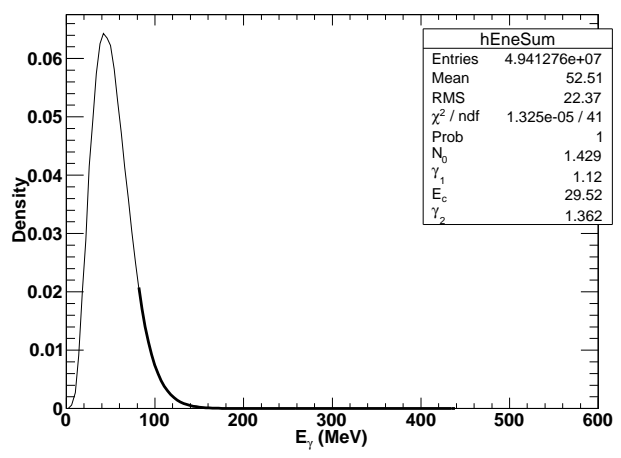

(a) $n=2$

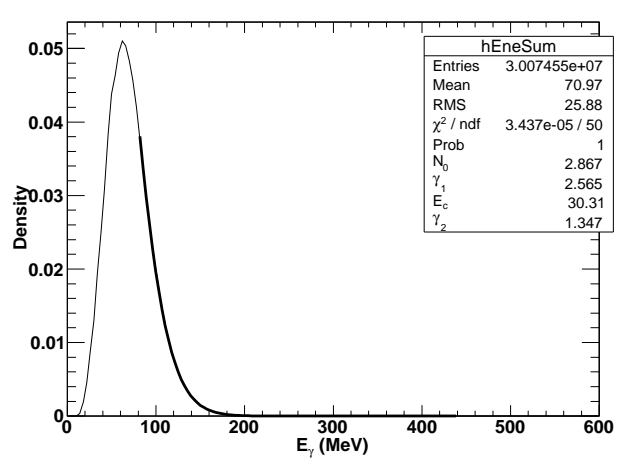

(c) $n=4$

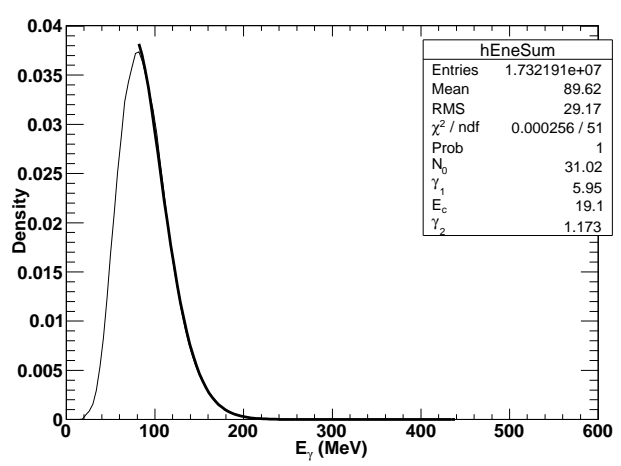

(e) $n=6$

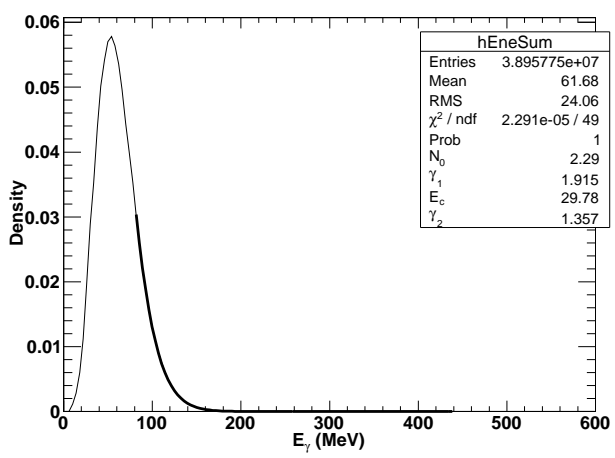

(b) $n=3$

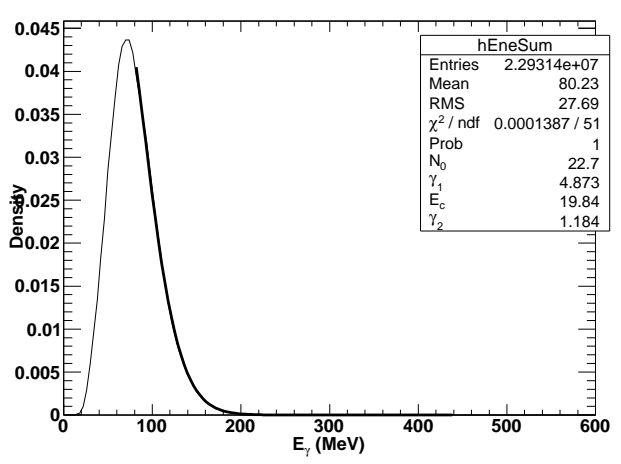

(d) $n=5$

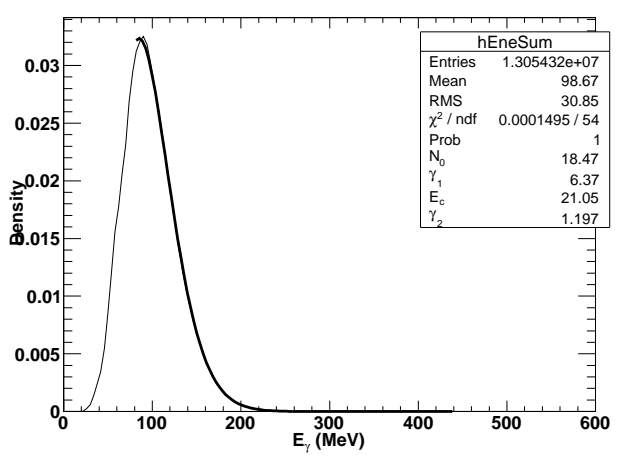

(f) $n=7$

Figure A.3: Histograms of the differential flux distributions for PSR J09053+0755, determined from Monte Carlo, for different $n$. Each histogram has been fit to a PowerLawSuperExpCutoff model, using MINUIT optimizer. 


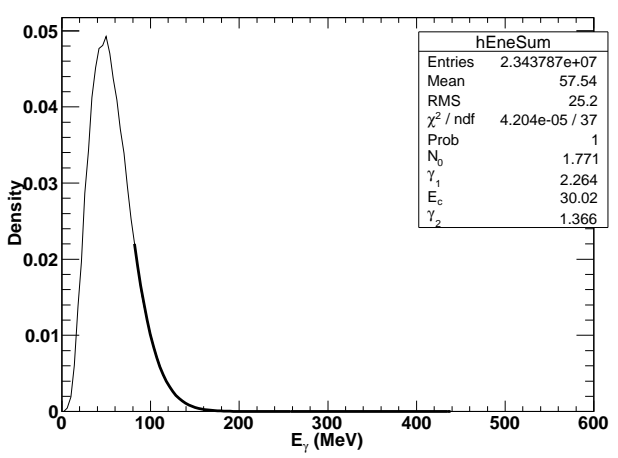

(a) $n=2$

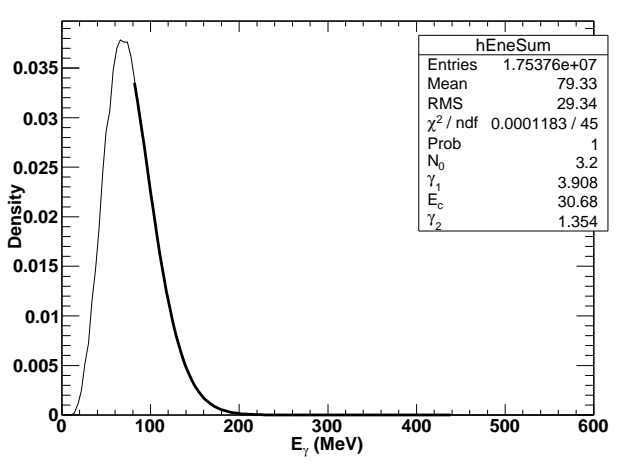

(c) $n=4$

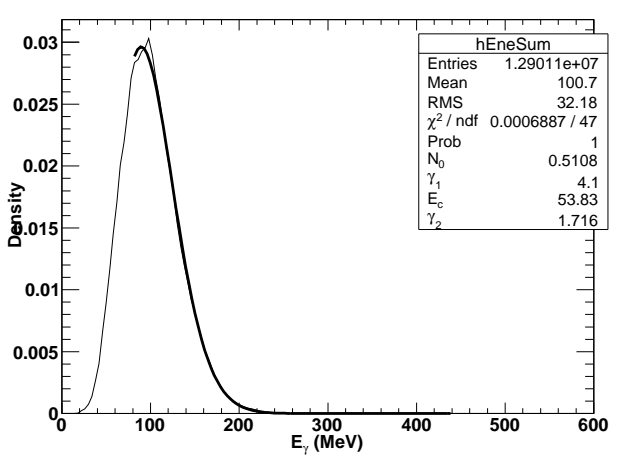

(e) $n=6$

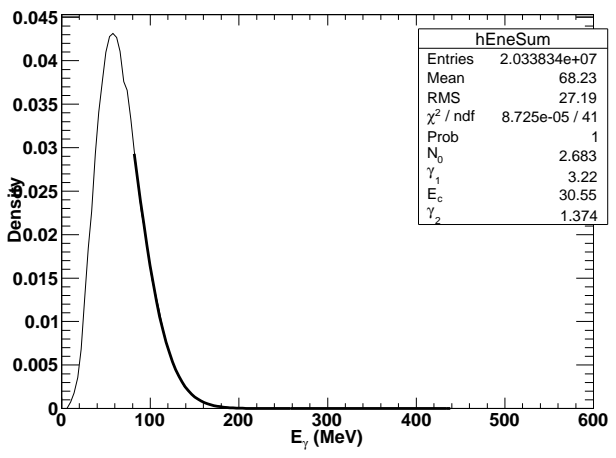

(b) $n=3$

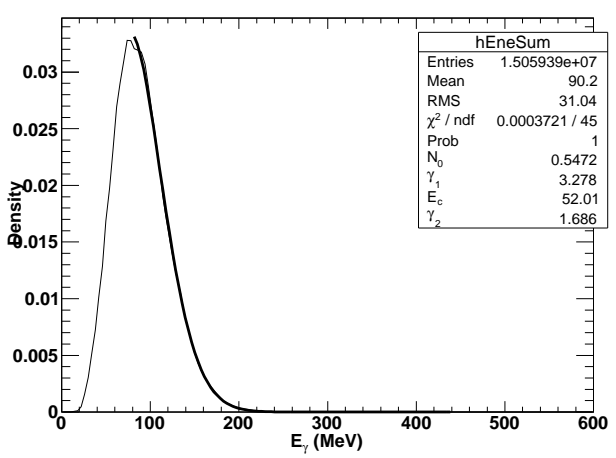

(d) $n=5$

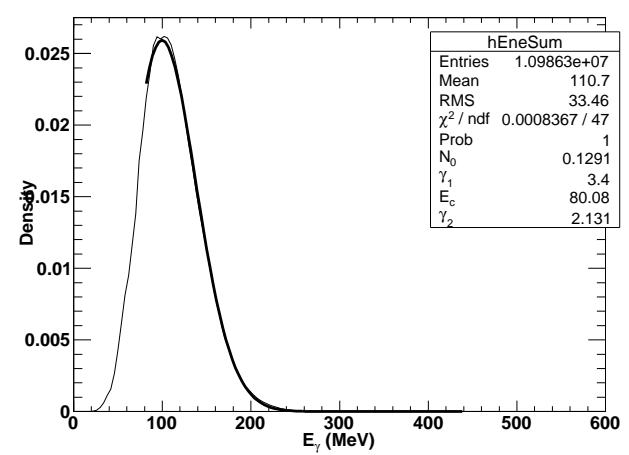

(f) $n=7$

Figure A.4: Histograms of the differential flux distributions determined for source PSR J0630-2834, determined from Monte Carlo, for different $n$. Each histogram has been fit to a PowerLawSuperExpCutoff model, using MINUIT optimizer. 


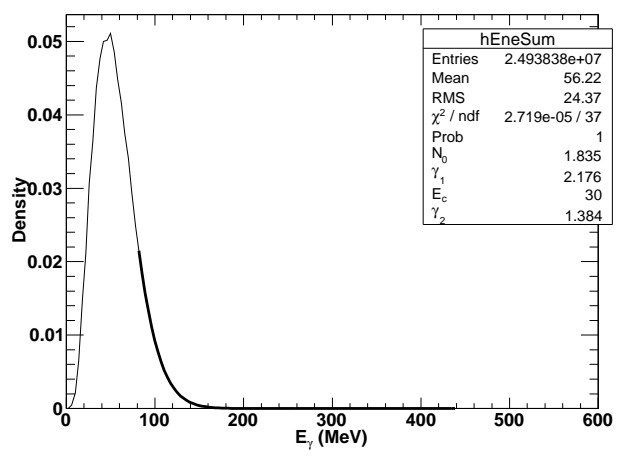

(a) $n=2$

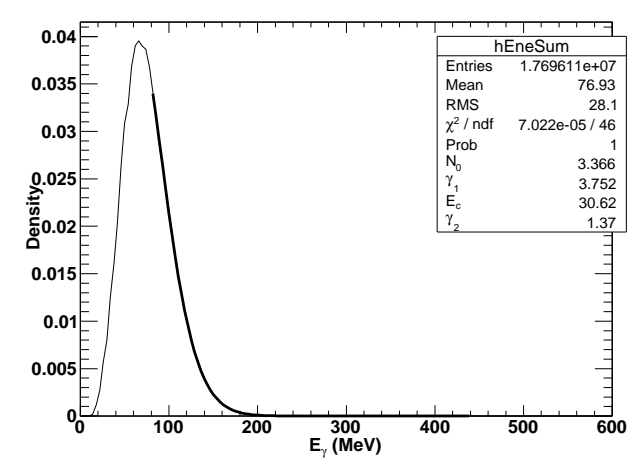

(c) $n=4$

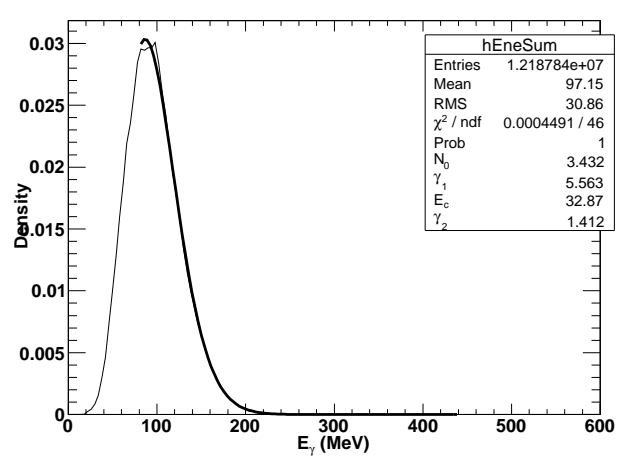

(e) $n=6$

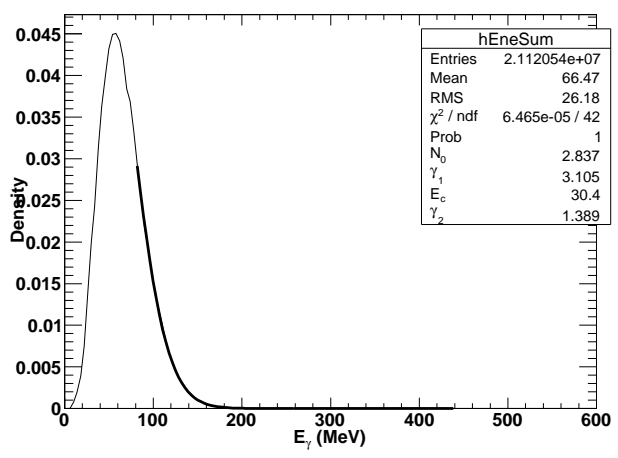

(b) $n=3$

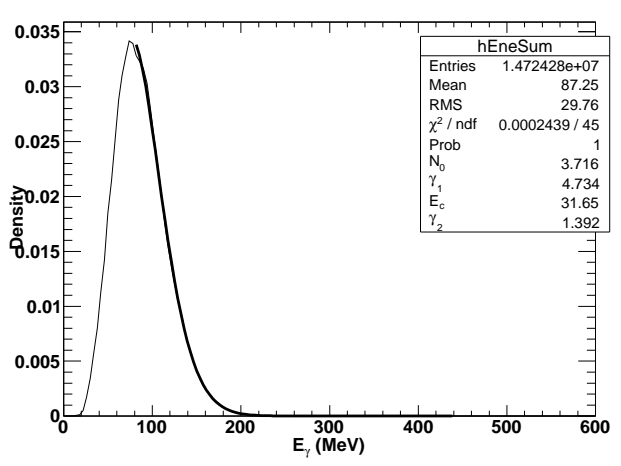

(d) $n=5$

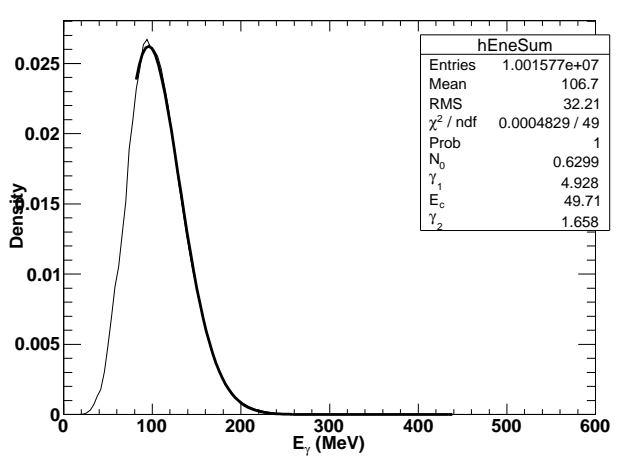

(f) $n=7$

Figure A.5: Histograms of the differential flux distributions, for source PSR J1136+1551, determined from Monte Carlo. Each histogram has been fit to a PowerLawSuperExpCutoff model, using MINUIT optimizer. 


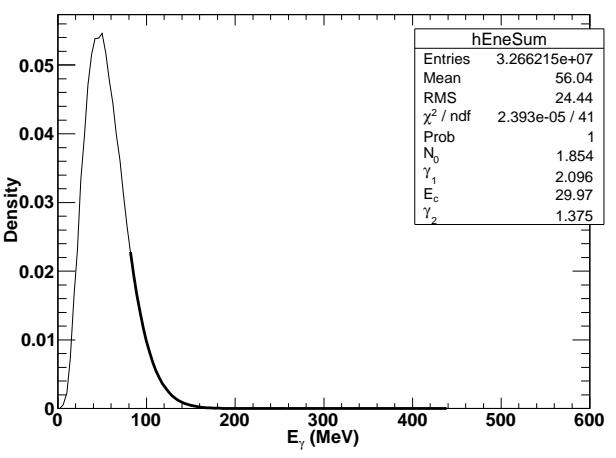

(a) $n=2$

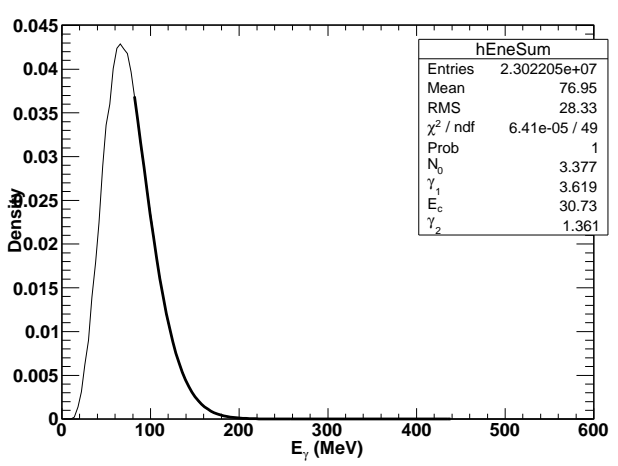

(c) $n=4$

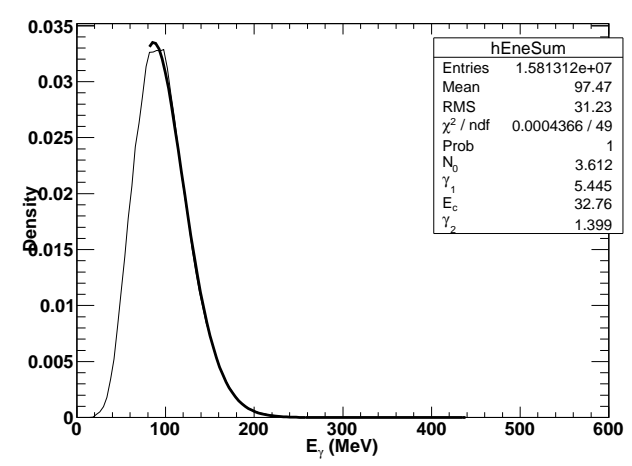

(e) $n=6$

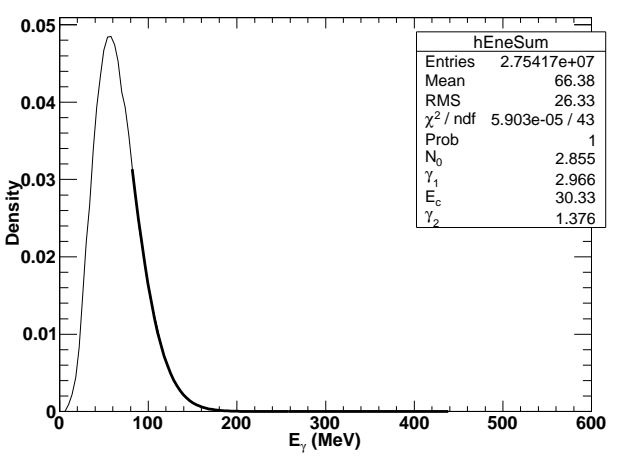

(b) $n=3$

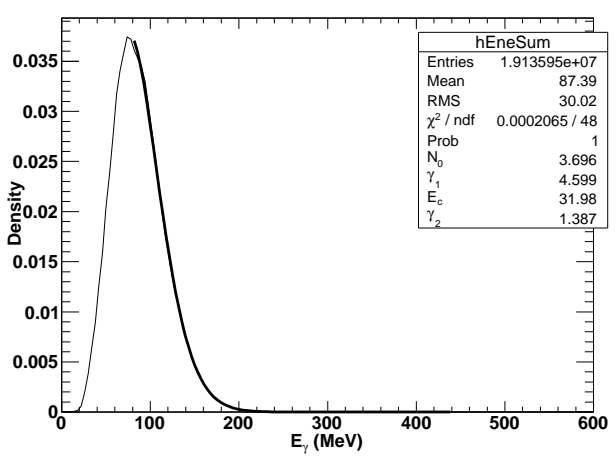

(d) $n=5$

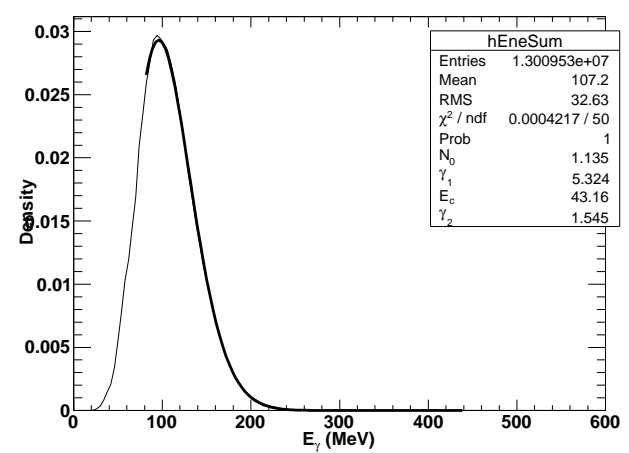

(f) $n=7$

Figure A.6: Histograms of the differential flux distributions, for source PSR J08262637, determined from Monte Carlo, for different $n$. Each histogram has been fit to a PowerLawSuperExpCutoff model, using MINUIT optimizer. 
118APPENDIX A. FITTED DIFFERENTIAL FLUX DISTRIBUTIONS FOR EACH NS SOURCE 


\section{Appendix B}

\section{Sources in the Vicinity of Neutron Stars}

Tables of sources within a $14^{\circ}$ radius region surrounding each neutron star source are presented below, according to values in the 1FGL catalog. 


\begin{tabular}{|l|l|l|l|l|l|l|}
\hline name & RA & Dec & Dist & Index & Integral & TS \\
\hline J1747.4-4035 & 266.860 & -40.589 & 13.886 & -2.141 & $2.57 \mathrm{E}-08$ & 47.60 \\
\hline J1802.5-3939 & 270.636 & -39.662 & 10.888 & -2.254 & $1.96 \mathrm{E}-07$ & 1207.35 \\
\hline J1807.6-4341 & 271.910 & -43.699 & 11.405 & -1.992 & $1.00 \mathrm{E}-08$ & 25.25 \\
\hline J1820.5-3216 & 275.149 & -32.278 & 8.821 & -2.234 & $3.83 \mathrm{E}-08$ & 87.67 \\
\hline J1823.4-3009 & 275.857 & -30.160 & 9.775 & -2.246 & $4.36 \mathrm{E}-08$ & 106.84 \\
\hline J1823.5-3454 & 275.896 & -34.913 & 7.019 & -1.701 & $8.87 \mathrm{E}-09$ & 90.65 \\
\hline J1835.3-3255 & 278.833 & -32.929 & 6.022 & -2.024 & $1.44 \mathrm{E}-08$ & 46.06 \\
\hline J1849.6-4314 & 282.404 & -43.237 & 6.288 & -2.175 & $2.09 \mathrm{E}-08$ & 68.51 \\
\hline J1902.0-5110 & 285.522 & -51.170 & 14.112 & -2.102 & $3.70 \mathrm{E}-08$ & 220.36 \\
\hline J1903.8-3718c & 285.967 & -37.309 & 1.465 & -2.184 & $2.99 \mathrm{E}-08$ & 71.55 \\
\hline J1904.4-2725 & 286.103 & -27.427 & 9.805 & -2.170 & $1.71 \mathrm{E}-08$ & 38.50 \\
\hline J1916.9-3028 & 289.235 & -30.470 & 7.854 & -1.914 & $7.50 \mathrm{E}-09$ & 35.40 \\
\hline J1918.4-4108 & 289.622 & -41.141 & 5.865 & -1.845 & $1.78 \mathrm{E}-08$ & 160.55 \\
\hline J1925.2-2919 & 291.322 & -29.317 & 9.815 & -2.403 & $4.00 \mathrm{E}-08$ & 85.60 \\
\hline J1925.4-3811 & 291.362 & -38.190 & 5.815 & -2.146 & $1.56 \mathrm{E}-08$ & 37.55 \\
\hline J1936.9-4720 & 294.229 & -47.343 & 12.659 & -1.675 & $5.08 \mathrm{E}-09$ & 79.99 \\
\hline J1938.2-3957 & 294.567 & -39.961 & 8.634 & -2.452 & $5.99 \mathrm{E}-08$ & 145.15 \\
\hline J1946.1-3118 & 296.549 & -31.306 & 11.761 & -2.190 & $1.71 \mathrm{E}-08$ & 43.77 \\
\hline J1958.4-3847 & 299.610 & -38.796 & 12.297 & -2.468 & $7.61 \mathrm{E}-08$ & 273.45 \\
\hline J1959.3-4241 & 299.829 & -42.697 & 13.245 & -2.408 & $3.74 \mathrm{E}-08$ & 102.59 \\
\hline
\end{tabular}

Table B.1: Table of 1FGL sources $14^{\circ}$ of RXJ1856-3754 


\begin{tabular}{|l|l|l|l|l|l|l|}
\hline name & RA & Dec. & Dist. & Index & Integral & TS \\
\hline J0022.2-1850 & 5.569 & -18.847 & 11.868 & -1.563 & $3.85 \mathrm{E}-09$ & 101.90 \\
\hline J0141.7-0929 & 25.426 & -9.488 & 9.540 & -2.114 & $1.66 \mathrm{E}-08$ & 130.78 \\
\hline J0033.5-1921 & 8.391 & -19.365 & 9.670 & -1.887 & $2.01 \mathrm{E}-08$ & 322.21 \\
\hline J0034.3-0534 & 8.591 & -5.577 & 12.083 & -2.187 & $3.56 \mathrm{E}-08$ & 245.58 \\
\hline J0038.4-2504 & 9.609 & -25.075 & 12.793 & -2.447 & $2.79 \mathrm{E}-08$ & 99.12 \\
\hline J0047.3-2512 & 11.827 & -25.208 & 11.908 & -2.149 & $1.15 \mathrm{E}-08$ & 48.68 \\
\hline J0050.0-0446 & 12.512 & -4.782 & 10.555 & -2.341 & $1.67 \mathrm{E}-08$ & 41.26 \\
\hline J0050.6-0928 & 12.665 & -9.483 & 6.478 & -2.196 & $7.43 \mathrm{E}-08$ & 769.76 \\
\hline J0054.9-2455 & 13.734 & -24.929 & 11.024 & -1.954 & $5.53 \mathrm{E}-09$ & 37.02 \\
\hline J0051.1-0649 & 12.783 & -6.818 & 8.613 & -2.356 & $4.34 \mathrm{E}-08$ & 178.95 \\
\hline J0104.4-2406 & 16.100 & -24.107 & 9.795 & -2.277 & $1.12 \mathrm{E}-08$ & 29.64 \\
\hline J0115.5-1132 & 18.875 & -11.549 & 3.327 & -2.485 & $1.79 \mathrm{E}-08$ & 34.81 \\
\hline J0118.7-2137 & 19.683 & -21.628 & 7.699 & -2.399 & $8.31 \mathrm{E}-08$ & 679.95 \\
\hline J0120.5-2700 & 20.131 & -27.016 & 12.990 & -1.993 & $3.56 \mathrm{E}-08$ & 518.38 \\
\hline J0124.6-0616 & 21.154 & -6.283 & 9.027 & -2.204 & $1.22 \mathrm{E}-08$ & 40.70 \\
\hline J0132.6-1655 & 23.163 & -16.927 & 6.439 & -2.559 & $8.87 \mathrm{E}-08$ & 553.99 \\
\hline J0136. 3-2220 & 24.081 & -22.340 & 10.414 & -1.600 & $1.68 \mathrm{E}-09$ & 30.69 \\
\hline J0137.5-2428 & 24.393 & -24.482 & 12.272 & -2.449 & $2.87 \mathrm{E}-08$ & 109.20 \\
\hline J0141.7-0929 & 25.426 & -9.488 & 9.540 & -2.114 & $1.66 \mathrm{E}-08$ & 130.78 \\
\hline J0205.0-1702 & 31.261 & -17.039 & 13.953 & -2.534 & $4.83 \mathrm{E}-08$ & 202.96 \\
\hline
\end{tabular}

Table B.2: Table of 1FGL sources within $14^{\circ}$ of PSR J0108-1431.

\begin{tabular}{|l|l|l|l|l|l|l|}
\hline name & RA & Dec & Dist & Index & Integral & TS \\
\hline J0905.5+1356 & 136.392 & 13.948 & 13.136 & -1.949 & $9.52 \mathrm{E}-09$ & 94.62 \\
\hline J0909.0+0126 & 137.273 & 1.444 & 12.745 & -2.738 & $1.33 \mathrm{E}-07$ & 681.90 \\
\hline J0926.9+1452 & 141.740 & 14.879 & 9.460 & -2.731 & $2.43 \mathrm{E}-08$ & 30.84 \\
\hline J0946.6+1012 & 146.672 & 10.207 & 2.784 & -2.499 & $2.63 \mathrm{E}-08$ & 61.10 \\
\hline J0949.0+0021 & 147.250 & 0.364 & 7.633 & -2.662 & $1.35 \mathrm{E}-07$ & 638.12 \\
\hline J0949.8+1757 & 147.452 & 17.955 & 10.061 & -2.441 & $1.49 \mathrm{E}-08$ & 34.91 \\
\hline J1007.9+0619 & 151.989 & 6.327 & 4.004 & -2.333 & $2.72 \mathrm{E}-08$ & 89.75 \\
\hline J1011.0-0156 & 152.757 & -1.933 & 10.821 & -2.228 & $1.22 \mathrm{E}-08$ & 40.17 \\
\hline J1012.2+0634 & 153.061 & 6.572 & 4.924 & -2.296 & $1.51 \mathrm{E}-08$ & 47.22 \\
\hline J1016.1+0514 & 154.032 & 5.235 & 6.308 & -2.277 & $1.23 \mathrm{E}-07$ & 1231.15 \\
\hline J1022.8-0115 & 155.723 & -1.250 & 11.797 & -1.934 & $6.86 \mathrm{E}-09$ & 49.94 \\
\hline J1040.5+0616 & 160.127 & 6.278 & 11.862 & -2.562 & $4.98 \mathrm{E}-08$ & 170.00 \\
\hline
\end{tabular}

Table B.3: Table of 1FGL sources within $14^{\circ}$ of PSR J0953+0755. 


\begin{tabular}{|l|l|l|l|l|l|l|}
\hline name & RA & Dec & Dist & Index & Integral & TS \\
\hline J0536.2-3348 & 84.061 & -33.803 & 12.774 & -2.425 & $6.66 \mathrm{E}-08$ & 366.83 \\
\hline J0539.1-2847 & 84.790 & -28.792 & 11.327 & -2.706 & $2.96 \mathrm{E}-08$ & 45.48 \\
\hline J0557.6-3831 & 89.406 & -38.526 & 12.103 & -2.317 & $1.75 \mathrm{E}-08$ & 43.68 \\
\hline J0600.5-2006 & 90.150 & -20.113 & 10.905 & -2.410 & $2.40 \mathrm{E}-08$ & 48.70 \\
\hline J0603.0-4012 & 90.765 & -40.213 & 12.957 & -2.253 & $1.68 \mathrm{E}-08$ & 41.55 \\
\hline J0610.7-2059 & 92.683 & -20.998 & 8.844 & -2.377 & $2.91 \mathrm{E}-08$ & 78.34 \\
\hline J0614.1-3328 & 93.546 & -33.479 & 6.058 & -1.930 & $1.25 \mathrm{E}-07$ & 2986.09 \\
\hline J0617.7-1718 & 94.427 & -17.310 & 11.665 & -1.990 & $1.22 \mathrm{E}-08$ & 79.43 \\
\hline J0622.3-2604 & 95.585 & -26.073 & 3.135 & -2.005 & $1.13 \mathrm{E}-08$ & 61.93 \\
\hline J0627.3-3530 & 96.831 & -35.504 & 6.965 & -1.861 & $6.74 \mathrm{E}-09$ & 50.41 \\
\hline J0629.6-2000 & 97.406 & -20.012 & 8.571 & -2.205 & $1.99 \mathrm{E}-08$ & 61.63 \\
\hline J0630.9-2406 & 97.747 & -24.107 & 4.472 & -1.871 & $2.28 \mathrm{E}-08$ & 313.60 \\
\hline J0648.7-1740 & 102.188 & -17.670 & 11.658 & -2.469 & $7.59 \mathrm{E}-08$ & 130.44 \\
\hline J0650.6-1635 & 102.672 & -16.592 & 12.829 & -2.457 & $4.25 \mathrm{E}-08$ & 44.46 \\
\hline J0651.1-3022 & 102.780 & -30.371 & 4.766 & -2.310 & $1.56 \mathrm{E}-08$ & 28.17 \\
\hline J0702.2-1954 & 105.560 & -19.901 & 11.244 & -1.917 & $8.93 \mathrm{E}-09$ & 26.21 \\
\hline J0724.1-3522 & 111.039 & -35.369 & 13.173 & -2.445 & $3.11 \mathrm{E}-08$ & 46.99 \\
\hline J0724.7-2223c & 111.182 & -22.383 & 13.638 & -2.504 & $4.93 \mathrm{E}-08$ & 44.57 \\
\hline
\end{tabular}

Table B.4: Table of 1FGL sources within $14^{\circ}$ of PSR J0630-2834. 


\begin{tabular}{|l|l|l|l|l|l|l|}
\hline name & RA & Dec & Dist & Index & Integral & TS \\
\hline J1048.7+2335 & 162.189 & 23.596 & 13.549 & -2.073 & $1.10 \mathrm{E}-08$ & 51.36 \\
\hline J1054.5+2212 & 163.627 & 22.210 & 11.692 & -2.284 & $3.48 \mathrm{E}-08$ & 206.12 \\
\hline J1101.3+1009 & 165.327 & 10.157 & 10.197 & -1.575 & $9.87 \mathrm{E}-10$ & 25.32 \\
\hline J1104.4+0734 & 166.112 & 7.581 & 11.320 & -2.216 & $1.85 \mathrm{E}-08$ & 88.50 \\
\hline J1107.8+1502 & 166.970 & 15.050 & 6.836 & -1.949 & $7.43 \mathrm{E}-09$ & 55.30 \\
\hline J1112.3+0458 & 168.094 & 4.968 & 12.338 & -2.299 & $1.12 \mathrm{E}-08$ & 27.59 \\
\hline J1117.1+2013 & 169.299 & 20.222 & 6.260 & -1.706 & $1.14 \mathrm{E}-08$ & 270.89 \\
\hline J1120.4+0710 & 170.122 & 7.172 & 9.478 & -2.028 & $8.51 \mathrm{E}-09$ & 55.26 \\
\hline J1123.9+2339 & 170.997 & 23.663 & 8.311 & -2.408 & $1.73 \mathrm{E}-08$ & 47.18 \\
\hline J1136.9+2551 & 174.236 & 25.860 & 10.011 & -1.528 & $1.48 \mathrm{E}-09$ & 39.03 \\
\hline J1141.8+1549 & 175.471 & 15.821 & 1.402 & -2.058 & $6.14 \mathrm{E}-09$ & 27.62 \\
\hline J1150.2+2419 & 177.566 & 24.321 & 9.102 & -2.254 & $2.09 \mathrm{E}-08$ & 102.38 \\
\hline J1204.4+1139 & 181.124 & 11.665 & 8.074 & -2.149 & $1.12 \mathrm{E}-08$ & 49.39 \\
\hline J1209.7+1806 & 182.445 & 18.100 & 8.370 & -2.286 & $1.60 \mathrm{E}-08$ & 55.96 \\
\hline J1224.7+2121 & 186.199 & 21.363 & 12.789 & -2.547 & $8.10 \mathrm{E}-08$ & 449.57 \\
\hline J1230.8+1223 & 187.721 & 12.394 & 13.731 & -2.326 & $2.82 \mathrm{E}-08$ & 129.43 \\
\hline
\end{tabular}

Table B.5: Table of 1FGL sources within $14^{\circ}$ of PSR J1136+1551. 


\begin{tabular}{|l|l|l|l|l|l|l|}
\hline name & RA & Dec & Dist & Index & Integral & TS \\
\hline J0730.0+3305 & 112.516 & 33.090 & 13.890 & -2.090 & $7.89 \mathrm{E}-09$ & 41.22 \\
\hline J0746.6+2548 & 116.660 & 25.804 & 9.054 & -2.742 & $4.91 \mathrm{E}-08$ & 113.40 \\
\hline J0751.1+1807 & 117.788 & 18.127 & 11.836 & -2.155 & $3.53 \mathrm{E}-08$ & 248.62 \\
\hline J0809.4+3455 & 122.354 & 34.933 & 9.113 & -1.775 & $3.27 \mathrm{E}-09$ & 32.07 \\
\hline J0830.5+2407 & 127.635 & 24.122 & 2.636 & -2.788 & $8.38 \mathrm{E}-08$ & 274.31 \\
\hline J0840.8+1310 & 130.210 & 13.177 & 13.840 & -2.437 & $2.85 \mathrm{E}-08$ & 78.95 \\
\hline J0847.4+1517 & 131.858 & 15.284 & 12.311 & -2.475 & $1.31 \mathrm{E}-08$ & 30.44 \\
\hline J0850.2+3457 & 132.567 & 34.961 & 9.731 & -2.035 & $5.80 \mathrm{E}-09$ & 30.55 \\
\hline J0854.8+2006 & 133.710 & 20.109 & 9.144 & -2.383 & $7.05 \mathrm{E}-08$ & 376.08 \\
\hline J0856.6+2103 & 134.158 & 21.062 & 8.787 & -2.218 & $1.22 \mathrm{E}-08$ & 34.72 \\
\hline J0900.5+3410 & 135.136 & 34.169 & 10.466 & -2.238 & $9.96 \mathrm{E}-09$ & 25.66 \\
\hline J0902.4+2050 & 135.600 & 20.848 & 9.971 & -2.092 & $1.57 \mathrm{E}-08$ & 93.44 \\
\hline J0909.2+2310 & 137.300 & 23.176 & 10.198 & -1.459 & $2.40 \mathrm{E}-09$ & 66.69 \\
\hline J0910.7+3332 & 137.680 & 33.535 & 11.728 & -2.316 & $2.01 \mathrm{E}-08$ & 73.05 \\
\hline J0911.0+2247 & 137.764 & 22.785 & 10.742 & -2.213 & $3.40 \mathrm{E}-08$ & 137.74 \\
\hline J0912.6+2756 & 138.154 & 27.936 & 10.248 & -1.332 & $6.31 \mathrm{E}-10$ & 25.37 \\
\hline J0915.7+2931 & 138.941 & 29.532 & 11.167 & -1.943 & $1.70 \mathrm{E}-08$ & 202.04 \\
\hline J0922.0+2337 & 140.513 & 23.619 & 12.843 & -1.662 & $2.08 \mathrm{E}-09$ & 30.91 \\
\hline J0924.2+2812 & 141.057 & 28.202 & 12.823 & -2.491 & $3.37 \mathrm{E}-08$ & 111.84 \\
\hline
\end{tabular}

Table B.6: Table of 1FGL sources within $14^{\circ}$ of PSR J0826-2637. 


\section{Appendix C}

\section{Fitting Catalog Defined ROIs}

\section{C.0.1 Introduction}

The first year Fermi gamma ray LAT catalog was compiled from characterization of the spectra of detected sources over 11 month period beginning in August 2008. The methodology and results of the catalog are applicable to the dissertation for the several reasons. One reason is that many background sources listed in the catalog are located within the ROIs of faint neutron stars whose spectra are being modeled. Another is that the application of similar methods as used by the catalog authors will be useful for spectral characterization of the faint gamma-ray neutron stars.

Here a brief overview of the construction of the catalog from the 1 year catalog paper is presented [78]. Many steps involved in the compiling of the catalog include detection, localization, global fit, and flux determination.

An initial pass was made over the entire sky partitioned in 24 planar projections in a detection step, searching for sources above approximately $4 \sigma$ significance. Wavelet based detection methods, namely $m r_{-}$filter and $P G$ Wave, in addition to a maximum likelihood method pointfind and a minimum spanning tree algorithm, were employed as detection methods. Localization fits for both the position and extension. For spectral characterization, the sky was broken into 445 overlapping circular ROIs between $9^{\circ}$ and $15^{\circ}$ in radius. A global power law fit was used to find spectral index and flux estimate over the range of $\gamma$-ray energies $100 \mathrm{MeV}$ to $100 \mathrm{GeV}$. Integral photon flux 
values (photons $\mathrm{cm}^{-2} \mathrm{~S}^{-1}$ were determined in 5 predefined energy bands $100 \mathrm{MeV}$ $300 \mathrm{MeV}, 300 \mathrm{MeV}-1 \mathrm{GeV}, 1 \mathrm{GeV}-3 \mathrm{GeV}, 3 \mathrm{GeV}-10 \mathrm{GeV}, 10 \mathrm{GeV}-100 \mathrm{GeV}$. In some of the bands, upper limits were provided when the TS was low.

\section{C.0.2 Fitting ROIs}

According to the catalog prescription for fitting sources within an ROI, only fit for sources within the central part of each ROI, having a radius of ROI radius minus $7^{\circ}$. We take as an example of fitting a particular ROI, ROI \# 32. This ROI was chosen as an example of fitting a mid-Galactic latitude ROI, centered at $(\ell, b)=$ $\left(21.589^{\circ}, 44.010^{\circ}\right)$, or $(\mathrm{RA}, \mathrm{Dec})=\left(238.780^{\circ}, 11.006^{\circ}\right)$. The list of sources modeled by the catalog, their positions, their fluxes in each energy band, which ROI they belong to, their TS values, the ROI definitions, and other useful information are publicly available from the FITS file gll_psc11month_v2.fit [79]. The galactic diffuse was modeled as the collaboration-developed MapCubeFunction gll_iem_v02.fit, multiplied by a power law function with free index and normalization. The isotropic component, the extra-galactic diffuse, was taken as function determined in log-spaced energy bins, in a file developed by the collaboration called isotropic_iem_v02.txt. Version $v 9 r 15 p 6$ of the ScienceTools was used for making subselections of the data, making GTIs, making livetime cubes, developing exposure maps, and gtlike was used in fitting. Unbinned mode of maximum likelihood was applied in keeping with the catalog. In addition to sources within the central part of the ROI having free parameters, sources within $2^{\circ}$ of any source inside the central part of the ROI and sources having a relatively high flux (i.e., $5 \%$ or more of the total counts within the ROI) were allowed to have integral flux as a free parameter. Two separate fits were attempted: (1) allowing the fluxes free for sources within the central part of the ROI, while fixing the index to the catalog value; (2) allowing the fluxes and the spectral indices to be free in the unbinned likelihood fit.

\section{C.0.3 Fitting ROI Results}


Figure C.1: Counts map in ROI 32 for photon energies between $300 \mathrm{MeV}$ and $1 \mathrm{GeV}$.

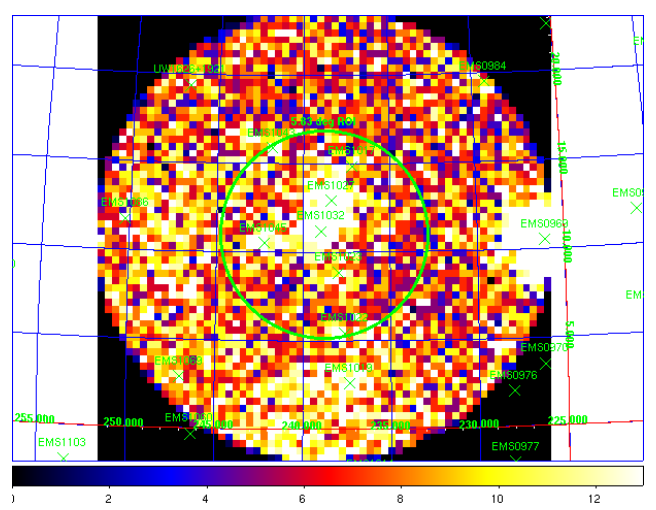

\begin{tabular}{|c|c|c|c|c|c|c|c|}
\hline 1FGL Name & $\begin{array}{l}\text { RA } \\
\left({ }^{\circ}\right)\end{array}$ & $\begin{array}{l}\text { Dec. } \\
\left({ }^{\circ}\right)\end{array}$ & $\begin{array}{l}\text { Dist. } \\
\left({ }^{\circ}\right)\end{array}$ & Index & $\begin{array}{l}\text { Integral } \\
\left(\mathrm{cm}^{-2} \mathrm{~s}^{-1}\right)\end{array}$ & $\begin{array}{l}\text { Catalog } \\
\text { Index }\end{array}$ & $\begin{array}{l}\text { Catalog } \\
\text { Integral } \\
\left(\mathrm{cm}^{-2} \mathrm{~s}^{-1}\right)\end{array}$ \\
\hline $\mathrm{J} 1555.7+1111$ & $\overline{238.94}$ & 11.19 & 0.24 & $\begin{array}{l}-1.675 \\
\pm 0.036\end{array}$ & $\begin{array}{l}6.42 \mathrm{E}-08 \\
\pm 5.06 \mathrm{E}-09\end{array}$ & $\begin{array}{l}-1.664 \\
\pm 0.031\end{array}$ & $\begin{array}{l}6.60 \mathrm{E}-08 \\
\pm 4.60 \mathrm{E}-09\end{array}$ \\
\hline J1553.4+1255 & 238.36 & 12.93 & 1.96 & $\begin{array}{l}-2.283 \\
\pm 0.065\end{array}$ & $\begin{array}{l}1.23 \mathrm{E}-07 \\
\pm 1.54 \mathrm{E}-8\end{array}$ & $\begin{array}{l}-2.299 \\
\pm 0.042\end{array}$ & $\begin{array}{l}1.27 \mathrm{E}-07 \\
\pm 8.76 \mathrm{E}-09\end{array}$ \\
\hline $\mathrm{J} 1551.7+0851$ & 237.95 & 8.85 & 2.30 & $\begin{array}{l}-2.192 \\
\pm 0.446\end{array}$ & $\begin{array}{l}9.97 \mathrm{E}-09 \\
\pm 1.01 \mathrm{E}-08\end{array}$ & $\begin{array}{l}-2.213 \\
\pm 0.19\end{array}$ & $\begin{array}{l}1.07 \mathrm{E}-08 \\
\pm 4.54 \mathrm{E}-09\end{array}$ \\
\hline J1609.0+1031 & 242.25 & 10.53 & 3.45 & $\begin{array}{l}-2.720 \\
\pm 0.130\end{array}$ & $\begin{array}{l}6.20 \mathrm{E}-08 \\
\pm 1.08 \mathrm{E}-08\end{array}$ & $\begin{array}{l}-2.717 \\
\pm 0.104\end{array}$ & $\begin{array}{l}6.19 \mathrm{E}-08 \\
\pm 8.68 \mathrm{E}-09\end{array}$ \\
\hline J1548.6+1451 & 237.15 & 14.85 & 4.17 & $\begin{array}{l}-2.191 \\
\pm 0.789\end{array}$ & $\begin{array}{l}1.68 \mathrm{E}-08 \\
\pm 2.72 \mathrm{E}-08\end{array}$ & $\begin{array}{l}-2.182 \\
\pm 0.149\end{array}$ & $\begin{array}{l}1.62 \mathrm{E}-08 \\
\pm 5.07 \mathrm{E}-09\end{array}$ \\
\hline J1607.1+1552 & 241.79 & 15.87 & 5.68 & $\begin{array}{l}-2.247 \\
\pm 0.110\end{array}$ & $\begin{array}{l}3.77 \mathrm{E}-08 \\
\pm 7.52 \mathrm{E}-09\end{array}$ & $\begin{array}{l}-2.248 \\
\pm 0.085\end{array}$ & $\begin{array}{l}3.86 \mathrm{E}-08 \\
\pm 5.93 \mathrm{E}-09\end{array}$ \\
\hline
\end{tabular}

Table C.1: Table of integral fluxes and spectral indices determined from my fit as compared with the catalog values. 


\section{Appendix D}

\section{Studies with Monte Carlo of Low Energy Gamma Rays}

An investigation on using the parametric energy variable EvtEnergyCorr instead of the default analysis energy variable CTBBestEnergy was performed. In addition, a comparison of P6_V3_DIFFUSE and P7_V2_DIFFUSE was performed, as part of validation studies for the newer IRF framework, Pass 7.

\section{D.1 Introduction}

The Monte Carlo simulation of the LAT is based on the Geant4 Monte Carlo toolkit, which is an object-oriented simulator of the passage of particles through matter and detectors [80]. Geant4 models nearly all of the particle interaction with matter encountered in high-energy physics. Processes of interest to Fermi-LAT such as pairproduction and Compton scattering of photons, bremsstrahlung for electrons and positrons, multiple scattering and $\delta$-ray production for all charged particles, lowenergy interaction with atoms, and hadronic interactions are readily handled by Geant4. A custom implementation of the Geant4 simulation for the LAT, known as Gleam, was developed by the collaboration 81]. The simulation of the instrument and response is comprised of 3 main steps. First, particle simulation of particle interaction to model interaction of incident $\gamma$-rays and background particles on the LAT. 
Second, the instrument response (digitization) is computed parametrically from energy deposition and localization in the various segments of the ACD, the tracker, and the calorimeter. Third, from the digitized instrument responses, a set of instrument triggers is generated and a copy of the Trigger and On-Board Flight Software Filter is applied to the simulated data stream.

Since the science analysis depends crucially on the low energy measurement, a study of variables measuring energy below $400 \mathrm{MeV}$ was done on Monte Carlo samples of photons incident on the LAT. A set of cuts, i.e. selection on variables, was developed to determine a nearly vertically incident set of photons. Another aim was to "validate" one set of Instrument Response Functions (IRFs) for the Fermi-LAT under development in 2009-2010, Pass 7 Version 2 P7_V2, as compared with a baseline of Pass 6 Version 3, henceforth P6_V3.

A classification tree algorithm was developed by Fermi-LAT colloborators, developed in Insightful Miner, was used in the selection of photon into classes, termed transient, source, and diffuse, associated with CTBClassLevel variable being 1, 2, or 3, respectively. The loosest photon event class selection was chosen: transient, source, and diffuse class photons were included in the analysis, as selected by the selection CTBClassLevel $>0.99$. The criteria and motivation for additional cuts is described as follows. The next two cuts were required for the above event classes. a cut was made on the On Board Filter variable status, corresponding to selection ObfGamState $==0$. The CTBCORE variable, whcih corresponds to the classification-tree assigned probability that direction solutions are good, was required to be greater than 0 . The number of tracks based on conversions in the tracker was selected to be at least one, TkrNumTracks $>0$. The true value of the cosine of the angle from normal incidence, McZDir, was required to have value between -1 and -0.8, with -1 corresponding to complete normal incidence in the negative $Z$-direction. In addition, conversion in the first 8 layers of the tracker was required for selection of events well within the front section of the tracker, as Tkr1ZDir $\geq 11$. Additional cuts to remove charged particle background by data from the ACD were included: AcdTileCount $==0$ as well as AcdRibbonCount $==0$. In addition, a minimal amount of energy deposition was required, to ensure the track is going all the way down into the tracker: CalEnergyRaw 


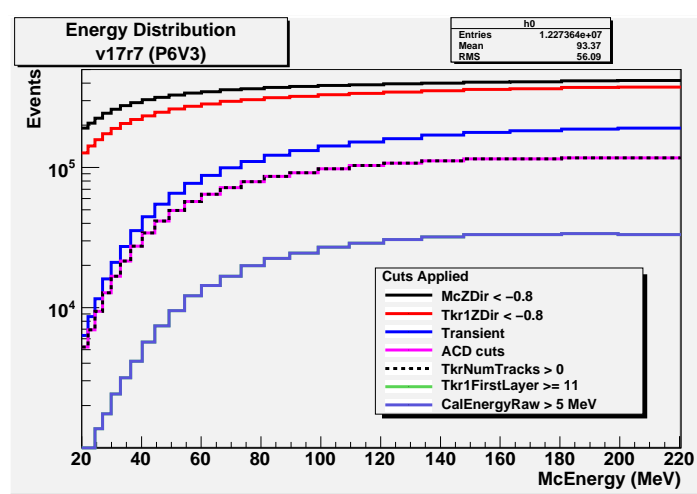

(a) P6V3

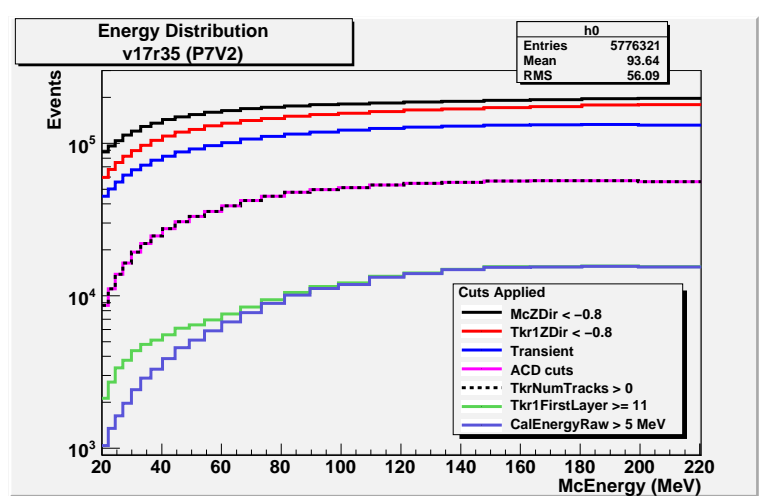

(b) P7V2

Figure D.1: Efficiencies after each successive cut is applied.

$>5 \mathrm{MeV}$.

The Monte Carlo event samples were generated with a $1 / E$ power law spectrum, distributed isotropically over the top of the LAT. The different Monte Carlo samples used in our correspond to 2 different instrument response functions. The samples were all gamma event samples, tagged within the Fermi-LAT collaboration as allGamma-GR-v17r7-lowE-OVRLY and AG-GR-v17r35p1. The efficiency of the various cuts, applied successively, are shown in Figure D.1.

\section{D.2 Comparison of Energy Variable with True En- ergy}

The simulated incident enegy, the Monte Carlo energy or McEnergy, was used as a point of reference for comparing simulated measured energies in the Fermi-LAT instrument. By studying the ratio of measured energy to simulated energy, and plotting, one may characterize the deviations. Also, the residuals should be useful for quantifying the discrepancies. The ratio EvtEnergyCorr/McEnergy, for values between 0.5 to 1.5, is plotted in Figure D.2n different Monte Carlo energy ranges, between 66 and $200 \mathrm{MeV}$. Each histogram was fitted to a double Gaussian, for P6V3 
(left) and P7V2 IRFs (right), The bottom panel on each plot depicts the residuals for each plot. The mean and the effective rms from each fit were used to determine the energy resolution.

\section{D.3 Energy Resolution Studies}

The ratio EvtEnergyCorr/McEnergy may be fitted as a double Gaussian, so that a trend of the mean of the ratio $\langle E\rangle$ and $\sigma(E) /\langle E\rangle$ may be calculated.

Energy resolution for the two different IRFs P6_V3 and P7_V2, and the two different energy variables EvtEnergyCorr and CTBBestEnergy, are shown in Figure D.3. Energy resolution for EvtEnergyCorr generally seems to be better below $100 \mathrm{MeV}$ than that of CTBBestEnergy belolw $100 \mathrm{MeV}$, in either P6_V3 or P7_V2 IRFs. Above $50 \mathrm{MeV}$, the energy resolution can be fit to the form $\left(a^{2} / E+b^{2}+c^{2} / E^{2}\right)^{1 / 2}$, as suggested in the Review of Particle Physics by the Particle Data Group [16] The $a$ term is statistical, owing to shower fluctuations and sampling fluctuations; the $b$ term is due to systematics, such as detector non-uniformity and calibration uncertainty; and the $c$ term is due to electronic noise.

\section{D.4 Calculation of Mean Energy and Energy Res- olution Using Toy MC}

Using a toy Monte Carlo procedure, coordinates and errors for $\langle E\rangle$ and $\sigma(E) / E$ were obtained. The ratio of energy/McEnergy was fit to a double gaussian: each double gaussian has 6 parameters: $p_{0}, p_{1}, . ., p_{5}$ and assoc. errors $e_{0}, e_{1}, \ldots, e_{5}$. The double gaussian function is represented as $f\left(p_{0}, p_{1}, . ., p_{5} ; E\right)$. In this procedure, it is assumed that each parameter is distributed independently according to a gaussian distribution with mean $p_{i}$ and sigma $e_{i}$. The mean $\langle E\rangle$ and $\sigma(E)$ were obtained from the integral over the distribution $f\left(p_{0}, p_{1}, \ldots, p_{5} ; E\right)$ for each Monte Carlo trial.

\footnotetext{
${ }^{1}$ This resolution function can be written as $a / \sqrt{E} \oplus b \oplus c / E$, where $\oplus$ represents addition in quadrature.
} 

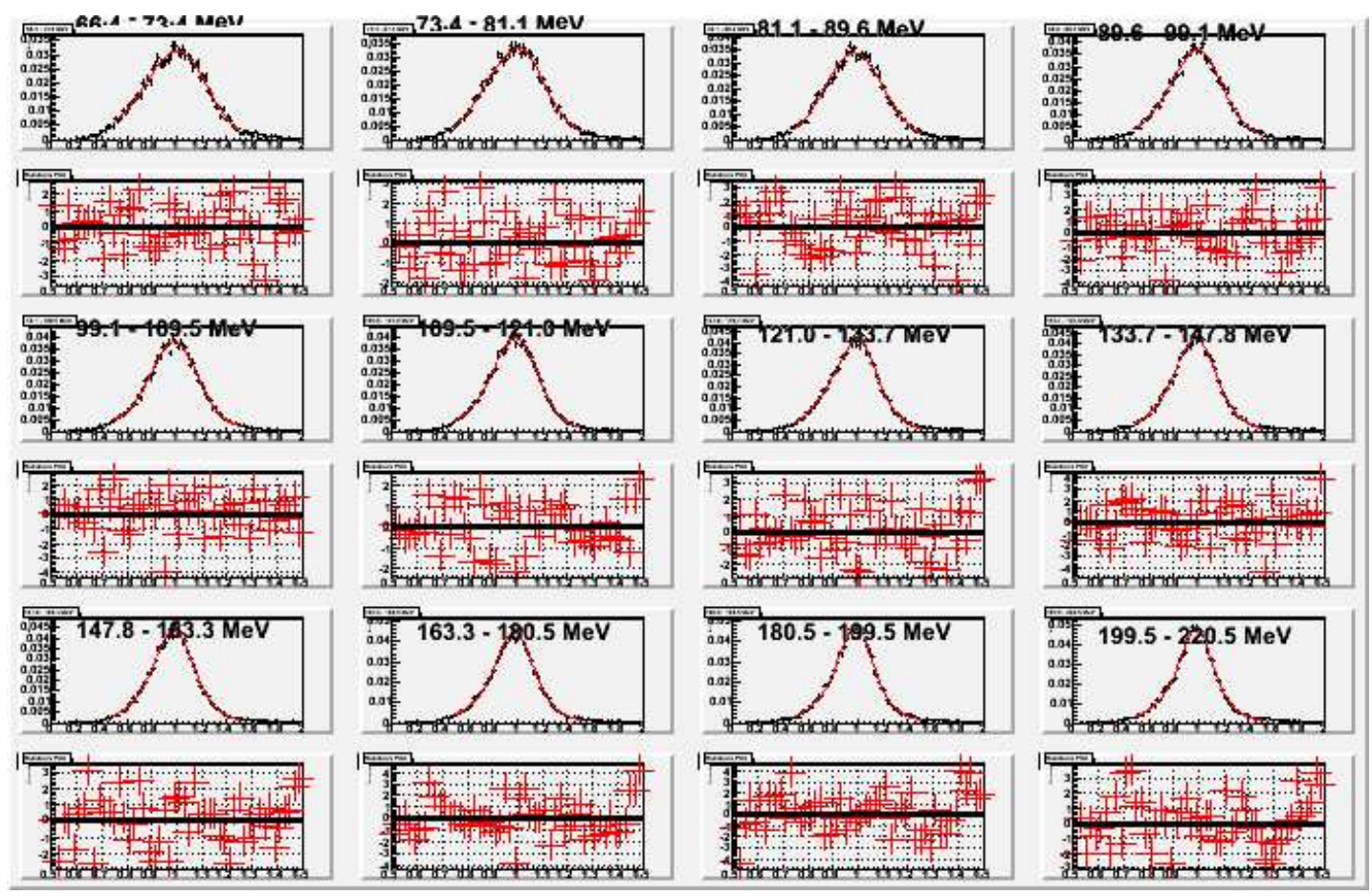

(a) P6_V3
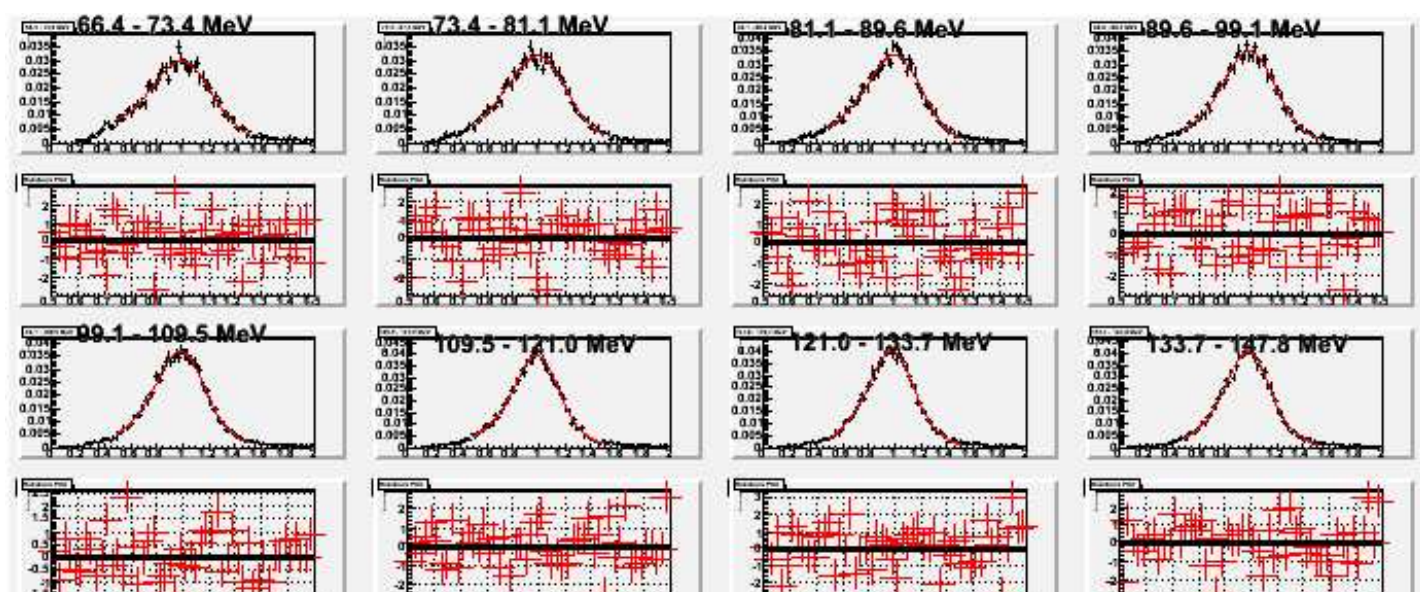

is
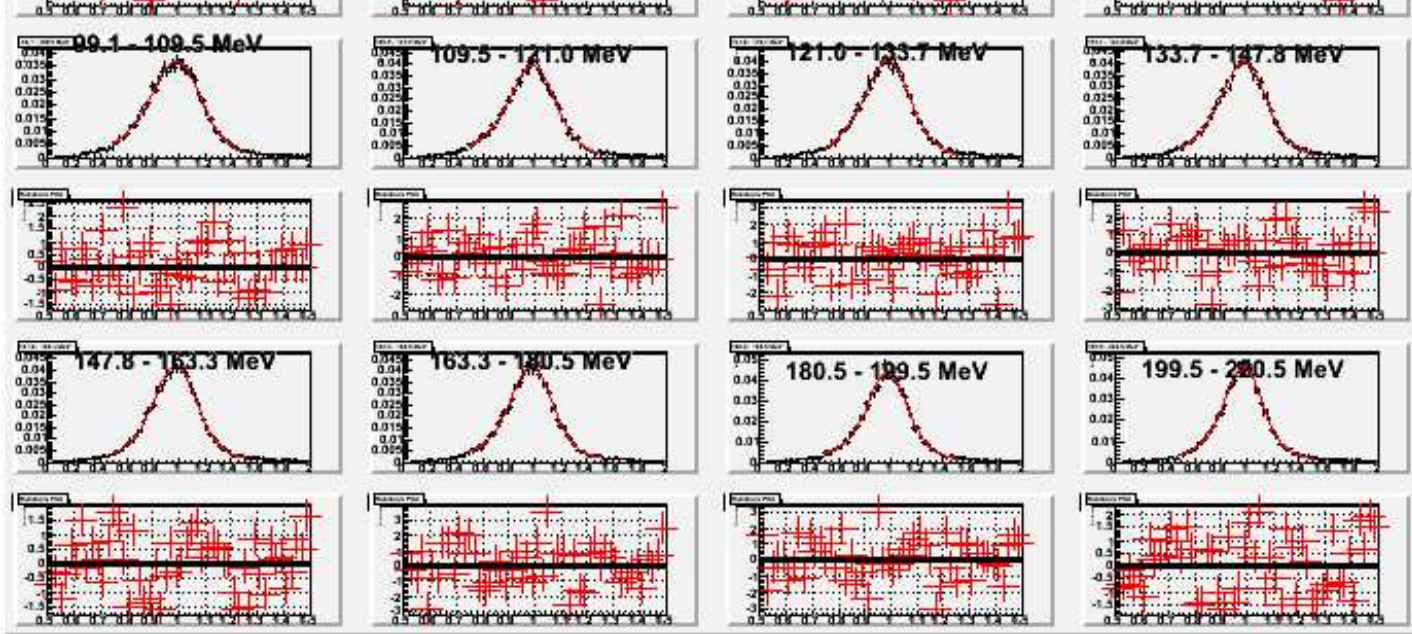

(b) P7_V2

Figure D.2: Plots of the ratio of EvtEnergyCorr/McEnergy for P6V3 and P7V2 IRFs, and residuals to fit. The ratio is plotted in the range 0.5 to 1.5 . 


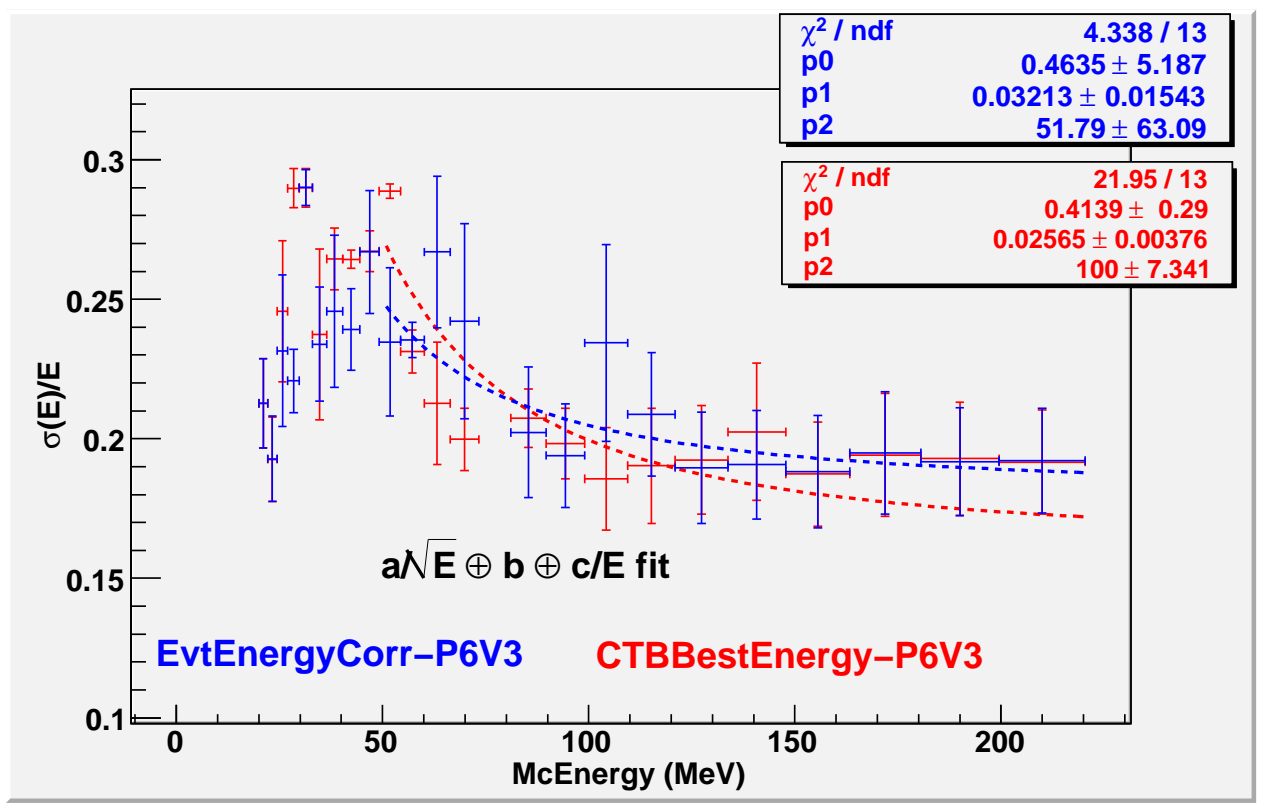

(a) P6_V3

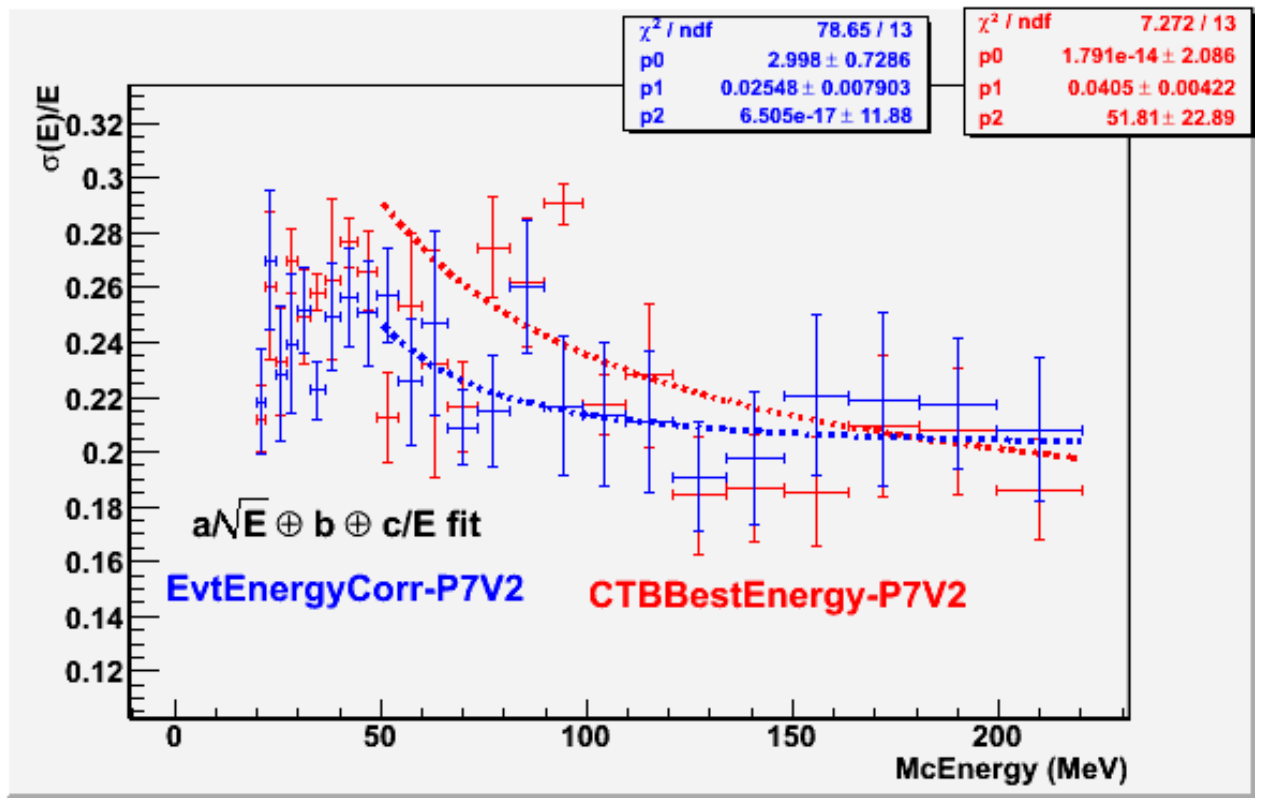

(b) P7_V2

Figure D.3: Energy resolution for two different energy variables EvtEnergyCorr and CTBBestEnergy, for the two different IRFs P6_V3 and P7_V2. 


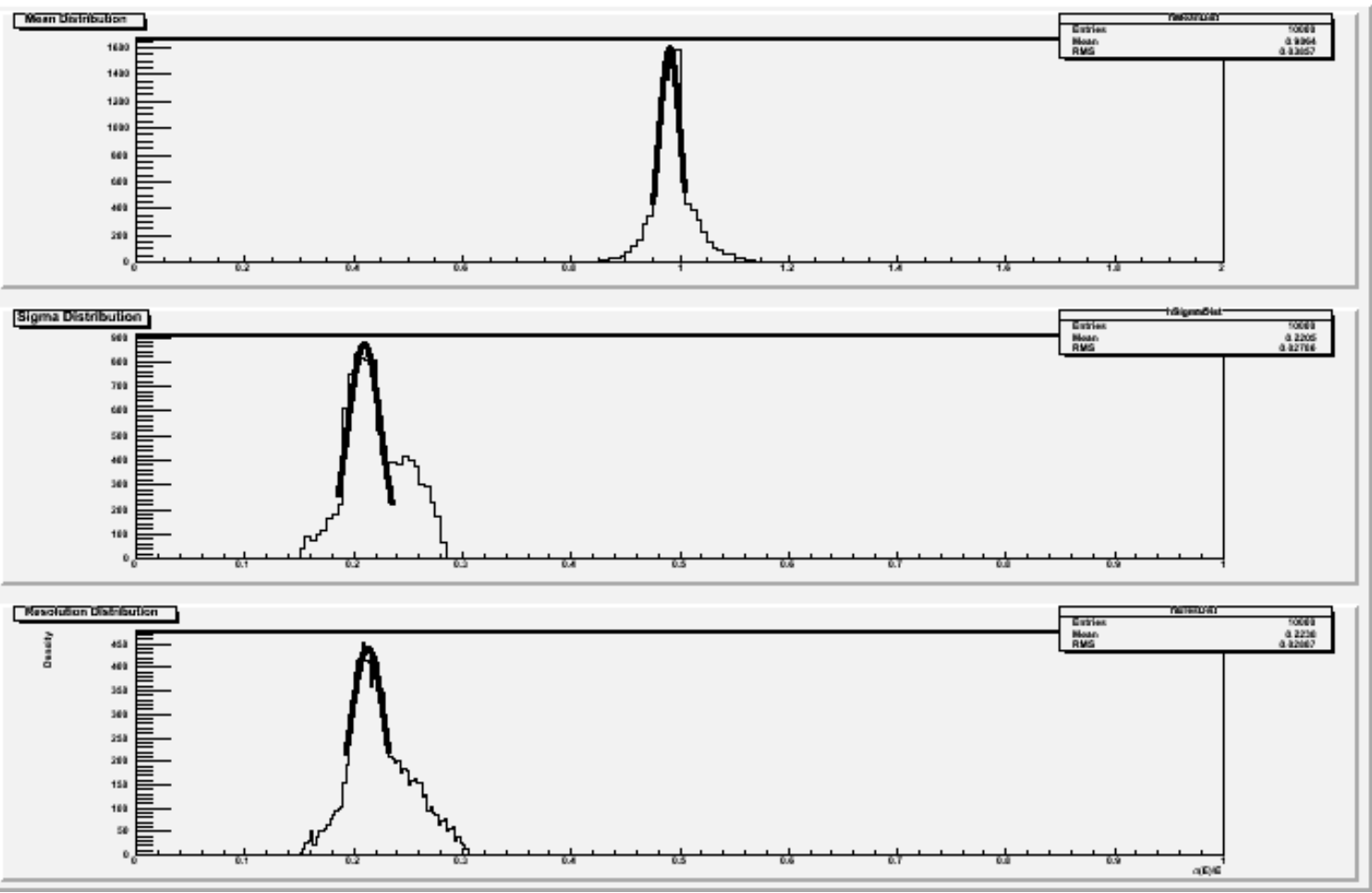

Figure D.4: Toy Monte Carlo distributions of fit parameters for the ratio EvtEnergyCorr/McEnergy, with $133.7 \mathrm{MeV}<$ McEnergy $<147.8 \mathrm{MeV}$. The top panel is the distribution of the mean, the middle panel is the distribution of sigma, and the bottom panel is the resolution distribution.

Subsequently, fill histograms for mean with $\langle E\rangle$ and resolution plot with $\sigma(E) /<$ $E>$ for that trial. This is done iterating over 10,000 trials. A depiction of the toy Monte Carlo procedure is shown in Figure D.4.

\section{D.5 Conclusions}

The ratio EvtEnergyCorr/McEnergy was be fitted as a double Gaussian, so trend of mean of the ratio $\langle E\rangle$ and $\sigma(E) /\langle E\rangle$ may be calculated. Likewise, a similar procedure was done for the ratio CTBBestEnergy/McEnergy. Differences as large as a few percent between the P6_V3 and P7_V2 MC photon samples values of the ratio EvtEnergyCorr/McEnergy were noted. It was observed that CTBBestEnergy is closer to McEnergy in P7_V2 than P6_V3: this represents an improvement in the use of this 
IRF. The P7_V2 energy resolution, as calculated from EvtEnergyCorr/McEnergy, appears to be slightly worse than P6V3. The fitting function described seems to provide an adequate fit in all cases. 


\section{References}

[1] S. I. Newton, Philosophical Writings. Cambridge Universty Press, 2004. 1

[2] S. T. Thornton and J. B. Marion, Classical Dynamics of Particles and Systems. Harcourt, 1995. 2

[3] E. Adelberger, B. Heckel, and A. Nelson Ann. Rev. Nucl. Part. Sci. 53 (2003) 77. 3

[4] A. Zee, Quantum Field Theory in a Nutshell. Princeton University Press, 2003. 3

[5] "http://www.clab.edc.uoc.gr/materials/pc/img/running_coupling1.gif," . 4

[6] N. Arkani-Hamed, S. Dimopoulos, and G. Dvali, "The hierarchy problem and new dimensions at a millimeter," Phys. Lett. B 429 (1998) 263, hep-ph/9803315, 4, 9]

[7] C. Hanhart, D. R. Phillips, S. Reddy, and M. J. Savage, "Extra dimensions, sn1987a, and nucleon-nucleon scattering data," Nucl. Phys. B 595 335, nucl-th/0007016, 6, 33, 35, 36, 37,

[8] J. Phys. G: Nucl. and Part. Phys. 37 (2010) 75021, 6, 79

[9] T. G. Rizzo, "Pedagogical Introduction to Extra Dimensions," in $S L A C$ Summer Institute on Particle Physics (SSI2004). 2004. 17, 101]

[10] N. Arkani-Hamed, S. Dimopoulos, and G. R. Dvali, "Phenomenology, astrophysics and cosmology of theories with sub-millimeter dimensions and tev 
scale quantum gravity," Phys. Rev. D59 (1999) 086004, hep-ph/9807344. 9, 102

[11] S. Cullen and M. Perelstein, "Sn1987a constraints on large compact dimensions," Phys. Rev. Lett. 83 (1999) 268. hep-ph/9903422, 9

[12] C. Hoyle, D. Kapner, B. Heckel, E. Adelberger, J. Gundlach, U. Schmidt, and H. Swanson, "Submillimeter tests of the gravitational inverse square law," Phys. Rev. D 70 (2004) 042004. 10]

[13] D. Kapner et al., "Tests of the gravitational inverse-square law below the dark-energy length scale," Phys. Rev. Lett. 98 (January, 2007) 021101. 10]

[14] A. A. Geraci, S. J. Smullin, D. M. Weld, J. Chiaverini, and A. Kapitulnik, "Improved constraints on non-newtonian forces at 10 microns," Phys. Rev. D 78 (2008) , 0802.2350v1. 10, 11]

[15] S. Murgia, Search for Large Extra Dimensions with Diphoton Events at CDF. PhD thesis, Michigan State University, 2002. 12

[16] C. Amsler et al. Phys. Lett. B667 (2008) 1. 12, 132

[17] R. Franceschini, P. P. Giardino, G. F. Giudice, P. Lodone, and A. Strumia, "Lhc bounds on large extra dimensions," JHEP 05 (2011) 092. arXiv:hep-ph/1101.4919, 14, 91

[18] S. Hannestad and G. G. Raffelt, "Stringent neutron-star limits on large extra dimensions," Phys. Rev. Lett. 88 (2002) 071301, arXiv:hep-ph/0110067, 13

[19] M. Cassé, J. Paul, G. Bertone, and G. Sigl, "Gamma rays from the galactic bulge and large extra dimensions," Phys. Rev. Lett. 92 no. 11, (Mar, 2004) 111102, 13, 106,

[20] L. Randall and R. Sundrum, "A large mass hierarchy from a small extra dimension," Phys. Rev. Lett. 83 (1999) 3370, hep-ph/9905221v1, 16]

[21] P. Fox JHEP 0102 (2001) 022, hep-ph/0106343v2, 16, 101, 
[22] D. Hooper and S. Profumo, "Dark Matter and Collider Phenomenology of Universal Extra Dimensions," Phys. Rep. 453 no. 2-4, (2007) 29 - 115. 17

[23] W. Atwood et al., "The Large Area Telescope on the Fermi Gamma-Ray Space Telescope Mission," ApJ 697 (June, 2009) 1071-1102, arXiv:0902.1089, 22. 28, 32

[24] Proceedings of the 31st ICRC. July, 2009. 28

[25] "FSSC: data data analysis online documentation science tools: Cicerone LAT IRFs." http://fermi.gsfc.nasa.gov/ssc/data/analysis/documentation/Cicerone/. 29

[26] "Pass6_v3 performance page for public use - GLAST LAT science groups SLAC confluence." http://confluence.slac.stanford.edu/x/34BLAw, 30

[27] D. J. Thompson et al. Astrophys. J. Suppl. 86 (1993) 629-656. 32

[28] S. Hannestad and G. G. Raffelt, "Supernova and Neutron-star Limits on Large Extra Dimensions Reexamined," Phys. Rev. D 67 no. 12, (Jun, 2003) 125008, hep-ph/0304029v2, 33, 36, 69, 91,

[29] G. G. Raffelt, Stars as Laboratories for Fundamental Physics. The University of Chicago Press, 1996. 35, 60, 103,

[30] P. Sreekumar et al., "EGRET Observations of the Extrgalactic Gamma-Ray Emission," Astrophys. J. 494 (1998) 523, 38, 105]

[31] S. Hannestad and G. Raffelt, "New supernova limit on large extra dimensions," Phys. Rev. Lett. 87 (2001) 051301, hep-ph/0103201v2, 38

[32] H. Goldstein, C. Poole, and J. Safko, Classical Mechanics. Addison Wesley, 3 ed., 2002. 43

[33] T. Erber, "High-energy electromagnetic conversion processes in intense magnetic fields," Rev. Mod. Phys. 38 (1966) 626. 49 
[34] V. Berestetskii, E. Lifshitz, and L. Pitaeveskii, Quantum Electrodynamics, vol. 4 of Landau and Lifshitz Course of Theoretical Physics. Pergamon Press, 2nd ed., 1982. 49

[35] S. Rosswog and M. Brüggen, Introduction to High Energy Astrophysics. Cambridge University Press, 2007. 60, 103

[36] R. J. Hall, 2006. http://en.wikipedia.org/wiki/File:Core_collapse_scenario.png. 61

[37] G. Woan, The Cambridge Handbook of Physics Formulas. Cambridge University Press, 2000. 64

[38] D. Backer Nature 228 (1970) 1297. 64

[39] R. Manchester, G. Hobbs, A. Teoh, and M. Hobbs, "The atnf pulsar catalogue," Astronomical Journal 129 (2005) 1993-2006, astro-ph/0412641. http://www.atnf.csiro.au/research/pulsar/psrcat, 64, 65, 69

[40] W. Joye and E. Mandel vol. 295 of Astronomical Data Analysis Software and Systems XII ASP Conference Series, p. 489. 2003. http://adsabs.harvard.edu/abs/2003ASPC . .295 . .489J, 65, 73

[41] F. M. Walter, S. J. Wolk, and R. Neuhauser, "Discovery of a nearby isolated neutron star," Nature 379 (1996) 233-235. 66

[42] F. Walter Astrophys. J. 549 433. 66

[43] F. Walter and J. Lattimer Astrophys. J. 576 (2002) 145. 66

[44] M. H. van Kerkwijk and D. Kaplan, "Isolated neutron stars: Magnetic fields, distances, and spectra," Astrophys. Space Sci. 308 (2007) 191-201, astro-ph/0607320, 66

[45] M. H. van Kerkwijk and D. Kaplan, "Timing the Nearby Isolated Neutron Star RXJ1856.5-3754," Astrophys. J. 673 (2008) 163-166. 66 
[46] W. Becker, ed., Neutron Stars and Pulsars. Astrophysics and Space Science Library. Springer-Verlag, 2008. 66, 69]

[47] J. M. Lattimer, "Neutron Stars," in SLAC Summer Institute on Particle Physics (SSI2005). http://www.slac.stanford.edu/econf/C0507252/papers/L007.PDF, 66

[48] http://arecibo.tc.cornell.edu/PALFA/images/pulsar_model.jpg. 67

[49] T. Tauris, L. Nicastro, S. Johnston, R. Manchester, M. Bailes, A. Lyne, J. Glowacki, D. Lorimer, and N. D’Amico Astrophys. J. 428 (1994) L53. 68

[50] R. Mignani, G. Pavlov, and O. Kargaltsev Astron. \& Astrophys. 488 (2008) 1027. 68

[51] G. Pavlov, O. Kargaltsev, J. Wong, and G. Garmire Astrophysical Journal 691 (2009) 458. 68

[52] A. Deller, S. Tingay, M. Bailes, and J. Reynolds, "Precision southern hemisphere vlbi pulsar astrometry ii: Measurement of seven parallaxes," Astrophysical Journal 701 (2009) 1243-1257, arXiv:0906.3897v1[astro-ph.SR], 68

[53] J. M. Cordes and T. J. W. Lazio, "NE2001.I. A New Model for the Galactic Distribution of Free Electrons and its Fluctuations," ArXiv Astrophysics e-prints (July, 2002), arXiv:astro-ph/0207156, 68

[54] I. S. Shklovsky Astrophys. Lett. 8 (1970) 101. 68

[55] W. Becker, A. Jessner, M. Kramer, V. Testa, and C. Howaldt Astrophys. J. 633 (2005) 367-376. 68, 69

[56] P. McCullough, B. D. Fields, and V. Pavlidou, "Discovery of an old, nearby, and overlooked supernova remnant centered on the constellation antlia pneumatica," Astrophys. J. 576 (2002) L41-L44. 69 
[57] C. Gwinn, J. Taylor, J. Weisberg, and L. Rawley, "Measurement of pulsar parallaxes by vlbi," Astron. J. 91 (1986) 338-342. 69, 70,

[58] W. F. Brisken, J. M. Benson, and W. Goss, "Very long baseline array measurement of nine pulsar parallaxes," Astrophys. J. 571 (2002) 906-917. 69

[59] J. Herfindal and J. Rankin, "Periodic nulls in the pulsar B1133+16," Mon. Not. R. Astron. Soc. 300 (2007) 430-436. 69

[60] R. Hoogerwerf, J. de Bruijne, and P. de Zeeuw, "On the origin of o and b-type stars with high velocities ii," Astron. E Astrophys. 367 (2001) 111-147, astro-ph/0010057v1, 70]

[61] "A brief introduction to FITS." http://fits.gsfc.nasa.gov/fits_intro.html, 71

[62] Gorski et al. Astrophys. J. 622 (2005) 759. http://healpix.jpl.nasa.gov/healpixSoftwareDocumentation.shtml. 73

[63] W. Cash Astrophys. J. 228 (1979) 939. 76

[64] J. Mattox et al., "The likelihood analysis of egret data," Astrophys. J. 461 (1996) 396. 76]

[65] F. James and M. Winkler, "MINUIT User's Guide," June, 2004. http://seal.web.cern.ch/seal/snapshot/work-packages/mathlibs/minuit/. 78

[66] J. Chiang. Slides, Fermi-LAT 2010 Collaboration Meeting, 2010. [79]

[67] A. Strong, I. Moskalenko, and O. Reimer Astrophys. J. 613 962-976. 82

[68] A. Strong Astrophys. Space Sci. 309 (2007) 35. 82

[69] 2004. http://lepexotica.web.cern.ch/LEPEXOTICA, 91 
REFERENCES

[70] V. Abazov et al., "Search for large extra spatial dimensions in the dielectron and diphoton channels in collisions," Phys. Rev. Lett. 102 (2009) 051601, 0809.2813v1. 91

[71] 16th International Workshop on Deep Inelastic Scattering and Related Subjects, Searches for Large Extra Dimensions at the Tevatron. July, 2008. arXiv:0807.0645v1, 91

[72] https://confluence.slac.stanford.edu/x/UxynBQ. 99]

[73] M. Giannoti, L. Duffy, and R. Nita JCAP 015 (2011) . 102

[74] Harding et al. Astrophysical Journal 476 (1997) 246. 105

[75] A. A. Abdo et al., "The Spectrum of the Isotropic Diffuse Gamma-Ray Emission Derived from First-Year Fermi Large Area Telescope Data," Phys. Rev. Lett. 104 (2010), astro-ph/1002.3603v1. 105

[76] M. Fairbairn Phys. Lett. B 508 (2001) 335-339, hep-ph/0101131, 107

[77] S. Hannestad Phys. Rev. D 64 (2001) 023515. 107

[78] A. A. Abdo et al., "Fermi large area telescope first source catalog," 188 no. 2, (2010) 405. http://stacks.iop.org/0067-0049/188/i=2/a=405, 125

[79] "Lat 1-year point source catalog." http://fermi.gsfc.nasa.gov/ssc/data/access/lat/1yr_catalog/, 126

[80] S. Agostinelli, J. Allison, K. Amako, J. Apostolakis, H. Araujo, P. Arce, M. Asai, D. Axen, S. Banerjee, G. Barrand, F. Behner, and L. Bellagamba, "Geant4 - a simulation toolkit," Nucl. Instrum. Methods A 506 (2003) 250-303. 129

[81] "Proceedings of a workshop held in perugia, italy, may 21st, 2003, and of a meeting on the analysis of data from astrophysics experiments (glast in particular)," in Science with the new generation of high energy gamma-ray 
experiments : between astrophysics and astroparticle physics, S. Ciprini, A. D. Angelis, P. Lubrano, and O. Mansutti, eds., p. 141. May, 2003. 129 